\title{
Improving Area Coverage by Mobile Sensor Networks
}

\author{
By \\ Xu Li \\ A Thesis submitted to the \\ Faculty of Graduate Studies and Research \\ In partial fulfillment of \\ The requirements for the degree of \\ Doctor of Philosophy \\ in \\ Computer Science
}

\author{
Ottawa-Carleton Institution for Computer Science \\ School of Computer Science \\ Carleton University \\ Ottawa, Ontario, Canada
}

Copyright (C) $2008 \mathrm{Xu} \mathrm{Li}$ 


$\begin{array}{ll}\begin{array}{l}\text { Library and } \\ \text { Archives Canada }\end{array} & \begin{array}{l}\text { Bibliothèque et } \\ \text { Archives Canada }\end{array} \\ \begin{array}{l}\text { Published Heritage } \\ \text { Branch }\end{array} & \begin{array}{l}\text { Direction du } \\ \text { Patrimoine de l'édition }\end{array} \\ \begin{array}{l}\text { 395 Wellington Street } \\ \text { Ottawa ON K1A 0N4 } \\ \text { Canada }\end{array} & \begin{array}{l}\text { 395, rue Wellington } \\ \text { Ottawa ON K1A 0N4 } \\ \text { Canada }\end{array}\end{array}$

Your file Votre référence ISBN: 978-0-494-47481-5 Our file Notre référence ISBN: 978-0-494-47481-5

NOTICE:

The author has granted a nonexclusive license allowing Library and Archives Canada to reproduce, publish, archive, preserve, conserve, communicate to the public by telecommunication or on the Internet, loan, distribute and sell theses worldwide, for commercial or noncommercial purposes, in microform, paper, electronic and/or any other formats.

The author retains copyright ownership and moral rights in this thesis. Neither the thesis nor substantial extracts from it may be printed or otherwise reproduced without the author's permission.
AVIS:

L'auteur a accordé une licence non exclusive permettant à la Bibliothèque et Archives Canada de reproduire, publier, archiver, sauvegarder, conserver, transmettre au public par télécommunication ou par l'Internet, prêter, distribuer et vendre des thèses partout dans le monde, à des fins commerciales ou autres, sur support microforme, papier, électronique et/ou autres formats.

L'auteur conserve la propriété du droit d'auteur et des droits moraux qui protège cette thèse. $\mathrm{Ni}$ la thèse ni des extraits substantiels de celle-ci ne doivent être imprimés ou autrement reproduits sans son autorisation.
In compliance with the Canadian Privacy Act some supporting forms may have been removed from this thesis.

While these forms may be included in the document page count, their removal does not represent any loss of content from the thesis.
Conformément à la loi canadienne sur la protection de la vie privée, quelques formulaires secondaires ont été enlevés de cette thèse.

Bien que ces formulaires aient inclus dans la pagination, il n'y aura aucun contenu manquant.

\section{Canada}




\title{
Improving Area Coverage by Mobile Sensor Networks
}

\author{
By \\ Xu Li

\begin{abstract}
A Thesis submitted to the
Faculty of Graduate Studies and Research
\end{abstract} \\ In partial fulfillment of \\ The requirements for the degree of \\ Doctor of Philosophy \\ in \\ Computer Science
}

\author{
Ottawa-Carleton Institution for Computer Science \\ School of Computer Science \\ Carleton University \\ Ottawa, Ontario, Canada
}

Copyright (C) $2008 \mathrm{Xu} \mathrm{Li}$ 


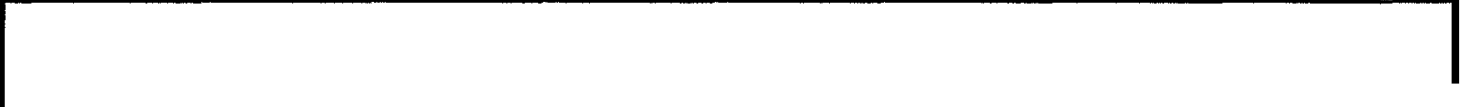

ABSTRACT

n mobile sensor networks, node mobility can be wisely used to improve coverage. There
are two approaches, sensor self-deployment and sensor relocation, which aim respectively at achieving maximized hole-free coverage from a randomized node dropping and at maintaining constant coverage in face of node failure. In this thesis, we study in depth existing sensor self-deployment algorithms and sensor relocation protocols. We pinpoint a new problem of focused coverage formation around a Point of Interest (POI) and formalize an emerging distance-sensitive service discovery problem.

For the first problem, we devise two solutions, GA and GRG, which are the first localized sensor self-deployment algorithms that provide coverage guarantee. They drive sensors to move along a triangle tessellation to surround the POI. In GA, sensors greedily proceed as close to the POI as they can; in GRG, when their greedy advance is blocked, they rotate around the POI to a vertex where greedy advance can resume. For the second problem, we devise a localized solution iMesh, where service providers construct a novel planar structure, information mesh, with good proximity property; service consumers conduct a lookup process restricted within their home mesh cells to discover nearby services.

Last but not least, we also develop two localized sensor relocation protocols, ZONER and MSRP, both of which have significant advantages over previous similar algorithms. 


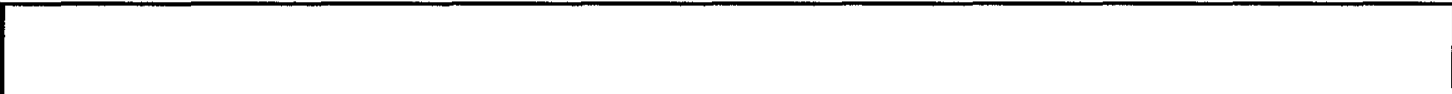
ACKNOWLEDGMENTS

I would like to thank my thesis supervisors, Dr. Nicola Santoro and Dr. Ivan Stojmenovic, for their precious scientific direction and financial support on this work. Without their supervision, I could not have finished it. I would also like to show special gratitude to Dr. Hannes Frey for his collaboration in developing the sensor self-deployment algorithms GA and GRG presented in Chapter 3. Finally, I want to thank my parents and my wife Kaiyuan Lu for their unconditional support and constant encouragement.

This work was partially supported by NSERC Strategic and Doctoral grants and UK Royal Society Research Merit Award. 
List of Tables $\quad$ xi

1 Introduction 1

1.1 Sensor networks in a nutshell ................... 2

1.1.1 Wireless sensor networks (WSNs) ............. 2

1.1.2 Mobile sensor networks (MSNs) . . . . . . . . . . . 3

1.1.3 Sensing models and area coverage ............ 5

1.2 Improve coverage in MSN $\ldots \ldots \ldots \ldots$

1.2.1 Two streams of solution algorithms ............ 7

1.2.2 A general algorithm classification .............. 11

1.2.3 Design considerations . . . . . . . . . . . . . . 12

1.3 Motivation . . . . . . . . . . . . . . . . . . . 13

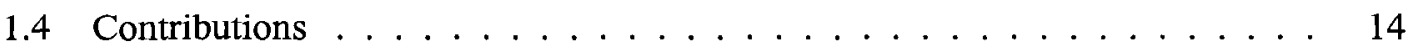

1.4 .1 Literature review . . . . . . . . . . . . . . . 14

1.4.2 Sensor self-deployment . . . . . . . . . . . . 15

1.4 .3 Service discovery . . . . . . . . . . . . . . 16 
1.4 .4 Sensor relocation . . . . . . . . . . . . . . . 18

2 Literature Review 19

2.1 Sensor self-deployment algorithms . . . . . . . . . . . . . . . 20

2.1 .1 Vector-based approach .................. 20

2.1 .2 Incremental approach . . . . . . . . . . . . 27

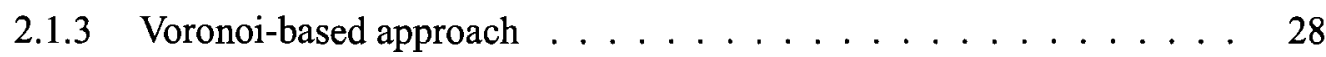

2.1 .4 Load-balancing approach . . . . . . . . . . . . . . . . 29

2.1.5 Maximum-flow approach . . . . . . . . . . . . 31

2.1 .6 Stochastic approach $\ldots \ldots \ldots 33$

2.1 .7 Point-coverage approach $\ldots \ldots \ldots \ldots$. . . . . . . . . 34

2.1.8 Genetic Algorithm approach . . . . . . . . . . . . . 35

2.2 Sensor relocation protocols $\ldots \ldots \ldots \ldots \ldots \ldots$

2.2.1 Broadcast-based approach . . . . . . . . . . . 37

2.2.2 Quorum-based approach .................... 39

2.3 Other previous related work $\ldots \ldots \ldots \ldots \ldots \ldots$

2.3.1 Greedy-Face-Greedy routing . . . . . . . . . . . . 41

2.3.2 Location services and data centric storage schemes . . . . . . . . 44

3 Focused Coverage by Mobile Sensors I

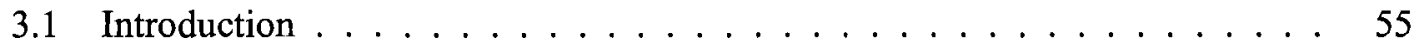

3.1 .1 Our contributions . . . . . . . . . . .... 56

3.2 Model and Definitions $\ldots \ldots \ldots \ldots \ldots$

3.3 Equilateral Triangle Tessellation . . . . . . . . . . . . . . . . 59

3.4 Protocol Description $\ldots \ldots \ldots \ldots$. . . . . . . . . . . . 61

3.4 .1 Greedy Advance $(\mathrm{GA}) \ldots \ldots \ldots 61$

3.4 .2 Greedy-Rotation-Greedy $(\mathrm{GRG}) \ldots \ldots \ldots 3$

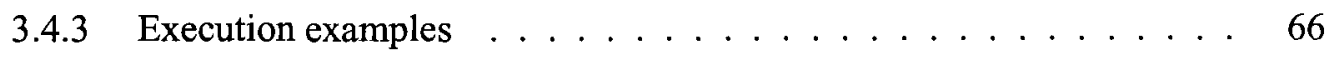


3.4 .4 Resolving node collision . . . . . . . . . . . . . . 68

3.5 Analysis ............................. 70

3.5.1 Correctness . . . . . . . . . . . . . . . 70

3.5 .2 Coverage radius . . . . . . . . . . . . . . . 73

3.6 Performance Evaluation . . . . . . . . . . . . . . . . . . . 77

3.6.1 Evaluation metrics . . . . . . . . . . . . . . 77

3.6 .2 Simulation setup $\ldots \ldots \ldots . \ldots . \ldots . \ldots 79$

3.6 .3 Experimental results $\ldots \ldots \ldots 7 . \ldots \ldots$

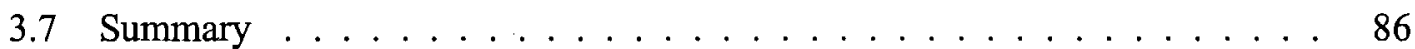

4 Focused Coverage by Mobile Sensors II $\quad 87$

4.1 Introduction . . . . . . . . . . . . . . . 88

4.1 .1 Our contributions . . . . . . . . . . . . . . 88

4.2 Model and Definitions $\ldots \ldots \ldots \ldots$

4.3 Deployment polygon . . . . . . . . . . . . . . . . . . . . 89

4.3.1 Definition .......................... 90

4.3 .2 Properties . . . . . . . . . . . . . . . . . 91

4.4 Protocol Description $\ldots \ldots \ldots$. . . . . . . . . . . . . . . 94

4.4 .1 Greedy ............................... 95

4.4.2 Rotation . . . . . . . . . . . . . . . . . 96

4.4 .3 Collision avoidance . . . . . . . . . . . . . . . 97

4.5 Performance evaluation . . . . . . . . . . . . . . . 100

4.5.1 Simulation setup $\ldots \ldots \ldots 101$

4.5.2 Experimental results . . . . . . . . . . . . . 102

4.6 Summary . . . . . . . . . . . . . . . . . . . . 104

5 Distance-Sensitive Service Discovery 106

5.1 Introduction . . . . . . . . . . . . . . . 107 
5.1 .1 Our contributions . . . . . . . . . . . . . . . . . 109

5.2 Model and definitions . . . . . . . . . . . . . . . . . . 110

5.3 Protocol description . . . . . . . . . . . . . . . . . . 111

5.3 .1 Basic iMesh . . . . . . . . . . . . . . . 111

5.3 .2 Complete iMesh ..................... 121

5.4 Implementation details $\ldots \ldots \ldots \ldots \ldots \ldots$

5.4.1 Information mesh construction . . . . . . . . . . . . . . . 123

5.4 .2 Distance-sensitive service lookup . . . . . . . . . . . . . 125

5.4.3 Tolerating node failures . . . . . . . . . . . . . 126

5.4.4 Keeping storage load constant . . . . . . . . . . . . . . . . 127

5.5 Performance Evaluation . . . . . . . . . . . . . . . . . 127

5.5 .1 Evaluation metrics . . . . . . . . . . . . . . 128

5.5 .2 Simulation setup $\ldots \ldots \ldots \ldots \ldots$

5.5 .3 Experimental results . . . . . . . . . . . . . . . . . 129

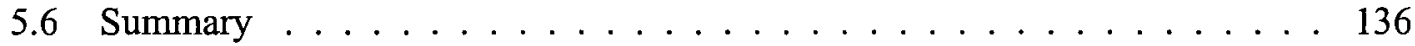

6 Autonomous Sensor Relocation $\quad 137$

6.1 Introduction . . . . . . . . . . . . . . . . . . . 138

6.1 .1 Our contributions . . . . . . . . . . . . . . . 139

6.2 Model and definitions . . . . . . . . . . . . . . . . . . . . 141

6.3 Range-restricted geographic flooding . . . . . . . . . . . . . . . . . 142

6.4 ZONER: a ZONE-based sensor Relocation protocol . . . . . . . . . . . . . . 144

6.5 MSRP: a Mesh-based Sensor Relocation Protocol . . . . . . . . . . . . . . . 146

6.6 Fault Tolerance . . . . . . . . . . . . . . . . . . . . 148

6.7 Contention Resolution $\ldots \ldots \ldots$

6.8 Protocol Analysis . . . . . . . . . . . . . . . . . . . 150

6.9 Summary . . . . . . . . . . . . . . . . . . 153 
7 Conclusions $\quad 155$

7.1 Sensor self-deployment . . . . . . . . . . . . . . . . . 155

7.1.1 Open Problems . . . . . . . . . . . . . . . . . . . 156

7.2 Service discovery . . . . . . . . . . . . . . 158

$7.2 .1 \quad$ Open problems $\ldots \ldots \ldots \ldots \ldots \ldots$

7.3 Sensor relocation $\ldots \ldots \ldots \ldots \ldots$

7.3 .1 Open problems $\ldots \ldots \ldots \ldots 1 \ldots \ldots \ldots \ldots$

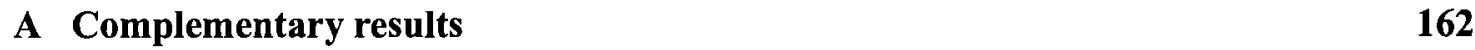

A.1 Intersection transformation . . . . . . . . . . . . . . 162

A.2 iMesh in a WSN of 1,000 nodes $\ldots \ldots \ldots \ldots 3$

$\begin{array}{ll}\text { B Acronyms } & 165\end{array}$

$\begin{array}{ll}\text { References } & 168\end{array}$ 


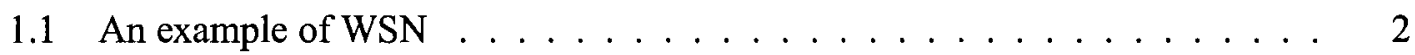

1.2 Mobile sensors ...................... 4

1.3 Two different sensing models . . . . . . . . . . . . . . 5

1.4 Shifted migration $\ldots \ldots \ldots \ldots \ldots$

2.1 An example of movement vector . . . . . . . . . . . . . . 21

2.2 An example of Voronoi polygon . . . . . . . . . . . . . . 28

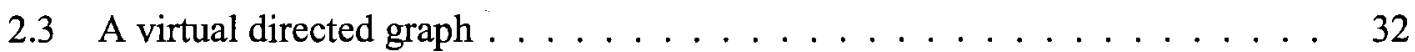

2.4 MR-based stochastic movement $\ldots \ldots \ldots \ldots$

2.5 Crossover operations over a single chromosome . . . . . . . . . . . 36

2.6 Face routing in $\mathrm{GFG} \ldots \ldots \ldots \ldots$

2.7 An example of the quorum-based location service . . . . . . . . . . 45

2.8 An example of the grid location service $\ldots \ldots \ldots$. . . . . . . . . . 47

2.9 An illustration of GHT . . . . . . . . . . . . . . . . . . . 48

2.10 An illustration of Landmark . . . . . . . . . . . . . . . . . . 51

2.11 Distance-sensitive retrieval $\ldots \ldots \ldots \ldots \ldots$

3.1 Circular coverage radius $\ldots \ldots \ldots \ldots \ldots$

3.2 Polygonal (hexagonal) coverage radius . . . . . . . . . . . . 56 
3.3 An partial equilateral triangle tessellation $\ldots \ldots \ldots 60$

3.4 Hop selection in $\mathrm{GA} \ldots \ldots \ldots \ldots \ldots$

3.5 Hop selection in GRG . . . . . . . . . . . . . . . . . . . 63

3.6 Final node distribution after sensor self-deployment . . . . . . . . . . . 67

3.7 An example of collision loop in GRG-CW . . . . . . . . . . . . . . . . 69

3.8 Redundancy . . . . . . . . . . . . . . . . . . . . . . 75

3.9 Results from a network of size varying from $v(1)$ to $v(10)$ and a dropping area of size fixed to $200^{2} \ldots \ldots \ldots \ldots \ldots$

3.10 Results from a network of size fixed to $v(7)$ and a dropping area of size varying

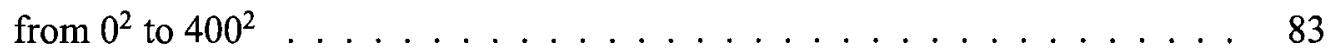

4.1 Relation diagram $\ldots \ldots \ldots \ldots$. . . . . . . . . . . . . 90

4.2 Deployment polygons $\mathcal{P}_{14}$ and $\mathcal{P}_{15} \ldots \ldots \ldots \ldots \ldots$

4.3 Pictures for proving Lemmas 4.2 and $4.3 \ldots \ldots \ldots$. . . . . . . . . 92

4.4 Neighborhood patterns of vertex $v \ldots \ldots \ldots$. . . . . . . . . 93

4.5 The leftmost inward vertex neighbor $a$ of $v \ldots \ldots \ldots$. . . . . . 98

4.6 Number of nodes $(\mathrm{nN})$ v.s. Coverage radius $(\mathrm{cR}) \ldots \ldots \ldots 1$

4.7 Experimental results $\ldots \ldots \ldots \ldots$. . . . . . . . . . . . . . . . . .

5.1 Information mesh construction $\ldots \ldots \ldots \ldots \ldots \ldots \ldots \ldots \ldots$

5.2 Cross lookup . . . . . . . . . . . . . . . . . . . 114

5.3 Blocking chain $p_{0} \stackrel{6}{\leftarrow} p_{6}\left(p_{0} \stackrel{u_{0}}{\leftarrow} p_{1} \stackrel{u_{1}}{\leftarrow} \ldots \stackrel{p_{5}}{\leftarrow} p_{6}\right) \ldots \ldots \ldots 115$

5.4 An information mesh of $\eta=O(v+\sqrt{n}) \ldots \ldots \ldots 117$

5.5 Example situations of $1<T C R(a) \leq 2 \ldots \ldots \ldots \ldots$

5.6 Example situations of $T C R(a)>2 \ldots \ldots \ldots \ldots \ldots \ldots$

5.7 An amplified version of the circled part in Figure 5.5(b) . . . . . . . . . 120

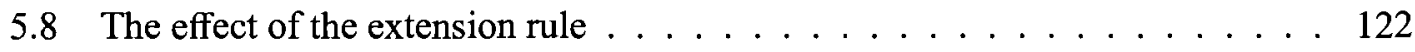

5.9 Information mesh construction in an arbitrary sensor network . . . . . . . . . 124 
5.10 Cross lookup in an arbitrary sensor network . . . . . . . . . . . 125

5.11 Perimeter lookup in an arbitrary sensor network . . . . . . . . . . 126

5.12 Message overhead in a WSN of 10,000 nodes $\ldots \ldots \ldots$. . . . . . . . . 129

5.13 Distance sensitivity in a network of 10,000 nodes . . . . . . . . . . . 131

5.14 iMesh v.s. Quorum in a WSN of 10,000 nodes $\ldots \ldots 133$

5.15 iMesh v.s. Quorum in a WSN of 1,000 nodes $\ldots \ldots \ldots 133$

5.16 Proportional Difference of Construction Cost (PDOCC) of iMesh and Quorum 135

6.1 A general view of how ZONER works . . . . . . . . . . . . . . 140

6.2 A general view of how MSRP works . . . . . . . . . . . . . . . 141

6.3 An illustration of closed traversal circles . . . . . . . . . . . . . . . . 143

7.1 A 3-tier information mesh . . . . . . . . . . . . . 159

A.1 A pair of crossover links $a b$ and $c d \ldots \ldots \ldots 2$

A.2 TCR in a WSN of 1,000 nodes $\ldots \ldots \ldots \ldots$

A.3 PTCR in a WSN of 1,000 nodes $\ldots \ldots \ldots \ldots$. . . . . . . . . 164 


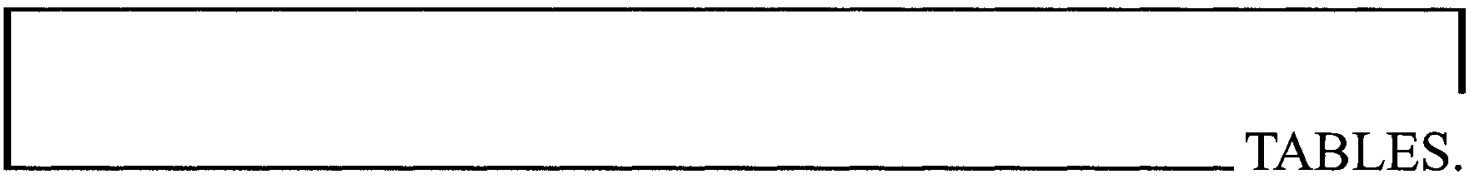

2.1 Algorithm comparison . . . . . . . . . . . . . . . . 20

3.1 Notations . . . . . . . . . . . . . . . . . . 58

4.1 Notations . . . . . . . . . . . . . . . . . . . . . . . 89

4.2 Neighborhood patterns . . . . . . . . . . . . . . . . . . . . . . 94

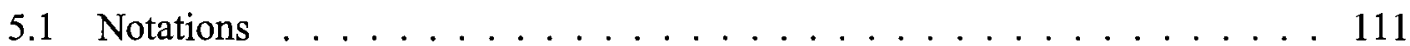

6.1 Protocol comparison . . . . . . . . . . . . . . . . 150 


\section{CHAPTER 1}

INTRODUCTION

I

$\mathrm{n}$ recent years, a new paradigm of sensor networks, mobile sensor networks (MSNs), are

emerging. They inherit all the properties from static wireless sensor networks (WSNs) and meanwhile have their own uniqueness - node mobility. This powerful locomotion capability enables sensors to act in a much intelligent way, e.g., escape from dangerous situations or physically respond to important events, and renders MSNs more flexible and adaptive to unknown and/or hazardous environment than WSNs. An increasing amount of research attention is currently drawn to MSNs.

Throughout the thesis, we use the following conventional notations: $r_{s}$ represents a sensor's sensing radius; $r_{c}$ stands for a sensor's communication radius. In this introductory chapter, we first give a general view of sensor networks in Section 1.1.1 and then introduce the focus of the thesis, i.e., improving area coverage by MSNs, in Section 1.2. Thereafter, we present the motivation of this thesis in Section 1.3 and summarize its contributions in Section 1.4 . 


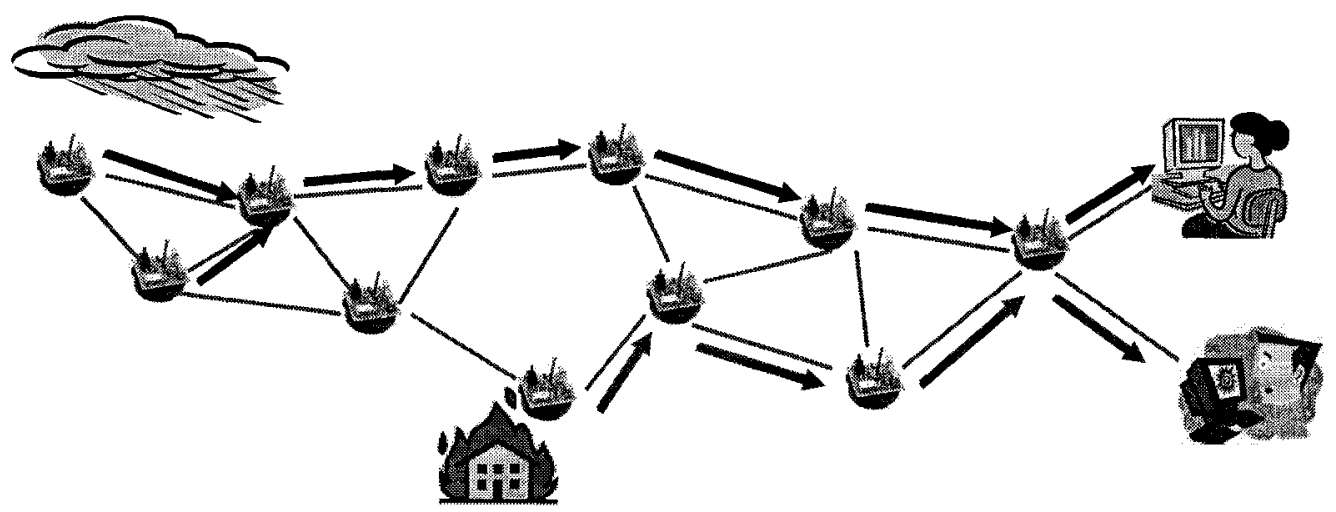

figure credit: Internet resource

Figure 1.1: An example of WSN

\subsection{Sensor networks in a nutshell}

\subsubsection{Wireless sensor networks (WSNs)}

Wireless sensors are micro-sized sensing devices that are equipped with radio transceivers and powered by low-energy batteries. They are able to respond to physical stimulus, e.g., temperature, light, vibration, humidity, etc., by producing radio signals and to communicate via radio frequency $(\mathrm{RF})$. Wireless sensor networks (WSNs) [7,53] are networks composed of such wireless sensors. They are often randomly deployed, e.g., by aircraft, for surveillance applications in unknown and/or hazardous environments, where physical dynamics and spatiotemporal irregularities prevail. In WSNs, sensors sample their surroundings and report through multi-hop communication paths to predefined data sinks, where raw sensor data are integrated to determine environmental changes or detect interesting events. WSNs are known as

- large-scale infrastructureless network: a WSN usually contains hundreds, or even thousands, of sensor nodes, which are densely scattered in the monitored area and share network control with each other because of the absence of centralized administration;

- resource-constrained network: for cheap and mass production, sensors are built with low-energy batteries, constrained computation resources (e.g., storage space and CPU 
cycle) and limited communication capabilities (e.g., short transmission range, low transmission rate and narrow bandwidth);

- unattended failure-prone network: a WSN is administrated without human intervention as manual configuration is usually impractical or infeasible due to operational factors, and it suffers from various unpredictable node failures for power exhaust, software errors, hardware defects, external attacks and harsh environmental conditions.

Figure 1.1 shows an application example of WSN, where thunder storm and residence fire are detected and reported to data sinks. As a bridge between human and the physical world [21], WSNs hold the promise of revolutionizing our daily life on the one hand. On the other hand, their realization confronts many challenges ranging from physical layer to application layer, e.g., localization [10], media access control [17], routing [26], data diffusion [19], topology control [66], time synchronization [71] and coverage [55], just to name a few. The difficulties are even augmented by the above three negative properties of WSNs. After almost a decade of investigation, many of these problems have been well understood; some others still remain in doubt, rendering the research on WSNs reach a semi-mature state [50].

\subsubsection{Mobile sensor networks (MSNs)}

Mobile sensor networks (MSNs) are a new paradigm of WSNs that emerged a few years ago. They inherit all the properties from static WSNs and meanwhile featured with their own particularity, node mobility. In MSNs, nodes are attached to certain mobile platforms and therefore obtain the ability to geographically relocate themselves as needed. Note that, unlike another mobility-enriched type of wireless networks, mobile ad hoc networks (MANETs) [12], where node mobility is a uncontrolled interferential factor, MSNs have it as a controllable asset, by which they can be tuned more intelligent and more flexible and adaptive to their monitored environments than static WSNs. In MSNs, there are two mobility models: 


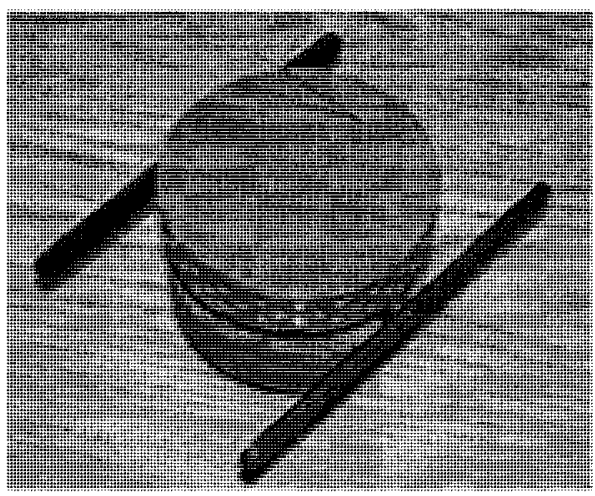

(a) A hopping sensor (IMLM unit [1])

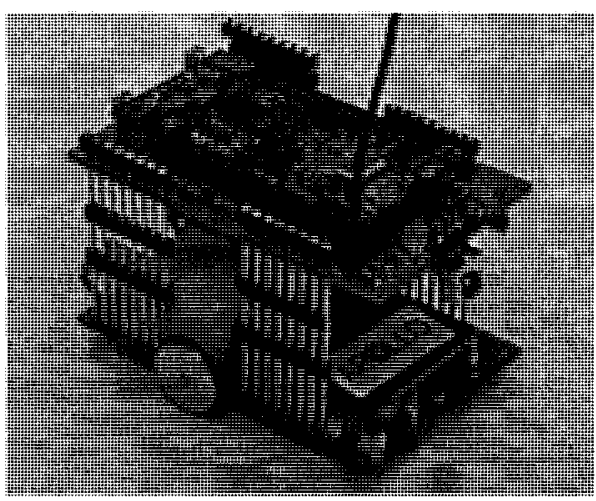

(b) A running sensor (Robomot [20])

Figure 1.2: Mobile sensors

- hopping model: Sensors are driven by propellers such as piston [1] or coiled spring [18]. They are able to hop step by step, each step up to a certain maximum distance. Propeller dynamics and natural conditions (e.g., wind and heavy rain) may affect their hopping performance, leading to errors in both direction and distance.

- running model: Sensors are mounted on mini mobile robots [14,20,54] that are able to move continuously and accurately to a designated location.

Figure 1.2 shows two example types of mobile sensors corresponding to the above two mobility models. Leveraged by controlled node mobility, many design challenges of WSNs mentioned in Section 1.1.1 are now encompassing a whole new set of possibilities and ideas. For example, by autonomous geographic relocation, mobile nodes may improve coverage to provide quality sensing service [33], build routes to facilitate date communication [49], harvest energy before their battery power is depleted [63], avoid and fix network partition to assure message transmission [70], achieve desired node density to avoid energy hole and increase network lifetime [85], and many more. Among these interesting research issues is the mobility-controlled coverage improvement problem constitutes the focus of this thesis. 


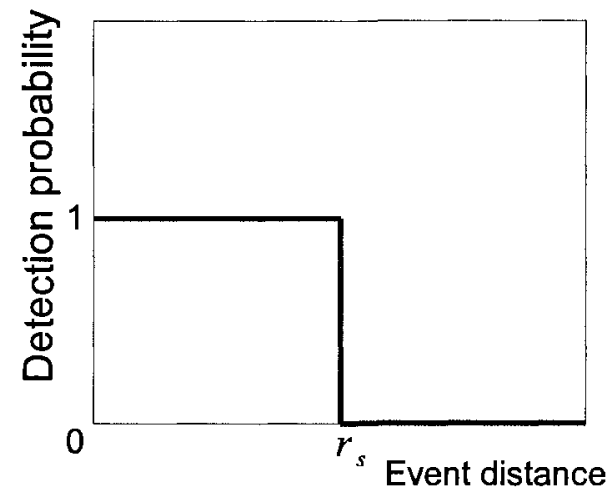

(a) The binary model

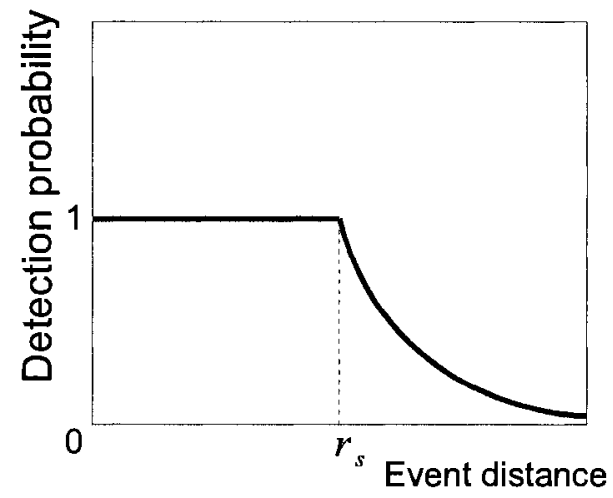

(b) A probabilistic model $[34,88]$

Figure 1.3: Two different sensing models

\subsubsection{Sensing models and area coverage}

Consider a sensor $i$ and a point $x$. Denote by $d_{E}(i, x)$ the Euclidean distance from $i$ to $x$ and by $p_{i}(x)$ the probability that $i$ detects a concerned event occurring at $x$. There are two different sensing models: binary model and probabilistic model. The former is an ideal one that has been widely adopted in the literature. By this model, sensor $i$ either detects or does not detect the event without uncertainty, depending on whether or not the event location $x$ is within its sensing range $r_{s}$ (the range of its sensing capability). Formally, it defines that

$$
p_{i}(x)= \begin{cases}1, & \text { for } \quad d_{E}(i, x) \leq r_{s} \\ 0, & \text { for } \quad d_{E}(i, x)>r_{s}\end{cases}
$$

The latter is a more realistic model where $p_{i}(x)$ follows certain probability distribution, reflecting the gradual degradation of physical signals in practice. The definition of $p_{i}(x)$ is subject to the material and the hardware technology that a sensor uses. For example, in $[34,88]$, it is

$$
p_{i}(x)=\left\{\begin{array}{lll}
1, & \text { for } & d_{E}(i, x) \leq r_{s} \\
e^{-\alpha\left(d_{E}(i, x)-r_{s}\right)}, & \text { for } & d_{E}(i, x)>r_{s}
\end{array}\right.
$$


where $\alpha$ is a factor that describes how fast the sensing capacity of $i$ fades with distance; this exponential model reflects the behavior of range sensing devices like infrared and ultrasound sensors in reality. Figure 1.3 illustrates the binary model and the exponential model. Some other probabilistic sensing models can be found in $[4,47]$.

Consider an area $\mathcal{A}$. In the binary model, it is said to be covered by $n$ sensors if it is within the $n$ sensors' sensing range; in the probabilistic model, it is said to be probabilistically covered by $n$ sensors with a given threshold value $\theta(0<\theta \leq 1)$ if $P(\mathcal{A})=1-\prod_{i=1}^{n}\left(1-p_{i}(x)\right) \geq \theta$ for every point $x$ in $\mathcal{A}$ [34]. The coverage region of a sensor network is the region enclosed by the outer boundary of the network. A sensing hole is a closed area, where no point is covered (probabilistically with threshold $\theta$ ) by any sensor, inside the coverage region. The area coverage (or simply coverage) of a sensor network is defined as the subtraction of the total area of sensing holes from the area of the coverage region. Size (i.e., area) and hole are the two key evaluation metrics for area coverage.

Lemma 1.1. [11,87] Under the binary sensing model, for a given number of nodes, an equilateral triangle tessellation node arrangement with separation $\sqrt{3} r_{s}$ produces maximized coverage with no sensing hole.

Lemma 1.2. [34] Under the exponential sensing model, for a given number of nodes, an equilateral triangle tessellation node arrangement with separation $\sqrt{3}\left(r_{s}-\frac{\ln (1-\sqrt[3]{1-\theta})}{\alpha}\right)$ produces maximized coverage with no sensing hole.

\subsection{Improve coverage in MSN}

The objective of a sensor network is to monitor its surroundings for event detection and object tracking $[7,53]$. Thus coverage is a functional basis of any sensor network. In order to well fulfill its assigned surveillance tasks, a sensor network should be deployed in the right region where concerned events may occur, and it should also cover the region of interest (ROI) continuously and maximally. In order to provide consistent sensing service without quality 
degradation, it should maintain a constant coverage over the ROI throughout its lifetime.

For static WSNs where there is no control on sensor placement, it is practically not possible to obtain and maintain a maximized hole-free coverage in face of stochastic node dropping and unpredictable node failure. Relevant research usually assume a large number of densely distrusted sensors and concentrates mainly on how to schedule sensor activities to save energy without jeopardizing existing coverage $[29,81]$. However, it may not be the case in MSNs where node mobility can be exploited to improve coverage, giving rise to the problem of mobility-controlled coverage improvement.

Mobility-controlled coverage improvement is a fundamental research problem in MSNs. It addresses how to achieve a maximized hole-free coverage out of a randomized node distribution and how to maintain existing coverage in presence of unpredictable node failures. There are two streams of solution approaches, i.e., sensor self-deployment and sensor relocation, which answer the two questions respectively.

We notice that there are algorithms that rely on a small number of mobile robots for mass node distribution [13] or individual node repairment [56] and emphasize on robot coordination. But these algorithms are not MSN-based coverage improvement algorithms. They in fact fall in a close but different research field, wireless sensor and actor networks (WSAN) [6], which is out of the scope of this thesis.

\subsubsection{Two streams of solution algorithms}

\section{Sensor self-deployment}

As senors are often dropped at random in an inaccessible terrain, we certainly can not assume that they have an optimal or desired placement at initiation. For instance, an emergency relief team use sensors to identify the leak source of noxious material in a reported factory; they compactly place sensors at the entrance without entering the factory so as not to risk being poisoned. Another example is that geologists airdrop sensors around the mouth of an active volcano to collect data like smoke, temperature change and earth vibration for eruption 
prediction; airdropping however causes a large degree of sensing range overlapping and even misplacement. In the cases of such compact or stochastic initial node distribution, sensors are expected to have the ability to self-deploy for hole elimination and coverage maximization.

Sensor self-deployment targets at the achievement of a desired overall node distribution without human assistance and often requires network-wide node reorganization. It is recognizedly related to the classic art gallery problem [60] in computation geometry and the robot exploration and mapping problem $[16,51,83,86]$ as well as the pattern formation problem $[25,69,76,82]$ in mobile robots. It was formally introduced by Howard, Mataric and Sukhatme $[37,38]$ in 2002 . Thereafter, it quickly grew to an important research subject and has been attracting an increasing amount of research attention. So far a number of sensor self-deployment algorithms have been proposed. Later, in Chapter 2, we will present a comprehensive survey of these algorithms.

From different perspectives, sensor self-deployment can be modeled as different problems and solved by different approaches. At the time of writing, there exist mainly eight different sensor self-deployment approaches as listed below:

- incremental approach [37], by which sensors are deployed incrementally, i.e., one at a time, base on the information gathered from previously deployed sensors;

- maximum-flow approach [18], by which sensors deployment is modeled as a minimum cost maximum flow problem from source regions to hole regions in a partitioned field;

- Genetic Algorithm approach [64], by which the final sensor movement plan is generated by multi-round plan selection and reproduction simulating genes and nature selection;

- Voronoi-based approach $[36,78]$, by which sensors adjust their location to reduce uncovered local area in its Voronoi polygon possibly in multiple rounds;

- load-balancing approach [84], by which the number of sensors in the regions of a partitioned sensor field is balanced through multiple rounds of scans; 
- vector-based approach $[33,36,38,52,62,78,88]$, by which sensors move according to a movement vector computed using the relative position of their neighbors;

- stochastic approach [59], by which sensors spread out through guided random walk;

- point-coverage approach [59], by which the area coverage problem is converted to a point coverage problem over certain geographic graph.

\section{Sensor relocation}

Sensor relocation concentrates on timely patching of each individual sensing hole without changing network topology. It can be used as a fault-tolerance technique to prevent coverage loss caused by node failures and thus keep coverage constant. Generally speaking, a sensor relocation protocol fulfills two tasks: replacement discovery, i.e., finding a pre-deployed/predetermined redundant sensor as the replacement of a failed one, and replacement migration, i.e., migrating the discovered replacement to the position of the failed sensor.

To minimize migration distance, the replacement should intuitively be a redundant node geographically closest to the failed node. In this case, replacement discovery is actually a distance-sensitive service discovery problem, which has not yet be addressed for WSNs/MSNs in the literature. Nevertheless, many generic service discovery algorithms have been proposed for wireless ad hoc networks. These algorithms can be categorized as directory-based approach and directory-less approach. The former, e.g. [39-41], use a well structured service directory to store service provider information and to facilitate service lookup. They usually require global computation such as clustering and dominating set formation for service directory construction and maintenance. The latter e.g. [24, 30,35], do not maintain any special component but rely on periodical service advertisement and multicasting-/anycasting- based service lookup. Their execution often involves (range-limited) flooding operations. These algorithms generate large message overhead and/or inconstant per node storage load and therefore are not suitable for WSNs/MSNs. A survey of service discovery algorithms in wireless ad hoc networks is beyond the scope of this thesis; yet it can be found in $[32,58]$. 


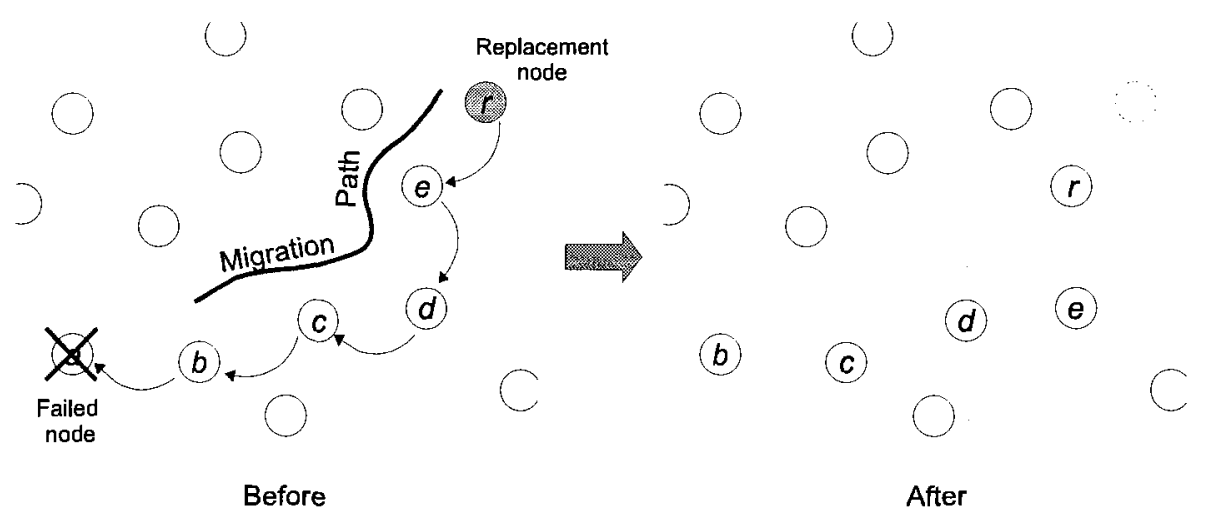

Figure 1.4: Shifted migration

Replacement migration can be accomplished either in a direct manner or in a shifted fashion. In direct migration, the replacement moves all the way to the position of the failed node. Due to the potentially long moving distance (which could be as bad as the spatial diameter of the network), this simple method may cause large migration delay and over-consume the battery power of the replacement node. In shifted migration, a multi-hop migration path is established between the replacement and the failed node, and every nodes along this path shifts its position towards the failed node, as shown in Figure 1.4. Compared with direct migration, this method may generate longer total moving distance and a larger number of moves, because the migration path is usually not shortest and often composed of multiple nodes. However, instead of punishing only one node, it distributes energy consumption for geographic migration among all the nodes along the path, prolonging network lifetime as a whole. In addition, it renders relocation latency subject to the longest hop (which is always less than the communication radius $r_{c}$ ) rather than the Euclidean distance between the replacement and the failed node. Under these circumstances, the shifted migration method is more desirable than the direct one, and the key of replacement migration then becomes energy-efficient migration path discovery, which is in fact a routing problem. Many good surveys of ad hoc routing protocols can be found in the literature $[5,8,26]$. 


\subsubsection{A general algorithm classification}

By the extent of its required/computed knowledge of the underlying network, a mobilitycontrolled coverage improvement scheme (i.e., a sensor self-deployment algorithm or a sensor relocation protocol) can be classified as centralized, distributed or localized.

\section{Centralized algorithm}

In a centralized scheme $[18,37,64]$, a pre-defined or elected central controller collects the information such as location and residual energy of all the nodes to build a global view of the network, and it outputs, based on this view, a motion plan for every node in the network. After its initial placement, each sensor node sends its information to, and then acquires and follows a motion plan from, the central controller.

Centralized algorithms have serious scalability problem and are also energy-consuming, especially in multi-hop networks like MSNs. It is because they generate large message overhead for building the global view and impose inconstant (usually very large) storage load on the central controller. Because every node has to access the central controller to retrieve its motion plan, these algorithms can generate communication bottleneck and may suffer from single point failure. Besides, they are not adaptive to node failures unless the global view is refreshed in a timely manner (frequent refreshment will however bring larger or even unacceptable message overhead). In these cases, centralized algorithms are not suitable for MSNs.

\section{Distributed algorithm}

Unlike its centralized counterpart, a distributed scheme $[36,59,78,84,88]$ does not require or build a global map. It enables nodes to collaboratively make their movement decisions using limited knowledge in a decentralized manner. Oftentimes, it adopts certain distributed global structure such as Voronoi diagram or spanning tree, whose construction requires the participation of every network node though, to facilitate nodes' decision making. The structure is distributed in that each node does not need to know the entire structure but how it is involved 
in the structure. In particular, if a node's movement decision is made by other nodes rather than by itself, the algorithm is called quasi-distributed.

Distributed algorithms are obviously more advantageous and more desirable than centralized ones for MSNs. Nevertheless, considering the infrastructurelessness network nature and unpredictable and incidental node failures, the cost of constructing and maintaining a distributed global structure may still not be acceptable. Specially, in quasi-distributed algorithms, tolerating node failures will be a major problem, since nodes' deployment decisions are made by some other nodes that could fail at any time.

\section{Localized algorithm}

A localized scheme $[33,36,38,52,59,62]$, where simple local behaviors achieve a global objective, is distributed in nature. It differs from traditional distributed algorithms in that it uses no distributed structure and generates no global computation or communication. It has inherent advantages in message complexity, scalability and adaptivity to topological change, especially in large-scaled failure-prone MSNs.

In such an algorithm, each node makes its movement decisions independently, merely using its $k$-hop neighborhood information such as neighbors' location and residual energy for a constant $k$. When $k=1$, the algorithm is called strictly localized. Among recently developed mobility-controlled coverage improvement strategies, localized algorithms hold the best prospect for fault-tolerance and energy efficiency.

\subsubsection{Design considerations}

The objective of a sensor self-deployment algorithm (or a sensor relocation protocol) is to achieve a maximized hole-free coverage out of a randomized node distribution (resp., to replace each individual failed sensor with a predefined redundant one without changing network topology) through strategic node movement. Additional constrains such as node density [33], coverage focus [45], node degree [62] and time delay [80] may however apply, depending on 
system or application requirements.

A mobility-controlled coverage improvement scheme (whether a sensor self-deployment algorithm or a sensor relocation protocol) should run in an asynchronous and localized manner. "Asynchronous" implies that it requires no synchronization for computation, communication, or node movement. Considering sensors' limited storage capacity, it must not generate inconstant per node storage load.

Considering network dynamics, it should not rely on any pre-knowledge of the network such as network boundary and network size. As sensors are usually dropped in an unknown environment in a random way, any terrain information about the sensory field should not be assumed either, and in this case, the scheme is expected to enable sensors to detect and avoid physical obstacles during the course of their movement.

Because sensors' physical movement (including starting their still motors) consumes much more energy than their computation and communication behavior, the scheme is expected to yield a minimized number of moves and minimized total moving distance while keeping communication overhead as low as possible.

\subsection{Motivation}

As a mobility-controlled coverage improvement technique, sensor self-deployment has been studied on an on-going basis for several years. A number of solution algorithms, e.g., $[33,36,38,52,78,84,88]$, to mention a few, were proposed in the literature. To our knowledge, they all emphasize only on coverage formation over a ROI (whereas, other type of coverage formation exists, as identified in this thesis); none of them operate in a localized manner and yet provide coverage guarantee, i.e., guarantee on maximized hole-free coverage. In addition, these algorithms have major drawbacks such as unrealistic assumptions (e.g., initial connectivity out of randomized node placement or fixed network size), requirement for global or network-wide computation and communication (e.g., for Voronoi diagram construction, clus- 
tering, or tree construction) and thus a large amount of message overhead, and/or vulnerability to topological change (e.g., due to node failure or node movement).

At the time of this writing, only two distributed sensor relocation protocols [79] and [80] were proposed for coverage maintenance in MSNs. They are both inferior for possible applications, compared to the protocols proposed in this thesis, for variety of reasons. Both of them rely on global/network-wide message transmissions for discovering replacement sensors, generating $O\left(n^{\prime} \sqrt{n}\right)$ (or even $O\left(n^{\prime} n\right)$ ) messages, where $n^{\prime}$ and $n$ are respectively the number of redundant sensors and the number of non-redundant sensors. They both require inconstant per node storage load $O\left(n^{\prime}\right)$. Further, the first protocol [79] has inconstant migration delay and unbalanced energy usage; the second [80] depends on the assumption of the pre-knowledge of the border of the coverage region. Both protocols do not address the issue of guaranteed discovery of a replacement sensor when one in fact exists and is connected to the area where it could move.

As addressed above, the issue of mobility-controlled coverage improvement has not yet been well investigated. Relevant research is still on its initial stage where defining problem, completing existing techniques, finding new techniques extendable to future relatively complex protocols are the main tasks. The immaturity of the research and the incompleteness of previous work spark our interest and motivate this thesis presented here.

\subsection{Contributions}

\subsubsection{Literature review}

In Chapter 2, we conduct and present a comprehensive, yet detailed, survey of existing sensor self-deployment algorithms and sensor relocation protocols in the literature, highlighting their features, strengths and weaknesses. We also review some other previous work relevant to this thesis. 


\section{Relevant publications}

- "Sensor placement in wireless sensor and actuator networks". Chapter 10, Wireless Sensor and Actuator Networks: Algorithms and Protocols for Scalable Coordination and Data Communication (X. Li, H. Liu, A. Nayak, and I. Stojmenovic). Approved by Wiley, 2008.

- X. Li, K. Lu, N. Santoro, I. Simplot-Ryl, and I. Stojmenovic. "Alternative Data Gathering Schemes for Wireless Sensor Networks". In Proceedings of International Conference on Relations, Orders and Graphs: Interaction with Computer Science (ROGICS), pp. 577-586, Mahdia, Tunisia, 2008.

\subsubsection{Sensor self-deployment}

In Chapter 3, we formally introduce the problem of focused coverage formation around a Point of Interest (POI), and define an evaluation metric, coverage radius. We assume that every node knows about its own location and the position of the POI, and that they obtain the status information (including position) of their one-hop neighbors from lower layer protocols. We devise two solution protocols, Greedy Advance $(G A)$ and Greedy-Rotation-Greedy $(G R G)$, which are to our knowledge the first sensor self-deployment algorithms that operate in a purely localized manner and yet provide coverage guarantee on size maximization and hole absence. The two algorithms both drive sensors to move along a locally-computed equilateral triangle tessellation (TT) to surround the POI. In GA, nodes greedily proceed as close to the POI as they can; in GRG, when their greedy advance is blocked, nodes rotate around the POI along a hexagon to a TT vertex where greedy advance can resume. Thanks to their strictly localized nature, the two algorithms work regardless of network disconnectivity and adapt to runtime node failure. We prove their correctness and analyzed their coverage radius property. Our study shows that GRG guarantees optimal hexagonal coverage radius, and optimal or near optimal (with a factor in $[0.88,1]$ ) circular coverage radius. Through extensive simulation, we 
also evaluate their performance on convergence time, energy consumption and node collision

GRG yields an optimal hexagonal focused coverage, which is however not guaranteed to be optimal in terms of circular coverage radius. Hence, In Chapter 4, we optimize GRG to achieve guaranteed circular coverage radius maximization. The optimized version of GRG is called GRG ${ }^{\star}$. It follows the same combined greedy-rotation idea as GRG. It differs from GRG in that it guides sensors to rotate along, rather than hexagons, polygons that best approximates circles over the TT graph. We evaluate $\mathrm{GRG}^{\star}$ comparatively with GRG through an extensive set of simulation.

\section{Relevant publications}

- X. Li, H. Frey, N. Santoro, and I. Stojmenovic. "Sensor Self-deployment for Guaranteed Circular Coverage Radius Maximization". Submitted for conference publication, 2008.

- X. Li, H. Frey, N. Santoro, and I. Stojmenovic. "Focused Coverage by Mobile Sensor Networks". Submitted for conference publication, 2008.

- X. Li, H. Frey, N. Santoro, and I. Stojmenovic. "Localized Sensor Self-Deployment with Coverage Guarantee". ACM SIGMOBILE Mobile Computing and Communications Review (MC $\left.{ }^{2} \mathrm{R}\right), 12(2): 50-52,2008$.

- X. Li, H. Frey, N. Santoro, and I. Stojmenovic. "Localized Self-Deployment of Mobile Sensors for Optimal Focused-Coverage Formation”. Technical Report, TR-2007-13, SITE, University of Ottawa, December 2007.

\subsubsection{Service discovery}

In Chapter 5, we formalize the distance-sensitive service discovery problem in WSNs. We assume that nodes are aware of their geographic position. We propose a novel localized solution algorithm, iMesh, which uses no global communication/computation and generates 
constant per node storage load. In iMesh, new service providers publish their location information in four directions, updating an information mesh. The information propagation for relatively remote service is restricted by an existing blocking rule, which also updates the mesh structure. Based on a new extension rule, nodes along mesh edges may further advertise newly arrived relatively near service by backward distance-limited transmissions, replacing previously closer service location. The final information mesh is a planar structure constituted by the information propagation paths. It stores the location of all the service providers and serves as service directory. Service consumers conduct a lookup process restricted within their home mesh cells to discover nearby services. We first analytically study the properties of iMesh including construction cost and distance sensitivity over a grid network model. Then we evaluate its performance in randomized sensor network scenarios through extensive simulation. Simulation results indicate that iMesh guarantees nearby (closest) service selection with very high probability $>99 \%$ (resp., $>95 \%$ ) at considerably low message cost.

\section{Relevant publications}

- X. Li, N. Santoro, and I. Stojmenovic. "Localized Distance-Sensitive Service Discovery in Wireless Sensor Networks". Submitted for journal publication, 2008.

- X. Li, N. Santoro, and I. Stojmenovic. "Localized Distance-Sensitive Service Discovery in Wireless Sensor Networks". In Proceedings of the 1st ACM International Workshop on Foundations of Wireless Ad Hoc and Sensor Networking and Computing (FOWANC), pp. 85-92, Hong Kong, China, 2008.

- X. Li, N. Santoro, and I. Stojmenovic. "Localized Distance-Sensitive Service Discovery in Wireless Sensor Networks". Technical Report, TR-2007-10, SITE, University of Ottawa, May 2007. 


\subsubsection{Sensor relocation}

In Chapter 6, we presented two localized sensor relocation protocols ZONER and MSRP for MSNs that are connected and contain pre-determined redundant sensors. ZONER is an application of the quorum-based location service [48,72,74]; MSRP is an application of our proposed algorithm iMesh. In ZONER, redundant sensors distribute their location information within their vertical registration zones; two specified neighbors of a failed node query sensors within their bounded horizontal request zones for a nearest redundant node; discovered redundant node is relocated as replacement in a shifted way to the position of the failed node along a natural migration path. In MSRP, redundant sensors chose nearest non-redundant sensors as proxy, which run algorithm iMesh on their behalf to construct an information mesh; four specified neighbors of a failed node search in four directions and discover nearby proxy nodes when hitting the perimeter of its home cell; the nearest delegated redundant sensor of a discovered closest proxy node is relocated in a shifted way to replace the failed node along an energy-aware migration path. We show that both ZONER and MSRP are superior to existing sensor relocation protocols for their localized message transmission, zero requirement of pre-knowledge of the network, and guaranteed node replacement. Moreover, MSRP also outperforms similar algorithms including ZONER for its novel localized migration path discovery.

\section{Relevant publications}

- X. Li, N. Santoro, and I. Stojmenovic. "Mesh-based Sensor Relocation for Coverage Maintenance in Mobile Sensor Networks". In Proceedings of the 4th International Conference on Ubiquitous Intelligence and Computing (UIC), LNCS 4611, pp. 696-708, Hong Kong, China, 2007.

- X. Li and N. Santoro. "ZONER: A ZONE-based Sensor Relocation Protocol for Mobile Sensor Networks." In Proceedings of the 6th IEEE International Workshop on Wireless Local Networks (WLN), pp. 923-930, Tampa, U.S., 2006. 


\section{CHAPTER 2 LITERATURE REVIEW}

$\mathrm{T}$ he problem of mobility-controlled coverage improvement consists of two sub-problems, sensor self-deployment and sensor relocation. The first solution schemes are sensor self-deployment algorithms proposed by Howard, Mataric and Sukhatme [37, 38] in 2002. From then on, it has been an active subject in MSNs. In this chapter, we survey major research efforts devoted to the mobility-controlled coverage improvement in the literature at length, highlighting their features, strengths and weaknesses.

In addition, we introduce two important previous work, Greedy-Face-Greedy routing [15, $27]$ and quorum-based location service $[48,72,74]$, which will later serve as a building block of some of our proposed algorithms in this thesis. We as well introduce some other location services and data centric storage schemes that are adoptable for replacement discovery in sensor relocation. 
Table 2.1: Algorithm comparison

\begin{tabular}{|c|c|c|c|c|c|c|c|c|c|}
\hline \multirow{2}{*}{ Tech. } & \multirow{2}{*}{ Protocol } & \multirow{2}{*}{ Class. } & \multicolumn{3}{|c|}{ Key Assumptions } & \multicolumn{3}{|c|}{ Coverage Properties } & \multirow{2}{*}{$\begin{array}{c}\text { Obstacle }^{*} \\
\text { Avoidance }\end{array}$} \\
\hline & & & Connectivity & Ranges & \# of nodes & Maximized & Hole-free & Shape & \\
\hline \multirow{8}{*}{$\mathrm{VC}$} & HMS-1 [38] & L & & 一 & & & & - & $\sqrt{ }^{*}$ \\
\hline & VFA [88] & Q & $\sqrt{ }$ & N/A & & & & - & \\
\hline & VEC [78] & D & $\sqrt{ }$ & - & $\sqrt{ }$ & & & - & $\sqrt{ }^{*}$ \\
\hline & DSSA [36] & $\mathrm{L}$ & $\sqrt{ }$ & - & $\sqrt{ }$ & & & - & $\sqrt{ }^{*}$ \\
\hline & IDCA [36] & L & $\sqrt{ }$ & - & $\sqrt{ }$ & & & - & $\sqrt{ }{ }^{*}$ \\
\hline & ATRI [52] & $\mathrm{L}$ & $\sqrt{ }$ & $r_{c} \geq \sqrt{3} r_{s}$ & & & & - & $\sqrt{ }^{*}$ \\
\hline & GGCL [33] & $\mathrm{L}$ & & $r_{c} \geq \sqrt{3} r_{s}$ & & & & - & $\sqrt{ }^{*}$ \\
\hline & NET [62] & L & $\sqrt{ }$ & - & & & & - & $\sqrt{ }^{*}$ \\
\hline $\mathbb{N}$ & HMS-2 [37] & $\mathrm{C}$ & $\sqrt{ }$ & $r_{\mathrm{c}} \geq r_{\mathrm{s}}$ & & $\sqrt{ }$ & $\sqrt{ }$ & - & $\sqrt{*}$ \\
\hline \multirow{3}{*}{ VD } & VDDA [36] & D & $\sqrt{ }$ & - & & & & - & \\
\hline & VOR [78] & D & $\sqrt{ }$ & - & $\sqrt{ }$ & & & - & \\
\hline & MinMax [78] & D & $\sqrt{ }$ & - & $\sqrt{ }$ & & & - & \\
\hline LB & SMART [84] & $Q$ & $\sqrt{ }$ & $r_{c} \geq \sqrt{\frac{5}{2}} r_{s}$ & & $\sqrt{ }$ & & square & \\
\hline MF & CBMX [18] & $\mathrm{C}$ & $\sqrt{ }$ & - & $\sqrt{ }$ & $\sqrt{ }$ & & square & \\
\hline \multirow[t]{2}{*}{$\mathrm{SC}$} & SDR [59] & $\mathrm{L}$ & & - & & & & - & \\
\hline & OSD [59] & $Q$ & $\sqrt{ }$ & $r_{c} \geq \sqrt{2} r_{s}$ & & $\sqrt{ }$ & $\sqrt{ }$ & square & \\
\hline BA. & REA [64] & $\mathrm{C}$ & $\sqrt{ }$ & - & $\sqrt{ }$ & $\sqrt{ }$ & & - & \\
\hline
\end{tabular}

L - localized; D - distributed; Q - quasi-distributed; C - centralized.

* Nodes are equipped special equipments by which they can detect physical obstacles.

\subsection{Sensor self-deployment algorithms}

In the following, we will go through existing sensor self-deployment algorithms in the literature. These algorithms are systematically organized in accordance with their employed self-deployment techniques. They by default assume the binary sensing model (see Section 1.1.3 in Chapter 1), unless clarified otherwise. Recall that $r_{s}$ and $r_{c}$ respectively represent nodal sensing radius and nodal communication radius. A summarized algorithm comparison can be found in Table 2.1 .

\subsubsection{Vector-based approach}

The well-known sensor self-deployment approach is the vector-based approach. Although the algorithms in this category may claim that they are inspired by different physical models 


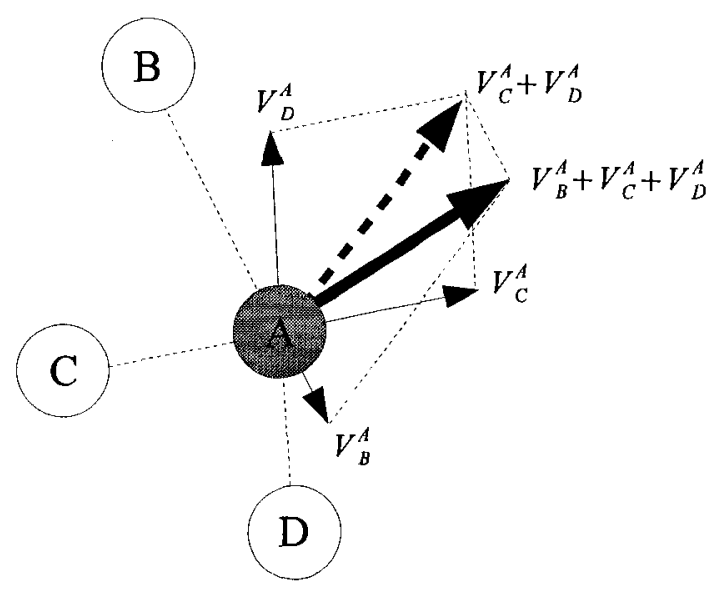

Figure 2.1: An example of movement vector

such as potential field [38], molecules [36], electro-magnetic particles [78], and compressible fluids [61], they share a common philosophy, that is, each node computes movement vectors for its neighbors based on their relative position and then move according to the vector summation; after a number of rounds of movement, the network reaches a equilibrium status, which produces a near uniform node distribution. Figure 2.1 illustrates the computation of movement vector. In this example, the movement sub-vector of node $A$ due to neighbors $B, C$ and $D$ are respectively $V_{B}^{A}, V_{C}^{A}$ and $V_{D}^{A}$.

The strength of this approach is that it enables nodes make their deployment decision independently using only local knowledge. However, due to randomized initial node placement, it provides no guarantee on sensing hole absence. Because the disappearance of any node breaks the equilibrium and triggers a chain of node movement (possibly network-wide) to recover, it can cause frequent topology change. In the following, we first present two typical algorithms in detail and then introduce several other algorithms of this type in a summarized form.

\section{A localized algorithm}

Howard, Mataric and Sukhatme [38] proposed a localized potential field bàsed sensor self-deployment algorithm under the assumption that, every node has the ability to measure the range and bearing of its nearby nodes and seeable obstacles. In this algorithm, a node 
receives from a potential field generated by other nodes and seeable obstacles virtual repulsive force, whose strength and direction depend only on the relative position of the force sources to the node. A node is driven by its received virtual force to move. When the virtual force exerted on a node is zero, the node stops moving. when all the nodes stay still, the network reaches a static equilibrium status. The core of this algorithm is composed of a virtual force function by which a node computes its received virtual force, a motion equation by which the track of a moving node is defined, and a control law by which virtual force is translated to the physical control to nodes' motors.

The virtual force $F$ exerted on a node $s$ by a scalar potential field $U$ is defined as the gradient of $U$, i.e., $F=-\nabla U$. The potential field $U$ is composed of two parts, the field $U_{0}$ due to obstacles and the field $U_{n}$ due to other nodes, and the partial force generated by these two parts are respectively $F_{0}$ and $F_{n}$. Then, $U=U_{0}+U_{n}$ and $F=F_{0}+F_{n}$. By considering that each obstacle carries an electric charge, an 'electrostatic' potential expression is defined as: $U_{0}=k_{0} \sum_{i} \frac{1}{r_{i}}$, where $k_{0}$ is a constant factor indicating the strength of the field, $r_{i}=\left|x_{i}-x\right|$ is the Euclidean distance between node $s$ at position $x$ and a seeable obstacle $i$ at position $x_{i}$. This expression indicates the force generated by $U_{0}$ at the point $x$. Hence, $F_{0}=-k_{0} \sum_{i} \frac{1}{r_{i}^{2}} \cdot \frac{\mathbf{r}_{i}}{r_{i}}$, where $\mathbf{r}_{i}=x_{i}-x$. Similarly, $U_{n}=-k_{n} \sum_{i} \frac{1}{r_{i}}$ and $F_{n}=-k_{n} \sum_{i} \frac{1}{r_{i}^{2}} \cdot \frac{\mathbf{r}_{i}}{r_{i}}$ where $\mathbf{r}_{i}$ is the relative position of node $i$.

The motion equation is defined as $\ddot{x}=\frac{F-F_{f}}{m}$ where $\ddot{x}$ represents the acceleration of the node, $m$ stands for the node's virtual mass, and $F_{f}$ denotes the friction that the node receives. The purpose of the use of the concept of friction is to ensure the stop of nodal movement. $F_{f}$ is computed by $F_{f}=v \dot{x}$, where $v$ is the pre-defined viscosity coefficient and $\dot{x}$ indicates the moving speed of the node. The control law is described as follows: $\Delta v \longleftarrow \frac{F-v v}{m} \cdot \Delta t$, where $v$ stands for the velocity of the node at certain time point $t, \Delta v$ stands for the change between the velocity of the node between time $t$ and $t+\Delta t .-a_{\max } \leq \Delta v \leq a_{\max }$. In fact, it defines how the velocity of a node is affected by the virtual force that it receives. The velocity of the node at $t+\Delta t$ is $v \longleftarrow v+\Delta v \quad\left(-v_{\max } \leq v \leq v_{\max }\right)$. 


\section{A quasi-distributed algorithm}

Zou and Chakrabarty [88] proposed a quasi-distributed sensor self-deployment scheme that uses a probabilistic sensing model. In this scheme, nodes form clusters, and cluster heads execute a virtual force based sensor self-deployment algorithm for its cluster members. During the execution, no node movement is actually performed; nodes move virtually in the memory of their cluster heads instead. For any node, all the transient locations it virtually visited are recorded by its cluster head. To save energy, whenever the distance from the node's initial location to current virtual location is beyond a predefined threshold value, the virtual force it receives is disabled, and thus it will no longer move. After the termination of the algorithm, a search procedure is executed by the cluster head to find the location that maximizes coverage, and then the node is informed to actually move to its final position by one step. This scheme impacts large computation and storage overhead on cluster heads and is apparently susceptible to cluster head failures. Below we will go through the virtual force based self-deployment algorithm employed by this scheme in detail.

In this scheme, each node in the network acts as a virtual force source to other nodes. The virtual force that a node exerts on another node can be either attractive or repulsive depending on the distance between the two nodes. Let $\left|s_{i} s_{j}\right|$ represent the Euclidean distance between two nodes $s_{i}$ and $s_{j} . d_{t h}$ is a pre-defined threshold value for nodal separation. If $\left|s_{i} s_{j}\right|>d_{t h}$, the force $\vec{F}_{i j}$ between $s_{i}$ and $s_{j}$ is attractive; if $\left|s_{i} s_{j}\right|<d_{t h}, \vec{F}_{i j}$ is repulsive; if $\left|s_{i} s_{j}\right|=d_{t h}, s_{i}$ and $s_{j}$ will not exert force on each other. In addition to the force from nodes, node $s_{i}$ also receives force from obstacles, which is repulsive and referred to as $\overrightarrow{F_{i R}}$, as well as the force from preferential areas, which is attractive and referred to as $\vec{F}_{i A}$. Therefore, the total force $\vec{F}_{i}$ exerted on $s_{i}$ can be defined as $\vec{F}_{i}=\vec{F}_{i R}+\vec{F}_{i A}+\sum_{j=1, j \neq i}^{k} \vec{F}_{i j}$. With this approach, nodes are able to actively move to preferential areas, intentionally avoid obstacles, and meanwhile keep a moderate distance(which is actually controlled by $d_{t h}$ ) from each other.

To reflect the uncertainty $r_{e}$ of sensor detection in real world, the coverage of a certain point $P(x, y)$ by node $s_{i}$, denoted by $c_{x y}\left(s_{i}\right)$, is treated as the probability that point is covered 
by $s_{i}$. Let $a=\left|s_{i} P\right|-\left(r-r_{e}\right) \cdot c_{x y}\left(s_{i}\right)=1$ if $r+r_{e} \leq\left|s_{i} P\right| ; c_{x y}\left(s_{i}\right)=e^{-\lambda a^{\beta}}$ if $r-r_{e}<\left|s_{i} P\right|<r+r_{e}$; $c_{x y}\left(s_{i}\right)=1$ if $r-r_{e} \geq\left|s_{i} P\right|$. The algorithm determines the coverage of a overlapped region $S_{o v}$ by the following equation: $c_{x, y}\left(S_{o v}\right)=1-\prod_{s_{i} \in S_{o v}}\left(1-c_{x, y}\left(s_{i}\right)\right)$ where $S_{o \nu}$ is the set of nodes that overlap $S_{o v}$. The rightmost part of the equation stands for the probability that $P$ is not covered by any node. Hence, by adjusting the distance threshold $d_{t h}$, a desired coverage at every point in the network can be achieved.

\section{Other similar algorithms}

Wang, Cao, and La Porta [78] proposed a distributed vector-based algorithm (VEC). This algorithm is not localized because it uses Voronoi diagram (which requires global computation) to help algorithm converge. It requires the pre-knowledge of the boundary of the target area and the size of the network. It run round by round until termination condition is met. In each round, nodes first broadcast their position and then construct their local Voronoi polygons based on received neighborhood information. Then they are driven by virtual force to move from densely covered areas to sparsely covered areas. Each node independently computes the average nodal separation in the case of uniform distribution and takes it as reference to computer virtual force. When two nodes are too close to each other, the virtual force between them will push them to move away from each other to achieve the average nodal separation. In the case that one node covers its Voronoi polygon entirely, it will not move but still exert virtual force on other nodes. To keep nodes stay in the target area, field boundary exerts repulsive force on nodes. The overall force received by a node will be the vector summation of the virtual force from field boundary and all Voronoi neighbors of the node.

Heo and Varshney [36] proposed two localized virtual-force based sensor self-deployment algorithms: distributed self-spreading algorithm (DSSA) and intelligent deployment and clustering algorithm (IDCA). In the two algorithms, the pre-knowledge of network size and target field area is required. In DSSA, each node calculates its local density and the expected node density (the node density under uniform node distribution). For any pair of neighboring nodes, 
there exists a repulsive force in between, which pushes them to move away from each other. The overall force exerted on a node is the summation of the force between the node itself and its every neighboring node. So, it actually depends on both the distance between the node and each of its neighbors as well as the local density of the node. Each node in the network is driven to move by the virtual force from its neighbors until a force equilibrium is achieved. Eventually, the network goes into a static equilibrium status. IDCA takes energy consumption into consideration during the process of deployment. To save energy, IDCA postpones the movement of the nodes in the regions with proper node density until nodal movement stabilize to some extent. And, it allow a node to choose, according to the local node density, two different modes to stay in, peer-to-peer mode or clustering mode. When the local node density is close to the expected node density, the node will choose the clustering mode; otherwise, it will act in peer-to-peer mode. When a node is in peer-to-peer mode, it just follows algorithm DSSA. If a node is operating in clustering mode, its contribution to virtual force generation will be affected by its remaining energy level. The higher the remaining energy level, the more the contribution.

Poduri, Pattern, Krishnamachari and Sukhatme [62] discuss Neighbor-every-Theta graphs as generalizations of RNG, GG, Delaunay triangulations and other structures, using them to guide the deployment of mobile sensors. Their proposed algorithm is based on virtual potential fields. It tries to solve the following problem. Given $N$ nodes and a global network connectivity constraint, how can positions for the nodes be determined in a distributed manner so as to maximize sensing coverage area while satisfying the connectivity constraint. The algorithm involves constructing local virtual forces between neighboring robots to encode their desired motion and/or placement configuration. Repelling and attracting forces between robots are used. These forces have inverse square law profiles. Repelling force tends to infinity when distance between nodes decreases to zero, while attracting force tends to infinity when the distance increases to $r_{s}$ (sensing radius). These two forces, if both applied, settle at distance $r_{s}$. In the algorithm, nodes assign priorities to neighbors based on desired theta 
coverage condition and their contribution to coverage, and decide for each whether or not to apply repelling attracting or both forces. Algorithm remains incomplete despite clear hints on its behavior.

Ma and Yang [52] proposed an adaptive triangular deployment (ATRI) algorithm. In this algorithm, a node divides its transmission range into six sector areas and establishes a coordination system in each sector. For a section, it adjusts its location along the corresponding axis based on the location of the nearest neighbor to the node in the sector. If the distance between them is less than $r_{s} \sqrt{3}$, its movement is away from the neighbor, or towards the neighbor otherwise. After the movement vectors in all six sectors are obtained, they are converted to uniform coordinates and aggregated to obtain the total movement vector for the node. The nodes independently adjust its location this way in rounds. After a number of rounds of adjustments, the layout of the network will be close to an equilateral triangle layout, and the coverage area of the network is enlarged. Two strategies, the distance threshold strategy and movement state diagram strategy can be used to reduce oscillation during the deployment.

Garetto, Gribaudo, Chiasserini and Leonardi [33] recently proposed an event-driven selfdeployment algorithm (referred to as GGCL in this thesis). In this algorithm, nodes are driven by their received exchange force from neighbors, potential force from detected events and friction force. A node $k$ exerts exchange force, which is either attractive or repulsive depending on nodal separation, on another node $i$ iff $k$ is neighboring $i$ and there is no other node $k^{\prime}$ making $\left|k^{\prime} i\right|<|k i|$ and $\angle k i k^{\prime}<\frac{\pi}{6}$. This condition restricts the number of neighbors acting on node $i$ to 6 at most and force the final network to have a triangle tessellation layout. Potential force can also be attractive or repulsive depending on a node's detected event intensity. This force pulls distant nodes towards the event location and pushes nearby nodes away. By adjusting event intensity threshold, different node density can be achieved around the event location. Friction force is used to stop nodal movement so that an equilibrium status can be eventually reached. 


\subsubsection{Incremental approach}

Howard, Mataric, and Sukhatme [37] proposed a centralized incremental deployment algorithm for MSNs that are composed of homogeneous nodes with the ability to "see" their surroundings. Initially, all the nodes but one are considered undeployed, and the only deployed node serves as a starting point. Then the algorithm run in iterations. In each iteration, a central controller deploys a single node. After the algorithm terminates, a line of sight relationship between deployed nodes are maintained, and the coverage, i.e., the total area visible to the network nodes, of the network is maximized. Below we will introduce how the central controller deploys a node in an iteration.

The central controller gathers the sensor data from previously deployed sensors via wireless communication to construct an occupancy grid over the target field. In the occupancy grid, a cell is considered free if it contains no obstacle for sure, or occupied if it contains obstacles for sure, or unknown otherwise (i.e., if no knowledge about this cell is available, or if contradictory evidence about the cell's occupancy state exists).

Then, the central controller converts the occupancy grid to a configuration grid, where a cell is free if and only if all nearby (within certain pre-defined distance) cells are free, or occupied if at least one nearby cell is occupied, or unknown otherwise. Afterwards, the configuration grid is transformed to a reachability grid. During this process, in the configuration grid, a free cell is marked as reachable if there is some path composed only of free cells between this cell and the location of certain deployed node, or unreachable otherwise; any other cell is marked as unreachable.

Once the reachability grid is established, nodes can be deployed conservatively to a location between free and unknown space to minimize the overlapping of sensory field, or optimistically to a location where they can reduce unknown space most. During this process, the candidate cells may not be unique. Then different policies can be applied to guide the final selection of reachable cells, and thereby yielding different network topologies at the end.

After a node's target cell is determined, a shortest path through previously deployed nodes 


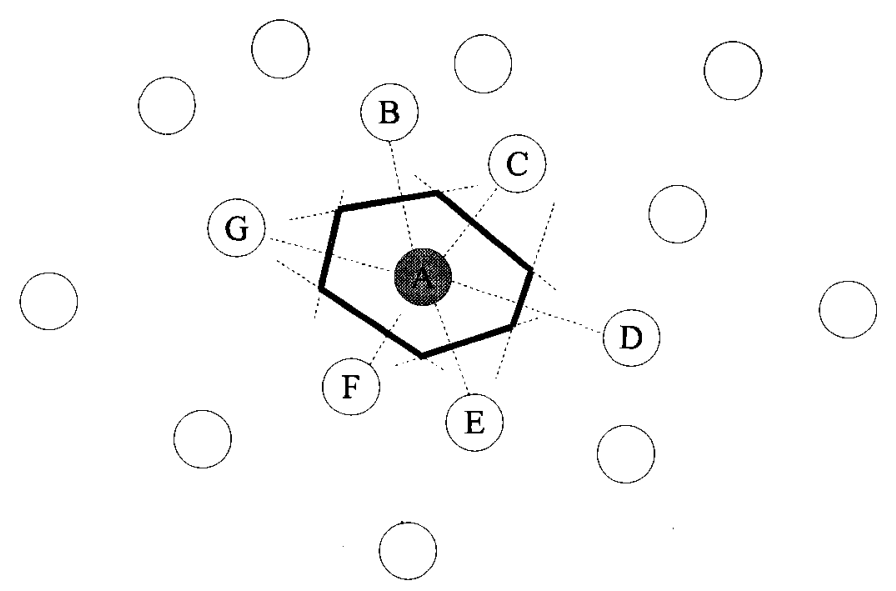

Figure 2.2: An example of Voronoi polygon

between the node and its target cell is discovered, and a sequential or concurrent shifted movement process is performed along the path: every node on the path move to the position currently occupied by the next node along the path towards the target cell.

\subsubsection{Voronoi-based approach}

Voronoi diagram [9] is a computational geometry structure widely employed in different fields. It partitions using $n$ given nodes a plane into $n$ Voronoi polygons, each of which contains exactly one node as generating node. A Voronoi polygon is constructed by the nearestneighbor rule such that every point in it is closer to its generating node than to any other node. In fact, it is the smallest one among the convex polygons formed by the bisectors between its generating node and other nodes, as shown in Figure 2.2 where thick lines constitute the Voronoi polygon of node $A$.

According to the proximity property of Voronoi diagram, if a sensor can not sense the events occurring at a location within its Voronoi polygon, nobody else is able to sense them, and therefore there must be a sensing hole at that location. In Voronoi-based sensor selfdeployment approach, sensors identify sensing holes within their Voronoi polygons and minimize local uncovered areas by aligning their sensing range with their Voronoi polygons. Usually, this approach involves multiple rounds of alignment and terminates when no more gain 
(e.g., utility gain in [36] and coverage gain in [78]) can be achieved. This heuristic approach unfortunately guarantees neither coverage maximization nor hole elimination. Several instance algorithms have been proposed in the literature. They differ merely in their node alignment methods.

Heo and Varshney [36] proposed algorithm VDDA. In this algorithm, the effective area of a node is defined as the intersection of its sensing range and its Voronoi polygon, and coverage is improved by increasing each node's effective area with minimal energy consumption. To do so, a node examines the points from its current location to the centroid of its Voronoi polygon, and then to the the midpoint of maximum and minimum along the $x$ and $y$ coordinates in the polygon; it moves to the point that maximizes its utility metric. A node's utility metric is defined as the product of the node's effective area and the node's estimated lifetime.

Wang, Cao, and La Porta [78] proposed VOR and MinMax. In the two algorithms, if a sensor finds a local sensing hole, it will move towards the Voronoi vertex; but if the moving direction is opposite to that of previous round, it stops for current round to avoid moving oscillations. Considering possible unknown distant Voronoi neighbors, the moving distance is restricted to the half of the communication range in VOR. In Minimax, in order not to generate new farthest Voronoi vertex, a node does not move as far as it does in VOR but to the so-called Minimax point, which is the center of the minimum circle going through two or three Voronoi vertices vertices and covering the rest.

\subsubsection{Load-balancing approach}

Yang, $\mathrm{Li}$ and $\mathrm{Wu}[84]$ treated sensor deployment as a load balancing problem and proposed a quasi-distributed scan-based sensor self-deployment scheme. In this scheme, the target field is partitioned into an $m \times m$ mesh. The nodes in a mesh cell form a cluster covering that cell. A cell containing no node is considered covered by an empty cluster. In a non-empty cluster, a node is elected as cluster head and required to take the responsibility to administrate the cluster and communicate with other clusters. In order to prevent cluster heads from energy overcon- 
sumption due to their extra work on cluster administration, the role of cluster head is taken in turn by every node in a cluster. The nodes in a cell is viewed as load. Empty clusters are first filled with redundant nodes of non-empty clusters using recursive doubling expansion in a pre-processing phase. Then, load balancing is performed along rows and columns in multiple rounds during a scan phase. Although this scheme can yield a nice uniform node distribution, it guarantees neither coverage maximization nor hole elimination. It generates large message overhead for cluster formation and multi-round load balancing, and it is susceptible to cluster head failure. Below we shall go through the details of this scheme.

During the pre-processing phase, segment expansion is performed to fill coverage holes. A segment $S_{i}$ is defined as the maximum sequence of non-empty clusters in a row or a column of the mesh. Denote by $W_{i}$ the total number of nodes in $S_{i}$ and by $C_{i}$ the length of $S_{i}$. $S_{i}$ recursively expands towards one direction by planting a seed (a node) in its neighboring empty clusters. The initial span of expansion is determined by $L_{i}=\left\lfloor\log C_{i}\right\rfloor$. Everytime when it expands, it doubles the span of its previous expansion. For example, if $C_{i}$ is $13\left(2^{3} \leq 13 \leq 2^{4}\right)$, then $L_{i}$ is 3 , and in this case, $S_{i}$ 's length first grows to $13+2^{3}=21$, and its length is then going to be $21+2^{4}=37$ after the second expansion. In order to perform $k$ expansions, the condition $W_{i} \leq 2^{L_{i}+k}+C_{i}$ must be satisfied. When $S_{i}$ meets another segment during its expansion, $W_{i}$ and $C_{i}$ need to be recalculated. After $S_{i}$ covers the last cluster or fails the expansion condition, the recursive doubling expansion stops, and an expansion towards the opposite direction may start if applicable.

After the pre-processing phase, the scan phase starts. The scan phase is executed in two rounds. Every mesh row is scanned and balanced in the first round, while every mesh column is processed during the second round. Consider a 1-D array $R$ of size $m$ which maps to either a mesh row and a mesh column. For the $i$-th cluster $H_{i}$ in $R$, its weight, i.e., the number of its hosted nodes, is denoted by $w_{i}$. The prefix weight sum of the first $i$ clusters is defined as $v_{i}=\sum_{j=1}^{i} w_{i}$. Then the total number of nodes in $R$ is $v_{m}$. So, the weight of a balanced cluster will be $\bar{w}=\left\lfloor v_{m} / m\right\rfloor\left(\right.$ or $\left.\left\lceil v_{m} / m\right\rceil\right)$. During a scan round, the algorithm first scans $R$ from 
one end to the other following the increasing order of cluster ID. In this scan, each cluster $H_{i}$ (actually its cluster head) computes $v_{i}$ and passes it to $H_{i+1}$, and the last cluster $H_{m}$ computes $\bar{w}$. Then $H_{m}$ triggers the another scan by sending $\bar{w}$ along $R$ back to the origin. In this scan, each $H_{i}$ can easily determine, according to its received $\bar{w}$ and its previously computed $v_{i}$, its status (overloaded or underloaded) and the number of nodes (load) to send to/take from each direction.

\subsubsection{Maximum-flow approach}

Chellappan, Bai, Ma, Xuan, and Xu [18] proposed a centralized minimum-cost maximumflow based sensor self-deployment algorithm. The authors consider a known number of mobility-limited flip-based sensors that are randomly dropped in a rectangular target field. After their initial placement, these sensors are able to move at most once, up to distance $F=k * d$ for some constant $k$, where $d$ is basic distance unit. In their proposed algorithm, the target field is partitioned evenly into a $R \times R$ grid, where $R$ is a pre-determined region size, and a centralized node (a base station) collects information about the number of sensors in each region (grid cell). The objective is to determine, based on sensors' initial location, an optimal sensor movement plan that maximizes the number of regions covered by at least 1 sensor and meanwhile minimize the total number of flips (moves). Mentionably, this algorithm was recently adopted for energy hole avoidance and network lifetime increase in [85].

The regions having at least one mobile sensor are considered as source, while those containing no sensor are called hole. The idea is to construct a virtual directed graph that records for each region the information of its hosted sensors and parameterizes the paths between regions based on the desired objective and mobility constraints, and then to convert the sensor self-deployment problem to the problem of maximizing the flow of sensors from source regions to hole regions without violating the path constraints between them and with minimized cost. As many maximum flow and minimum cost algorithms exist in the literature, the key problem is how to construct such a graph. The authors discuss graph construction in different 


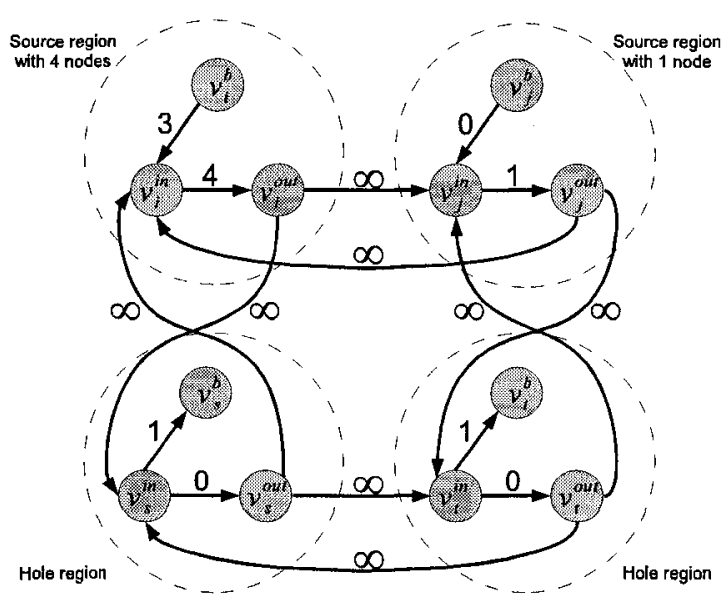

Figure 2.3: A virtual directed graph

cases: $F=R=d, F=d \wedge R>d$ and $F>d$. Below, we will introduce the case of $F=R=d$. The other cases can be obtained with simple modification over this case, and thus are omitted here. For details, one may refer to the original paper.

In the case of $F \geq d$, a region $i$ is represented by three vertices: a base vertex $v_{i}^{b}$ that tracks the number of nodes in the region, an in vertex $v_{i}^{\text {in }}$ that tracks the number of nodes that have flowed into the region, and an out vertex $v_{i}^{\text {out }}$ that tracks the number of nodes that has flowed out of the region. Let $n_{i}$ be the number of nodes in region $i$. An edge of capacity $n_{i}$ is added from $v_{i}^{\text {in }}$ to $v_{i}^{\text {out }}$, meaning up to $n_{i}$ nodes can flow out. If $n_{i} \geq 1$, an edge of capacity $n_{i}-1$ is added from $v_{i}^{b}$ to $v_{i}^{i n}$, meaning at least one node has to stay; otherwise, an edge of capacity 1 is added from $v_{i}^{i n}$ to $v_{i}^{b}$, meaning at most 1 sensor can flow in. For two neighboring regions $i$ and $j$, an edge of capacity infinity is added from $v_{i}^{\text {out }}$ to $v_{j}^{\text {in }}$ and from $v_{j}^{\text {out }}$ to $v_{i}^{\text {in }}$. This allows an unlimited number of sensors (if any) to move between the two regions. Note that the neighborship between two regions is depending on the flip distance. In this graph, the cost on inter-region edges is 1 , while the cost on intra-region edges is 0 . A virtual graph contains 4 nodes is shown in Figure 2.3. 


\subsubsection{Stochastic approach}

Mousavi, Nayyeri, Yazdani and Lucas [59] proposed a localized stochastic deployment routine (SDR). In this algorithm, sensors are dropped in a $X \times Y$ field, and they move from dense area to sparse area according to their local knowledge through guided random walk. The execution of the algorithm is independent of the initial connectivity of the network. Nevertheless, because of its stochastic nature, the algorithm provides no guarantee on coverage maximization, hole elimination, and connectivity in the final network. Below, we shall go through its details.

A sensor node moves at local time $t$ towards a location randomly and uniformly picked within a moving rectangle (MR), whose size exponentially decreases over time. For $t=0$, the MR has size $X \cdot p_{0}$ by $Y \cdot p_{0}$, where $p_{0}<1$ is a pre-defined constant; for $t>0$, its size is $X \cdot p_{0} \cdot p^{t}$ by $Y \cdot p_{0} \cdot p^{t}$, where $p<1$ is also a pre-defined constant. Restricted by its evershrinking $\mathrm{MR}$, the maximum moving distance of a sensor during a time unit monotonically decreases, which guarantees the algorithm's termination. Under this circumstance, the key will be the determination of the position of the MR. As you will see below, this is actually accomplished by a local process at each node on the fly.

Suppose that a node has $N$ neighbors ( $k$-hop neighbors for a constant $k$ ) in total. Let $N_{w}$ and $N_{e}$ be the number of neighbors that are located respectively on the west and the east of the node. Similarly, $N_{n}$ and $N_{s}$ are the number of nodes residing on the north and the source of the node. Then $N=N_{w}+N_{e}=N_{n}+N_{s}$. Further, let $d_{w}, d_{n}, d_{e}$ and $d_{s}$ be the distance from the node respectively to the west border, the north border, the east border and the south border of its MR. As a node is always expected to move to sparse area from dense area, the MR should be positioned in such a way that it covers a small part of the local dense area of the node. Hence, it is defined that $\frac{d_{w}}{d_{e}}=\frac{N_{e}}{N_{w}}$, and in this case, $\frac{d_{w}}{d_{e}+d_{w}}=\frac{N_{e}}{N_{w}+N_{e}}$, and therefore $d_{w}=\frac{N_{e}}{N} M R . x$ where $M R . x$ represent the width of the MR. Likewise, $d_{n}=\frac{N_{s}}{N} M R . y$.

Hence, given its own location and the size of the MR, a node is able to compute the position of the MR. And, Having known the position and the size of its MR, the node simply 


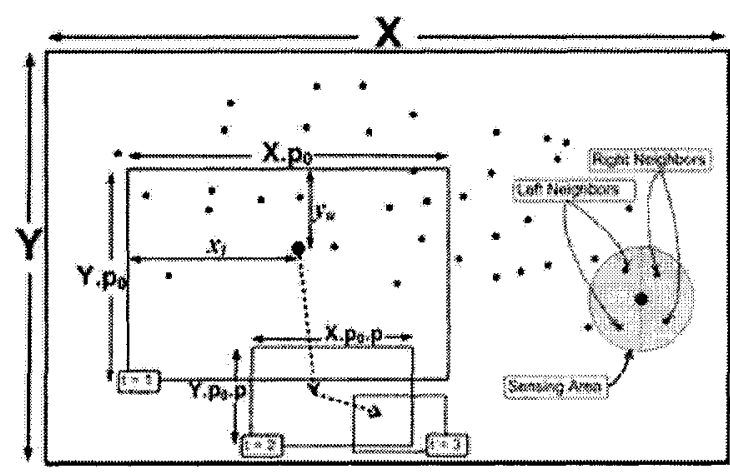

figure credit: figure is taken from [59]

Figure 2.4: MR-based stochastic movement

picks a target location within its MR probabilistically with uniform distribution and move to that location. This computation and movement is performed on a periodical basis. Finally, the the node stops moving when the size of its MR becomes too small, for example smaller than a threshold value. During their movement, two neighboring nodes may exchange their target location if they find that doing so will reduce their moving distance and thus energy consumption.

\subsubsection{Point-coverage approach}

Mousavi, Nayyeri, Yazdani and Lucas [59] proposed a simple tree-based One Step Deployment (OSD) algorithm. The algorithm partitions the target field into a 2D grid with edge length set to nodal communication range, and then translates the area coverage problem into a point coverage problem over the grid. It relies heavily on a rooted spanning tree and is quasi-distributed in that the deployment decision of a node (except the tree root) is not made independently by itself by by its tree parent. The main strength of this algorithm is its guarantee on hole-free coverage in failure-free environments. Nevertheless, it will not be the case in practical failure-prone MSNs. In fact, in the presence of node failures, the execution of the algorithm might be interrupted with even no output. The important fault-tolerance problem is however not addressed by the authors. Below we shall introduce the algorithm in detail. 
A breath-first tree rooted at greatest-ID node is built in the first place. What follows is a classic converge cast initiated by leaf nodes. In the converge cast, after receiving from all its children a message that contains the subtree size of the sender, a node computes the size of the subtree rooted at itself and sends the information to its parent. Thus after the converge cast, each node knows about the size of each of its breach. Thereafter, a recursive point assignment process starts. Specifically, the root choses grid point $(0,0)$ as its own deployment destination and assigns each of its subtrees a sub-area with a matching number of grid points. The root of each subtree subsequently does the assignment in the same way. This recursive assignment process stops when leaf nodes are reached. Finally, each node knows about its designated deployment point and then moves to it by one step.

\subsubsection{Genetic Algorithm approach}

Ramadan, El-Rewini, and Abdelghany [64] addressed the deployment of heterogeneous sensors as a combinatorial optimization problem. They consider a set $S$ of heterogeneous sensors that are designated to monitor a target field composed of a number of zones $A$ for a time horizon $T$. For every time interval $t \in T$, each sensor $s \in S$ is associated with a predefined time-evolving reliability $R_{s}^{t}$, and each zone $i \in A$ is assigned a time-varying weight function $w_{t}^{i}$ that defines the importance of the observations in the zone over $T$. Sensors may differ in a number of factors, e.g., lifespan, power-saving capability, mobility, sensing range, and communication range, etc., depending on application. Then the objective is to determine the optimal deployment scheme that maximizes the coverage of the target field. Coverage is considered maximized when the zones with highest weight are all monitored, and sensors with high reliability are assigned to high weight zones. Alternatively, coverage is also considered maximized when the number of monitored zones is maximized, and each zone is monitored by exactly one sensor at any time.

The authors proposed a centralized heuristic solution based on the the well known Genetic Algorithm (GA). GA simulates genes and nature selection. It is a powerful technique of 


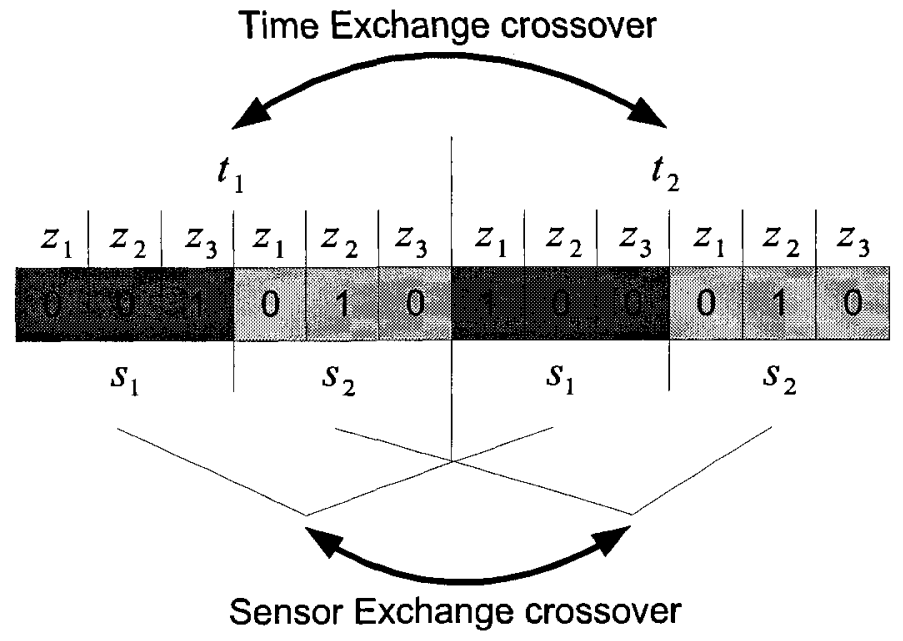

Figure 2.5: Crossover operations over a single chromosome

solving combinatorial optimization problems. In general, a GA algorithm first generates an initial set of chromosomes, and then it executes the following steps in iterations until certain stopping criteria are met: (1) Evaluation: evaluate the cost of each individual chromosome in current chromosome set; (2) Selection: determine the fitness of each individual chromosome in current chromosome set; (3) Reproduction: apply crossover and/or mutation operations (based on fitness) to current chromosome set to produce a new chromosome set, which serves as the input of next iteration.

In the proposed solution, a chromosome contains $|A| \cdot|T| \cdot|S|$ number of genes, each of which indicates a deployment plan for a sensor at certain time interval in certain zone. Specifically, if a sensor $s \in S$ is deployed at zone $i \in A$ at time interval $t \in T$, the corresponding gene is set to 1. According to its deployment plan, a sensor moves from zone to zone over time. The initial chromosome set of pre-defined size $n$ can be generated either randomly or following certain given rules. Two crossover operations, time exchange (TE) and best chromosome (BC), are defined. Both TE and $\mathrm{BC}$ are for exchanging the sensor deployment patterns in the same time interval in two different chromosomes. They differ in that, TE uses two randomly selected chromosome, while BC uses two fittest chromosomes. The fitness of each chromosome $x$ is measured using a fitness function: $F(x)=\sum_{t} \sum_{i} \sum_{s} w_{i}^{t} R_{s}^{t}$. Mutation operations, where some of 
the generated chromosome genes are randomly flipped from 0 to 1 or vice versa, are performed after crossover operations to prevent search dead-end and chromosome repetition. A newly generated chromosome is accepted if and only if it passes a feasibility check which considers various capability constraints of each sensor. The algorithm stops after a fixed number of iterations or after the solution no more improves.

A simple single single-chromosome-per-iteration mechanism can be alternatively used. In this case, a chromosome set contains only one element, and TE operation is for exchanging the sensor deployment patter in two randomly selected time intervals in the only chromosome. Since $\mathrm{BC}$ is no longer applicable, a new crossover operation sensor exchange (SE) is employed instead. By SE, the deployment pattern of two randomly selected sensors over the entire horizon $T$ is exchanged. The two crossover operations are illustrated in Figure 2.5.

\subsection{Sensor relocation protocols}

At the time of this writing, only two sensor relocation algorithms, a proxy-based protocol [79] and a grid-quorum based protocol [80] are proposed in literature. Below we will examine the two protocols in detail.

\subsubsection{Broadcast-based approach}

Wang, Cao and La Porta [79] proposed a proxy-based sensor relocation protocol (referred to as WCP) for the sensor networks composed of both static nodes and mobiles. In this protocol, mobile nodes periodically advertise within a predefined range; static nodes construct a Voronoi diagram and listen to mobile nodes' advertisements. Once a static node finds a sensing hole in its Voronoi polygon, it bids according to the hole size the best mobile node that it knows. A mobile node accepts a bid that is larger than its previously accepted bid and recently received any other bid, and moves to heal the corresponding sensing hole. By this means, it migrates to large holes from relatively small ones in iteration and stays still when finding no 
yet large hole. To save energy, it logically moves to a transient target location by choosing proxy and performs actual movement to the final location only. This protocol consumes a large amount of bandwidth for advertising and bidding mobile nodes, and it could cause that multiple mobile nodes move to fill the same sensing hole. In the following, we will go through the details of the iterative hole-healing process.

In an iteration, mobile nodes broadcast within a predefined radius their location information and base prices (initially set to zero) as service advertisement. After receiving a service advertisement, a static node records the encapsulated information to create a service provider (i.e., mobile node) list. Once a static node finds a sensing hole in its Voronoi polygon, it estimates the hole size, computes the bid, chooses a closest mobile node with lowest base price from its service provider list, and sends a bidding message to that node. In the case that a mobile node receives more than one bidding messages from different static nodes, a mobile node choose the highest bid and send a delegate message to the bidder. After receiving the delegate message, the bidder becomes the proxy of the mobile node.

A proxy node executes the protocol on behalf of its delegated mobile node. The mobile nodes having proxies cease to execute the protocol and wait for a movement notification from their proxies. As the hole-healing process iterates, the proxy node of a mobile node may change from one for a small sensing hole to one for a large sensing hole, rendering the mobile node logically migrating from one location to another. When a proxy node fails in finding larger sensing holes with respect to the base price of its delegated node, it will consider that its delegate node's current logical location is the final location, and then it informs its delegated node to physically move to that location.

To reduce moving distance, proxy nodes may exchange their delegated nodes. When a proxy node finds that its delegated node has to move a distance longer than a pre-defined threshold value, it searches its service provider list for such a proxy node that the moving distance of their delegated nodes will be both shorter than the threshold value if they exchange their delegated nodes. Then it will send an exchange message to that node (if any) and wait 
for a confirmation message. In the exchange message, the moving distance without delegated node exchange is specified so that the receiver is able to make its decision on the proposal.

When different static nodes detect the same sensing hole, they bids mobile nodes independently and possibly cause that multiple mobile nodes move to the same location. To avoid node collision, a proxy node tries to re-detect the sensing hole that its delegated node is going to heal. If the hole still exists, it will simply considers that there is no collision. Otherwise, the proxy node will further check if the moving distance of its delegated node is the shortest among those of other mobile nodes for the same hole. If so, it waits for other's giving-up; otherwise, it cancels the movement, set the base price of its delegated node to zero, and readvertise the new price in the next round.

\subsubsection{Quorum-based approach}

Wang, Cao, Porta and Zhang [80] proposed a grid-quorum-based relocation protocol (referred to as WCPZ). This protocol considers that each sensor node is associated with an application-dependent maximum recovery delay. It partitions the sensor field evenly into grids (and thus requires the pre-knowledge of the network boundary) and clusters nodes in each grid to simplify hole detection and node administration. It runs the quorum-based location service $[48,72]$ over the grids for redundant node discovery, and it uses the shifted migration method to relocate a redundant node to a sensing hole. This protocol generates large message overhead due to network-wide clustering and flooding-based relocation path discovery. It is vulnerable to void areas (e.g., due to node failure or unbalanced node distribution). The occurrence of void grid can lead to failures in both redundant node discovery (empty grid blocks message transmission) and relocation path discovery (pre-defined search range is not large enough to contain entire path). Below we will go through the details of this protocol.

In this protocol, the sensor field is partitioned evenly into a number of grids. A row of grids is called supply quorum, while a grid column is called demand quorum. In each grid, one node is elected as grid head and takes the responsibility to collect the location of all the grid mem- 
bers. A grid head identify redundant nodes and detects sensing holds within its grid Based on its grid members' location, and it publishes the information about the redundant nodes to all the grid heads in the supply quorum that it is residing in. When a grid head detects a sensing hole, it broadcasts a request within its residing demand quorum to discovery a closest redundant node. Because the demand quorum intersects with all the supply quorums, a redundant node can always be found if any exists. To reduce message complexity, the information about already discovered closest redundant node is piggybacked on the request message and used to restrict the distance that the request message may travel further.

To reduce relocation delay, the protocol uses the shifted migration method (see Section 1.2.1 in Chapter 1) to relocate sensors. Recall that each node is associated with a maximum recovery delay. A relocation path must guarantee that the delay requirements of its comprising nodes be satisfied. And, to balance power consumption, it should also guarantee that the difference between the total power consumption and the minimum remaining power of its comprising nodes be minimized. Expecting the relocation path to lie closely along the straight line from the redundant node to the sensing hole, the search for the relocation path is conducted during a flooding process confined within an elliptic zone. Note that, it is in fact difficult to pre-determine the size of the zone so as to guarantee the success of the search in the case that the relocation path deviates far from the line; on the other hand, setting the zone to the entire network can provide the guarantee but induces increased message overhead.

The grid head $s_{0}$ of the grid where the sensing hole exists initiates a flooding process in the search zone by a request message. The message contains the maximum recovery delay $T_{0}$, departure time $t_{0}=0$, the redundant node $s_{r}$, the total energy consumption $E_{0}=0$, and the minimum residual energy $E_{\min _{0}}=\infty$. After receiving the first request message, a node $s_{i}$, whose remaining energy level is $P_{i}$, determines a waiting list that is composed of its neighbors in the zone and towards the sensing hole. And, after receiving all the request messages from the nodes in its waiting list, it starts the following processing: for each node $s_{j}$ at distance $d_{i j}$ in its waiting list, it checks if $d_{i j} \leq\left(T_{j}+t_{j}\right) *$ speed (where speed stands for 
nodal moving velocity) and $P_{i}-d_{i j}>E_{\text {min }}$ both hold; then it choses as its predecessor the one that yields positive answer and minimizes the total energy consumption; afterwards, it updates the message with $T_{i}, t_{i}=\frac{T_{j}+t_{j}-d_{i j}}{\text { speed }}, E_{i}=E_{j}+d_{i j}, E_{\text {min }_{i}}=\min \left\{P_{i}-d_{i j}, E_{\text {min }_{j}}\right\}$ and broadcasts the message. When the message reaches the redundant node, a satisfactory relocation path is established, and all the nodes along the path are informed to move at their departure time.

\subsection{Other previous related work}

We shall now introduce two important work, i.e., Greedy-Face-Greedy (GFG) routing [15, 27] and quorum-based location service [48, 72,74], respectively in Sections 3.4.2 and 2.3.2. These two algorithms will be later used as basic techniques by our proposed distance-sensitive service discovery algorithm iMesh in Chapter 5 and sensor relocation protocols ZONER and MSRP in Chapter 6.

In Section 2.3.2, we also review some previous work on location service and data-centric storage in wireless ad hoc networks. Since the two separate topics are out of our interest, we do not intend to include all the existing work devoted to them. Rather, we focus on several solution schemes that could be adopted to accomplish the replacement discovery task in sensor relocation. A survey of data centric storage/location service can be found in $[28,75]$.

\subsubsection{Greedy-Face-Greedy routing}

Bose, Morin, Stojmenovic and Urrutia proposed a Greed-Face-Greedy routing protocol (GFG) for wireless sensor networks [15]. The GFG is a combination of a simple greedy forwarding strategy and the face routing technique. Since face routing is based on a planar graph, GFG requires a pre-processing phase to planarize the underlying network graph, which is modeled as a unit disk graph. Any planar graph may be used to support face routing in GFG as long as it can be constructed in a localized manner. One option is Gabriel Graph (GG). To construct GG, a node $u$ preserves every outgoing edge $u v$ that satisfies the condition: the 
diametral circle passing trough $u$ and $v$ contains no other neighbor nodes than $v$. Another possible option is Relative Neighborhood Graph (RNG). To construct RNG, a node $u$ preserves every outgoing edge $u v$ that satisfies the consideration: the lune area of the two circles, respectively centered at $u$ and $w$, with radius $|u v|$ contains no other nodes than $v$. As proven in [27], when GG or RNG is applied, greedy forwarding recovery in face mode is guaranteed when traversing the first face.

In a GFG routing process, greedy forwarding takes in charge of packet delivery whenever possible, while face routing is used only for passing packets around the void areas that block greedy forwarding. A node greedily forwards a packet towards the destination by choosing as the next hop its neighbor closest to the destination. In the case that the node itself is the one closest to the destination among its neighborhood, the packet is forwarded in face routing mode using right-hand/left-hand rule until the destination or a node yet closer to the destination is found. The right-hand rule is that, to traverse the interior (resp., exterior) of a face, a packet is forwarded in the counterclockwise (resp., clockwise) direction along the perimeter of the face. The left-hand rule is just the opposite of the right-hand rule.

There are two variant of the GFG. One employs the "before-crossing" scheme. Suppose a routing packet $P k t$ for destination node $t$ is switched to face routing mode at node $u$. Draw an imaginary line $\overline{u t}$ from $u$ to $t$. Line $\overline{u t}$ intersects a sequence of faces in the previously constructed GG (or RNG, or LDT). $u$ sends $P k t$ along the first face using right-hand (or, lefthand) rule. $P k t$ traverses along the face perimeter and reaches a node $v$. Before $v$ forwards $P k t$, it checks if the link, say $E$, that the packet is going to pass intersects the imaginary line $\overline{u t}$ at certain point $p$. If yes, it will, instead, sends $P k t$ to its incident link next to link $E$ in the clockwise direction, resulting in a face change. In the new face, $P k t$ is forwarded in the same way (i.e., using right-hand rule), and the next face change happens only at the edge intersecting the remaining line segment $\overline{p t}$. Face change ensures that the routing message progresses towards the destination. The other variant employs "after-crossing" scheme. This scheme is the same as the before-crossing except that face change happens after a routing 


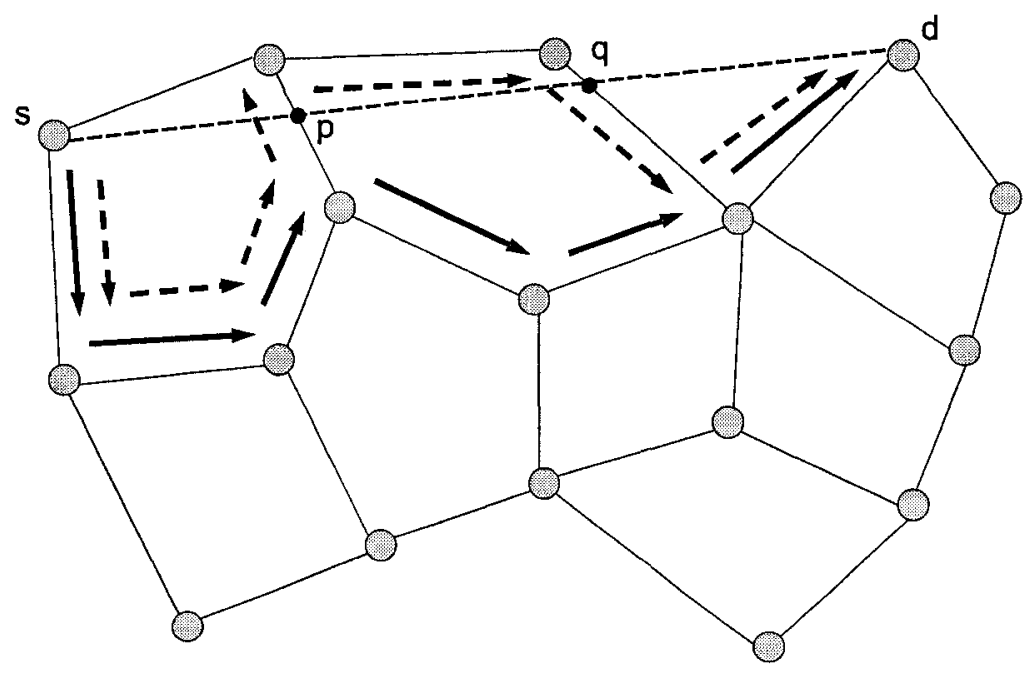

Figure 2.6: Face routing in GFG

packet passes the intersecting link, and that, in the new face, hand is changed as well, either from right-hand to left-hand or from left-hand to right-hand. Figure 2.6 gives an example of the face routing part of the GFG. In this figure, node $s$ wants to find a route to node $d$ solely by face routing. The route discovered by the before-crossing scheme is shown by solid arrows represent, while the route established using the after-crossing scheme is highlighted by dashed arrows.

GFG a stateless routing protocol in the sense that nodes do not need to remember any routing information such as routing table or route list. It is the first localized protocol that provides guaranteed packet delivery. There exist several other combined Greedy-Face routing protocols, e.g., GOAFR+ [42] and GPVFR [43], but they are in essence a variant of GFG. The drawbacks of GRG are that the face routing part may generate very long routes, and that the nodes around a void area may run out of battery power quickly because they are frequently used to route packets around the void area. 


\subsubsection{Location services and data centric storage schemes}

\section{Quorum-based location service}

Stojmenovic $[48,72]$ proposed a quorum-based location service to support geographic routing in wireless ad hoc networks. Each node monitors the state of its incident links. Whenever a link breakage/creation occurs (possibly due to its own or others movement), it reports its current position to its neighbors, which may or may not reply with their own location depending on whether the sender is a newly detected neighbor for them. After a certain number of link changes, the node forwards its current position to all the nodes located in a "column" of certain thickness. That is, it sends its location in both north and south direction to reach the north and south boundaries through GRG routing protocol [15]. The nodes along this column form an update quorum.

When a source node wants to communicate with a destination node, it has to search for the location of the destination if its local record about the destination is out of date. The source queries its $q$-hop neighborhood for the destination's location. If the answer is negative, or if the obtained information is not fresh enough, the search continues in the east and the west direction with certain thickness. One more request may be sent directly to the destination to take the advantage of the possible correctness of the best information obtained during the $q$ hop neighborhood search. The three searches are performed independently. The traces of the eastbound search and the westbound search respectively form a "row", i.e., a search quorum, which together intersects the update quorum of every other node.

As the query message travels along the search quorum, it picks the latest location information about the destination. After reaching the ends of the search quorum, it is forwarded to the destination, which then replies the source directly with correct location and possibly form a route for future data transmission. Alternatively, the intersection nodes of the search quorum of the source and the update quorum of the destination may reply immediately if their stored information about the destination is sufficiently fresh. For example, in Figure 2.7, $d$ sends its location update in a vertical quorum of thickness $1 ; s$ sends a query message in both east and 


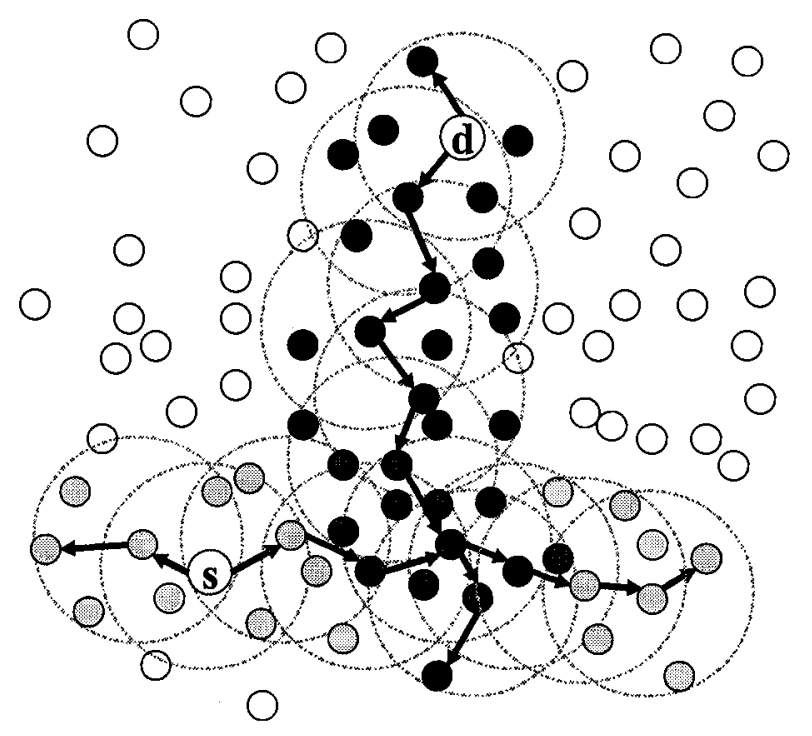

Figure 2.7: An example of the quorum-based location service

west directions. One of the two search quorums intersects the location update quorum of $d$. The nodes in the intersection area can provide $s$ with $d$ 's location.

By the quorum-based location service, every node can discover every possible destination without network-wide querying. Because location update and search is restricted within two strips, i.e., a vertical quorum and a horizontal quorum, communication overhead is greatly reduced. No particular node is designated to store a certain node's location, and thus no bottleneck is created in the network. If the destination is nearby the source, the source will be able to find the destination location quickly since their update quorum and search quorum intersect earlier than in the case that they are far apart.

Despite these advantages, this scheme has some unnegligible weaknesses. Location update still has to cross the entire network; to guarantee row-column intersection, the outer boundary of the network has to be included into any search quorum, making the boundary nodes' battery power drain out fast. If all the nodes are collinear in location update direction, every node is in every other nodes' update quorum and has to all their locations. In this case, the protocol may overload the sensor nodes with severe storage constraint. 


\section{Grid Location Service}

Li, Jannotti, De Couto, Karger and Morris proposed a distributed Grid Location Service (GLS) for large-scale ad hoc networks [44]. By the GLS, a sensor field is evenly partitioned into a number of grids. These grids are called order-1 square. Four neighboring order- $k$ squares form an order- $(k+1)$ square. To avoid overlapping, an order- $k$ square is required to be part of only one order- $k+1$ ) square. By this means, a multi-resolution grid hierarchy (actually a quad-tree) is established, and each node is located in exactly one square at each level of the hierarchy. This partitioning method serves as the base of the GLS and is known by every node.

Every node $u$ maintains its location information with a small set of location servers geographically distributed in the network, three at each lever of the grid hierarchy. Specifically, $u$ sends a location update message containing its location to its three adjacent order- $n$ squares using geographic forwarding. The first receiver node in such a square forwards, based on its own knowledge, the message to a node whose ID is closest in a circular ID space to $u$. This greedy type of message forwarding occurs at every intermediate node and is restricted within the square, and it will stop at a local minima, which is the location server of $u$ in the square.

Before a source $s$ performs geographic routing to a destination $t$, it queries one of the location servers of $t$ for $t$ 's location in a similar way as location update. $s$ sends a destination search message carrying its current location to a node, whose ID is closest to the destination's and for which it currently has location information. Each intermediate node greedily forwards the message in the same way. Eventually, the message will reach a location server of $t$. This location server then routes the message to $t$ by geographic forwarding, which then directly replies $s$ with its current location. To tolerate mobility, before a node moves to another order1 square, it leaves a "forwarding pointer" in its current order-1 square. This pointer is used to locate that node if a location query intended for the node arrives.

Figure 2.8 illustrates the work principle of GLS. In this example, node $B$, whose ID is 17 , choses its location servers, whose IDs are circled, in the grid hierarchy; two destination searches for node $B$ are originated respectively from node 76 and 90 , and their search trails 


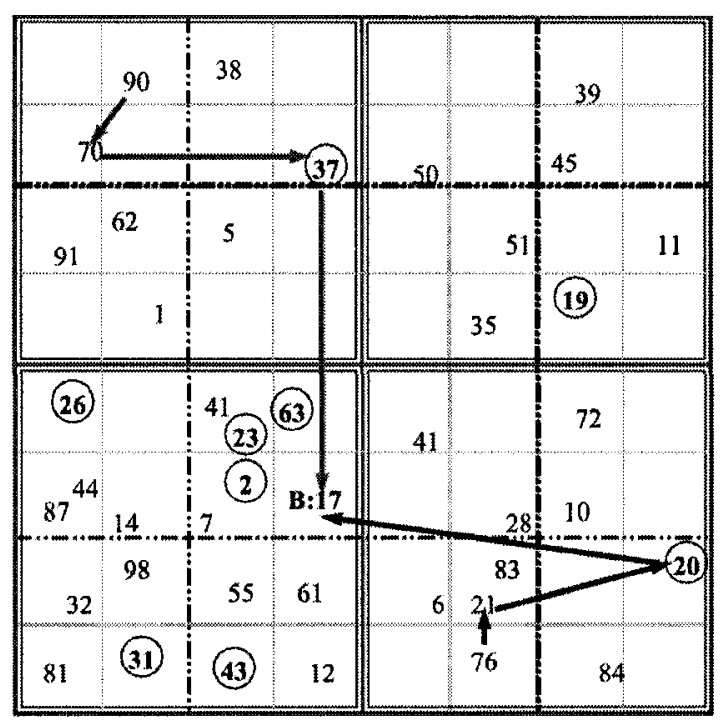

Figure 2.8: An example of the grid location service

are highlighted by arrowed lines.

GLS has two main advantages. One is that the use of multiple location serves for a single node prevents the occurrence of communication bottleneck and single point failure; the other is that queries for the location of a nearby node can be answered quickly since location servers are distributed densely near the node but sparsely farther away from the node. But it also has many disadvantages. First of all, pre-knowledge about the size of the sensor field is required for the grid partition. Secondly, the scheme may generate large degree of message overhead, because each node has to sends a location message to its every single location server respectively along different routes, and because location update and query travel along zigzag lines. Thirdly, the forwarding-pointer-based fault-tolerance method is not effective enough. For example, if all the nodes in an order-1 square moves out, nobody will store forwarding pointers for them, leading to query failures.

\section{Geographic Hash Table}

Ratnasamy, Karp, Yin and Yu [65] proposed a Geographic Hash Table (GHT) data-centric storage scheme for wireless ad hoc networks. In this scheme, an event is expressed by a value 


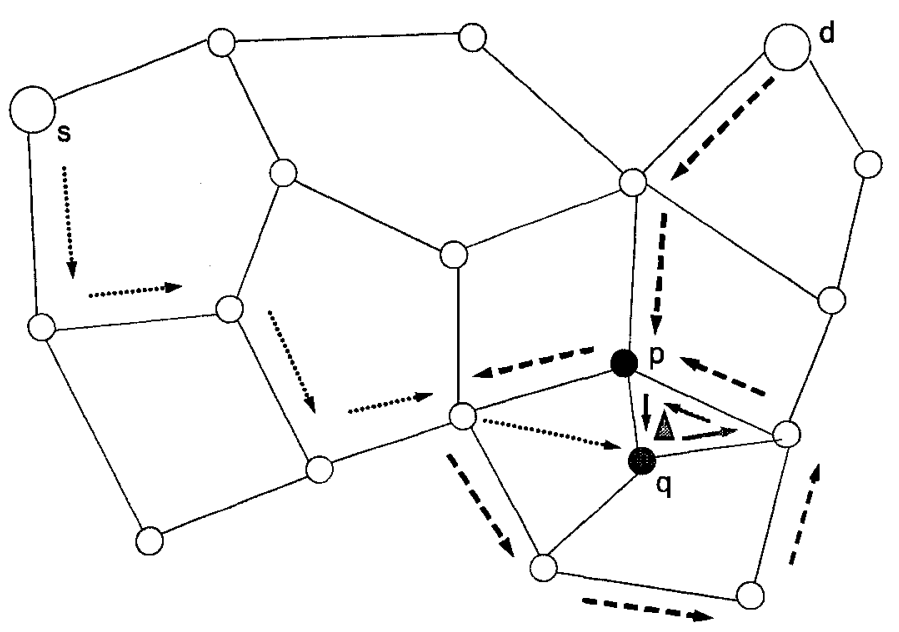

Figure 2.9: An illustration of GHT

pair (key, value), where key indicates the type of the event, and value is the sensor reading(s) about the event. When a sensor $u$ detects an event $E v t$, it hashes $E v t$ to a unique geographic location in the sensor field using the key of $E v t$. This implies that the same type of events will be hashed to the same location. Thereafter, $u$ sends a update message carrying the key-value pair of $E v t$ to the hash location through a greedy-face combine routing protocol GPSR (which is a duplicate of GFG [15]). By the property of the routing protocol, the update message will stop at a node closest to $E v t$ 's hash location. This node is called home node of $E v t$ (in fact all the events of the same type) and stores the data about $E v t$ retrieved from the update message.

The home node of an event type periodically sends a refresh message containing its stored relevant data along the home perimeter, the circle enclosing the hash location of the event type in the RNG graph used by the routing protocol. Each node in the home perimeter, called replica node, locally replicates the event data carried by the refresh message and appends any additional data it has to the message. To ensure a periodical refresh process to generate only local traffic, a node is assumed not to move many communication radio ranges in a period shorter than the refresh interval. If a refresh message reaches a node closer to the harsh location than the home node while traversing the home perimeter, this node will become new home node and then initiate its own refresh process. By this means, the home node is ensured 
to be a node closest to the hash location despite topology change. Both the home node and the replica nodes maintain an expire timeout for each event type to ensure data refreshness. They reset these timeouts upon receiving corresponding refresh messages, whether originated by themselves or not. The replica nodes in addition maintain a timeout according to which they initiate a similar refresh process to tolerate home node failure.

To improve performance on dynamic topology, a join optimization technique is used. That is, when a node $u$ detects a new neighbor $w$, it sends $w$ all the event entries it current has for which $w$ is closer to the event destination than $u$, and for which $u$ is the closest of its neighbors to that event destination. Besides, to balance storage load, structured replication can be employed. It is based on a grid hierarchy like the one used by GLS [44]. The home node of an event type selects a number of mirrors from the grid hierarchy in a certain depth $d$; a node that detects an event of such type stores the event at the nearest mirror of the home node. However, in this case, event queries must be routed to all the mirror nodes, leading to increased message complexity.

An execution example of GHT is shown in Figure 2.9. In this example, node $d$ sends a message containing event data of certain type $T$ towards the corresponding hash location and finally reaches the home node $p$. Node $p$ periodically sends refresh messages along the home perimeter of $T$ and finds a new comer $q$ yet-closer to the hash location of $T$. Then, $q$ becomes the new home node of $T$, and the home perimeter changes accordingly. A data consumer $s$ can obtain the data of type $T$ from either the home node or the replica nodes of $T$.

This scheme is a distributed implementation of centralized data storage. It is distributed in the sense that data of different types may be stored at different nodes. It has many disadvantages. Each refresh message has to carry all the relevant data, the amount of which can be as bad as the local memory size, stored by the initiator node. Every node needing certain type of data has to go to the home node of the data, even if it is close to the source of that type of data. If a type of data is generated and/or requested by a large number of nodes, the corresponding home node becomes communication bottleneck, and the network suffer from single point fail- 
ure. Although home node mirrors can be used to balance data storage and data insertion, the problem of frequent data query may become even worse since all the mirrors of a home agent must be accessed. Data aggregation of different types of data is not well supported.

\section{Landmark-based Information Storage and Retrieval}

The protocol [22] is based on a landmark-based routing protocol GLIDER [23]. GLIDER construct a voronoi diagram (LVC) over the network using landmark nodes as creating points. The boundary nodes of each voronoi cell (tile) is identified. The dual of the voronoi diagram, i.e., the Delaunay triangulation, is denoted by CDT. It compactly represents the topology feature of the underlying network. Every node locally stores CDT and uses it to constructs a shortest path tree rooted at its home landmark node. The idea of the protocol is to hash information to a certain tile (voronoi cell) and route the information to the hash tile through the shortest path in the shortest path tree rooted at the landmark node in the hash tile tile; on its way to the hash tile, the information is replicated in each intermediate tile. When a node wants query the information, it computes the hash tile using the same hash function, and sends a request along the shortest path to the hash tile in the shortest path tree rooted at the hash tile. During this process, it checks with every intermediate tile for the information. Because the storage path and request path are very likely to intersect, so the requester may get the information before it actually reaches the hash tile, reducing the probability of the root becoming a communication bottleneck and shortening the average query latency.

The key problems are how to store information in a tile and how to route within a tile. For the first problem, in a tile, a node is randomly selected, and then the protocol constructs a finger tree within the tile rooted at the selected node. The finger tree has three branches, one intersects the boundary towards the next tile in the path, the other two intersect the two adjacent boundaries. If the tile has less than three neighbor tiles, all the three intersection nodes will be on the boundary towards the the shortest path tree root. As for the second problem, the first node receives the request in the tile must resides on the boundary of the tile, 


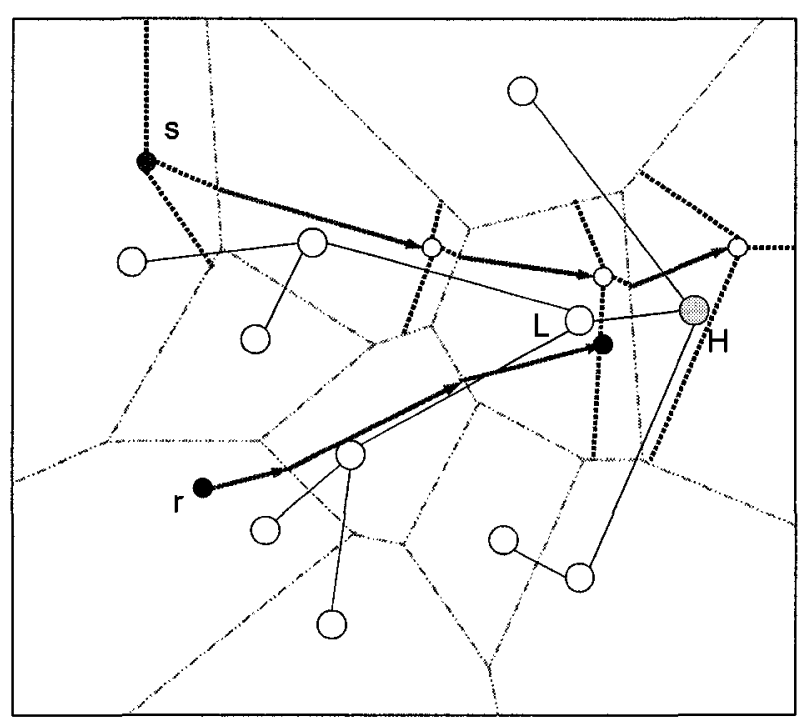

Figure 2.10: An illustration of Landmark

and it is proven that the entering boundary must not be adjacent to the exiting boundary. From the entering point, the request will be just greedily forwarded to the next tile, it is guaranteed the request will hit the finger tree and thus is able to retrieve the information. For the hash tile, its next tile is determined by the content of the information so that request can be guaranteed to intersect the finger tree.

Figure 2.10 shows such a shortest path tree rooted at landmark node $H$. Node $s$ hashes a piece of information to the tile of $H$ (this tile will be simply expressed by $H$ ) and routes the information to it. Along the route, a finger tree is built in each intermediate tile to store the information or data pointers. When node $r$ wants to obtain this information, it computes the hash tile $H$ using the same hash function and routes a query to $H$. In the figure, the query path meets the data storage path in the tile of landmark $L$ before reaching the hash tile $H$. The query path intersects the finger tree inside tile $L$, and the intersection point (node) will provides $r$ with the intended data.

In this storage scheme, an information consumer close to the producer may have to travel a long distance to obtain the information since they are possibly have a large distance to the root of the shortest path tree. And, for a pair of consumer and produce far apart from 
each other, the possibility of quick intersection of their update path and request path becomes low. And, since it is based on routing protocol GLIDER [23], it inherits all the drawbacks of GLIDER as follows: GLIDER relies on network-wide flooding for LVC construction and CDT distribution and requires a globalized lead election process for CDT computation, thus generating huge message overhead; GLIDER is vulnerable to the failure of the leader during CDT computation (re-electing a leader will bring even more message overhead); if a whole tile of nodes fail, the shortest path tree is disconnected, leading to routing failure.

\section{Double-ruling information brokerage}

Sarkar, Zhu and Gao [68] proposed a double-ruling information brokerage scheme. In this scheme, the sensors deployed in plane are mapped onto a virtual sphere of a pre-defined radius $r$ using stereographic projection, and the center of the sensor field is the south pole of the virtual sphere. Any point in the sensor field is mapped to a point $(x, y, z)$ on the sphere where $z \leq k$ for some $0<k<2 r$. By the properties of stereographic mapping, a diametral circle of the sphere is mapped to a circle in plane. The images of diametral circles of the sphere may have different size and centers, but they all encloses the south pole. Each sensor computes its image on the sphere and uses the image to execute the scheme as if it was on the sphere.

When an information producer wants to store the information in the network, it first finds the hash point of the information (by the information type) and then routes along a diametral circle, uniquely defined by the hash point and its own location, of the sphere to that point in both directions. All the intermediate nodes store either the complete information or a data pointer to where the data is stored in order to save storage space. Data of the same type from multiple producers is hashed to and aggregated at the same point on the sphere. When a data consumer $n$ wants to retrieve a certain type of data, it first computes the hash point $H$ using the data type and then retrieve data using different strategies as needed.

A simple strategy is that $n$ directly queries $H$ for the intended data through geographic 


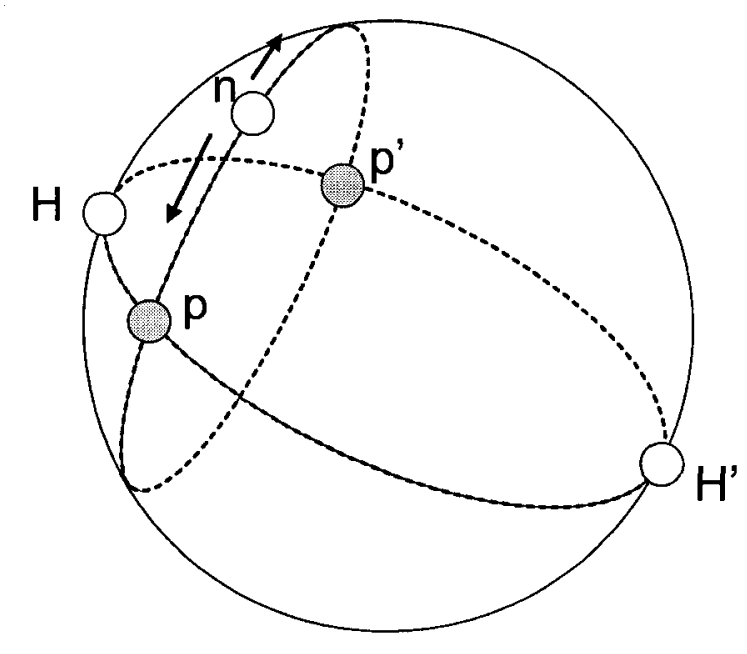

Figure 2.11: Distance-sensitive retrieval

routing. Another strategy is called distance-sensitive retrieval, in which $n$ travels along the circle with fixed distance to $H$ (possibly in both directions) to meet the replication curve of a data producer, as shown in Figure 2.11. A load-sensitive strategy is that $n$ travels any circle (not necessarily a diametral circle) that separates the hash point $H$ and the hash points' antipodal point $H^{\prime}$. Because this circle intersects all the replication circles of that data type, $n$ is able to aggregate all the relevant data by this strategy. The most advanced strategy is that $n$ obtains all the information stored in the network by traveling along an arbitrarily picked diametral circle.

This scheme is a special case of the quorum-based location service [48,72]. It provides more flexibility in data retrieval and better load balancing in data storage than generic one. Nevertheless, it generates bottleneck problem in the network, especially when some types of data are continuously generated, because the same type of data is always routed to the same hash node. Compared with generic quorum-based scheme, this scheme also often generates relatively long update and search routes when the service is available nearby. 


\section{CHAPTER 3 FOCUSED COVERAGE BY MOBILE SENSORS I}

\section{E}

xisting sensor self-deployment algorithms emphasize only on coverage formation over a Region of Interest (ROI). In this chapter, we however address a new sensor selfdeployment problem, focused coverage formation around a Point of Interest (POI), and define a corresponding evaluation metric, coverage radius. We propose two solution protocols, Greedy Advance (GA) and Greedy-Rotation-Greedy (GRG), which are to our knowledge the first sensor self-deployment algorithms that operate in a strictly localized manner and are yet able to provide coverage guarantee on size maximization and hole absence. Furthermore, we show that GRG guarantees optimal (i.e., maximized) hexagonal coverage radius and optimal or near optimal circular coverage radius.

In Section 3.1, we introduce the concepts of focused coverage and coverage radius, and briefly describe the working principle of GA and GRG; in Section 3.2, we define the network model; in Section 3.3, we introduce a planar graph, equilateral triangle tessellation (TT), to be used by GA and GRG; in Section 3.4, we present the details of the two algorithms; in Section 3.5, we prove their correctness and analyze their coverage radius property; in Section 3.6, we evaluate the performance of GA and GRG on convergence time, energy consumption and node collision through extensive simulation; in Section 3.7, we summarize the chapter. 


\subsection{Introduction}

There exist a class of sensor applications, where sensors are designated to monitor concerned events or environmental changes happening around a strategic site, called Point of Interest (POI). For instance, in a battle field scenario, sensors are deployed around a battalion headquarter to timely detect intrusion events, whose distance to the headquarter reflects their degree of danger. Another example are sensors scattered around a chemical plant to monitor its distance-dependent pollutional impact on the soil/air in the vicinity. These applications uniquely require that an area close to the POI have higher priority to be covered than a relatively distant area. We call the coverage of such a surrounding network focused coverage.

Size and hole are the key evaluation metrics for traditional area coverage. They reflect the sensitivity of a sensor network over a ROI. An ideal area coverage has maximized size and zero sensing hole. However, in the focused coverage case with a POI, measuring size and hole existence is no longer sufficient for evaluation purpose, because distance from the POI to uncovered areas also makes significant sense and must be taken into consideration. Under this circumstance, we introduce an additional metric, coverage radius.

Definition 3.1. For a focused coverage, its coverage radius is defined as the radius of its Essential Coverage Disc (ECD), which is the maximal hole-free disc enclosed by sensors in the coverage region and centered at the POI.

In continuous domain, there may exist a sensor node at every point in the coverage region, and the ECD therefore has a circular shape, as shown in Figure 3.1. In this case, coverage radius is called circular radius and measured by Euclidean distance. In discrete domain, the shape of the ECD is however not circular but polygonal, and coverage radius is thus correspondingly referred to as polygonal radius and alternatively measured by layer distance. Layer distance, also called convex layers in computational geometry or Tukey's depth in statistics, represents the number of successive complete convex polygons adjacently surrounding the POI. More precisely, we consider a discrete set of convex polygons $\mathcal{P}_{i}(i=1,2, \ldots)$ that are 


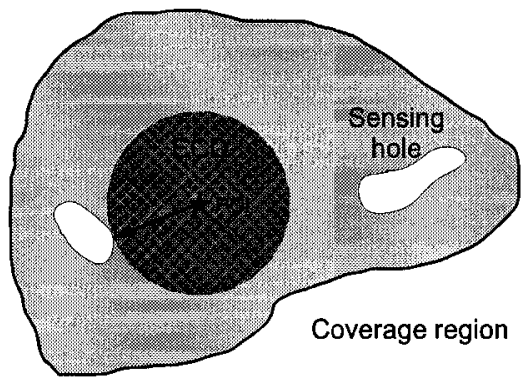

(a) Restriction from hole

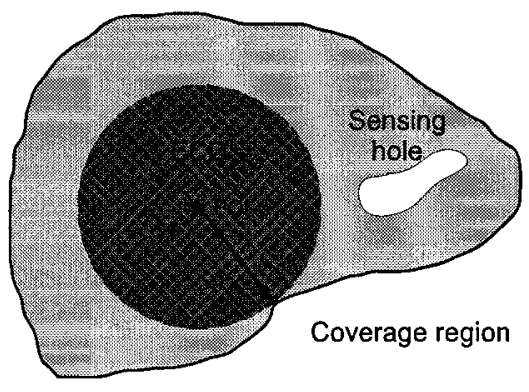

(b) Restriction from border

Figure 3.1: Circular coverage radius

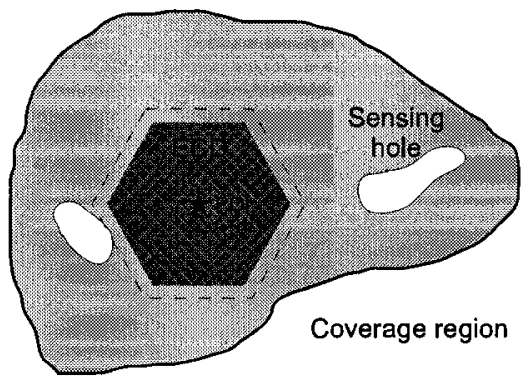

(a) Restriction from hole

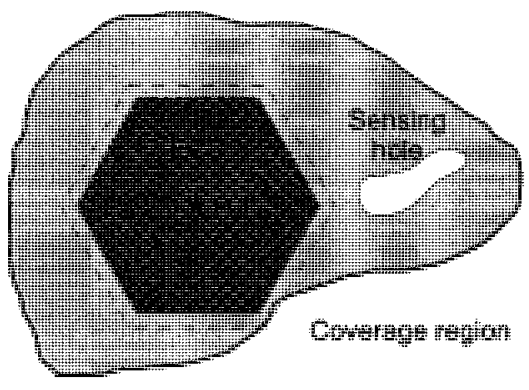

(b) Restriction from border

Figure 3.2: Polygonal (hexagonal) coverage radius

composed of sensors, centered at the POI and having a diameter of $i * d$ for some constant $d$. We count the total number of such polygons lying completely in the sensor network's coverage region. Figure 3.2 illustrates the definition of polygonal (hexagonal) coverage radius. The hexagonal radius is 6 in Figure 3.2(a) and 8 in 3.2(b). Whichever measurement is used, coverage radius should be maximized.

\subsubsection{Our contributions}

We pinpoint a new sensor self-deployment problem, i.e., focused coverage formation around a Point of Interest (POI), and introduce an evaluation metric, i.e., coverage radius, which reflects the significance of distance from the POI to uncovered areas. We propose two strictly localized solution protocols, Greedy Advance (GA) and Greedy-Rotation-Greedy (GRG), which are the first localized sensor self-deployment algorithms that provide coverage 
guarantee on size maximization and hole absence.

GA and GRG convert the area coverage problem to a vertex coverage problem over a locally-computable equilateral triangle tessellation (TT). In the two algorithms, self-governing sensors relocate themselves on the TT grid and move from vertex to vertex to surround the POI, according to their one-hop neighborhood information only. Specifically, in GA, nodes greedily proceed as close to the POI as they can; in GRG, when their greedy advance is blocked, nodes rotate around the POI along a hexagon defined by TT edges to a TT vertex where greedy advance can resume. In both algorithms, when sensors are compactly placed or collide, they may move away from the POI.

Both GA and GRG yield a connected network of TT layout with no sensing hole. GRG in addition assures a hexagon coverage shape centered at the POI. Thanks to their purely localized nature, the two algorithms are resilient to node failures and work regardless of network disconnectivity. We prove their correctness and analyze their coverage radius property. Our analysis shows that GRG guarantees optimal hexagonal coverage radius, and optimal or near optimal (with a factor in $[0.88,1]$ ) circular coverage radius. We evaluate their performance on convergence time, energy consumption and node collision through extensive simulation.

\subsection{Model and Definitions}

We consider an asynchronous MSN of unknown size $n$, which is randomly dropped in a two-dimensional free field (e.g., an area on ocean surface in practice) and may possibly be disconnected at initiation. Sensors bear the same communication radius $r_{c}$ and the same sensing radius $r_{s}$; they know about the location of a Point of Interest (POI), denoted by $\Pi$. Because the global coordination system can be easily (through trivial local processing) converted to one with $\Pi$ as origin, we use $(0,0)$ as $\Pi$ without loss of generality.

The goal is to develop a strictly localized sensor self-deployment algorithm that yields a network surrounding $\Pi$ with an equilateral triangle tessellation (TT) layout. The reasons 
Table 3.1: Notations

\begin{tabular}{|c|c|}
\hline$\%$ & Modulus operation \\
\hline$\Pi$ & A given Point of Interest (POI) \\
\hline$n$ & Network size; a unknown value \\
\hline$G_{T T}$ & A graph of equilateral triangle tessellation (TT) \\
\hline$l_{e}$ & The edge length of $G_{T T}$; equal to $\sqrt{3} r_{s}$ \\
\hline $\operatorname{Path}_{S}(u, v)$ & A shortest path connecting two vertices $u$ and $v$ in $G_{T T}$ \\
\hline $\operatorname{Dist}_{T T}(u, v)$ & The TT distance of $u$ and $v$; defined as the number of edges in Path $_{S}(u, v)$ \\
\hline $\mathcal{H}_{i}$ & A hexagon composed of vertices $v$ in $G_{T T}$ such that $\operatorname{Dist}_{T T}(v, \Pi)=i$ \\
\hline$C_{i}$ & The inscribed disc of $\mathcal{H}_{i}$ \\
\hline $\mathcal{R}_{j}$ & A ray defined by $\mathcal{H}$ hexagon corner vertices \\
\hline $\mathcal{S}_{j}$ & The sector defined by $\mathcal{R}_{j}$ (inclusive) and $\mathcal{R}_{(j+1) \% 6}$ \\
\hline$\langle i, j, k\rangle$ & The address of the vertex of in-sector index $k$ in $\mathcal{S}_{j}$ on $\mathcal{H}_{i}$ \\
\hline $\operatorname{Coord}(i, j, k)$ & The coordinate of vertex at address $\langle i, j, k\rangle$ \\
\hline$v(i)$ & The total number of vertices enclosed by $\mathcal{H}_{i}$ inclusive \\
\hline$v^{-1} 0$ & The inverse function of $v 0$ \\
\hline$\gamma_{o p t}^{H}$ & Optimal hexagonal coverage radius \\
\hline$\gamma^{H}$ & Actual hexagonal coverage radius \\
\hline$\gamma_{o p t}^{C}$ & Optimal circular coverage radius \\
\hline$\gamma^{C}$ & Actual circular coverage radius \\
\hline$\kappa$ & The outmost hexagon of a focused coverage \\
\hline$\kappa^{\prime}$ & The outmost fully-occupied hexagon of a focused coverage \\
\hline$\rho\left(\kappa^{\prime}\right)$ & The number of nodes contributing to redundant coverage \\
\hline$\mu\left(\kappa^{\prime}\right)$ & The number of nodes constituting the essential coverage disc (ECD) \\
\hline
\end{tabular}

why this TT layout is required are that it maximizes the coverage area of a given number of nodes without coverage gap when nodal separation is equal to $\sqrt{3} r_{s}[11,52,87]$, and that it automatically maintains network connectivity when $r_{c} \geq \sqrt{3} r_{s}$. As an additional requirement, the final network should have maximized coverage radius with respect to $\Pi$.

We consider this sensor self-deployment problem under the following commonly-used assumptions: (1) $r_{c} \geq \sqrt{3} r_{s}$; (2) sensors are aware of their own geographic location, which is represented by an $(x, y)$ coordinate, by a localization system like GPS; (3) through lower-layer protocols (minor modification may apply), sensors have the information about their one-hop neighbors, i.e., location, status (still or moving) and destination (if moving). Table 3.1 lists the main notations to be used in the rest of this chapter. 


\subsection{Equilateral Triangle Tessellation}

An equilateral triangle tessellation (TT) is a planar graph that is composed of congruent equilateral triangles. With a common orientation (e.g., north) and a pre-determined edge length $l_{e}$, each sensor is able to locally compute a unique TT with $\Pi$ as vertex. Denote the TT graph by $G_{T T}$. In our work, $l_{e}$ is set to $\sqrt{3} r_{s}$. It is because we want to finally locate sensors on vertices of $G_{T T}$, and this particular edge length ensures connectivity and minimizes sensing range overlapping $[11,52,87]$.

Let $\operatorname{Path}_{S}(u, v)$ represent a shortest path connecting two vertices $u$ and $v$ in $G_{T T}$. The $T T$ distance between $u$ and $v$ is defined as the number of edges in $\operatorname{Path}_{S}(u, v)$, and denoted by $\operatorname{Dist}_{T T}(u, v)$. In $G_{T T}$, there are $6 i$ many vertices with equal TT distance $i$ to $\Pi$. These vertices together constitute a distance- $i$ hexagon, denoted by $\mathcal{H}_{i} . \mathcal{H}$ hexagons are concentric to $\Pi$. Let $v(i)$ be the total number of vertices enclosed by $\mathcal{H}_{i}$, inclusive. Then

$$
v(i)=1+\sum_{q=1}^{i}(6 q)=3 i(i+1)+1
$$

We call the vertices located at hexagon corners corner vertices, the others edge vertices. Corner vertices form 6 rays jointing at $\Pi$ with mutual angle of $\frac{\pi}{3}$ and divide the entire plane evenly into 6 sectors. The sector toward south is called "Sector 0" and denoted by $\mathcal{S}_{0}$; the other sectors are named after their sequence number after $\mathcal{S}_{0}$ in counterclockwise direction.

For $0 \leq j<6, \mathcal{S}_{j}$ is defined by two neighboring rays $\mathcal{R}_{j}$ and $\mathcal{R}_{(j+1) \% 6}$, where $\%$ stands for modulus operation; its clockwise next sector and counterclockwise next sector are $\mathcal{S}_{(j+5) \% 6}$ and $\mathcal{S}_{(j+1) \% 6}$, respectively. A partial $G_{T T}$ is drawn in Figure 3.3, where sectors and rays are labeled, and $\mathcal{H}$ hexagons are highlighted.

We assign every vertex $v$ on $\mathcal{H}_{i}$ an in-hexagon index and an in-sector index. The former, denoted by $k^{\prime}\left(0 \leq k^{\prime}<6 i\right)$, is equal to the number of TT edges from $v$ to $\mathcal{R}_{0}$ along $\mathcal{H}_{i}$ in the clockwise direction, while the latter, referred to as $k(0 \leq k<i)$, is equal to the number of TT edges from $v$ to $\mathcal{R}_{j}$ along $\mathcal{H}_{i}$ in the clockwise direction within its residing sector $\mathcal{S}_{j}$. Notice 


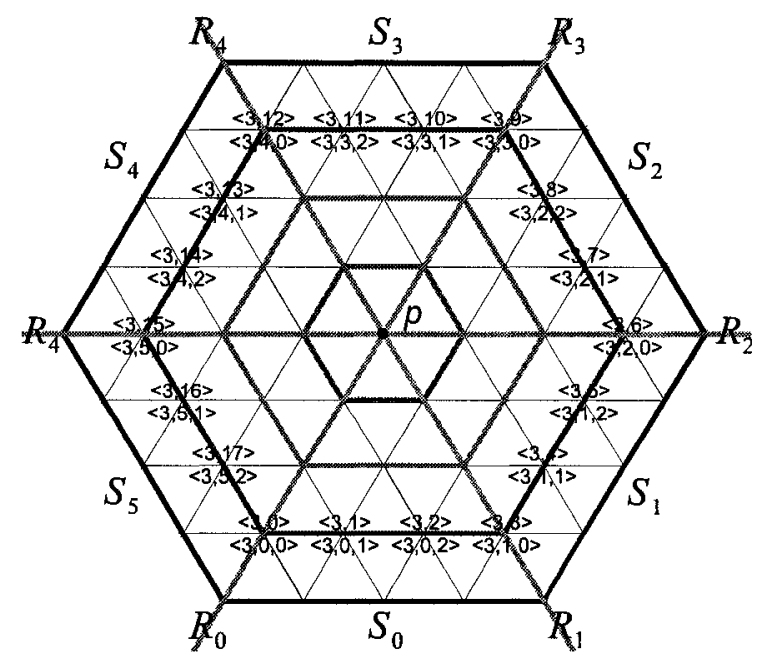

Figure 3.3: An partial equilateral triangle tessellation

that $k^{\prime} \% i=0$ and $k=0$ if $v$ is corner vertex. We can uniquely address $v$ using either a pair $\left\langle i, k^{\prime}\right\rangle$ or a triple $\langle i, j, k\rangle$, which are mutually convertible by $k^{\prime}=i j+k, j=\left\lfloor\frac{k^{\prime}}{i}\right\rfloor$ and $k=k^{\prime} \% i$. Figure 3.3, displays the addresses of vertices of $\mathcal{H}_{3}$ in both formats.

In order to be clearly differentiated from geographic coordinate, a vertex's address will be expressed in the format of $\langle i, j, k\rangle$ in the sequel. For consistency, we consider $\Pi$ as a special hexagon $\mathcal{H}_{0}$, and define its address as $\langle 0, *, 0\rangle$, where $*$ may be any non-negative integer less than 6 as needed. Denote the geographic coordinate of $\langle i, j, k\rangle$ by $\operatorname{Coord}(i, j, k)$. Observe that $\langle i, j, k\rangle$ can be obtained by counterclockwise rotating $\langle i, 0, k\rangle$ a degree of $\frac{\pi}{3} i$ about $\Pi$. Recall that $\Pi$ is at $(0,0)$. Because $\operatorname{Coord}(i, 0, k)=\left(\left(k-\frac{1}{2} i\right) l_{e},-\frac{\sqrt{3}}{2} i l_{e}\right)$, where $l_{e}=\sqrt{3} r_{s}$ is the edge length of $G_{T T}$, we have

$$
\begin{aligned}
\operatorname{Coord}(i, j, k)= & \left(\left(\left(k-\frac{1}{2} i\right) \cos \left(\frac{\pi}{3} j\right)+\frac{\sqrt{3}}{2} i \sin \left(\frac{\pi}{3} j\right)\right) l_{e}\right. \\
& \left.\left(\left(k-\frac{1}{2} i\right) \sin \left(\frac{\pi}{3} j\right)-\frac{\sqrt{3}}{2} i \cos \left(\frac{\pi}{3} j\right)\right) l_{e}\right)
\end{aligned}
$$




\subsection{Protocol Description}

Recall our goal defined in Section 3.2. If we deploy sensors at the vertices around $\Pi$ in the TT graph $G_{T T}$ introduced in Section 3.3, we automatically get a network with required TT layout; if we further carefully control the coverage shape and assure the absence of empty TT vertices within the coverage region, we may as well achieve the desired focused coverage with no sensing hole and with (near) maximized radius. By this means, we actually convert the area coverage problem to a vertex coverage problem in $G_{T T}$.

Based on the above intuition, we propose two strictly localized sensor self-deployment algorithms, Greedy Advance (GA) and Greedy-Rotation-Greedy (GRG). In the two algorithms, sensors do not necessarily store entire $G_{T T}$ in their memory. It is sufficient for them only to compute their neighboring TT vertices at runtime.

For simplicity, a TT vertex $v$ is said to be occupied by a node if the node is not moving and is located in close proximity to $v$, or if the node is moving toward $v$ even if it is far from $v$; $\Pi$ is also considered occupied in the case that it is not physically occupiable. We assume for the time being that all the sensors are initially located at distinct vertices of $G_{T T}$. This temporary assumption will be relaxed immediately after, in Section 3.4.4.

\subsubsection{Greedy Advance (GA)}

In GA, a node moves greedily along TT edges as close to $\Pi$ in terms of TT distance as it can. For every step of its greedy advance, a node may have one or two options, depending on its residing vertex. Precisely speaking, it has one and only one possible next hop $\langle i-1, j, k\rangle$ if its residing vertex $\langle i, j, k\rangle$ is a corner vertex (i.e., $k=0$ ), or two possible next hops $\langle i-1, j, k-1\rangle$ and $\langle i-1, j, k\rangle$ if, otherwise, it is an edge vertex (i.e., $0<k<i$ ). Figure 3.4 shows six nodes and their possible next hops marked by thick arrowed lines. Each node chooses from its next hop candidates one that will not cause node collision according to its best local knowledge. If no such a next hop is available, it stays still. A still node resumes 


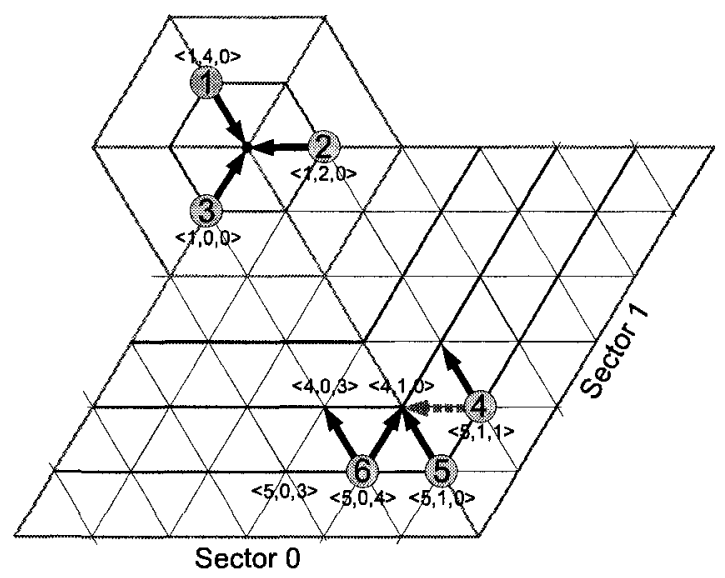

Figure 3.4: Hop selection in GA

its greedy advance whenever possible.

Randomly pick an edge vertex, say $\langle 4,0,3\rangle$. It has two possible previous hops $\langle 5,0,3\rangle$ and $\langle 5,0,4\rangle$. Examine the example scenario given in Figure 3.4. If $\langle 4,0,3\rangle$ is chosen as next hop by node 6 and another node (which is not shown in the figure) at $\langle 5,0,3\rangle$ at the same time, node collision will occur. However, since the two nodes are neighboring each other, they know about the potential collision and thus can prevent it from happening by the following rule:

Rule 3.1 (Priority Rule). If two nodes are trying to greedily move to $\langle i, j, k\rangle$ respectively from $\langle i+1, j, k\rangle$ and $\langle i+1, j, k+1\rangle($ or $\langle i+1,(j+5) \% 6, i\rangle$ if $k=0)$, the one from $\langle i+1, j, k+1\rangle$ (resp., $\langle i+1, j, k\rangle)$ has higher priority than the other.

Special attention should be paid to any corner vertex $\langle i, j, 0\rangle$ that has totally three possible previous hops $\langle i+1,(j+5) \% 6, i\rangle,\langle i+1, j, 0\rangle$ and $\langle i+1, j, 1\rangle$. Let us again examine the scenario in Figure 3.4. By the priority rule, neither nodes 4 and 5 nor nodes 5 and 6 may collide at corner vertex $\langle 4,1,0\rangle$. But nodes 4 and 6 will, if they simultaneously move to $\langle 4,1,0\rangle$, and the collision is not locally avoidable since the two nodes are not aware of each other. To eliminate this undesired situation, we can simply force node 4 not to take $\langle 4,1,0\rangle$ as next hop by the following rule:

Rule 3.2 (Forbiddance Rule). A node located at $\langle i+1, j, 1\rangle$ does not chose $\langle i, j, 0\rangle$ as greedy next hop. 


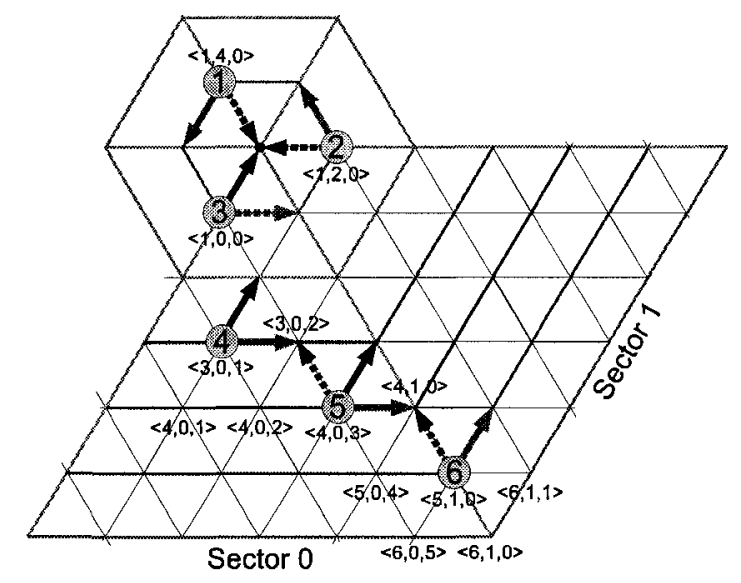

Figure 3.5: Hop selection in GRG

In Figure 3.4, hop selection that is forbidden by the forbiddance rule is shown by dashed arrowed lines.

$\Pi$ has six possible previous hops, i.e., the six vertices in $\mathcal{H}_{1}$, in total. Consider a scenario where six nodes are occupying $\mathcal{H}_{1}$ vertices, and $\Pi$ is not occupied. In this particular case, a deadlock occurs due to the priority rule, and none of the six nodes will attempt to move to $\Pi$, resulting a sensing hole at $\Pi$. To avoid this deadlock, we define an additional rule as follows:

Rule 3.3 (Innermost-Layer Rule). A node located at $\langle 1, j, 0\rangle$ moves to $\Pi$ as long as $\Pi$ is not occupied.

The innermost-layer rule may cause node collision at $\Pi$. However, such a collision takes place at most once, because a node will stay at $\Pi$ after it reaches $\Pi$, and because no node will try to move to $\Pi$ once $\Pi$ is occupied. Later, in Sec. 3.4.4, we will discuss how to resolve node collision.

\subsubsection{Greedy-Rotation-Greedy (GRG)}

GRG involves not only greedy advance but also a new type of node movement - rotation, which forms the final network in a shape of hexagon centered at $\Pi$. An arbitrary node, when its greedy advance is blocked, tries rotation around $\Pi$ along its residing hexagon without increasing its TT distance to $\Pi$. Note that rotation should be restricted to a particular, 
say counterclockwise, direction so as to avoid unnecessary collisions between rotating nodes. Formally speaking, a node at $\langle i, j, k\rangle$ chooses only $\langle i, j, k+1\rangle($ or $\langle i,(j+1) \% 6,0\rangle$ if $k=i-1)$ as rotation next hop. Figure 3.5 shows six nodes and their possible next hops, among which rotation next hops and greedy next hops are differentiated using different colors. A node stops rotating when it reaches a vertex where greedy advance can resume, or when it returns to the vertex where it starts rotating. A node that rotates back to its rotation starting point is called return node. To properly react to its neighborhood change (due to sensor deployment or node failures), a return node resets its rotation starting point to nil whenever it observes the status change of its rotation next hop from unoccupied to occupied.

In an asynchronous environment, a rotating node on $\mathcal{H}_{i}$ may never be able to move onto $\mathcal{H}_{i-1}$ despite the vacancies on $\mathcal{H}_{i-1}$, if its neighboring nodes on $\mathcal{H}_{i-1}$ rotate together with it and keep blocking its greedy advance. To prevent this problematic situation, we define the following rule:

Rule 3.4 (Suspension Rule). A node located on $\mathcal{H}_{i-1}$, before starting its next rotation step, checks if there is any neighbor rotating on $\mathcal{H}_{i}$. If yes, it gives up its rotation plan.

Consider node 4 in Figure 3.5. Suppose that its greedy advance is blocked, that $\langle 3,0,0\rangle$ and $\langle 4,0,1\rangle$ are both occupied, and that $\langle 3,0,2\rangle$ and $\langle 4,0,2\rangle$ are both empty. According to the suspension rule, node 4 does not rotate to $\langle 3,0,2\rangle$ in this case. The intuition is that node 4 knows that, if it itself stays put, the node at $\langle 4,0,1\rangle$ will rotate to $\langle 4,0,2\rangle$ and then greedily advance to $\langle 3,0,2\rangle$. By the suspension rule, a rotating $\mathcal{H}_{i}$ node will either meet an empty vertex on $\mathcal{H}_{i-1}$, surpassing some $\mathcal{H}_{i-1}$ nodes in between, or find no vacancy on $\mathcal{H}_{i-1}$ and stops at its rotation starting point.

Rule 3.5 (Competition Rule). In the case that a greedily advancing node and a rotating node are targeting at the same vertex, the former proceeds as usual, while the latter changes its deployment decision accordingly.

In GRG, each non-POI vertex $\langle i, j, k\rangle$ has two possible previous hops, $\langle i+1, j, k\rangle$ and $\langle i+1, j, k+1\rangle$ (or, $\langle i+1,(j+5) \% 6, i\rangle$ if $k=0$ ), for greedy advance and one previous hop 
$\langle i, j, k-1\rangle$ (resp., $\langle i,(j+5) \% 6, i-1\rangle)$ for rotation movement. As discussed in Section 3.4.1, greedy collision does not happen at $\langle i, j, k\rangle$, because the two greedy previous hops are neighboring each other. Observe that, vertices $\langle i+1, j, k\rangle$ and $\langle i, j, k-1\rangle$ (or, $\langle i+1,(j+5) \% 6, i\rangle$ and $\langle i,(j+5) \% 6, i-1\rangle$ if $k=0)$ are also each other's neighbor. Therefore, the greedy-rotation collision caused by nodes from these two vertices can be locally avoided as well, by the competition rule. Now, let us examine the greedy-rotation collision due to nodes from $\langle i+1, j, k+1\rangle$ and $\langle i, j, k-1\rangle$ (or, $\langle i+1, j, k\rangle$ and $\langle i,(j+5) \% 6, i-1\rangle$ if $k=0)$. Because the two nodes are out of each other's communication range, the collision can not be inferred by them from their local knowledge. Depending on the way of handling this situation, GRG has two variants: Collision alloWance (CW) and Collision aVoidance (CV).

\section{GRG-CW}

In this variant, no additional restriction is applied; greedy-rotation collision is allowed. During a greedy-rotation collision, the rotating node is required to make its next deployment decision first, which immediately affects the other's motion plan. The reason why the rotating node is given priority is to prevent collision loop caused by endless rotating-retreating role switch. We will come back to this in Section 3.4.4, when introducing retreat movement for collision resolution.

By the ordered sequence of decision making, greedy-rotation collision could appear as a transient phenomenon. For example, in the scenario in Figure 3.6(b), collision between nodes 2 and 6 takes place at $d$ and is then automatically resolved. However, there is no assurance that collision does not remain permanently (this drawback will be resolved later, in Section 3.4.4).

\section{GRG-CV}

In this variant, greedy-rotation collision becomes impossible due to the edge rule and the corner rule to be introduced below. The objective of the two rules is to restrict greedy advance to rotation direction, i.e., counterclockwise direction. The intuition stems from the observation 
that locally-unknown greedy-rotation collision takes place only when greedy advance and rotation are opposite to each other. For example, in Figure 3.5, node 4 and 5 have a potential collision at $\langle 3,0,2\rangle$; node 5 and 6 have a potential collision at $\langle 4,1,0\rangle$.

Rule 3.6 (Edge Rule). A node located at edge vertex $\langle i, j, k\rangle$ only considers $\langle i-1, j, k\rangle$ (or $\langle i-1,(j+1) \% 6,0\rangle$ if $k=i-1)$ as the next hop of its greedy advance.

Rule 3.7 (Corner Rule). A node located at corner vertex $\langle i, j, 0\rangle$ performs no greedy advance.

Special attention should be paid to the nodes located on $\mathcal{H}_{1}$. Because of the corner rule, none of these nodes will move to $\Pi$, generating a sensing hole at $\Pi$. Under this circumstance, we define a particular vertex, denoted by $\operatorname{Gate}(\Pi)$, on $\mathcal{H}_{1}$ as the gateway to $\Pi$ and allow only a gateway node to move to $\Pi$. More formally, we define

Rule 3.8 (Gateway Rule). A node located at Gate(П) conducts no rotation but greedy advance.

By the corner rule and the gateway rule, any $\mathcal{H}_{1}$ node not located at Gate( $\left.\Pi\right)$ has to first rotate to Gate( $\Pi)$ in order to occupy $\Pi$. Hence, a gateway node's greedy advance is safe as no other $\mathcal{H}_{1}$ node is moving to Gate $(\Pi)$ in the mean time.

Figure 3.5, where Gate $(\Pi)=\langle 1,0,0\rangle$, shows the possible next hops of 6 nodes in GRG-CV with solid arrowed lines. In the figure, dashed arrowed lines imply the hop selection allowed in GRG-CW but forbidden by in GRG-CV. Thanks to the strict hop selection rules of GRG$\mathrm{CV}$, any potential collision is been aware of by the colliding nodes and is therefore locally inhibitable.

\subsubsection{Execution examples}

In the following, we shall comparatively show how GA and the two variants of GRG, i.e., GRG-CW and GRG-CV, work through examples. Although the two algorithms operate regardless of network size and asynchrony, we consider for easy of understanding a simple 


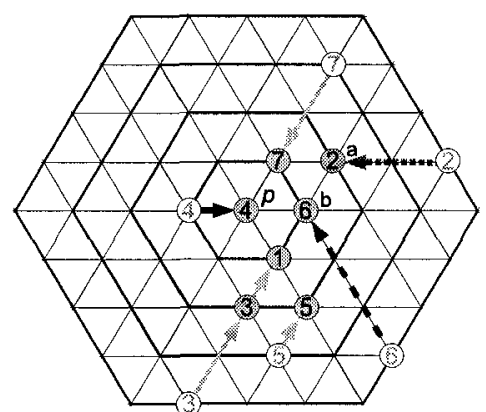

(a) GA

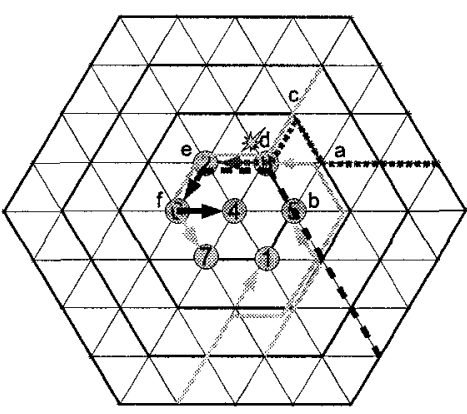

(b) GRG-CW

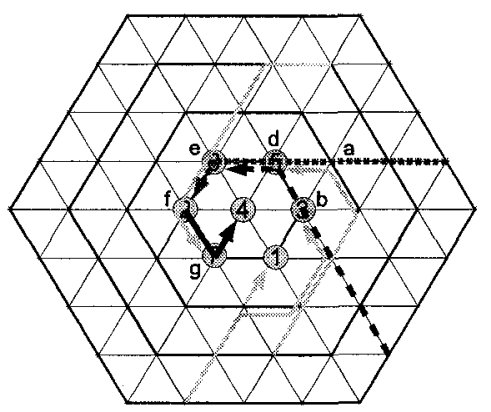

(c) GRG-CV

Figure 3.6: Final node distribution after sensor self-deployment

scenario, where 7 nodes initially placed at distinct TT vertices start the self-deployment algorithms simultaneously and move at the same speed. Figure 3.6 shows the final node distribution obtained respectively by GA, GRG-CW and GRG-CV. In the figure, node trajectories are marked by thick arrowed lines, pointing from initial position to final position. Note that the initial position of node 1 is the final position of node 3 in Figure 3.6(a), and that the initial position of node 4 is the final position of node 6 in Figure 3.6(b) and of node 2 in Figure 3.6(c). To have a clear view of node movement, we focus only on the three nodes 2,4 and 6 .

Figure 3.6(a) indicates that, when GA is applied, the three nodes 2, 4 and 6 move toward $\Pi$ and stop respectively at $a, \Pi$ and $b$ according to the forbiddance rule and the innermostlayer rule. As shown in Figure 3.6(b), when GRG-CW is employed, node 4 proceeds in the same way as in GA; whereas, nodes 2 and nodes 6 travel along an extended path. Specifically, after reaching $a$, node 2 finds that vertex $d$ is occupied by node 7 , and that greedy advance to $b$ is forbidden by the forbiddance rule. Under this circumstance, it has to rotate around $\Pi$ along its residing hexagon. When node 2 rotates to $c$, node 6 arrives at $b$. At that moment, $d$ becomes empty due to node 7's leaving, and the POI has been taken by node 4 . Then node 2 decides to greedily proceed to $d$, and node 6 also decides to rotate to $d$. Because the two nodes are not neighboring each other, they do not know each other's motion plan and consequently collide at $d$. Because a rotating node is given priority to take the next deployment step in such a greedy-rotation collision (see Section 3.4.2), node 6 continues its rotation, while node 2 has 
to wait. Finally, node 6 rotates to its final position $f$, passing by $e$; node 2 rotates to $e$ after node 6 leaves $e$ for $f$.

Observe Figure 3.6(c), which corresponds to GRG-CV. Because node 4 is not allowed to directly move to $\Pi$ by the corner rule, it has to first rotate to a particular gateway vertex (which is set to be $g$ in this example) and then greedily moves to $\Pi$ by the gateway rule. Node 7 can not start with greedy advance but has to perform rotation first according to the corner rule, ending up with a completely different trajectory, which directly affects node 2's deployment process: now, after reaching $a$, node 2 is able to continue its greedy advance and immediately proceed to $d$ since no one is occupying $d$. When node 6 reaches $b$ through greedy advance, node 2 just arrives at $d$, and node 4 already got to $\Pi$. Then node 6 has to wait, since its can perform neither greedy advance or rotation in this case. The suspension of node 6's movement in turn affects nodes 3 and 5's trajectory, which we will however not go through here. Finally, node 2 rotates to $f$, and node 6 rotates to $e$. Notice that the collision between node 2 and 6 in GRG-CW does not occur.

\subsubsection{Resolving node collision}

We previously assumed that nodes are initially located at distinct TT vertices, which however rarely happens in practice because of randomized node distribution. This temporary assumption can be readily relaxed by the following rule:

Rule 3.9 (Alignment Rule). A node located inside or on the border of a TT triangle $\Delta v_{1} v_{2} v_{3}$ moves to the triangle vertex $v_{t}$ that is occupied by the least number of nodes. If more than one such triangle vertex exists, the closest is selected. In case of ties, a random choice is made.

The alignment rule is very likely to cause various node collisions. In the following, we are going to introduce a new type of movement - retreat for collision resolution. Retreat movement is the opposite of greedy advance. It happens from a vertex of hexagon $\mathcal{H}_{i}(i \geq 0)$ to a vertex of hexagon $\mathcal{H}_{i+1}$. With the help from retreat movement, permanent collision no longer remains, and both GA and GRG gain the ability to spread out compact networks. 


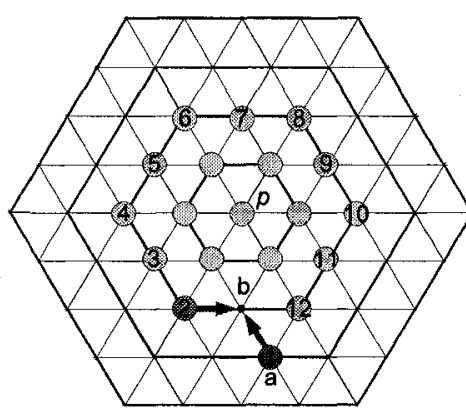

(a) Rotation

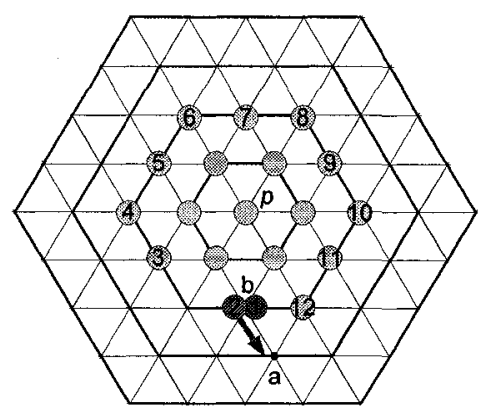

(b) Rotating-retreating role switch

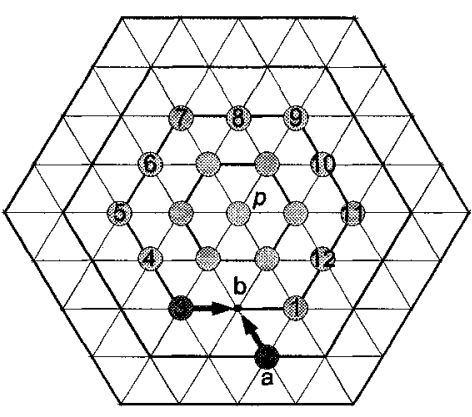

(c) Greedy advance

Figure 3.7: An example of collision loop in GRG-CW

After some nodes collide at a TT vertex, they enter a local ranking process. These colliding nodes are able to do the ranking locally and independently because they are neighboring each other. During this process, each of them is assigned a rank based on either a random selection or certain criterion (if available) such as residual energy or node ID or the combination thereof. Then, the node with the highest rank makes its next deployment decision first, and the others follow in accordance with the decreasing order of their ranks. If the $t$-th node decides to stay, every node with rank lower than $t$ retreats by the following rule:

Rule 3.10 (Retreat Rule). When a node located at a $\mathcal{H}_{i}$ vertex decides to retreat, it retreats to one of its neighboring $\mathcal{H}_{i+1}$ vertices that is occupied by the least number of nodes. In case of ties, a random choice is made.

In GRG-CW, retreat movement might cause greedy-rotation collision loop and consequently endless movement in some rare scenarios like the one given in Figure 3.7. Figure 3.7(a) shows that $\Pi$ and $\mathcal{H}_{1}$ vertices have been occupied, and that there is only one empty vertex $b$ on $\mathcal{H}_{2}$. Under this situation, node 1 located at vertex $a$ on $\mathcal{H}_{3}$ decides to greedily moving to $b$, and node 2 meanwhile decides to rotate to $b$. Because the two nodes 1 and 2 are not neighboring each other, they will collide at $b$ as shown in Figure 3.7(b). Assume that, after collision, node 1 is assigned a higher rank than node 2 . In this case, node 1 decides to stay at $b$, while node 2 has to retreat to $\mathcal{H}_{k+1}$ by the retreat rule. Suppose that node 2 happens to retreat to $a$ and that node 1 meanwhile finishes one rotation step. We end up with a scenario 
in Figure 3.7(c) that is exactly the same as the one in Figure 3.7(a).

Since we are in an asynchronous environment and we do not assume any specific ranking method, it is possible that similar thing happens when node 2 makes its greedy advance later on. If continuing that way, each node on $\mathcal{H}_{2}$ makes full rotation and then retreat to $\mathcal{H}_{3}$ rather than stop at its rotation starting point; in the next step the node returns to $\mathcal{H}_{2}$ again and starts the next rotation. As a result, a greedy-rotation collision loop occurs, and all nodes are rotating along $\mathcal{H}_{2}$ infinitively often. This collision loop is actually due to the problematic rotatingretreating role switch, which refreshes the rotating node's rotation record. It will not take place if we prevent the rotating node from being retreated outwards, which in turn can be achieved by enforcing the following ranking policy: a node that rotates is always assigned the highest rank in a local ranking process.

Notice that, whether the ranking policy is applied or not, collision loop never occurs in GRG-CV.

\subsection{Analysis}

In this section, we first prove the correctness of the two proposed algorithms GA and GRG. We prove that both GA and GRG are terminatable and that they yield a connected network with hole-free coverage. Afterwards, we analyze their coverage radius property. We derive that GA has no guarantee on coverage radius, and that GRG guarantees optimal or near optimal coverage radius.

\subsubsection{Correctness}

Lemma 3.1. Both $G A$ an $G R G$ ensure that $\Pi$ be occupied by a single node within finite time.

Proof. By the alignment rule, $\Pi$ could be occupied by multiple nodes during the initial node alignment. If $\Pi$ is still empty after the alignment process terminates, it will be eventually occupied by at least one node through greedy advance, because the algorithms ensure a winner 
in every competition for greedy advance. In any case, $\Pi$ becomes occupied within finite time. Once $\Pi$ is occupied, no node will move to it. If multiple nodes exist at $\Pi$ at some moment, one and only one of them will stay, and the others will move onto $\mathcal{H}_{1}$ according to the retreat rule. Hence, the lemma holds.

\section{Theorem 3.1. GA terminates within finite time.}

Proof. Because, after the initial node dropping, the distance from each node to its closest TT vertex is fixed, the node alignment process will terminate within finite time. By Lemma 3.1, $\Pi$ will be occupied by a single node within finite time. Henceforth, we safely assume that the deployment step already passed the alignment process and that $\Pi$ has been occupied by a single node.

When a node is leaving a TT vertex due to the retreat rule, the TT vertex will still be occupied by another node. The priority rule and the forbiddance rule prevent two nodes located at different TT vertices from greedily moving toward the same TT vertex. In summary, the number of occupied TT vertices never decreases.

Assume for the sake of contradiction that GA never terminates. Since the number of occupied TT vertices never decreases, it follows that there exists an $m \leq n$ where $n$ is the network size such that the algorithm runs infinitively long on $m$ occupied TT vertices.

Consider that after a finite number of deployment steps the TT vertices $T=\left\{t_{1}, \ldots, t_{m}\right\}$ are occupied. In subsequent steps, the set $T$ may only change due to a greedy rule. Assume for the sake of contradiction that $T$ changes due to the retreat rule. Whenever an unoccupied TT vertex is visited by the retreat rule, the number of occupied TT vertices increases by one, contradicting the assumption that GA runs infinitively long on $m$ occupied TT vertices.

Define by $\Sigma(T)$ the sum of the TT distance from $\Pi$ to the TT vertices in $T$, i.e., $\Sigma(T)=$ $\sum_{t_{i} \in T}\left|\operatorname{Path}_{S}\left(t_{i}, \Pi\right)\right|$. Whenever $T$ changes to $T^{\prime}$ due to a greedy rule, a node moves from a hexagon $\mathcal{H}_{i+1}$ to a hexagon $\mathcal{H}_{i}$. It follows, $\Sigma\left(T^{\prime}\right)=\sum(T)-1$. Since $\Sigma(T) \geq 0$, it follows that the set of occupied TT vertices can only change a finite number of times.

Let $F=\left\{f_{1}, \ldots, f_{m}\right\}$ be the final set of TT vertices visited by GA, i.e., $F$ no longer changes 
in subsequent steps. Subsequent deployment steps are only due to the retreat rule since a greedy rule will always visit a non-occupied TT vertex and would thus change the final set $F$.

Define $d$ as the maximum distance between $\Pi$ and the finally occupied TT vertices, i.e., $d=\max \left\{\left|\operatorname{Path}_{S}\left(f_{i}, \Pi\right)\right|\right\}, f_{i} \in F$. Let $Q=\left\{q_{1}, \ldots, q_{n}\right\}$ be the multi set of the TT vertices occupied by the sensor nodes, i.e. $q_{i}$ is the TT vertex occupied by sensor node $n_{i}$. Nodes moving due to the retreat rule always move from a hexagon $\mathcal{H}_{i}$ to a hexagon $\mathcal{H}_{i+1}$. Thus, a change from $Q$ to $Q^{\prime}$ always satisfies $\sum\left(Q^{\prime}\right)=\Sigma(Q)+1$.

It follows that after a finite number of deployment steps the multi set $Q$ of occupied TT vertices satisfies $\sum(Q)>n d$, i.e., there exists an occupied TT vertex $t$ which satisfies $\left|\operatorname{Path}_{S}(t, \Pi)\right|>d$. Thus, the visited TT vertex $t$ is not an element of $F$ which finally contradicts the assumption that $F$ is the final set of visited TT vertices.

Lemma 3.2. Let $\mathcal{H}_{0}, \cdots, \mathcal{H}_{i-1}$ be fully occupied without co-located nodes. Let $n \geq v(i)$. Then, in $G R G, \mathcal{H}_{i}$ will be fully occupied without co-located nodes within finite time.

Proof. When $\mathcal{H}_{0}, \cdots, \mathcal{H}_{i-1}$ are all fully occupied, nodes that have decided to stay on $\mathcal{H}_{i}$ never leave $\mathcal{H}_{i}$ but counterclockwise rotate along $\mathcal{H}_{i}$ because they are assigned highest rank in any local ranking process triggered by node collision, making unoccupied $\mathcal{H}_{i}$ vertices "rotating" in the opposite direction. In worst case, they make a full rotation and then stop moving forever, rendering unoccupied vertices fixed. In any case, after reaching $\mathcal{H}_{i+1}$, nodes are guaranteed to meet the empty vertices of $\mathcal{H}_{i}$ (by counterclockwise rotation) and move to fill their location by the suspension rule and the competition rule. Because $n \geq v(i)$ and there are no co-located nodes on the $i-1$ inner hexagons, $\mathcal{H}_{i}$ will be fully occupied at the end as nodes keep moving toward it and eventually stop on it. Nodal retreat guarantees that no $\mathcal{H}_{i}$ vertex be occupied by multiple nodes. Hence, the lemma holds.

Lemma 3.3. Let $\mathcal{H}_{0}, \mathcal{H}_{1}, \cdots, \mathcal{H}_{i-1}$ be fully occupied without co-located nodes. Let $v(i-1)<$ $n<v(i)$. Then, in $G R G$, nodes located on $\mathcal{H}_{i}$ will stop moving within finite time.

Proof. As the inner hexagons $\mathcal{H}_{0}, \mathcal{H}_{1}, \cdots, \mathcal{H}_{i-1}$ are all fully occupied, the nodes from outer 
hexagons will rotate along $\mathcal{H}_{i}$, after arriving $\mathcal{H}_{i}$. This is the case for both GRG-CV and GRG$\mathrm{CW}$. Note that, in GRG-CW, these rotating nodes could collide with some greedily advancing nodes, but their rotation is not affected (refer to Section 3.4.4). Because $v(i-1)<n<v(i)$, at lease one of them will make a full rotation. By protocol definition, this node will then stop moving forever, which will in turn block the rotation of any following node. Eventually, the nodes on $\mathcal{H}_{i}$ will become fixed. After the nodes on $\mathcal{H}_{i}$ stops moving, the nodes on $\mathcal{H}_{i+1}$ (if any) will get onto $\mathcal{H}_{i}$ and possibly rotate along $\mathcal{H}_{i}$ as well. These newly arriving nodes will stop moving and become fixed within finite time because of the blocking from previously stopped nodes on $\mathcal{H}_{i}$. Hence the lemma holds.

Theorem 3.2. GRG terminates within finite time.

Proof. It follows immediately from Lemmas 3.1-3.3.

Theorem 3.3. Both $G A$ and $G R G$ yields a connected network with hole-free coverage.

Proof. We prove this theorem by contradiction. By Theorem 3.1 and 3.2, we known that both GA and GRG terminates within finite time. Assume that there is a sensing hole in the coverage region at some moment after the algorithm (either GA or GRG) terminates. Denote by $v$ a vertex farthest from $\Pi$ on the border of the hole and by $\langle i, j, k\rangle$ the address of $v$. There must exist a node at $\langle i+1, j, k\rangle$ (or $\langle i+1, j, i\rangle$ if $k=0$ ), because, otherwise, $v$ would not be the farthest border vertex of the hole. In this case, that node will greedily proceed to occupy $v$ by protocol definition. This actually contradicts our assumption that the algorithm has terminated. Thus the final coverage constructed by the algorithm (either GA or GRG) contains no sensing hole. Then network connectivity simply follows from the lack of sensing holes and the assumption of $r_{c} \geq \sqrt{3} r_{s}$ (see Section 3.2). Hence the theorem holds.

\subsubsection{Coverage radius}

Recall that coverage radius can be measured using different measurements, i.e., layer distance or Euclidean distance. In GA, the final coverage of a mobile sensor network has an 
unpredictable shape, depending very much on initial sensor placement. As shown in Figure 3.6(a), it is possible that $\Pi$ is located on the border of the network, rendering coverage radius equal to 0 . This example implies that GA provides no coverage radius guarantee in neither layer distance nor Euclidean distance. In contrast, as we will see, GRG generates optimal or near optimal focused coverage in both metrics.

Consider a mobile sensor network of size $n$. Let $\gamma_{o p t}^{H}$ be the optimal hexagonal coverage radius (measured in layer distance) that the network can provide. Then we have $\gamma_{o p t}^{H}=\left\lfloor v^{-1}(n)\right\rfloor$ where $v^{-1}()$ is the inverse function of $v()$ (Equation 3.1 in Section 3.3), that is,

$$
v^{-1}(n)=\frac{\sqrt{12 n-3}-3}{6}
$$

Let $\mathcal{F}$ be the focused coverage constructed by GRG using this network. Denote by $\gamma^{H}$ the hexagonal radius of $\mathcal{F}$. From Lemmas $3.1-3.3$, the following optimality result follows regardless of the network size $n$.

Theorem 3.4. In GRG, $\gamma^{H}=\gamma_{o p t}^{H}$.

We will now study the circular radius $\gamma^{C}$ (measured in Euclidean distance) of $\mathcal{F}$. Denote by $\gamma_{o p t}^{C}$ the optimal circular coverage radius that the network can provide. Further, let $S$ be the size (area) of $\mathcal{F}$, and $\mathcal{H}_{\kappa}$ the outmost hexagon of $\mathcal{F}$, where $\kappa=\left\lceil\nu^{-1}(n)\right\rceil$. We first derive bounds on $\gamma_{o p t}^{C}$.

Theorem 3.5. $\frac{3}{2}(\kappa-1) r_{s} \leq \gamma_{o p t}^{C} \leq 3 \sqrt{\frac{\sqrt{3}}{2 \pi}} \kappa r_{s}$.

Proof. The lower bound, which is the radius of the inscribed circle of $\mathcal{H}_{\kappa-1}$ is obvious. Recall the definition of coverage. It is provable that the hexagonal node placement produces maximized coverage over the TT. In this case, $\gamma_{\text {opt }}^{C}$ must not be larger than the radius of the circle whose area is equal to the area of $\mathcal{H}_{\kappa}$, that is, $\gamma_{o p t}^{C} \leq 3 \sqrt{\frac{\sqrt{3}}{2 \pi}} k r_{s}$.

Then we shall show GRG yields optimal or near optimal circular coverage radius, depending on the network size $n$. We have to examine two cases: 


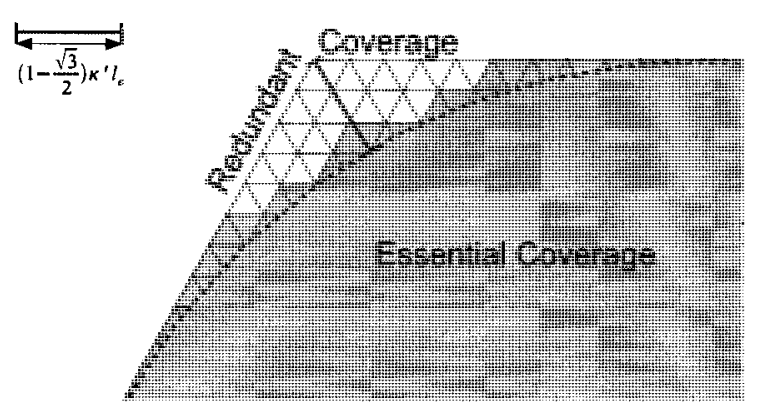

Figure 3.8: Redundancy

(1) $n=v(\kappa)$, meaning that, $\mathcal{H}_{\kappa}$ is fully occupied;

(2) $n \neq \nu(\kappa)$ (precisely $v(\kappa-1)<n<v(\kappa)$ ), meaning that, $\mathcal{H}_{\kappa}$ is partially occupied.

Lemma 3.4. In $G R G, 0.95 \gamma_{o p t}^{C} \leq \gamma^{C} \leq \gamma_{o p t}^{C}$ for $n=v(\kappa)$.

Proof. In the case of $n=v(\kappa), \gamma^{C}$ is equal to the radius of the inscribed circle of $\mathcal{H}_{\kappa}$, namely, $\gamma^{C}=\frac{3}{2} \kappa r_{s}$. By Theorem 3.5, $\frac{\gamma^{C}}{\gamma_{o p t}^{C}} \geq \frac{\frac{3}{2} \kappa r_{s}}{3 \sqrt{\frac{\sqrt{3}}{2 \pi}} \kappa r_{s}}=\sqrt{\frac{\pi}{2 \sqrt{3}}}=0.95$. And obviously, $\gamma^{C} \leq \gamma_{o p t}^{C}$. Hence, the lemma holds.

Lemma 3.5. In $G R G, 0.95 \frac{\kappa-1}{\kappa} \gamma_{o p t}^{C} \leq \gamma^{C} \leq \gamma_{o p t}^{C}$ for $n \neq v(\kappa)$.

Proof. When $n \neq v(\kappa), \gamma^{C}$ must not be less than the radius of the inscribed circle of $\mathcal{H}_{\kappa-1}$, i.e., $\gamma^{C} \geq \frac{3}{2}(\kappa-1) r_{s}$. By Theorem 3.5, $\frac{\gamma^{C}}{\gamma_{o p t}^{C}} \geq \frac{\frac{3}{2}(\kappa-1) r_{s}}{3 \sqrt{\frac{\sqrt{3}}{2 \pi}} \kappa r_{s}}=\frac{\kappa-1}{\kappa} \sqrt{\frac{\pi}{2 \sqrt{3}}}=0.95 \frac{\kappa-1}{\kappa}$. Obviously, $\gamma^{C} \leq \gamma_{o p t}^{C}$. Hence, the lemma holds.

Summarizing Lemmas 3.4 and 3.5, we have the following theorem:

Theorem 3.6. Let $\delta=\left\{\begin{array}{ll}1, & n=v(\kappa) ; \\ 1-\frac{1}{\kappa}, & n \neq v(\kappa) .\end{array}\right.$ Then in $G R G, 0.95 \delta \gamma_{o p t}^{C} \leq \gamma^{C} \leq \gamma_{o p t}^{C}$

According to Theorem 3.6, it would appear that GRG was not able to produce good focused coverage when $n \neq v(\kappa)$ for a small $\kappa$. For instance, if $\kappa=2$, the lower bound will be $0.475 \gamma_{o p t}^{C}$, proportionally too far from the optimal value. It is however not true in reality, as indicated by the following complementary theorem:

Theorem 3.7. In $G R G, \gamma^{C}=\gamma_{\text {opt }}^{C}$ for $n=\nu(\kappa) \wedge \kappa<29$ and for $n \neq \nu(\kappa) \wedge \kappa<15$. 
Proof. Let $\mathcal{H}_{\kappa^{\prime}}$ be the outmost fully-occupied hexagon of $\mathcal{F}\left(\kappa^{\prime}=\kappa\right.$ when $n=\nu(\kappa)$, or $\kappa^{\prime}=\kappa-1$ when $n \neq v(\kappa)$ ). Denote the inscribed disc of $\mathcal{H}_{\kappa^{\prime}}$ by $\mathcal{C}_{\kappa^{\prime}}$. Apparently, $\gamma^{C}$ must not be smaller than the radius $R\left(C_{\kappa^{\prime}}\right)$ of $C_{\kappa^{\prime}}$. From guaranteed coverage viewpoint, we consider $\gamma^{C} \equiv R\left(C_{\kappa^{\prime}}\right)$. As the final network has the TT layout, it is the TT triangles (in fact, their comprising nodes) inside $C_{\kappa^{\prime}}$ and those crossing the border that contribute to the accomplishment of $\gamma^{C}$. Hence, the coverage corresponding to these triangles constitute the essential coverage disc ( $E C D)$, while the rest is redundant.

Figure 3.8 shows a corner area of $\mathcal{H}_{\kappa^{\prime}}$ with essential coverage marked in gray color. Through trivial calculation, we can derive that the hexagons that contribute to the redundant coverage are $\mathcal{H}_{\kappa^{\prime}}, \mathcal{H}_{\kappa^{\prime}-1}, \cdots, \mathcal{H}_{\kappa^{\prime}-\tau\left(\kappa^{\prime}\right)+1}$, where $\tau\left(\kappa^{\prime}\right)$ solely depends on $\kappa^{\prime}$ as follows:

$$
\tau\left(\kappa^{\prime}\right)=\left\lceil\frac{\left(1-\frac{\sqrt{3}}{2}\right) \kappa^{\prime} l_{e}}{l_{e}}-1\right\rceil=\left\lceil\left(1-\frac{\sqrt{3}}{2}\right) \kappa^{\prime}-1\right\rceil
$$

Let $t$ be an integer that falls in range $\left[1, \tau\left(\kappa^{\prime}\right)\right]$. Further, we can find the exact number of nodes that are enclosed inclusively by $\mathcal{H}_{\kappa^{\prime}}$ and contribute to the redundant coverage is

$$
\rho\left(\kappa^{\prime}\right)=6 \sum_{t}\left(2\left\lfloor\frac{1}{2}\left(\kappa^{\prime}-t-\sqrt{6 \kappa^{\prime} t-3 t^{2}}\right)\right\rfloor+1\right)
$$

Notice the implicit restriction $\tau\left(\kappa^{\prime}\right) \geq 1$ on the above equation. Solving this inequality, we get $\kappa^{\prime} \geq 7.4$, which actually implies that there is no node redundancy for $\kappa^{\prime} \leq 7$. Hence the total number $\mu\left(\kappa^{\prime}\right)$ of nodes that comprise the ECD will be

$$
\mu\left(\kappa^{\prime}\right)= \begin{cases}v\left(\kappa^{\prime}\right) & \text { for } \kappa^{\prime} \leq 7 \\ v\left(\kappa^{\prime}\right)-\rho\left(\kappa^{\prime}\right) & \text { for } \kappa^{\prime}>7\end{cases}
$$

When the number of redundant sensors, i.e., $n-\mu\left(\kappa^{\prime}\right)$, is large enough, $\gamma^{C}$ can be increased by arranging the redundant sensors around the essential coverage along the TT grid. Algorithmic detail about how to achieve this improvement will be presented in Chapter 4 . 
More specifically, coverage radius improvement is possible only when $n \geq \mu(\kappa+1)$ in the case of $n=v(\kappa)$, or $n>\mu(\kappa)$ in the case of $n \neq v(\kappa)$. By an exhaustive search, it is provable that the minimum $\kappa$ in the above two cases are respectively 29 and 15.

From Theorems 3.6 and 3.7, the following corollary follows:

Corollary 3.1. In $G R G, 0.88 \gamma_{o p t}^{C} \leq \gamma^{C} \leq \gamma_{o p t}^{C}$ in the worst case for $n \neq v(\kappa) \wedge \kappa \geq 15$.

\subsection{Performance Evaluation}

Although sensor self-deployment is not a new topic in general, sensor self-deployment for focused coverage formation around a POI is to our best knowledge a new problem addressed for the first time in this thesis. Existing sensor self-deployment algorithms follow a different objective, i.e., coverage formation over a ROI, and may yield a network with coverage radius as bad as 0 . As we emphasize on optimizing coverage radius, these algorithms are clearly not comparable to ours. Thus below we will evaluate GA and GRG alone.

\subsubsection{Evaluation metrics}

We study the performance of GA and GRG in the following three aspects: convergence time, energy consumption and node collision. Because GA and GRG assume that nodes obtain their neighborhood information from lower layer protocols, and because they themselves do not generate any message during the course of sensor self-deployment, communication cost is not our concern here.

\section{Convergence Time}

Convergence Time $(C T)$, also known as deployment latency, is defined as the number of time units that it takes a self-deployment algorithm to yield a stabilized network (with no floating nodes). Convergence time is heavily subject to nodes' initial distance to the POI. If 
nodes are placed arbitrarily far, CT can be an unbounded value. Node velocity is another impact factor. Slow-moving nodes will slow down deployment speed, rendering CT very large.

Normally, a sensor self-deployment algorithm converges only when all the nodes become still. Let $\mathcal{H}_{\kappa}$ be the outmost hexagon of a focused coverage generated by GRG. If $\mathcal{H}_{\kappa}$ is partially occupied (i.e., $n \neq v(\kappa)$ ), the nodes residing on it may or may not contribute to coverage radius maximization, depending very much on their final distribution and the value of $\kappa$. In this case, from guaranteed coverage point of view, it is reasonable and acceptable to consider that GRG converges as long as the $\kappa-1$ inner hexagons are fully filled.

\section{Energy consumption}

Energy consumption is measured using Number of Moves (NM) and Mileage (MG). The former is defined as the number of times that a node restarts its motor. It matters because starting a still motor consumes a fairly large amount of energy. The latter is defined as the total distance that a node has traveled during the course of its self-deployment. It is subject to a node' initial distance to the POI to large extent.

In addition to total energy consumption, we particularly evaluate the part of energy used for coverage shape control, one of the unique features of GRG. To do so, we introduce another metric - Mileage over Progress ratio (MP). A node's progress (PG) equals to the absolute value of the subtraction of its initial Euclidean distance $D_{i n i}$ to the POI from its final Euclidean distance $D_{\text {fin }}$ to the POI.

\section{Node collision}

Collision does not necessarily mean that two nodes physically collide at a geographic point. In fact, we may consider that two nodes collide as long as they are located sufficiently close to each other. Collision is due to randomized initial node placement as well as algorithmic design. Although collision appears as transient phenomenon in both GA and GRG, 
it matters because it could bring colliding nodes radio signal interference at physical layer, causing various communication failures.

We say that a node conducted a wasted greedy step if it performs a retreat step immediately after. Apparently, wasted greedy movement increases both convergence time and energy consumption and therefore degrade algorithm performance. Since wasted greedy movement results only from node collision, measuring the Number of Collisions (NC) during sensor self-deployment can help further clue in algorithm performance.

\subsubsection{Simulation setup}

We implemented GA and GRG (including the two variants GRG-CW and GRG-CV) within a custom network simulator, and simulated their execution over a mobile sensor network stochastically dropped in two-dimensional free plane. In our simulation, the geographic center of dropping area is taken as POI. Nodes are equipped with sensing radius 10 and communication radius $10 \times \sqrt{3} \approx 18$; they may move at different speeds, ranging from 0.05 to 0.2 per simulated time unit, for their every single step.

We conducted two sets of experiments. In the first set, we wish to study the performance of the two algorithms under different node density by fixing the size SZ of dropping area to $200^{2}$ and varying network size $n$ from $v(1)=7$ to $v(10)=331$. In the second set, we aim to evaluate the two algorithms with different average initial node distance by fixing $n$ to $v(7)=169$ and varying SZ from $0^{2}$ (which means that nodes are all placed at the POI at initiation) to $500^{2}$. In order to minimize data noise, for each simulation setting, we executed GA and GRG over 50 randomly generated network scenarios and computed average results for analysis.

\subsubsection{Experimental results}

In the sequel, we will elaborate our experimental results displayed in Figure 3.9 and 3.10. We first focus on the set of experiments that take varied-sized network with fixed-sized dropping area, and the we turn our attention to the other set of experiments that use fixed-sized 


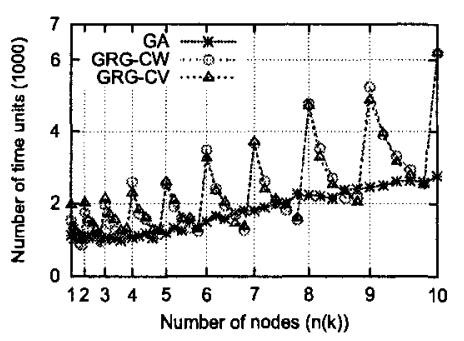

(a) Convergence time

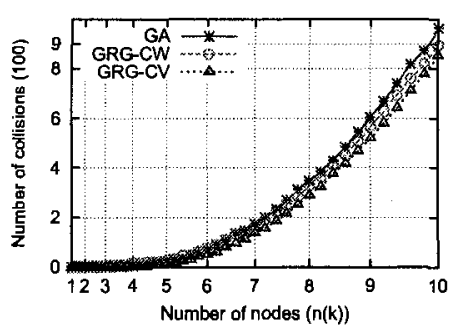

(d) Number of collisions

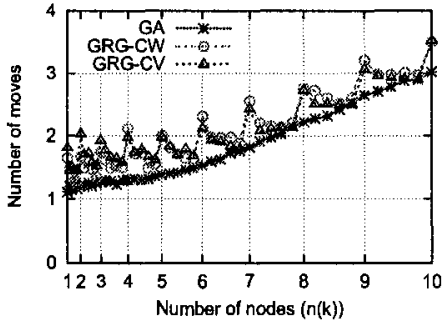

(b) Number of moves per node

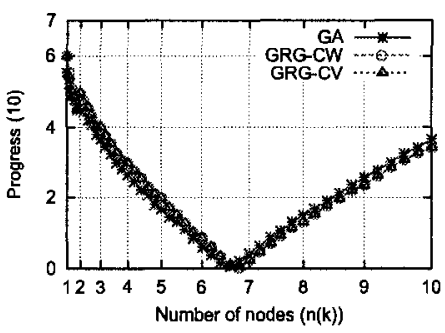

(e) Progress per node

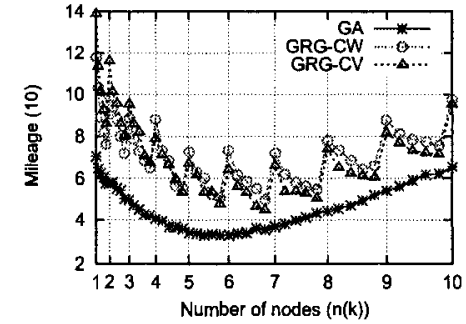

(c) Moving distance (mileage) per node

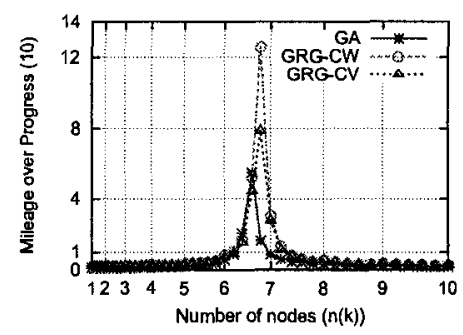

(f) Ratio of mileage over progress

Figure 3.9: Results from a network of size varying from $v(1)$ to $v(10)$ and a dropping area of size fixed to $200^{2}$

network with varied-sized dropping area. As we shall see below, GA outperforms GRG in the aspects of convergence time and energy consumption, and GRG-CV is more suitable for dense networks compared with GRG-CW.

\section{Varied-sized network with fixed-sized field}

Examine Figure 3.9(a) and 3.9(b), which respectively indicate CT and NM as a function of $n$. They contain curves of similar trend. We first investigate the monotonically increasing curves of GA in the two figures. When $n=v(1)$, the network is very sparse and has a very small size 7 . In such a network, greedy advance overwhelmingly dominates the selfdeployment process, and nodes are able to continuously move most of time without frequently (or with even no) being blocked and waiting, resulting in low-valued CT and NM. As $n$ increases, the frequency of both blocking and retreat movement rises, and waiting and resuming happen more and more often. In this case, both CT and NM increase as a result.

Now, let us look at the curves corresponding to GRG-CW and GRG-CV in the two figures. 
If we link the points with $n=v(\kappa)$, we get two closely-located monotonically-increasing curves in both figures. In either figure, the two new curves are both located above the curve for GA. It is because GRG involves an extra type of node movement, rotation, which complexes the self-deployment process. Observe any interval between $v(\kappa-1)$ and $v(\kappa)$ for an integer $\kappa$, and we find that the curve of either variant of GRG descends in this interval, which is actually reasonable. In the case of $n=v(\kappa)$, GRG does not converge until the outmost hexagon is fully occupied; in any other case, it, as we mentioned in Section 3.6.1, converges as soon as all the inner $\kappa-1$ hexagons are fully filled, making a dramatic decrease of both CT and NM. In fact, when $n$ is very close to $v(\kappa)$, GRG performs even better than GA, as shown in the two figures, since the latter converges only when nodes all stop moving.

Figure 3.9(c) illustrates how MG varies as $n$ changes. It is observed that the curves for GRG-CW and GRG-CV have a declining trend when $n$ lies in the range between $v(\kappa-1)$ and $v(\kappa)$ for an integer $\kappa$. This phenomenon is due to exactly same reason as the similar phenomena observed in Figure 3.9(a) and 3.9(b). If we link the points on GRG curves with $n=v(\kappa)$ together, we also get two closely-located monotonically-increasing curves. The two new curves surpass the curve for GA for every value of $n$ because GA does not generate rotation movement. Besides, they also have the same trend as GA: firstly declining and then climbing. It is because, as $n$ goes up, the network becomes increasingly dense, and $D_{\text {fin }}$ thereby increases and approaches $D_{i n i}$, which in turn makes nodes travel a decreased distance. But, after node density is beyond a saturated value (which is reached when $n$ is around $v(6)$ ), the network shows an expanding behavior, namely, that nodes move outwards for coverage maximization, leading to the monotonic increase of MG with increased $n$.

Closely examine the three figures 3.9(a) - 3.9(c) again. We can find that GRG-CW performs better in sparse networks, but worse in dense networks, than GRG-CV. This phenomenon is arguable. When $n$ is small, greedy advance dominates sensor self-deployment, and node collision, which has obvious negative impact on CT, NM and MG, happens rarely. In this case, aggressive GRG-CW beats conservative GRG-CV, as the latter often unnecessar- 
ily forces nodes to travel increased distance. As $n$ mounts up, the network shows more and more a rotating or expanding behavior, and node collision occurs increasingly often, as confirmed by Fig. 3.9(d) and discussed in next paragraph. The positive impact of the strict hop selection rules of GRG-CV keeps growing, while their negative effect constantly decrease, finally rendering GRG-CV outperform GRG-CW.

Figure 3.9(d) shows NC in relation with $n$. Observe that NC keeps ascending as $n$ increases because the probability of node collision climbs as node density, which is proportional to network size in the case of fixed-sized dropping area, increases. Also observe that GRG-CV always yields smaller NC than GRG-CW. Note that GRG-CV itself does not cause any node collision and that collision occurs during its execution only for the sake of randomized initial node placement. As shown in the figure, GA and GRG have nearly the same performance in a small-sized network, and that they deviate from each other as $n$ goes up. The performance of GRG-CV is below that of GA in all cases because rotation helps reduce retreat-related collision. The performance of GRG-CW is first above that of GA because GRG-CW generates a large proportion of greedy-rotation collisions in a sparse network with concentrating behavior, and then gets below it (after $n=v(6)$ ) because the proportion of greedy-rotation collision diminishes, and that of retreat-related collision avoided by rotation contrarily emerges.

Figure 3.9(e) illustrates PG as a result of $n$. The curves corresponding to GA and the two versions of GRG are all in a "V" shape with the lowest point rooted between $n=v(6)$ and $n=v(7)$. They imply that this particular value of $n$ makes the network reach a saturated status, namely $D_{i n i}$ is roughly equal to $D_{f i n}$ such that nodes make no (large) progress during the course of self-deployment. In such a network, the network shows a rotating behavior in general. When $n$ deviates more and more from $v(7)$, the difference between $D_{i n i}$ and $D_{f i n}$ becomes bigger and bigger, resulting in the rise of NC. Note that the network shows a concentrating behavior when $n<v(7)$ and an expending behavior when $n>v(7)$.

Figure 3.9(f) exhibits MP versus $n$. It is observed that MP is lower than 10 and actually very close to 1 for both GA and GRG almost for all the values of $n$. In the figure, MP reaches 


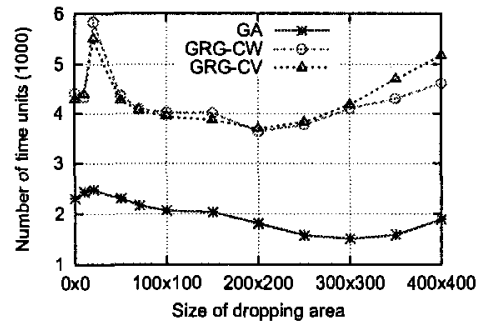

(a) Convergence time

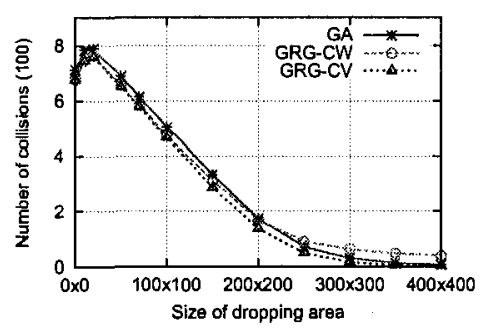

(d) Number of collisions

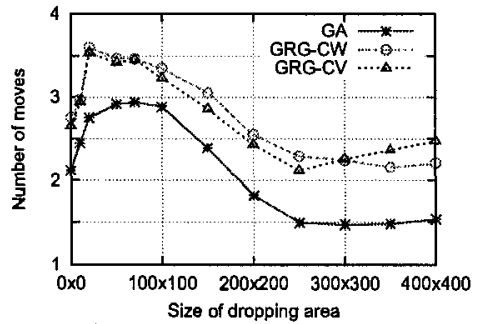

(b) Number of moves per node

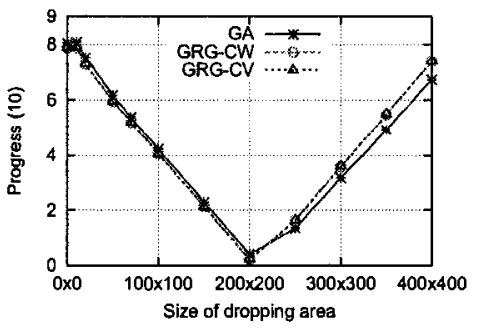

(e) Progress per node

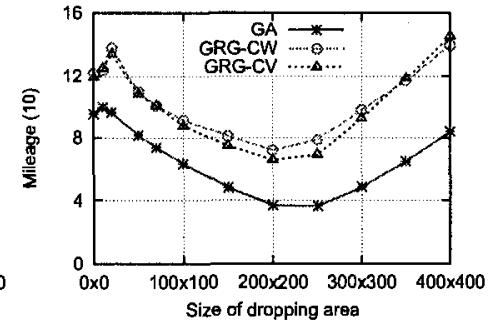

(c) Moving distance (mileage) per node

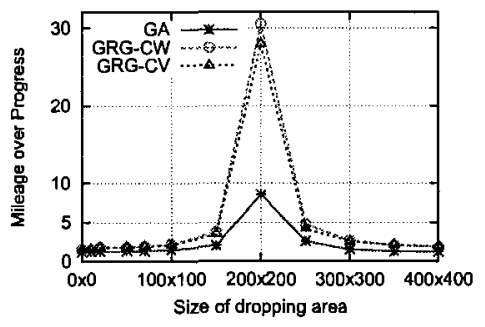

(f) Ratio of mileage over progress

Figure 3.10: Results from a network of size fixed to $v(7)$ and a dropping area of size varying from $0^{2}$ to $400^{2}$

its peak value at the point around $n=v(7)$. As a matter of fact, MP can go to infinity in the case of $P=0$. Although this extreme situation may not come into reality, but it is possible in theory, for example, when all the nodes are by any change located at the right deployment points at initiation. Additionally, it is observed that MP decreases and approaches 1 closer and closer as $n$ increases or decreases toward the two end values. Through a comparative study on the two figures 3.9(c) and Figure 3.9(e), the reason for this phenomenon becomes fairly obvious: PG has a way smaller value (nearly equal to 0 ) than MG round $n=v(7)$ and it climbs at a much faster speed than MG with increased/decreased $n$.

\section{Fixed-sized network with varied-sized field}

Figures $3.10(\mathrm{a})-3.10$ (c) respectively show $\mathrm{CT}$, NM and $\mathrm{MG}$ as a function of $\mathrm{SZ}$. The curves in the three figures share a similar trend. When nodes are all located at the POI (i.e., when $\mathrm{SZ}=0^{2}$ ), they spread out with equal probability in every direction along TT edges. In this simple scenario, neither rotation nor greedy advance often occurs, and nodes are very 
likely to travel a short distance to their final position through continuous retreat movement (moving away from the POI). As SZ increases, rotation and greedy advance take place more and more frequently, complexing the self-deployment process. In such a complicated situation, nodes are prone to move intermittently and perform wasted greedy advance, rendering the increase of CT, NM and MG. As shown in the figures, the curves keep ascending until SZ reaches certain value about $20^{2}$ and then descends thereafter. It is because SZ gradually becomes large enough for continuous node movement and for reducing the possibility of wasted greedy advance. But, after the network becomes sufficiently sparse (i.e., $\mathrm{SZ}>200^{2}$ or $250^{2}$ ), the curves rise again because nodes block each other during the course of their concentrating self-deployment, leading to large amounts of non-progressive rotation, frequent stops and therefore increased waiting time.

In the three figures $3.10(\mathrm{a})-3.10(\mathrm{c})$, GA is always located below GRG due to its algorithmic simplicity. It is also observed that GRG-CV stays below GRG-CW before $\mathrm{SZ}=300^{2}$ and surpasses it thereafter as SZ increases. The reason for this phenomenon is rooted at the semi-greedy nature of GRG-CV. As we know, GRG-CV generates no greedy-rotation collision due to its strict greedy rules, and it therefore yields less wasted greedy advance compared with GRG-CW. Under this circumstance, GRG-CV outperforms GRG-CW in a dense network $\left(\mathrm{SZ}<300^{2}\right.$ ), where greedy advance does not occur as often as other types of movement; in an adequately sparse network $(\mathrm{SZ}>350)$ where greedy advance is prevailing, GRG-CW however performs better than GRG-CV because the latter yields more frequent stop and nonprogressive rotation.

Figure 3.10(d) illustrates NC versus SZ. When SZ $=0^{2}$, collision-prone retreat movement overwhelmingly dominates the self-deployment process and gives rise of high-valued NC. With a slight increase of SZ, the occurrence frequency of retreat movement does not change apparently, but rotation and greedy happen relatively much often, leading to the rise of NC. However, as shown in the figure, this rising trend exists only within a small range of SZ, from $0^{2}$ to $50^{2}$. Note that the boosting phenomenon in this $\mathrm{SZ}$ range happens to other metrics 
like CT, NM and MG as well (refer to Figure 3.10(a) - 3.10(c)). After SZ is beyond $50^{2}$, retreat movement gradually loses its dominating role, and retreat-related collision becomes less and less possible, inducing the monotonic drop of NC. In fact, if the network is very sparse, the nodes close to the POI could possibly stop moving before remote ones arrive, leading to $\mathrm{NC}=0$. In a dense network $\left(\mathrm{SZ}<200^{2}\right)$, rotation helps reduce the probability of retreatrelated collision, making GRG outperforms GA. In a sparse network (SZ > 200²), GRG-CW has worse performance than GA because it can generate extra greedy-rotation collisions, and GRG-CV stays superior to GA as it brings no additional collisions (it in fact prevents greedy collision at $\Pi$ ).

Recall that $\mathrm{PG}=\left|D_{\text {ini }}-D_{\text {fin }}\right|$. Because $n$ stays unchanged, the average node final distance $D_{\text {fin }}$ to the POI is fixed, and PG depends solely on $D_{i n i}$, which is in turn subject to the size of dropping area. Figure 3.10(e) shows PG in relation with SZ. It is observed that, for both GA and GRG, PG is lowest (nearly equal to 0 ) when $\mathrm{SZ}=200^{2}$, and that it rises as $\mathrm{SZ}$ approaches the two end values, rendering the curves in " $V$ " shape. This phenomenon indicates that the closer SZ is to $200^{2}$, the closer $D_{i n i}$ is to $D_{\text {fin }}$. We can see that the two variants of GRG have the same performance, and that they always yield larger PG than GA. We can also find that the gap between GRG and GA becomes larger and larger after SZ exceeds $200^{2}$. It is because rotation increases the chance of nodes for greedy advance, especially in a sparse network. Note that the curves in this figure do not match those in Figure 3.10(e) but show a sharper change, because nodes do not move straight to their final position but through curly paths in the TT, especially in GRG that involves rotation.

Figure 3.10(f) exhibits MP in relation with SZ. It is observed that MP is lower than 5 (in fact, very close to 1) for both GA and GRG almost for all the values of SZ. The figure shows that MP reaches its peak value when $S Z=200^{2}$. However, there is no maximum value for MP since PG could be equal to 0 in theory. Additionally, it is displayed that MP decreases and approaches 1 closer and closer as SZ increases or decreases toward the two end values. Through a comparative study on Figure 3.10(c) and 3.10(e), the reason for this phenomenon 
becomes fairly obvious: PG has a way smaller value (nearly equal to 0 ) than $\mathrm{MG}$ at $\mathrm{SZ}=200^{2}$, and it climbs at a much faster speed than MG with SZ increased/decreased from $200^{2}$.

\subsection{Summary}

Sensor self-deployment is an emerging research problem. It requires localized solutions that provide coverage guarantee. To our knowledge, no such a sensor self-deployment algorithm exists in the literature. In this chapter, we pinpointed a new sensor self-deployment problem, focused coverage formation around a Point of Interest (POI) and introduced an evaluation metric, coverage radius. We proposed two solution algorithms, Greedy Advance (GA) and Greedy-Rotation-Greedy (GRG), which are resilient to node failures and able to work regardless of network partition.

Both GA and GRG generate a network with an equilateral triangle tessellation layout and with no sensing hole. They are the first localized sensor self-deployment algorithms that provide such coverage guarantee. We proved their correctness and analyzed their coverage radius property. Our study shows that GA does not guarantee maximized coverage radius, and that GRG however ensures optimal hexagonal coverage radius and (near) optimal circular coverage radius. We also evaluated, through an extensive set of simulation, the performance of GA and GRG on convergence time, energy consumption and node collision. 


\section{CHAPTER 4 FOCUSED COVERAGE BY MOBILE SENSORS II}

4 sensors are required to autonomously surround a coverage focus, called Point of Interest (POI). In Chapter 3, we presented a strictly localized solution algorithm GRG. This algorithm is proven to generate an optimal hexagonal focused coverage, which may however not be optimal in terms of circular coverage radius. An optimal circular coverage radius has maximized circular radius. In this chapter, we modify GRG so as to achieve this optimality guarantee.

The modified version of GRG is referred to as GRG ${ }^{\star}$. It follows the same combined greedy-rotation philosophy as GRG for sensor self-deployment; yet it differs from GRG in its employed polygonal sensor rotation trajectories that best approximate circles. Through an extensive set of simulation, we comparatively evaluate $G_{R G}^{\star}$ with GRG in the aspects of convergence time, energy consumption and node collision.

In Section 4.1, we introduce focused coverage and algorithm GRG at very short length, and describe the working principle of $\mathrm{GRG}^{\star}$ briefly; in Section 4.3, we define deployment polygons to be used by $\mathrm{GRG}^{\star}$ for sensor rotation; in Section 4.4, we present the algorithmic detail of $\mathrm{GRG}^{\star}$; in Section 4.5 , we evaluates $\mathrm{GRG}^{\star}$ in comparison with GRG through extensive simulation; Section 4.6 summarizes the chapter. 


\subsection{Introduction}

In Chapter 3, we presented a strictly localized sensor self-deployment algorithm GreedyRotation-Greedy (GRG) for autonomous focused coverage formation. GRG generates an optimal focused coverage of maximized hexagonal radius. But it does not guarantee optimal circular coverage, even though the resulting circular radius closely approaches the maximum value (with a factor in $[0.88,1]$ ). In this chapter, we will address how to modify GRG so as to achieve guaranteed circular coverage radius maximization.

\subsubsection{Our contributions}

We propose an optimized Greedy-Rotation-Greedy algorithm $\mathrm{GRG}^{\star}$ for guaranteed circular coverage radius maximization. Following the same philosophy as GRG, it drives sensors to move from vertex to vertex over a locally computed TT graph to surround the POI, greedily advancing and rotating in an alternate fashion. But nevertheless, unlike GRG where sensors rotate along concentric hexagons, it guides sensors to rotate along our so-called deployment polygons that best approximate circles, therefore always leading to an optimal focused coverage of maximized circular radius. Our main contributions in this chapter include:

- We discover deployment polygons over the TT graph that can be used to achieve maximized circular coverage radius and present their localized computation.

- We propose an optimized Greedy-Rotation-Greedy algorithm $\mathrm{GRG}^{\star}$ using deployment polygons, which produces an optimal focused coverage of maximized circular radius.

- We evaluate $\mathrm{GRG}^{\star}$ on its convergence time, energy consumption and node collision in comparison with GRG through extensive simulation. 
Table 4.1: Notations

\begin{tabular}{ll}
\hline $\mathcal{P}_{i}$ & The deployment polygon associated with $C_{i}$, best approximating $C_{i}$ in $G_{T T}$ \\
$\operatorname{Patt}(v)$ & The neighborhood pattern of vertex $v$, with respect to its residence polygons \\
$N^{l}(v)$ & The left-hand neighbor set of $v$, with respect to its residence polygons \\
$N^{r}(v)$ & The right-hand neighbor set of $v$, with respect to its residence polygons \\
$N^{i}(v)$ & The inward neighbor set of $v$, with respect to its residence polygons \\
$N^{o}(v)$ & The outward neighbor set of $v$, with respect to its residence polygons \\
$\operatorname{Inn}\left(N^{l}(v)\right)$ & The left-hand vertex neighbor of $v$ on its inner residence polygon \\
$\operatorname{Out}\left(N^{l}(v)\right)$ & The left-hand vertex neighbor of $v$ on its outer residence polygon \\
$\operatorname{Ltm}\left(N^{i}(v)\right)$ & The leftmost vertex neighbor of $v$ in $N^{i}(v)$ \\
$\operatorname{Mid}\left(N^{i}(v)\right)$ & The middle vertex neighbor of $v$ in $N^{i}(v)$ \\
$\operatorname{Rtm}\left(N^{i}(v)\right)$ & The rightmost vertex neighbor of $v$ in $N^{i}(v)$ \\
$\operatorname{Ltm}\left(N^{o}(v)\right)$ & The leftmost vertex neighbor of $v$ in $N^{o}(v)$ \\
$\operatorname{Mid}\left(N^{o}(v)\right)$ & The middle vertex neighbor of $v$ in $N^{o}(v)$ \\
$\operatorname{Rtm}\left(N^{o}(v)\right)$ & The rightmost vertex neighbor of $v$ in $N^{o}(v)$ \\
\hline
\end{tabular}

\subsection{Model and Definitions}

We follow the same network model, the same goal, and the same notations defined in Section 3.2. Some additional notations can be found in Table 4.1.

\subsection{Deployment polygon}

For a focused coverage produced by GRG, its circular coverage radius $\gamma^{C}$ obviously must not be smaller than the radius $R\left(C_{K^{\prime}}\right)$ of the inscribed circle $C_{k^{\prime}}$ of the outmost fully-occupied hexagon $\mathcal{H}_{\kappa^{\prime}}$. From guaranteed coverage point of view, we consider $\gamma^{C}$ is equivalent to $R\left(C_{\kappa^{\prime}}\right)$, i.e., $\gamma^{C} \equiv R\left(C_{\kappa^{\prime}}\right)$. As discussed in Section 3.5.2, $\mathcal{H}_{\kappa^{\prime}}$ may not approximate $C_{\kappa^{\prime}}$ best, and there may exist, in the corner areas of $\mathcal{H}_{K^{\prime}}$, nodes that make no contribution to the accomplishment of $\gamma^{C}$. For this node redundancy, GRG does not guarantee maximized circular coverage radius.

In this section, we define our so-called deployment polygons that best approximate circles 


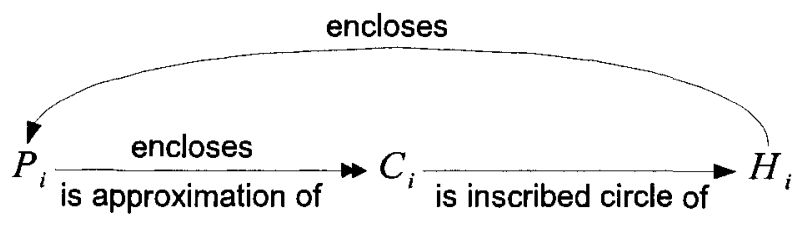

Figure 4.1: Relation diagram

in $G_{T T}$ and present their localized computation. Later, in Section 4.4, we will show how to modify GRG by using these polygons to ensure circular coverage radius maximization.

\subsubsection{Definition}

In discrete graph $G_{T T}, C_{i}$ is best approximated without radius reduction by a minimum polygon that encloses $C_{i}$ and consists of successive vertices. This polygon must be completely contained in or overlapped by $\mathcal{H}_{i}$, because, otherwise, $\mathcal{H}_{i}$ is a better approximation. We refer to this polygon as deployment polygon associated with $\mathcal{C}_{i}$ and denote it by $\mathcal{P}_{i}$. More formally, we define

Definition 4.1 (Deployment Polygon). In $G_{T T}$, a deployment polygon $\mathcal{P}_{i}$ is the perimeter of the polygonal area composed of the TT triangles inside $C_{i}$ and those across the border of $C_{i}$.

Figure 4.1 illustrates the relation between $\mathcal{P}_{i}, C_{i}$ and $\mathcal{H}_{i}$ in a summarized form. Observe Figure 4.2 that shows $\mathcal{P}_{i}$ and $C_{i}$ for $i=14$ and $15 . \mathcal{P}_{i}$ is constituted exactly by the vertices that are located on the border of $C_{i}$ as well as those that reside outside $C_{i}$ and comprise the TT triangles crossing the border of $C_{i}$. Hence, by definition, we have the following lemma:

Lemma 4.1. In $G_{T T}$, a vertex is located on $\mathcal{P}_{i}$ if and only if it itself does not resides inside $C_{i}$ and at least one of its neighboring vertex lies inside $C_{i}$.

According to Section 3.5.2, the total number of vertices enclosed inclusively by $\mathcal{P}_{i}$ is exactly $\mu(i)$ (Equation 3.5). 


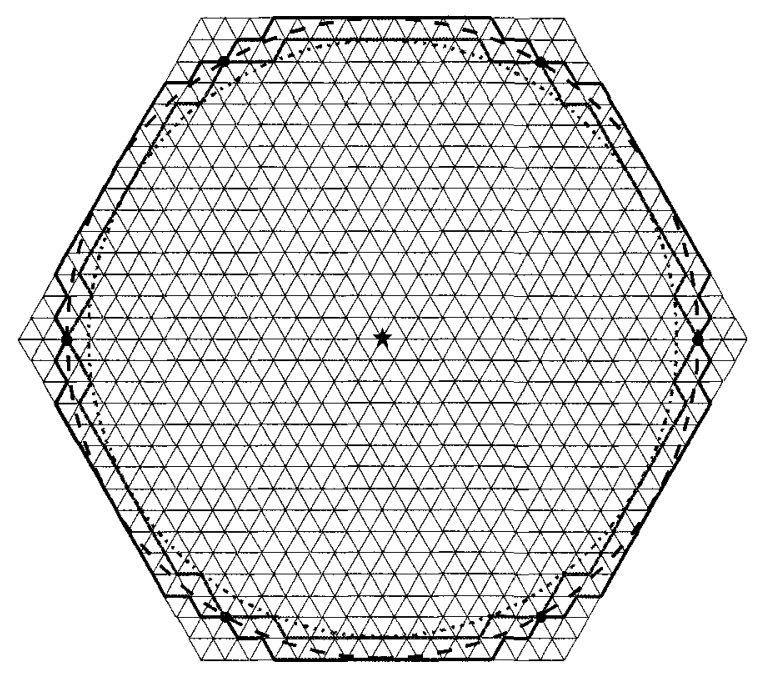

Figure 4.2: Deployment polygons $\mathcal{P}_{14}$ and $\mathcal{P}_{15}$

\subsubsection{Properties}

The six vertex neighbors of any vertex $v$ are all located on a circle centered at $v$. The radius of this circle is equal to TT edge length $l_{e}$. In order for $v$ to be located on $\mathcal{P}_{i}$, this circle must intersect $C_{i}$. It is clear that the radius of $\mathcal{C}_{i}$ is equal to $i h$, where $h=\frac{3}{2} r_{s}$ is the height of a TT triangle. Denote by $\operatorname{Dist}_{E}(v, \Pi)$ the Euclidean distance from $v$ to $\Pi$. Hence, $v$ can be located only on such $\mathcal{P}_{i}$ that $\operatorname{Dist}_{E}(v, \Pi) \leq i h+l_{e}$. It can be trivially derived that a satisfactory $i$ is

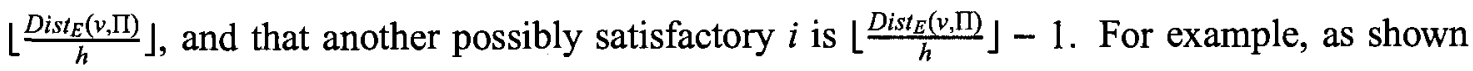
in Figure 4.2 , the vertices marked by solid dots are residing on both $\mathcal{P}_{15}$ and $\mathcal{P}_{14}$. Recall $l_{e}=\sqrt{3} r_{s}$. To sum up, we have the following theorem:

Theorem 4.1 (Residence). In $G_{T T}$, a vertex $v$ must reside on $\mathcal{P}_{i}$ where $i=\left\lfloor\frac{2 D i s t_{E}(v, \Pi)}{3 r_{s}}\right\rfloor$; it will also reside on $\mathcal{P}_{i-1}$ if and only if it has a neighboring vertex $w$ such that $\operatorname{Dist}_{E}(w, \Pi)<$ $\frac{3}{2}(i-1) r_{s}$

Lemma 4.2. In $G_{T T}$, no edge $\overline{u v}$ is shared by two different deployment polygons $\mathcal{P}_{i}$ and $\mathcal{P}_{j}$ $(j<i)$.

Proof. Assume for the sake of contradiction that $\overline{u v}$ is part of $\mathcal{P}_{i}$ and $\mathcal{P}_{j}$. Let $w$ be the common vertex neighbor of $u$ and $v$ that resides on the same side of $\overline{u v}$ as $\mathcal{C}_{i}$. By Lemma 4.1, $u$ and $v$ 


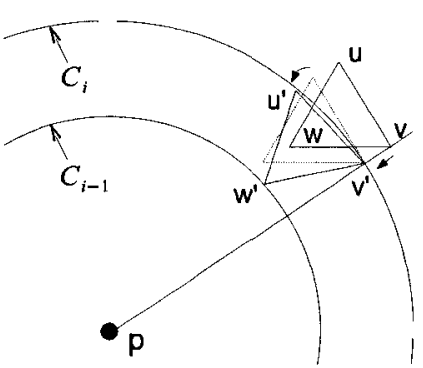

(a) Lemma 4.2

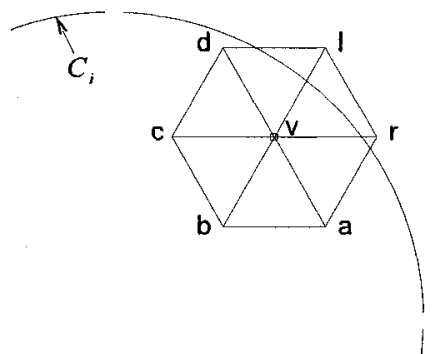

(b) Lemma 4.3

Figure 4.3: Pictures for proving Lemmas 4.2 and 4.3

must not lie inside $C_{i}$; on the contrary, $w$ must be located inside $C_{j}$ (thus inside $C_{i}$ ), namely, $\operatorname{Dist}_{E}(w, \Pi)<R\left(C_{j}\right)$.

Translate $\Delta u v w$ to $\Delta u^{\prime} v^{\prime} w^{\prime}$, as shown in Figure 4.3(a), such that $u^{\prime}$ and $v^{\prime}$ are both located on $C_{i}$ and that $w^{\prime}$ is located inside $C_{i}$. In this case, $w^{\prime}$ is actually located on $C_{i-1}$, i.e., $\operatorname{Dist}_{E}\left(w^{\prime}, \Pi\right)=R\left(C_{i-1}\right) \geq R\left(C_{j}\right)$; apparently, $\operatorname{Dist}_{E}\left(w^{\prime}, \Pi\right) \leq \operatorname{Dist}_{E}(w, \Pi)$. Thus, we have $\operatorname{Dist}_{E}(w, \Pi) \geq R\left(C_{j}\right)$, which contradicts our previously derived result.

Lemma 4.3. On $\mathcal{P}_{i}$, the two vertex neighbors of any vertex $v$ can not be adjacent to each other. Proof. Because $v$ resides on $\mathcal{P}_{i}$, it can not lie inside $\mathcal{C}_{i}$ by Lemma 4.1. Denote the 6 vertex neighbors of $v$ respectively by $a, b, c, d, l$ and $r$. Further, let $l$ and $r$ be the two located on $\mathcal{P}_{i}$. Assume for the sake of contradiction that $l$ and $r$ are adjacent to each other, as shown in Figure 4.3(b). By Lemma 4.1, at least one of the four vertices $a, b, c$ and $d$ lies inside $C_{i}$. Let $a$ be that vertex. Then $b$ must be inside $C_{i}$, because, otherwise, $b$ will be on $\mathcal{P}_{i}$ as well, which is not possible. Since $b$ is inside $C_{i}, c$ must be inside $C_{i}$ for the same reason. In this way, every vertex neighbor of $v$ other than $l$ and $r$ is inside $C_{i}$. Then we reach a contradictory result: $v$ itself must be located inside $C_{i}$ too. This completes the proof.

According to Lemmas 4.2 and 4.3 and through exhaustive enumeration, we derive the following theorem:

Theorem 4.2 (Neighborhood Pattern). In $G_{T T}$, a non-POI vertex has four and only four possible neighborhood patterns, with respect to its residence polygons: 


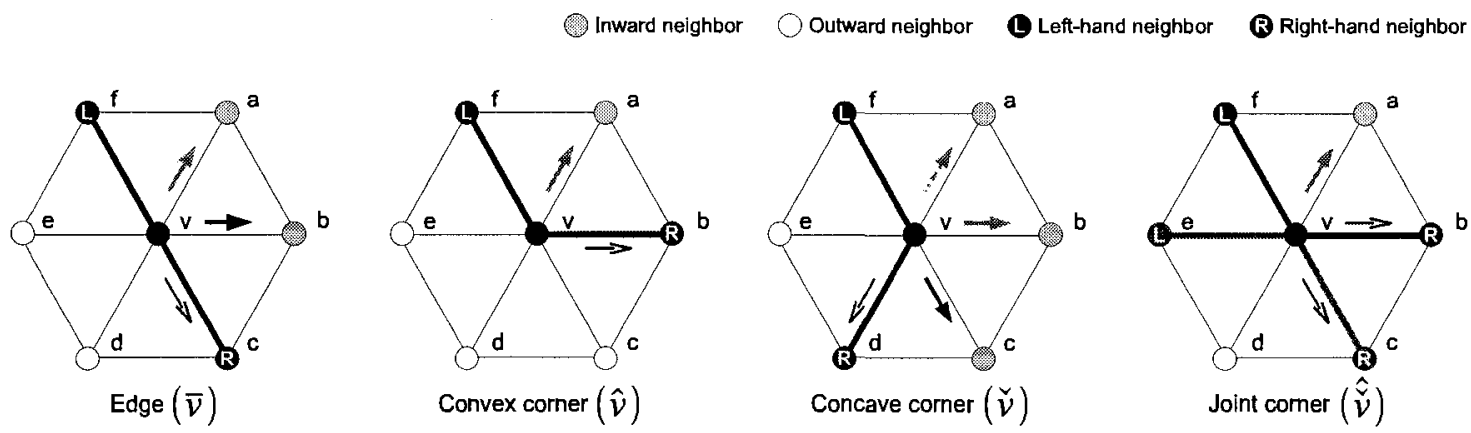

Figure 4.4: Neighborhood patterns of vertex $v$

(1) edge (-): it has one residence polygon and is located on a polygon edge;

(2) convex corner $(\Lambda)$ : it has one residence polygon and is located at a convex corner;

(3) concave corner ( $V$ ): it has one residence polygon and is located at a concave corner;

(4) joint corner $(x)$ : it has two residence polygons and is located at a joint corner.

Figure 4.4 illustrates the four neighborhood patterns. As we can see from this figure, the vertex neighbors of $v$ may be divided into four disjoint sets: left-hand neighbor set $N^{l}(v)$, righthand neighbor set $N^{r}(v)$, inward neighbor set $N^{i}(v)$ and outward neighbor set $N^{o}(v) . N^{l}(v)$ and $N^{r}(v)$ respectively contain the vertex neighbors of $v$ residing on its residence polygons in the clockwise direction and in the counter-clockwise direction around $\Pi ; N^{i}(v)$ and $N^{o}(v)$ respectively contain the vertex neighbors of $v$ residing on an inner deployment polygon and on an outer deployment polygon.

By Theorem 4.1, $v$ must reside on $P_{i}$ where $i=\left\lfloor\frac{2 D i s t_{E}(v, \Pi)}{3 r_{s}}\right\rfloor$. For ease of description, define $i^{\prime}=i-1$ if $P_{i-1}$ is also a residence polygon of $v$, or $i^{\prime}=i$ otherwise. With no difficulty, we can conclude that the vertices in $N^{i}(v)$ are all located on $\mathcal{P}_{i^{\prime}-1}$ and that the vertices in $N^{o}(v)$ are all located on $\mathcal{P}_{i+1}$. Let $\operatorname{Inn}\left(N^{l}(v)\right)$ and $\operatorname{Out}\left(N^{l}(v)\right)$ be the vertex neighbors of $v$ located on $\mathcal{P}_{i^{\prime}-1}$ and $\mathcal{P}_{i+1}$, respectively. Apparently, $\operatorname{Inn}\left(N^{l}(v)\right)=\operatorname{Out}\left(N^{l}(v)\right)$ when $\left|N^{l}(v)\right|=1$.

We denote by $\operatorname{Patt}(v)$ the neighborhood pattern of $v$, and by $\operatorname{Ltm}\left(N^{i}(v)\right), \operatorname{Mid}\left(N^{i}(v)\right)$ and $\operatorname{Rtm}\left(N^{i}(v)\right)$ the leftmost, the middle, and the rightmost vertex neighbors of $v$ in $N^{i}(v)$, respectively. If $\left|N^{i}(v)\right|=2$, then we define $\operatorname{Mid}\left(N^{i}(v)\right)=\operatorname{Rtm}\left(N^{i}(v)\right)$; in the case of $\left|N^{i}(v)\right|=1$, we 
Table 4.2: Neighborhood patterns

\begin{tabular}{|l|l|l|l|l|}
\hline & $\left|N^{l}(v)\right|$ & $\left|N^{r}(v)\right|$ & $\left|N^{i}(v)\right|$ & $\left|N^{o}(v)\right|$ \\
\hline Edge $(-)$ & $1=|\{f\}|$ & $1=|\{c\}|$ & $2=|\{a, b\}|$ & $2=|\{d, e\}|$ \\
\hline Convex corner $(\wedge)$ & $1=|\{f\}|$ & $1=|\{b\}|$ & $1=|\{a\}|$ & $3=|\{c, d, e\}|$ \\
\hline Concave corner $(\vee)$ & $1=|\{f\}|$ & $1=|\{d\}|$ & $3=|\{a, b, c\}|$ & $1=|\{e\}|$ \\
\hline Joint corner $(\times)$ & $2=|\{e, f\}|$ & $2=|\{c, b\}|$ & $1=|\{a\}|$ & $1=|\{d\}|$ \\
\hline
\end{tabular}

define $\operatorname{Ltm}\left(N^{i}(v)\right)=\operatorname{Mid}\left(N^{i}(v)\right)=\operatorname{Rtm}\left(N^{i}(v)\right)$. The above denotations for vertices in $N^{l}(v)$ and $N^{i}(v)$ are likewise applied to those in $N^{r}(v)$ and $N^{o}(v)$ as well.

Table 4.2 lists the size of the four neighbor sets for each neighborhood pattern and also shows their composition based on the vertex notation in Figure 4.4. From this table, a neighborhood pattern is uniquely determined by the combination of any two set sizes (except for the combination of $\left|N^{l}(v)\right|$ and $\left.\left|N^{r}(v)\right|\right)$; by Theorem 4.1, $N^{l}(v), N^{r}(v), N^{i}(v)$ and $N^{o}(v)$ can be computed through trivial computation once the coordinates of $\Pi$ and $v$ are given. Note that the joint corner pattern can in fact be determined directly by Theorem 4.1 .

\subsection{Protocol Description}

In this section, we present an optimized Greedy-Rotation-Greedy $\left(\mathrm{GRG}^{\star}\right)$ algorithm. Unlike GRG, this algorithm uses deployment polygons instead of hexagons to direct node rotation. As deployment polygons best approximate circles in $G_{T T}$ without radius degradation, $\mathrm{GRG}^{\star}$ naturally provides guaranteed circular coverage radius maximization.

$\mathrm{GRG}^{\star}$ adopts the same alignment rule (Rule 3.9) and the same retreat rule (Rule 3.10) as GRG to deal with off-vertex sensors and node collision. It follows the same combined greedyrotation philosophy as GRG for sensors self-deployment. That is, a node located at a vertex $v$ greedily advances to an eligible vertex in $N^{i}(v)$; in the case that no such a vertex exists, it moves to an eligible vertex in $N^{r}(v)$ instead; if no eligible greedy next or rotation next hop exists, it stays still for the time being and resumes deployment movement whenever possible. 
The eligibility of a vertex for deployment next hop is determined by a set of strictly localized hop selection rules. These rules share the same design considerations with their counterparts in GRG; despite design similarity, their definition is not trivial but rather complicated, as polygon vertices have more complex neighbor patterns than hexagon vertices, thus bringing more possibilities and restrictions to hop selection.

In the following, we outline the hop selection rules of $\mathrm{GRG}^{\star}$ in groups in accordance with their purposes. We provide detailed explanation for the rules that differ a lot from their counterparts in GRG; the rules with no or litter change are however presented at short length. As for the alignment rule and the retreat rule, please refer to Section 3.4.4.

\subsubsection{Greedy}

Greedy advance ensures nodal progress toward $\Pi$. It happens to a node when the node proceeds to a vertex from an outward vertex neighbor of the vertex.

The situation that multiple greedily advancing nodes collide at a non-POI vertex $v$ is possible if and only if $\left|N^{o}(v)\right|>1$. According to Table 4.2, this condition is true only in the case $\operatorname{of} \operatorname{Patt}(v)=$ " $" \mid$ " $\wedge$.

Examine the corresponding graphs in Figure 4.4. If two nodes are greedily moving to $v$ from vertices $e$ and $d$ in parallel, they may collide at $v$. But this situation can be locally avoided by the following priority rule as $e$ and $d$ are adjacent to each other.

Rule 4.1 (Priority rule). If two nodes are both aiming at a non-POI vertex $v$ from two different vertices $\operatorname{Ltm}\left(N^{o}(v)\right)$ and $\operatorname{Mid}\left(N^{o}(v)\right)$, the one located at $\operatorname{Mid}\left(N^{o}(v)\right)$ has higher priority than the other.

Note that, in the case of $\operatorname{Patt}(v)=$ " $\wedge$ ", if the two nodes are instead from $e$ and $c$, they could also collide at $v$. To prevent this greedy collision from happening, the following forbiddance rule can be used.

Rule 4.2 (Forbiddance rule). A node that is located at $\operatorname{Rtm}\left(N^{o}(v)\right)$ does not take vertex $v$ as greedy next hop in the case of Patt $(v)=" \wedge "$. 
As a special vertex, $\Pi$ has 6 many outward vertex neighbors. Its occupancy is ensured by the following innermost-layer rule.

Rule 4.3 (Innermost-layer rule). A node located at a vertex on $\mathcal{P}_{1}$ (i.e., $\mathcal{H}_{1}$ ) moves to $\Pi$ as long as $\Pi$ is to its knowledge not occupied.

The innermost-layer rule may induce node collision at $\Pi$, but fortunately no more than once according to Section 3.4.1. As in GRG, this potential greedy collision can be avoided by a gateway rule to be introduced later, in Section 4.4.3.

\subsubsection{Rotation}

Rotation resumes blocked greedy advance by guiding nodes around occupied vertices. It happens to a node when the node moves to a vertex from the vertex's left-hand vertex neighbor.

Again examine Figure 4.4. If a node is moving to $v$ from $f$ while another node is proceeding to $v$ from $e$, the two nodes are likely to collide at $v$. This potential collision, called rotation collision if $\operatorname{Pat}(v)=$ " $\times$ " or greedy-rotation collision otherwise, can be prevented by the following competition rule.

Rule 4.4 (Competition rule). If two nodes are competing for vertex $v$ from two different vertices $\operatorname{Out}\left(N^{l}(v)\right)$ and $\operatorname{Ltm}\left(N^{o}(v)\right)$ (or, $\operatorname{Inn}\left(N^{l}(v)\right)$ and $\left.\operatorname{Out}\left(N^{l}(v)\right)\right)$, the one from $\operatorname{Out}\left(N^{l}(v)\right)$ (resp., $\left.\operatorname{Inn}\left(N^{l}(v)\right)\right)$ wins.

If the two nodes are instead from $a$ and an outward vertex neighbor, different than $e$, of $v$, they could also collide at $v$. But this collision is no longer avoidable by the above rule. We will elaborate and resolve this situation later, in Section 4.4.3.

Figure 4.4 implies that $\operatorname{Rtm}\left(N^{o}(v)\right)$ is always adjacent to $\operatorname{Out}\left(N^{r}(v)\right)$. If a node located at $v$ discovers that some node is rotating to $\operatorname{Rtm}\left(N^{o}(v)\right)$, then it knows that the node will proceed to $\operatorname{Out}\left(N^{r}(v)\right)$ if it itself does not chose $\operatorname{Out}\left(N^{r}(v)\right)$ as rotation next hop. This is the design consideration of the following suspension rule, which prevents rotation loop and ensures nodes' greedy advance, as analyzed in Section 3.4.2. 
Rule 4.5 (Suspension rule). A node located at vertex $v$ does not rotate to $\operatorname{Out}\left(N^{r}(v)\right)$ if any of its neighbors is currently rotating to $\operatorname{Rtm}\left(N^{o}(v)\right)$.

Note that, when the collision avoidance rules (to be defined in Section 4.4.3), are applied, if greedy advance at $\operatorname{Rtm}\left(N^{o}(v)\right)$ is not allowed by those rules, the above suspension rule should be ignored, since in this case rotation suspension does not make any sense and might even cause sensing holes.

\subsubsection{Collision avoidance}

The greedy rules preclude non-POI-based greedy collision (i.e., collision between greedily moving nodes) but leave the POI-based still possible. The rotation rules prohibit rotationrotation collision completely but eliminate greedy-rotation collision only in part. It is because they rely on the adjacency of the greedy prior hop and the rotation prior hop of a vertex, which however does not always remain. Below, we will discuss how to totally preclude these collisions.

We start with the easiest part, i.e., enabling $\mathrm{GRG}^{\star}$ to generate no greedy collision at $\Pi$. This goal can be accomplished simply by the following gateway rule of GRG, which serves as the replacement of the innermost-layer rule.

Rule 4.6 (Gateway rule). $A$ vertex on $\mathcal{P}_{1}$ (i.e., $\mathcal{H}_{1}$ ) is pre-defined as the gateway to $\Pi$. For $a$ node located on $\mathcal{P}_{1}$, it performs only greedy advance if its home vertex is the gateway, or only rotation otherwise.

In the rest of this section, we will focus on greedy-rotation collision avoidance. For ease of description, we say a node's greedy advance is "safe" if and only if the movement will cause no greedy-rotation collision,

Undoubtedly, in order not to generate greedy-rotation collision, a node must not perform greedy advance unless it knows the movement is definitely safe. From a localized perspective, a node is able to make such an assurance if and only if all the left-hand vertex neighbors of the greedy next hop is shared or neighbored by the home vertex of the node. 


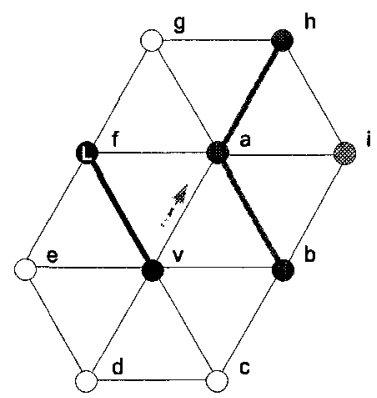

(a) $\operatorname{Patt}(a)=" \wedge "($ case 1$)$

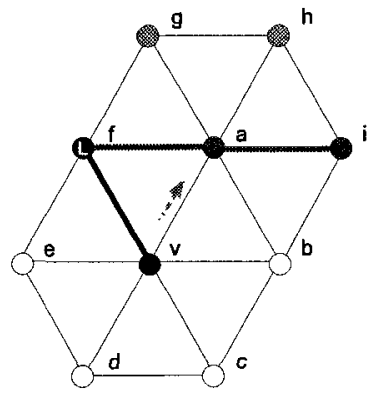

(d) $\operatorname{Patt}(a)="-"($ case 2)

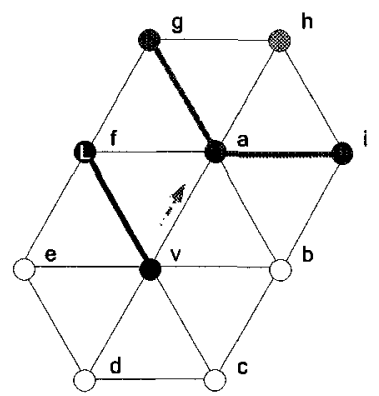

(b) Patt (a) $=" \wedge "($ case 2$)$

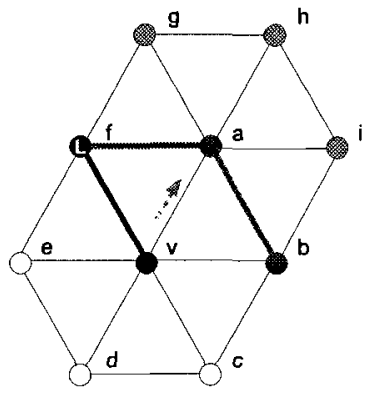

(e) $\operatorname{Patt}(a)=“ \vee "$

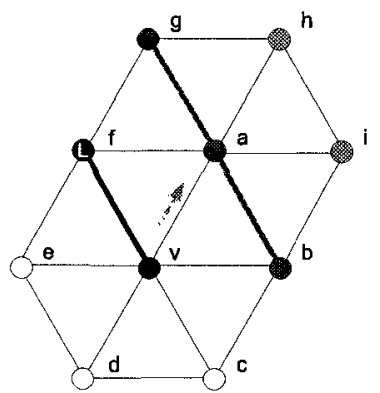

(c) $\operatorname{Patt}(a)="-"($ case 1$)$

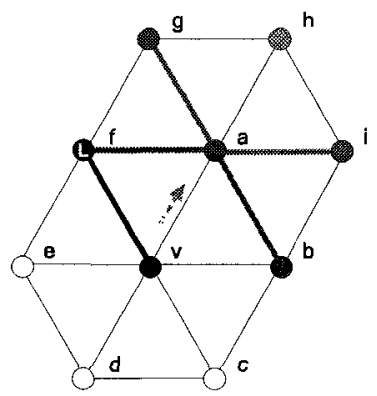

(f) $\operatorname{Patt}(a)=" x "$

Figure 4.5: The leftmost inward vertex neighbor $a$ of $v$

Consider a vertex $v$ on $\mathcal{P}_{i}$ for $i>1$. Suppose that a node is located at $v$ and that it has decided by the greedy rules to move to $\operatorname{Ltm}\left(N^{i}(v)\right)$. Figure 4.5, where $a=\operatorname{Ltm}\left(N^{i}(v)\right)$ and $f=\operatorname{Inn}\left(N^{l}(v)\right)$, enumerates all the possible scenarios with respect to $\operatorname{Patt}(a)$.

In the case of $\operatorname{Patt}(a)=" \wedge "$ (where $\left.\operatorname{Out}\left(N^{l}(a)\right)=\operatorname{Inn}\left(N^{l}(a)\right)\right)$, there are two possible scenarios, i.e., $v=\operatorname{Rtm}\left(N^{o}(a)\right)$ or $v=\operatorname{Mid}\left(N^{o}(a)\right)$, as illustrated in Figure 4.5(a) and 4.5(b). In the first scenario, whatever Patt(v) is, the node is not allowed to move to $a$ by the forbiddance rule. This scenario is then out of our consideration. Examine the second scenario, where Patt $(v)$ must be either " $\wedge$ " (defined by $f$ and $b$ ) or " $\times$ " (defined by $f, b, e$ and $c$ ). As we see $v$ is not adjacent to $\operatorname{Out}\left(N^{l}(a)\right)$, the node's movement is not safe.

In the case of $\operatorname{Patt}(a)=$ " $" ~\left(\right.$ where $\left.\operatorname{Out}\left(N^{l}(a)\right)=\operatorname{Inn}\left(N^{l}(a)\right)\right)$, there are also two possible scenarios. One, as shown in Figure 4.5(c), is that $v=\operatorname{Rtm}\left(N^{o}(a)\right)$. In this scenario, $v$ is not adjacent to $\operatorname{Out}\left(N^{l}(a)\right)$ (i.e., g), although Patt(v) can be any of " - " (defined by $f$ and $c$ ), " $\vee$ " (defined by $f$ and $d$ ), " $\wedge$ " and " $\times$ ", implying that the node's greedy advance is not safe. The 
other is that $v=\operatorname{Ltm}\left(N^{o}(a)\right)$. In this scenario, Patt $(v)=" \wedge " \mid " \times "$, and the node's greedy advance is always safe since $\operatorname{Inn}\left(N^{l}(v)\right)=\operatorname{Out}\left(N^{l}(a)\right)=f$.

If $\operatorname{Patt}(a)=" \vee " \mid " \times "$, then $\operatorname{Inn}\left(N^{l}(v)\right)=\operatorname{Out}\left(N^{l}(a)\right)=f$ and $\operatorname{Patt}(v)=“-" \mid$ " $\wedge " \mid " \times "$. Notice that $\operatorname{Patt}(v) \neq$ " $\vee "$. In the case of $\operatorname{Patt}(a)=$ " $\vee "$, which is depicted in Figure 4.5(e), the node can safely advance to $a$ since $\operatorname{Inn}\left(N^{l}(v)\right)=\operatorname{Out}\left(N^{l}(a)\right)$, just similar to the scenario given in Figure 4.5(d); whereas, in the case of Patt $(a)=$ " $\times$ ", which is displayed in Figure 4.5(f), it can not, since $v$ is not adjacent to $\operatorname{Inn}\left(N^{l}(a)\right.$ ) (i.e., $\left.g\right)$ and $\operatorname{Inn}\left(N^{l}(a)\right) \neq \operatorname{Out}\left(N^{l}(a)\right.$ ).

When $\operatorname{Patt}(v)=$ " "|“ $\vee "$, as we can see from Figure 4.4,b=Mid(Ni(v)) and $a=$ Out $\left(N^{l}(b)\right)$. Assume that a node located at $v$ has determined to move to $b$ by the greedy rules. If $\operatorname{Out}\left(N^{l}(b)\right)=\operatorname{Inn}\left(N^{l}(b)\right)$, namely, if Patt $(b) \neq$ “ $\times ”$, the node's movement will be safe; otherwise, it is "dangerous" as the node may possibly collide with some unknown node moving to $b$ from $\operatorname{Inn}\left(N^{l}(b)\right)$ that $v$ is not adjacent to.

In the case of $\operatorname{Patt}(v)=$ " $\vee ", v$ has yet one more inward vertex neighbor $c$ in addition to $a$ and $b$. If the node decided to move to $c$ instead of $b$, it may execute the movement safely, because $b$ must be the only left-hand vertex neighbor of $c$.

According to the above analysis, we define the following three collision avoidance rules:

Rule 4.7 (Edge rule). A node located at vertex $v$ with Pat $(v)=$ " - " does not take $\operatorname{Ltm}\left(N^{i}(v)\right)$ $\left(\operatorname{or} \operatorname{Rtm}\left(N^{i}(v)\right)\right)$ as greedy next hop if $\operatorname{Pat}\left(\operatorname{Ltm}\left(N^{i}(v)\right)\right)=$ " $-" \mid “ \times ”\left(\operatorname{resp} ., \operatorname{Pat}\left(\operatorname{Rtm}\left(N^{i}(v)\right)\right)=\right.$ " $\times$ ").

Rule 4.8 (Concave-corner rule). A node located at vertex $v$ with Pat $(v)=$ " $\vee "$ does not move to $\operatorname{Ltm}\left(N^{i}(v)\right)\left(\right.$ or $\left.\operatorname{Mid}\left(N^{i}(v)\right)\right)$ if $\operatorname{Pat}\left(\operatorname{Ltm}\left(N^{i}(v)\right)\right)=$ " - " (resp., “×”).

Rule 4.9 (Convex-corner rule). A node located at vertex $v$ with Pat $(v)=$ " $\wedge " \mid$ " $\times$ " does not move to $\operatorname{Ltm}\left(N^{i}(v)\right)$ if $\operatorname{Pat}\left(\operatorname{Ltm}\left(N^{i}(v)\right)\right)=" \wedge " \mid$ " $\times " \mid$ " $"$, where " - " additionally requires $\left.\operatorname{Out}\left(N^{l}\left(\operatorname{Ltm}\left(N^{i}(v)\right)\right)\right) \neq \operatorname{Inn}\left(N^{l}(v)\right)\right)$.

We would like to indicate that, $\mathrm{GRG}^{\star}$ is not collision free in general as GRG largely due to stochastic node distribution. If node were initially place at distinct vertices, GRG^ would generate no collision at all after the above four collision avoidance rules are applied. 


\subsection{Performance evaluation}

In this section, we shall present our comparative simulation study on GRG and GRG ${ }^{\star}$. Because the two algorithms follow the same self-deployment strategy, they have similar performance in general, which can be clearly seen from the same shape of their performance curves in Figures 4.7(a) - 4.7(f). As our simulation results are consistent with those detailedly presented in Section 3.6.3, here we emphasize on the difference between GRG and GRG only.

Through simulation we wish to study the performance difference of GRG and GRG ${ }^{\star}$ for achieving the same coverage radius $\gamma^{C}$, with proper network size $n$. We chose the following evaluation metrics, which are also adopted in Chapter 3:

- Convergence Time (CT): the number of time units that it takes the network to stabilize (have no floating node);

- Number of Collisions (NC): the number of times that node collision occurs during the course of deployment.

- Progress (PG): the average progress a node makes toward $\Pi$ during its self-deployment process.

- Mileage (MG): the average distance that a node travels during the course of its selfdeployment;

- Mileage over Progress (MP): the ratio MG over PG, which gives an idea about how costly zigzag node movement is.

- Number of Moves (NM): the average number of times that a node changes its status from still to moving; 


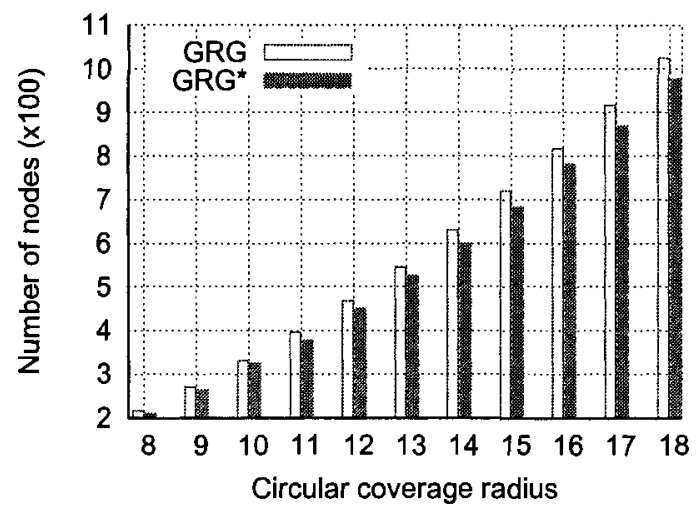

Figure 4.6: Number of nodes (nN) v.s. Coverage radius (cR)

\subsubsection{Simulation setup}

We implemented GRG and GRG ${ }^{\star}$ within a custom network simulator, and simulated their execution over a mobile sensor network randomly and uniformly dropped in two-dimension free plane. The geographic center of the dropping area is taken as Point of Interest (POI), denoted by $\Pi$. Nodes have sensing radius $r_{s}=10$ and communication radius $r_{c}=\sqrt{3} r_{s}=$ $\sqrt{3} \times 10 \approx 18$; they may move at different speeds, ranging from 0.05 to 0.2 per simulated time unit, for their every single step.

In simulation, we fixed the size of dropping area to $500^{2}$ and target at different coverage radius $\gamma^{C}$ by varying network size $n$ properly. For a $\gamma^{C}$ less than $8, G^{\star} G^{\star}$ and GRG produce exactly the same focused coverage according to Equation 3.5. Thus we considered the cases of $\gamma^{C}=8, \ldots, 18$. Figure 4.6 shows $n$ as a function of $\gamma^{C}$; it is generated using Equations 3.1 and 3.5. By using fixed dropping area and varied $n$, we were able to study the impact of node density on algorithm performance.

In order to minimize data noises, for each simulation setting, we executed GRG and GRG ${ }^{\star}$ over 50 randomly generated network scenarios and computed average results. 


\subsubsection{Experimental results}

\section{Convergence and collision}

Let us first examine the convergence time (CT) and the number of collision (NC) of GRG and $\mathrm{GRG}^{\star}$. As we will see, $\mathrm{GRG}^{\star}$ has better performance than GRG in these two aspects, especially in dense networks.

Since $\mathrm{GRG}^{\star}$ uses less nodes than GRG to achieve the same $\gamma^{C}$ (specifically, when $\gamma^{C}$ is larger than 7), it should naturally spend less number of time units stabilizing the network, i.e., to converge, than GRG. This is indeed true as confirmed by Figure 4.7(a), from which we can see the curves of GRG ${ }^{\star}$ always stays below those of GRG. Notice that the gaps between these curves intend to be bigger and bigger after $\gamma^{C}$ is beyond number 13. Later we will see that this "magic" $\gamma^{C}$ value is the one that leads to the lowest PG in GRG in magnitude.

Figure 4.7(b) depicts $\mathrm{NC}$ as a result of $\gamma^{C}$. It is observed that GRG always generates a larger NC than $\mathrm{GRG}^{\star}$. This difference is rooted at the fact that GRG requires more nodes than GRG for achieving the same $\gamma^{C}$. Since the dropping area is fixed, the more nodes, the larger node density, and thus the more often node collision occurs. According to Figure 4.6, the two algorithms' difference on required network size $n$ actually becomes bigger and bigger as $\gamma^{C}$ goes up. This change is reflected by the growing gap between the NC curves of GRG and $\mathrm{GRG}^{\star}$ in Figure 4.7(b).

\section{Energy consumption}

We shall now study the energy consumption of GRG* in comparison with GRG. As we will see, GRG ${ }^{\star}$ consumes slightly more energy in sparse networks, but much less energy in dense networks, than GRG.

Let $D_{i n i}$ and $D_{f i n}$ be the average node initial distance and average node final distance to $\Pi$, respectively. Then PG is equal to $D_{i n i}-D_{f i n}$. Because nodes are randomly and uniformly placed at initiation, $D_{i n i}$ is approximately a constant value; whereas, $D_{f i n}$ ever increases as $\gamma^{C}$ goes up, regardless of initial node placement. In this case, PG is expected to be a monotoni- 


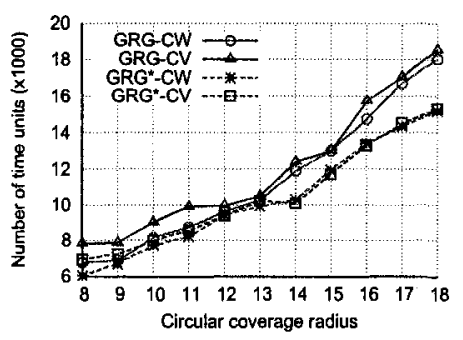

(a) Convergence Time (CT)

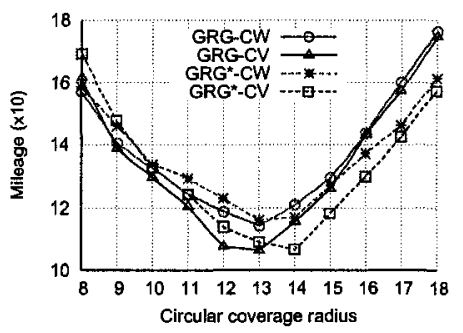

(d) Mileage (MG)

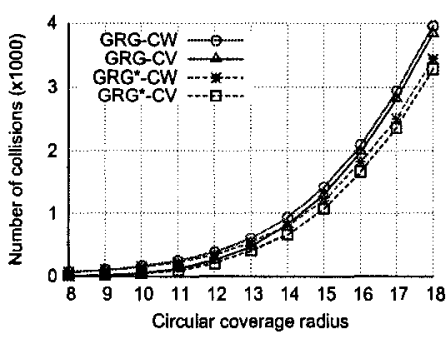

(b) Number of Collisions (NC)

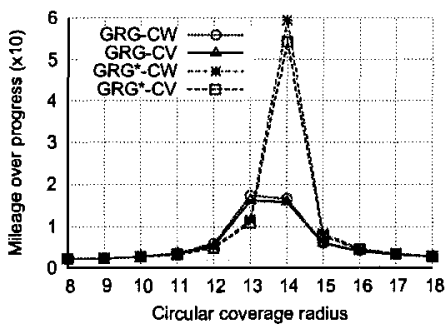

(e) Mileage over Progress (MP)

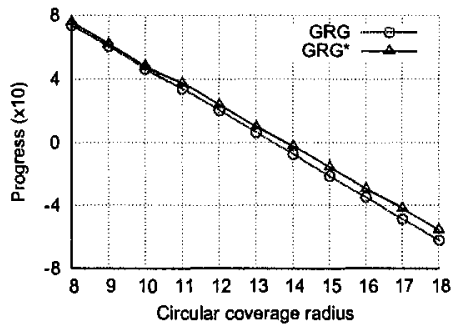

(c) Progress (PG)

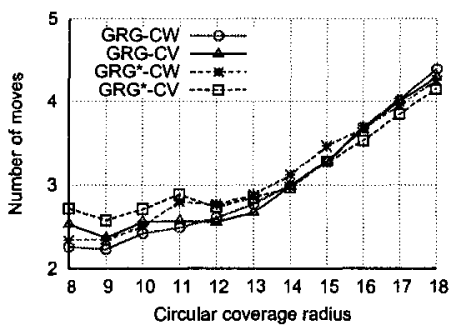

(f) Number of Moves (NM)

Figure 4.7: Experimental results

cally decreasing function of $\gamma^{C}$.

When node density is low, nodes intend to move toward $\Pi$, i.e., $D_{i n i}>D_{\text {fin }}$, and thus PG has a positive value. As the network becomes increasingly dense, $D_{f i n}$ approaches to $D_{i n i}$, and PG gradually drops to 0 as a result. In this case, the network exhibits a rotating behavior. As node density continues to increases, the network eventually show an expanding behavior since nodes try to move outwards, rendering PG negative.

The above expectations about PG have been confirmed in Section 3.6.3 and are once again validated by our experiments as shown in Figure 4.7(c). Observe this figure and notice the difference between GRG and $\mathrm{GRG}^{\star}$ : the curve of $\mathrm{GRG}^{\star}$ is always above that of GRG. It is because of the larger $D_{f i n}$ of GRG, which is in turn due to the coverage redundancy of GRG.

Figure 4.7(d) shows the difference between GRG and GRG ${ }^{\star}$ in MG. Observe that MG reaches the lowest value later in $\mathrm{GRG}^{\star}$ than in GRG. It is because $\mathrm{GRG}^{\star}$ uses less nodes than GRG for achieving the same $\gamma^{C}$, delaying the behavioral change of the network. From this figure, it is additionally observed that, $\mathrm{GRG}^{\star}$ has slightly larger (or much smaller) $\mathrm{MG}$ than that of GRG in concentrating (resp., expanding) network. 
In both GRG and $\mathrm{GRG}^{\star}$, nodes move along curly path to their final position; thus their MG is usually larger than their PG. The ratio of Mileage over Progress (MP) gives an idea about how costly zigzag node movement is. From Figure 4.7(e), MP is most of time below 5; it increases dramatically only when $\gamma^{C}$ is around the value leading to lowest PG in magnitude (see Figure 4.7(c)), although MG approaches the lowest value at the same time (see Figure 4.7(d)). Again, as shown in the figure, the economic node usage of $\mathrm{GRG}^{\star}$ delays the appearance of the peak value of its MP; GRG and GRG ${ }^{\star}$ have nearly the same MP performance in the other cases.

Nodes in $\mathrm{GRG}^{\star}$ are regulated by more hop selection rules and have to stop moving more often than in GRG. On the other hand, GRG requires more nodes than $\mathrm{GRG}^{\star}$ for achieving the same $\gamma^{C}$ and therefore possibly causes relatively frequent node blocking. As confirmed by Figure 4.7(f), when $\gamma^{C}$ is not too large compared with the dropping area, in other words, when node density is moderate, the impact of hop selection rules dominate algorithm performance on NM, making GRG generate less NM than $\mathrm{GRG}^{\star}$; but it is slowly overwhelmed by the latter as $\gamma^{C}$ becomes increasingly large, rendering $\mathrm{GRG}^{\star}$ eventually overtake GRG.

\subsection{Summary}

Focused coverage formation is a new sensor self-deployment introduced in the previous chapter. The solution protocol Greedy-Rotation-Greedy (GRG) proposed earlier can provide hexagonal coverage guarantee. But its resulting circular coverage radius is not optimal event though it approaches the optimal value in a factor close to 1 .

In this chapter, we optimized GRG to enable it to guarantee circular coverage radius maximization. The optimized version of GRG is called GRG* ${ }^{\star}$. It follows the same greedy-rotation idea for sensor self-deployment as GRG. But nevertheless, unlike GRG which guides sensors to rotate along hexagons, $\mathrm{GRG}^{\star}$ direct sensors to rotate along our so called deployment polygons that approximate circles best in TT graph without radius reduction. The use of de- 
ployment polygons enable OGRG to always produce maximized circular coverage radius. We evaluate OGRG in comparison with GRG through extensive set of simulation. Our study show that OGRG has better performance than GRG in many aspects. 


\title{
CHAPTER 5
}

\section{DISTANCE-SENSITIVE SERVICE DISCOVERY}

\begin{abstract}
s hardware technology advances, WSNs are evolving toward service-oriented robotic 1 networks such as MSNs and WSANs, where mobile/robotic service providers offer services, via geographic relocation, to other nodes or to the physical world. This evolution necessitates the research of distance-sensitive service discovery, where a solution protocol is expected to provide closest/nearby selection guarantee. Existing generic service discovery algorithms and adoptable techniques are developed without distance sensitivity in mind. They are not a practical candidate for their large overhead in communication and/or in storage. In this chapter, we for the first time formalize the distance-sensitive service discovery problem, and propose a purely localized algorithm, iMesh.
\end{abstract}

In Section 5.1, we give the problem formalization and a brief description of iMesh; in Section 5.2, we define the network model; in Section 5.3, we present iMesh in detail and study its properties including message complexity, storage load and distance sensitivity in grid sensor networks; in Section 5.4, we show how to implement iMesh in arbitrary sensor networks; in Section 5.5, we evaluate iMesh in comparison with Quorum $[48,72,74]$ through an extensive set of simulation; in Section 5.6, we summarize the chapter. 


\subsection{Introduction}

A WSN is a collection of micro-sized wireless sensing devices, sensors, deployed in a region of interest for object monitoring and/or target tracking. Traditionally, sensor nodes are responsible only for sampling their surroundings and reporting to a pre-defined data sink. As hardware technology advances, WSNs are now evolving toward service-oriented networks such as MSN $[1,20]$ and WSAN $[6,56,57]$, where some service providers, i.e., mobile sensors or actors, offer movement-assisted services to other nodes and/or to the physical world. In MSNs, redundant sensors can geographically relocate to replace failed ones; in WSANs, actors may move to a target location to, for example, repair faulty sensors, turn off water tap in cast of water-overflow, extinguish a fire in a woody area, interact with patients in a health care system, rescue survivors in an emergency situation, etc.

Service discovery is a crucial component of any service-oriented network. Discovery criteria depend on the underlying network and the application. In the above movement-assisted service delivery cases, delivery distance is a primary concern for energy-saving and timely response. Moreover, since we are in the context of WSNs with various resource constraints, service discovery must be performed in an efficient way, i.e., with constant storage load on each node and with no global computation. Although many generic service discovery algorithms $[24,31,35,39-41]$ and adoptable techniques $[3,22,44,46,65,68,74]$ have been proposed for wireless ad hoc networks, they have major weaknesses. In particular, they are not suitable for the problem of distance-sensitive service discovery in resource-constrained WSNs, where the algorithms are expected to provide closest/nearby service selection guarantee.

Definition 5.1 (Closest service selection guarantee). Denote by $P$ the set of service providers and by $C$ the set of service consumers. $\forall a \in C,|a t|=\min _{p \in P}\{|a p|\}$, where $t$ is the service provider discovered by $a$.

Definition 5.2 (Nearby service selection guarantee). Denote by $P$ the set of service providers and by $C$ the set of service consumers. $\forall a \in C,|a t| \leq 2 \times \min _{p \in P}\{|a p|\}$, where $t$ is the service 
provider discovered by $a$.

Intuitively, if we construct a Voronoi diagram [9] using service providers as creating points and let each of them distribute its location information along the perimeter of its Voronoi polygon, then the Voronoi diagram becomes a distributed service directory with bounded pernode storage load. In this case, distance-sensitive service lookup becomes localized (restricted within its Voronoi polygon): a service consumer queries along a path in an arbitrary direction, and will find its closest service provider once it hits the perimeter of its home Voronoi polygon. This intuitive solution possesses all the properties that we are looking for, but it requires global computation. Hence, to make this solution practical, as service directory we must substitute the Voronoi diagram with a localized planar structure that has good proximity.

A naive idea of improvement is to replace Voronoi digram with square mesh constructed by the well-known quorum based location service (Quorum for short) $[48,72,74]$. That is, service providers propagate their location information horizontally and vertically across the entire network; the propagation paths form a mesh structure as service directory. Although this method requires only local computation, it can generate inconstant storage load on network nodes, for example, if service providers are all placed in a line, and it also makes the localized in-cell lookup no longer able to provide closest/nearby service selection guarantee because the mesh structure bears no proximity property.

As we show in this chapter, it is however possible to modify the quorum technique to obtain a planar structure that (as the mesh but unlike the Voronoi diagrams) can be constructed in a purely localized manner and (as the Voronoi diagram but unlike the mesh) possesses our required guaranteed proximity property. The needed modification is the use of distance-based blocking. It has been informally suggested by Tchakarov and Vaidya [77] for a different problem of content location service, and is formally rediscovered by us in this thesis work. Unlike the protocol proposed here, their proposal did not contain any (correct) theoretical analysis of the resulting information structure, and it did not consider the use, in addition to blocking, of an important extension rule that we show leads to major improvements in the 
performance.

\subsubsection{Our contributions}

We propose a novel localized distance-sensitive service discovery protocol, $i M e s h$, for WSNs. The proposed protocol is based on the planar structure, which we refer to as information mesh, created by the use of an existing blocking rule [77] in the traditional quorum-based mesh construction, and the use of a newly proposed information extension rule. In iMesh, service providers publish their location information in four directions: north, west, south and east. During their transmission, these information collinearly or orthogonally block each other according to the blocking rule: a node receiving information from multiple service providers forwards only the information of the closest one. They may however also be extended to other directions by the extension rule: a node where information $x$ orthogonally blocks information $y$ transmits $x$ along the backward transmission path of $y$ for a limited distance. Information propagation paths together constitute the information mesh that distributedly stores the locations of all the service providers. To discover nearby services, service consumers can simply conduct a cross lookup process within their residing mesh cells.

We presents a thorough analytical study as well as a comprehensive simulation study on iMesh. For ease of understanding, the theoretical analysis is conducted over a grid sensor network model. It focuses on the properties (construction cost and distance-sensitivity) of the information structure constructed by the basic version of iMesh (denoted by iMesh-A) using the blocking rule only, and by the complete iMesh (denoted by iMesh-B) containing both the blocking rule and the extension rule. The performance evaluation is provided through extensive simulation of iMesh-A/B and Quorum $[48,72,74]$ in randomly placed sensor networks. Our experimental results show that iMesh generates significantly lower message overhead than Quorum, and that iMesh-A/B guarantee closest service selection respectively with probability $>95 \%$ and $>97 \%$, and nearby service selection both with probability $>99 \%$. They further indicate that iMesh-B noticeably improves the distance sensitivity of iMesh-A with negligible 
extra communication. In summary, our main contributions in this chapter include:

- We formalize the distance-sensitive service discovery problem in WSNs as well as the existing blocking rule for information propagation.

- We propose a novel localized solution algorithm iMesh by combining the blocking rule and the new extension rule with the quorum technique $[48,72,74]$.

- We derive the message complexity, the constant per node storage load property, and the distance sensitivity of iMesh with formal proof of correctness for grid networks.

- We show that the claim, the blocking rule allows a node to discover a service that is at a distance no larger than $\sqrt{2}$ times the distance from the closest [77], is false.

- We evaluate iMesh by extensive simulation on random networks and conclude that iMesh nearly (with very high probability) guarantees nearby service selection.

\subsection{Model and definitions}

We first consider a WSN of unknown size $n$, where nodes are placed exactly at the intersection points of a grid structure. For this model we provide theoretical analysis. We then consider randomized sensor placement in the field and confirm our findings by simulation in this widely used setting, showing practicality of our approach.

Nodes are classified as service providers (SPs) or service consumers (SCs). In practice, SCs may be the nodes that require services themselves or the nodes that require services on behalf of their monitored physical objects. SPs are scattered in the network at random. All the nodes, whether SPs or SCs, have the same communication radius. We denote such a network by $G$. We use $v(G)$ to represent the number of SPs in $G$. By definition, $v(G) \leq n$. Given $G$, $v(G)$ can be written as $v$ without ambiguity.

We require that all the nodes know their own geographic location through a localization system such as the Global Positioning System (GPS) [2]. We believe this requirement is 
Table 5.1: Notations

\begin{tabular}{ll}
\hline$G$ & A network grpah \\
$n$ & Network size; a unknown value \\
$v(G)$ & The number of service providers in $G ;$ simplified as $v$ \\
$b \stackrel{\leftrightarrow}{\rightarrow} a$ & Node $b$ blocks node $a$ at node $u$ \\
$b \stackrel{k}{\Rightarrow} a$ & Node $b$ chain-blocks node $a$ at length $k$ \\
$\mathcal{M}(G)$ & The information mesh constructed over $G ;$ simplified as $\mathcal{M}$ \\
$\eta(\mathcal{M})$ & The extension of $\mathcal{M}$; simplified as $\eta$ \\
$H C e l l(a)$ & The home cell of a service consumer $a$ \\
$T(a)$ & The target service provider of a service consumer $a$ \\
$C(a)$ & The closest service provider of a service consumer $a$ \\
$T C R(a)$ & The target over closet ration of a service consumer $a$ \\
\hline
\end{tabular}

reasonable because of the surveillance goal of WSNs. We assume the standard restrictions, i.e., total reliability, FIFO communication channel, bidirectional links and finite communication delay, which are commonly used in distributed computing domain [67].

The reason for choosing to study first the grid network model is that we want to emphasize on the theoretical aspects of iMesh. In fact, iMesh can be implemented in arbitrary network scenarios by using GFG routing [15,27] as part of Quorum $[48,72,74]$. We then use uniform random sensor networks in our simulation study to confirm theoretical findings.

\subsection{Protocol description}

In this section, we describe iMesh and study its properties over a grid sensor network model. Later in Section 5.4, we will present the implementation details in arbitrary sensor networks.

\subsubsection{Basic iMesh}

In this section, we will present the basic version (or version $\mathbf{A}$ ) of iMesh, $i M e s h-A$. This version uses the blocking rule alone to build the service directory, i.e., information mesh. The 


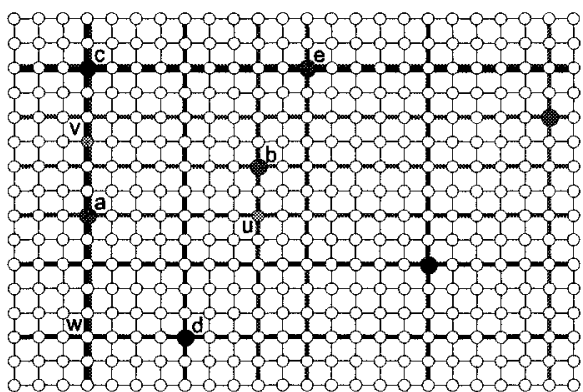

(a) A complete mesh

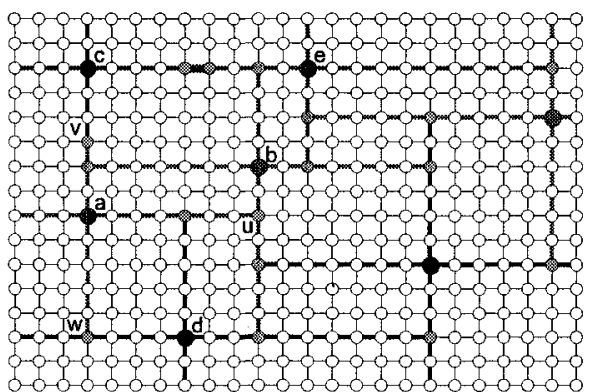

(b) An information mesh

Figure 5.1: Information mesh construction

complete version (or version B) of iMesh, $i$ Mesh- $B$, which contains both the blocking rule and the extension rule, will be presented later, in Section 5.3.2.

\section{Information mesh construction}

Consider only the residing rows and columns of the SP nodes in $G$. They intersect each other and constitute a complete mesh, as illustrated in Figure 5.1(a), where SP nodes are represented by solid big dots, and their residing rows and columns are highlighted by thick lines. If each SP distributes its own location information (by a registration message) among the nodes along its residing row and column, this complete mesh distributedly stores the location information of all the SPs and therefore can be used for the purpose of service discovery.

Let us closely examine the complete mesh structure in Figure 5.1(a). SP node $c$ is closer to the area above the mid-point node $v$ between itself and the vertically collinear SP node $a$, and thus it has relatively high priority to be discovered by the SC nodes in that area. In addition, SP node $b$ might be a better choice for the SC nodes located in its right-side area than SP node $a$. In these cases, $a$ does not need to distribute its location information in those areas. Similar argument can be made again for other SP nodes. According to this observation, we define the following blocking rule, an informal definition of which can also be found in [77].

Rule 5.1 (Blocking Rule - A Formalization). A node u shared by the residing rows/columns of two SPs $a$ and $b(a \neq b)$ stops the further propagation of the information of $a$, iff $|u a|>$ 
$|u b| \vee|u a|=|u b| \wedge$ colline $(a, b) \vee|u a|=|u b| \wedge \neg$ colline $(a, b) \wedge$ horizon $(b)$, where colline $(a, b)$ and horizon $(b)$ denote the case that $a$ and $b$ are (vertically or horizontally) collinear and the case that the involvement of $b$ is along the horizontal direction, respectively. When this blocking happens, we say " $b$ blocks $a$ at $u$ " and denote it by $a \stackrel{u}{\leftarrow} b$ or $b \stackrel{\leftrightarrow}{\rightarrow} a$.

The application of above blocking rule will cause some edges of the complete mesh structure to be removed and therefore the merge of their adjacent mesh cells. The resulting pruned mesh structure is what we call information mesh. We denote the information mesh constructed on top of $G$ by $\mathcal{M}(G)$ (or simply by $\mathcal{M}$ ). Figure 5.1(b), where solid small dots represent the nodes at which the blocking rule is applied, shows the information mesh corresponding to the complete mesh structure in Figure 5.1(a). According to the definition of the blocking rule, we have the following corollary.

Corollary 5.1. $\mathcal{M}$ is a planar structure.

In an asynchronous environment, a node $c$, at which an SP node $b$ is supposed to block another SP node $a$, may wrongly retransmit the registration message of $a$, because of the late arrival of the registration message of $b$, violating the blocking rule. Fortunately, this problematic situation can be identified by $c$, as soon as it receives both of the two messages. Once $c$ notices its wrong retransmission, it as initiator starts a revocation process, in which the inconsistent information is erased from $\mathcal{M}$. More specifically, $c$ sends a revocation message following the forward path of $a$ 's registration message. The revocation message stops at a node where $a$ 's registration message stopped propagating. All the nodes that receive this revocation message remove $a$ 's information from their local repositories. Such a revocation process can possibly lead to chain effect. That is, the registration message of an SP node previously incorrectly blocked will now continue to propagate until the blocking rule is satisfied again.

Before an SP leaves the network or turns to "unavailable" status, it starts a revocation process itself to remove its own information from $\mathcal{M}$; after it returns or becomes available again, it needs to re-register as a new SP. 


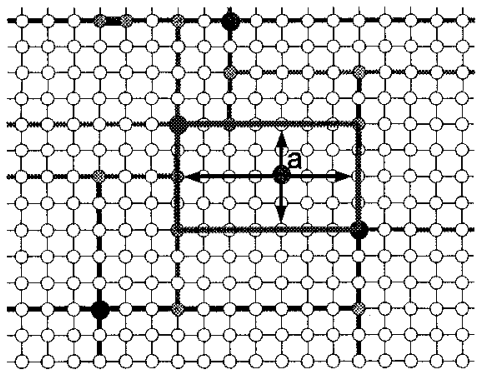

(a) In-cell case

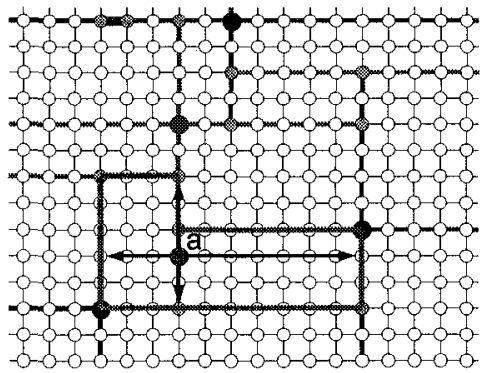

(b) On-edge case

Figure 5.2: Cross lookup

\section{Distance-sensitive service lookup}

For a SC node $a$, the objective of its service lookup is to identify the location of its target service provider $T(a)$ (see below for definition). According to the position of $a$, there are two possible lookup cases: in-cell case and on-edge case.

Definition 5.3 (Home Cell). The home cell HCell(a) of a SC $a$ is the mesh cell where $a$ is located in or the aggregation of the mesh cells which a is adjacent to.

Definition 5.4 (SPV). The Set of Providers in Vicinity (SPV) of a SC a is the set of SPs that distribute their information along the perimeter of $H C$ Cll $(a)$.

Definition 5.5 (Target Service Provider). The target service provider T(a) of a SC $a$ is the nearest $S P$ in the $S P V$ of $a$.

In the in-cell case, the SC node $a$ is located inside a cell of $\mathcal{M}$. When $a$ wants to find $T(a)$, it sends a search message along its residing grid row and column in four directions, as shown in Figure 5.2(a), where the home cell of $a$ is marked by thick gray lines and its search paths are highlighted by arrowed black lines. Such a search message stops its further transmission as soon as it hits a mesh edge (or the border of $G$ ), and then the node at which the message stops replies $a$ with the location of its recorded SP node closest to $a$ (resp., a failure notice). If there is no SP in the network, what $a$ will receive are all failure notice; otherwise, $a$ can find 


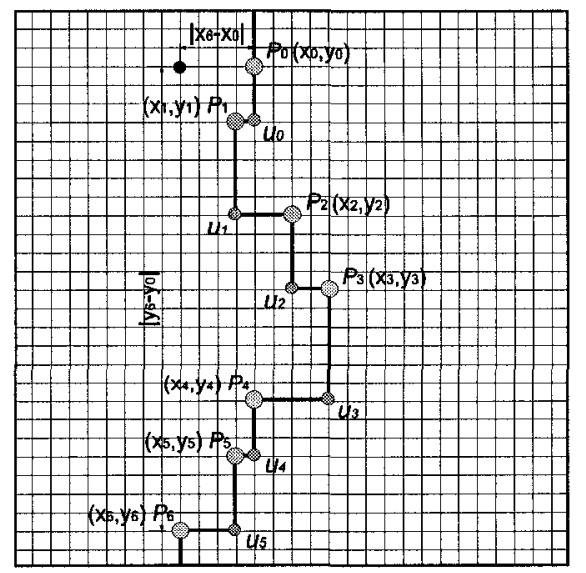

Figure 5.3: Blocking chain $p_{0} \stackrel{6}{\Leftarrow} p_{6}\left(p_{0} \stackrel{u_{0}}{\leftarrow} p_{1} \stackrel{u_{1}}{\leftarrow} \ldots \stackrel{p_{5}}{\leftarrow} p_{6}\right)$

the location of $T(a)$ simply by a local comparison among its received location data. Because the search paths of $a$ form a cross, this service lookup method is called cross lookup.

The cross lookup method can also be applied to the on-edge situation, namely, when the SC node $a$ is riding on an edge of $\mathcal{M}$. In this case, the search message that travels along a residing mesh edge of $a$ will stop at the farthest end of the mesh edge on the home cell perimeter, as illustrated by Figure 5.2(b). By this means, $a$ can reach all the composing mesh edges of its home cell and make a right decision.

\section{Analysis}

In this section, we shall explore the theoretical aspects of iMesh-A. As we will see, iMeshA has low message complexity and optimal per node storage load; however it does not always provide perfect nearby service selection guarantee (rare counterexample cases exist).

Definition 5.6 (Chain Blocking). For two SPS $a$ and $b(a \neq b), b$ is said "chain-blocking $a$ at length $k$ " if there is a blocking chain of length $k(k \geq 1)$ from $b$ to $a$, i.e., $a \stackrel{u_{0}}{\leftarrow} \ldots \stackrel{u_{k-1}}{\leftarrow} b$. We denote this chain blocking by $a \stackrel{k}{\models} b$ or $b \stackrel{k}{\Rightarrow}$ a. Figure 5.3 shows a blocking chain $p_{0} \stackrel{6}{\Leftarrow} p_{6}$ along $Y$ axis. 
Lemma 5.1. In a blocking chain $a \stackrel{k}{\Leftarrow} b$ along $Y$ (resp., $X)$ axis, the distance between $a$ and $b$ along $X$ (resp., $Y)$ axis is not longer than their distance along $Y(r e s p ., X)$ axis.

Proof. Suppose the blocking chain is along Y axis. Consider any two consecutive SP nodes $p_{i}$ and $p_{i-1}(1 \leq i \leq k)$ in the blocking chain. Denote their coordinates respectively by $\left(x_{i}, y_{i}\right)$ and $\left(x_{i-1}, y_{i-1}\right)$. We have $\left|x_{i}-x_{i-1}\right| \leq\left|y_{i}-y_{i-1}\right|$ because, otherwise, $p_{i}$ can not block $p_{i-1}$ in Y-direction. In this case, $\left|x_{k}-x_{0}\right|=\left|\sum_{i=1}^{k}\left(x_{i}-x_{i-1}\right)\right| \leq \sum_{i=1}^{k}\left|x_{i}-x_{i-1}\right| \leq \sum_{i=1}^{k}\left|y_{i}-y_{i-1}\right|=$ $\left|\sum_{i=1}^{k}\left(y_{i}-y_{i-1}\right)\right|=\left|y_{k}-y_{0}\right|$. Similarly, if the blocking chain is along $\mathrm{X}$ axis, we can prove $\left|y_{k}-y_{0}\right| \leq\left|x_{k}-x_{0}\right|$. Hence, the lemma holds.

Definition 5.7 (Extension). The extension $\eta(\mathcal{M})$ (or $\eta$ for brevity) of $\mathcal{M}$ is the length sum of the edges in $\mathcal{M}$.

Lemma 5.2. In a square $G, \eta \in O(\operatorname{Min}\{v \sqrt{n}, n\})$

Proof. For a complete mesh that is constructed without applying the block rule, its extension is just the product of $\sqrt{n}$ and the number $t$ of its constituting grid rows and columns. Clearly, the maximum value of $t$ is $2 v$, for example, in the case that there are no horizontally or vertically collinear SP nodes. Therefore, the extension of the complete mesh is bounded above $O(v \sqrt{n})$. Because $\mathcal{M}$ is the result of edge pruning of the complete mesh structure by the blocking rule, its extension is naturally bounded above $O(v \sqrt{n})$ as well. This upper bounder is actually achievable, for example, when SP nodes are all located on the same line along X-axis (or Yaxis). Note that, when $v>\sqrt{n}, v \sqrt{n}$ can be much larger than $n$ in order of magnitude for large $n$. Furthermore, since $\mathcal{M}$ is accommodated within $G$, its extension $\eta$ obviously never exceeds $2 n-2 \sqrt{n}=O(n)$, the total number of edges in $G$. Hence, $\eta \in O(\operatorname{Min}\{v \sqrt{n}, n\})$.

Lemma 5.3. In a square $G, \eta=\Omega(v+\sqrt{n})$.

Proof. In $\mathcal{M}$, every SP node has exactly four incidental edges, each shared by two SP nodes at most, and thus the number of mesh edges is not less than $2 v$. Under this circumstance, because each mesh edge has length at least $1, \eta$ is bounded below $O(v)$. Now, let us consider 


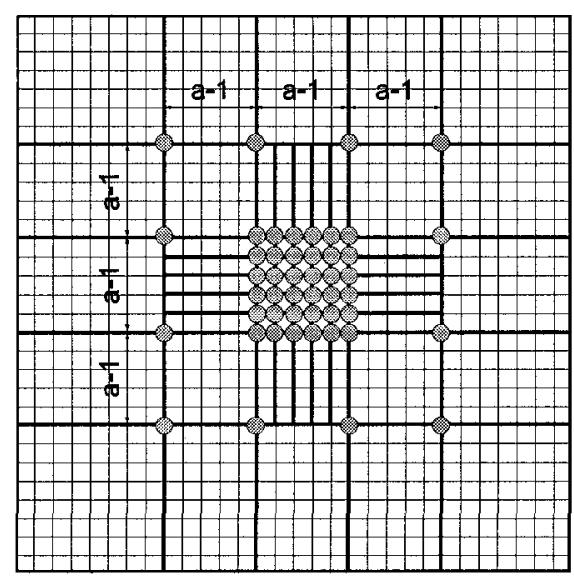

Figure 5.4: An information mesh of $\eta=O(v+\sqrt{n})$

a northmost SP node $p_{0}$. If $p_{0}$ is not blocked along Y-axis, its entire residing column will be included in $\mathcal{M}$; otherwise, there must exist a blocking chain spanning the entire network along Y-axis. In either case, $\eta$ is not less than $\sqrt{n}$. By the above analysis, $\eta=\Omega(v+\sqrt{n})$.

Note that, the lower bound indicated by Lemma 5.3 is achievable, for example, in the scenario shown in Figure 5.4. In this example, there are $v=a^{2}+12$ SP nodes: $a^{2}$ are densely packed in the middle of $G$, constituting an $a \times a$ inner grid; 12 are evenly placed around the inner grid at distance $a-1$, forming a big square that blocks the inner grid expanding. The length summation of the mesh edges is less than $6 v$ (in fact, it should be $6 v-6(a+12)$ ) inside the big square; on the outside, it is no more than $8 \sqrt{n}$ (in fact, it should be $8 \sqrt{n}-12(a-1)$ ). Thus in total is $\eta<8 \sqrt{n}+6 v=O(v+\sqrt{n})$.

Theorem 5.1. In a square $G$, the message complexity of information mesh construction is $O(\psi(G))$, where $v+\sqrt{n} \leq \psi(G) \leq \operatorname{Min}\{v \sqrt{n}, n\}$.

Proof. If $G$ is a synchronous environment, the paths that SP nodes' registration messages travel are exactly the edges of $\mathcal{M}$. In this case, due to the blocking rule, a constant number (1 or 2 ) of registration messages are transmitted on each communication link in these mesh edges. Specifically, there are two registration messages transmitted on the middle link of two collinear SP nodes separated by an odd number of hops (as the case with $c$ and $e$ in Figure 
5.1(b)), and one registration messages over all the other links. Hence, the theorem follows immediately from Lemma 5.2 and 5.3. If, otherwise, $G$ is an asynchronous environment, because some registration messages may be incorrectly transmitted on the links in $G-\mathcal{M}$, and revocation messages are used for consistency maintenance, the message complexity can not be lower than in an synchronous scenario. On the other hand, because SP nodes still block messages effectively, there are at most 4 messages, 2 in each direction, transmitted on each link in the complete mesh structure, and as a consequence the message complexity can not be worse than $O(\operatorname{Min}\{v \sqrt{n}, n\})$. Hence, the theorem holds.

Theorem 5.2. In a square $G$, the message complexity of cross lookup is $O(\sqrt{n})$.

Proof. A cross lookup process of a SC node is restricted within a search cell, i.e., the home cell of the SC node. In worst case, for example, when SP nodes are all located on the same network border, a search cell spans the entire network, and a SC node in the search cell will inquire all the way along its residing grid row and/or column, generating $O(\sqrt{n})$ search messages. This proves the theorem.

Theorem 5.3. iMesh generates constant storage load on each network node.

Proof. At any time, each of the nodes that constitute $\mathcal{M}$ records at most one SP node's information from each of the four directions, i.e., the north, the south, the west and the east, due to the blocking rule. The nodes which are not part of $\mathcal{M}$ do not store any data at all. Hence, the theorem holds.

Definition 5.8 (Target over Closest Ratio). The target over closest ratio TCR(a) of a SC $a$ is defined as $\operatorname{TCR}(a)=\frac{|a T(a)|}{|a C(a)|}$, where $C(a)$ is an SP closest to a.

TCR measures the distance sensitivity of iMesh. Ideally, $\operatorname{TCR}(a)$ is equal to 1 , meaning closest service selection. This happens when the residing grid row and/or column of $C(a)$ is part of the perimeter of $H C$ ell $(a)$. However, due to randomized distribution of SPs, it may not always be the case. To study the distance sensitivity of iMesh-A, all the possible violation 


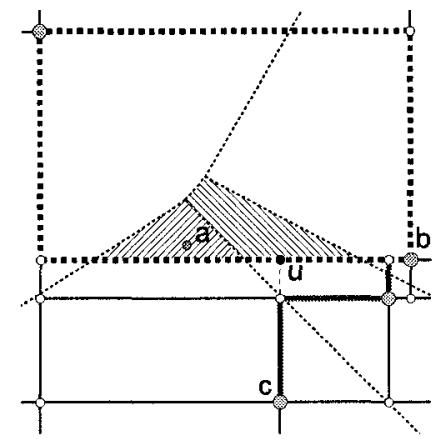

(a) Barrage Case

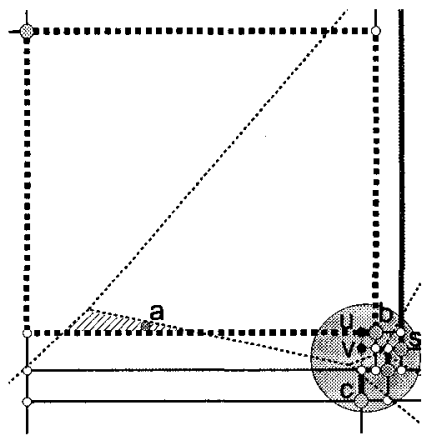

(b) Clean-Pass Case

Figure 5.5: Example situations of $1<T C R(a) \leq 2$

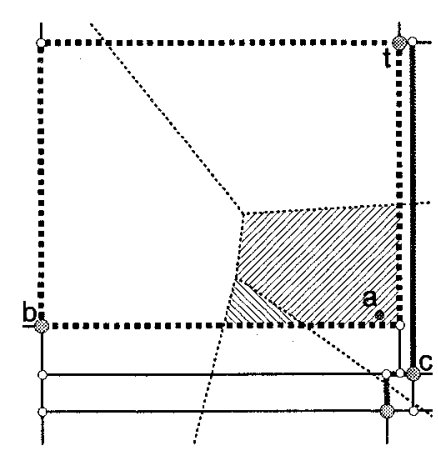

(a) Dirty-Pass Case

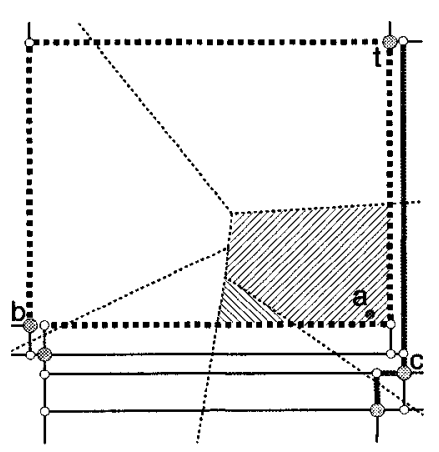

(b) Isolation Case

Figure 5.6: Example situations of $T C R(a)>2$

situations where TCR $>1$ need to be identified. By an exhaustive search, it is observed that all violations are the variants of the following four basic cases:

(1) Barrage case: $C(a)$ is chain-blocked by an SP in a's SPV, before its blocking chain passes around $H C e l l(a)$;

(2) Clean-Pass case: the blocking chain of $C(a)$ passes around $H C e l l(a)$ at the corner where an SP is located;

(3) Dirty-Pass case: the blocking chain of $C(a)$ passes around $H C e l l(a)$ at the corner where no SP exists, and a composing mesh edge of $H C$ ell $(a)$ intersects the residing mesh edge of $C(a)$; 


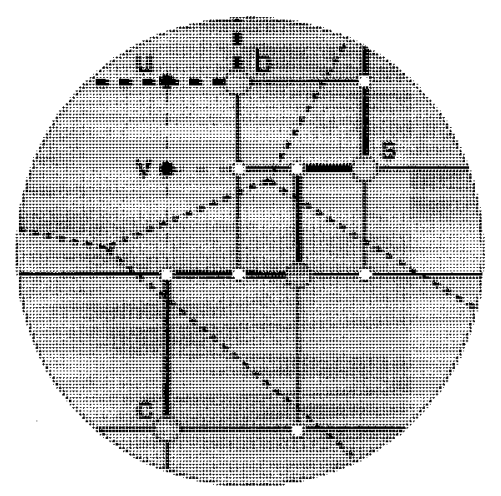

Figure 5.7: An amplified version of the circled part in Figure 5.5(b)

(4) Isolation case: the blocking chain of $C(a)$ passes around $H C e l l(a)$ at the corner where no SP exists, and no composing mesh edge of $H C e l l(a)$ intersects the residing mesh edge of $C(a)$.

Figures 5.5 and 5.6, where irrelevant SC nodes are hidden and SP nodes are represented by solid big dots, illustrate the above four basic violation cases. In the two figures, the home cell $H C$ ell $(a)$ of SC node $a$ is emphasized by broken thick lines, and the blocking chain of $c=C(a)$ is highlighted by complete thick lines; broken thin lines indicate the Voronoi diagram created using SP nodes, and shadowed areas are the places where TCR is greater than 1 .

Lemma 5.4. In Barrage case, $T C R(a) \leq 2$.

Proof. Let $b$ be the SP node in $a$ 's SPV that chain-blocks $c$ (i.e., $C(a)$ ). We have $|a T(a)| \leq|a b|$. Without loss of generality, assume that the chain of blocking happens along Y-axis, as shown in Figure 5.5(a). By Lemma 5.1, $|b u| \leq|c u|$. Observe that angle $\angle c u a$ can not be acute in any case. Thus $c a$ is the longest side in triangle $\Delta c u a$. Namely, $|c u|<|c a|$ and $|u a|<|c a|$. Then $|a b| \leq|b u|+|u a| \leq|c u|+|u a|<|c a|+|c a|=2|c a|$. Because $|a T(a)| \leq|a b|$, we have $|a T(a)| \leq 2|c a|$, which completes the proof.

Lemma 5.5. In Clear-Pass case, $T C R(a) \leq 2$.

Proof. Without loss of generality, assume that the blocking chain of $c$ (i.e., $C(a)$ ) is toward $H C e l l(a)$ along Y-axis, as shown in Figure 5.5(b). Examine the amplified version in Figure 
5.7. By Lemma 5.1, $|s v| \leq|v c|$. Unambiguously, $|b u| \leq|s v| \leq|c v| \leq|c u|$. From this point, the lemma then follows from the same proof as Lemma 5.4.

Lemma 5.6. In both Dirty-Pass case and Isolation case, TCR(a) may be larger than 2 but can not be larger than $\frac{d(G)}{|a C(a)|}$, where $d(G)$ is spatial the diameter of $G$.

Proof. Let us examine the scenarios given in Figure 5.6, where $t=T(a)$ and the blocking chain of $c$ (i.e., $C(a)$ ) is along Y-axis. By observation, $|a t|$ is already greater than $2|a c|$, namely, $\operatorname{TCR}(a)>2$, and there is no restriction on the distance from $t$ to the residing grid row of c. If we move $b$ (together with $d$ in Figure 5.6(b)) and $t$ far apart from $a$ while maintaining their blocking relation, then $|a t|$ could be way larger than $2|a c|$. On the other hand, because no pair of nodes have their separation larger than $d(G)$, we have $|a t| \leq d(G)$ and consequently $\operatorname{TCR}(a)=\frac{|a t|}{|a c|} \leq \frac{d(G)}{|a c|}$.

In [77], the authors claimed that the blocking rule allows a node to discover a service that is at a distance no larger than $\sqrt{2}$ times the distance from the closest. Lemma 5.6 indicates that their claim is false.

\subsubsection{Complete iMesh}

A distance-sensitive service discovery algorithm is expected to guarantee nearby service selection, that is that $T C R(a) \leq 2$ for any SC node $a$. By Lemma 5.6, iMesh-A may violate this expectation in Dirty-pass case and Isolation case. In this section, we will present the complete version of iMesh, $i$ Mesh- $B$, which achieves major improvement on distance sensitivity over, but has the same complexity as, iMesh-A.

Define the territory of an arbitrary SP node $c$ as the area in which $c$ can be discovered by the local SCs through the cross lookup method (refer to Section 5.3.1 for cross lookup). The larger the territory of $c$, the higher its probability of being discovered, and thus the better the distance sensitivity of iMesh. However, in iMesh-A, the size of an SP's territory is strictly restricted by the blocking rule for message saving purpose. Figure 5.8 redraws the Dirty-Pass 


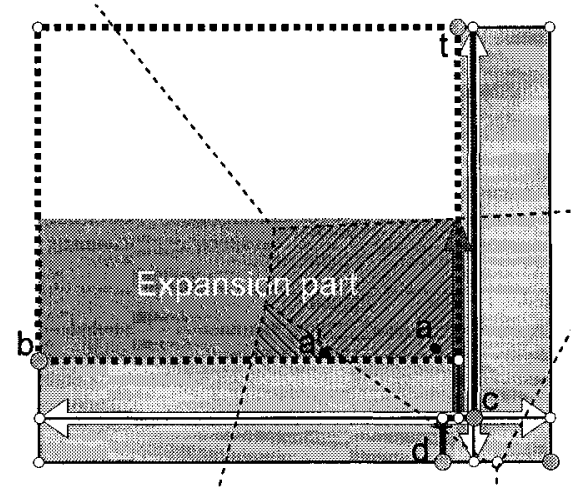

Figure 5.8: The effect of the extension rule

situation given in Figure 5.6(a). In this figure, the territory of SP node $c$ is represented by the light gray area, which is actually the aggregation of the mesh cells adjacent by the registration paths (marked by arrowed hollow lines) of $c$.

In order to improve the distance sensitivity of iMesh, territory expansion is necessary. In iMesh-B, the information mesh is built not only according to the blocking rule but also using an extension rule. The new extension rule enables SPs to expand their territories in the case of orthogonal blocking. The formal definition of the extension rule is given below:

Rule 5.2 (Extension Rule). A node $u$ at which an SP node a orthogonally blocks another $S P$ node $b$ sends the information of $a$ to $b$ along the backward path from which it receives $b$ 's information. The information of a does not travel all the way to $b$ but stops at the point where the path intersects the bisector between $a$ and $b$.

In Figure 5.8, the transmission paths of the extension messages of SP node $c$ is highlighted by arrowed solid lines, and the dark gray area is the expansion part of the territory of $c$. By observation, $c$ 's territory expands into the home cell $H C$ ell $(a)$ of SC node $a$, and $a$ becomes able to discover $c$ as a result. Consider another SC node $a^{\prime}$ that shares the same home cell with $a$. The closest SP node $C\left(a^{\prime}\right)$ to $a^{\prime}$ is $d$ in the blocking chain of $c$. In iMesh-A, $T C R\left(a^{\prime}\right)$ could be way greater than 2 (if $H C$ ell (a) is very large) according to Lemma 5.6. On the contrary, in iMesh-B, we have $T\left(a^{\prime}\right)=c$ and then $T C R\left(a^{\prime}\right) \leq 2$ following a similar proof as Lemma 5.4. 
By the above examples, the extension rule eliminates the Dirty-Pass case, and thus the negative Lemma 5.6 only holds partially for iMesh-B. By definition, the extension rule does not either change the structure of, or remove any data from, the information mesh. Therefore, Lemma 5.2, 5.3, 5.4 and 5.5 and Theorem 5.2 still hold for iMesh-B. In addition, it is not difficult to verify that Theorem 5.1 and 5.3 are also applicable to iMesh-B. In summary, the extension rule enables iMesh-B to achieve improved overall distance sensitivity over iMesh-A at very low cost. Its effect and cost will be seen clearly later, through simulation in Section 5.5 .

\subsection{Implementation details}

In an arbitrary sensor network, there is no grid structure that we can make use of for information mesh construction and cross service lookup. Under this circumstance, we accomplish our goal by using routing protocol GFG $[15,27]$, which is known for its guaranteed packet delivery and has been used to support quorum formation in Quorum [48, 72, 74].

\subsubsection{Information mesh construction}

An arbitrary SP generates four registration messages carrying its location information respectively for the four directions, i.e., the north, the south, the west, and the east. Then it sends them to the corresponding directional foremost neighbors, namely, the northbound message to the northmost neighbor, and the southbound message to the southmost neighbor, and so on. These registration messages are retransmitted by receiver nodes following protocol GFG. More specifically, upon receiving a registration message, a node retrieves the embedded SP node information from the message, stores it in local storage, records the message's designated transmission direction and then greedily forwards the message to its foremost neighbor in the same direction. When a registration message reaches a void area, it is switched to the face routing mode and then passed around the void area in the clockwise (counterclockwise) direction by the left (resp., right) hand rule. Greedy forwarding resumes whenever possible. 


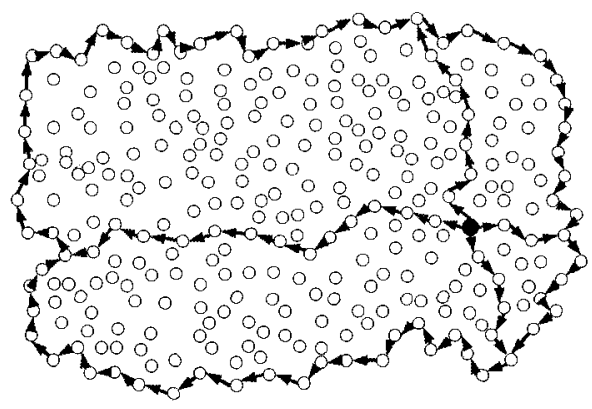

(a) One SP node

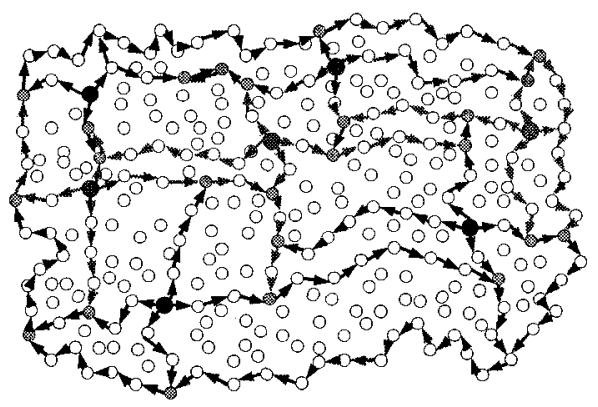

(b) Seven SP nodes

Figure 5.9: Information mesh construction in an arbitrary sensor network

If the source is the only SP in the network, due to the absence of the network's boundary information and the nature of GFG, a registration message will finally stop at the globally foremost node in its transmission direction, and its transmission path will include the entire network boundary, as shown in Figure 5.9(a) where the registration paths (i.e., the transmission paths of the registration messages) of the only SP is highlighted by arrowed lines. In the case that there is more than one SP in the network, SPs' registration paths intersect one and another inside the network and/or overlap on the network boundary. For two intersecting registration paths, they will be either in a node-sharing situation or in a link-crossing situation. In the former case, the two paths intersect at a common node, while in the latter case, they have a pair of crossover links. As explained in Appendix A.1, a link-crossing situation can be locally (without extra message transmission) transformed to a node-sharing situation in a sensor network modeled as unit disk graph.

By above analysis, for any two different SPs, their registration paths are guaranteed to have some nodes in common. Then these common nodes apply the blocking rule as in the context of grid sensor networks. Finally, an information mesh structure is established as a result. Figure 5.9(b) shows an information mesh created by seven SP nodes in an arbitrary sensor network. In this figure, SP nodes and their registration paths are differentiated by different colors, and gray dots represent the nodes where the blocking rule applies. 


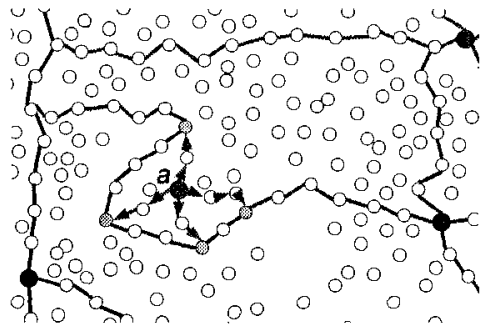

(a) Curly edge

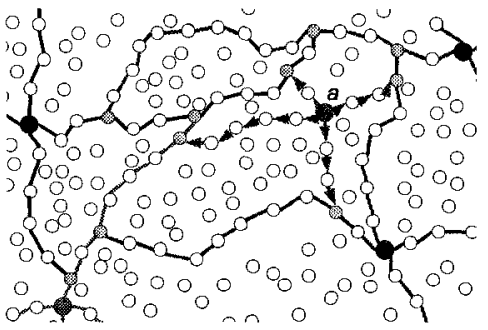

(b) Irregular shape

Figure 5.10: Cross lookup in an arbitrary sensor network

\subsubsection{Distance-sensitive service lookup}

The implementation of cross lookup is simple. A SC node $a$ sends a query message to its directional foremost neighbors. Each of these messages is retransmitted through protocol GFG and stops at the first receiver node that resides on the information mesh. Then this receiver node sends $a$ a positive reply containing its locally stored SP information. However, if there does not exist any SP in the network, which is possible when all the SP nodes become unavailable, such a query message will reach a boundary node $b$ and then traverse the entire network boundary starting from there, by the property of GFG. In this case, once the query message gets back to $b$ along the network boundary, $b$ sends $a$ a negative reply, indicating the failure of service lookup. If all received replies are positive, $a$ can easily determine its target service provider $T(a)$; if at least one of them is negative, it knows its service lookup fails.

If no void area appears in the network topology, the greedy forwarding part of GFG will never fail. As a result, every cell in the information mesh has a rectangular shape, and thus the cross lookup method always works. However, in the presence of void areas, messages are routed along the perimeters of the void areas, causing zigzag message transmissions and thus possible cross lookup failures. Figure 5.10, where arrowed gray lines indicate search paths, shows two examples. In the scenario demonstrated by Figure 5.10(a), the search messages of SC node $a$ all hit the same curly edge of its home cell; in the scenario illustrated by Figure $5.10(\mathrm{~b})$, the home cell of $a$ is composed of five edges, causing that no search message reaches the northmost edge. Apparently, $a$ fails to find its true target service provider in the two cases. 


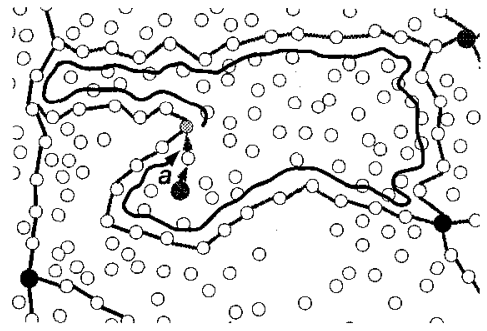

(a) Curly edge

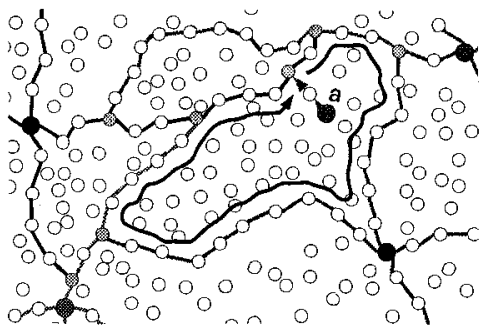

(b) Irregular shape

Figure 5.11: Perimeter lookup in an arbitrary sensor network

To ensure successful nearby service lookup in such undesirable situations, an alternate perimeter lookup method can be used. By this method, any SC $a$ sends a search message to an arbitrarily selected direction through protocol GFG. This search message will hit $a$ 's home cell perimeter at certain node, called entry node, which then retransmits the message along the cell perimeter, e.g., in the clockwise direction. The search message picks up the information of the closest SP that it have seen during its perimeter traversal. After it travels all the way along the cell perimeter back to the entry node, it has found the target service provider $T(a)$ of $a$. Therefore, upon receiving the search message back, the entry node returns the message to $a$ as a reply. This perimeter lookup method is illustrated in Figure 5.11 where light blue dots denote the entry nodes that start perimeter traversal. A special case is that a SC is riding on the information mesh. Under this circumstance, the SC performs the perimeter lookup in its every home cell. But since it is already on its home cell perimeter in this case, it can start perimeter traversal directly.

\subsubsection{Tolerating node failures}

Node failure may lead to loss of SP information. Similar to the fault-tolerance approach employed in Quorum [48, 72,74], iMesh uses thick registration paths to increase information redundancy and consequently its fault-tolerance capability. Specifically, during the information mesh construction process, SP nodes' registration messages are transmitted along paths of certain thickness. For thickness 1 , all the nodes that overhear a registration message store 
the embedded SP location information; for thickness $k$, these overhearing nodes are required to broadcast the registration message within their $(k-1)$-hop neighborhood.

Another impact from node failure is loss of control messages for service lookup. To tolerate such message loss, iMesh uses a simple yet effective fault-tolerance approach, transmission retrial. That is, during a service look up process, if the SC node does not get any reply to its lookup message, it backs off for a while and then retries.

It is also possible that SP nodes fail without notification. To handle SP failures, iMesh requires that the neighbors of each SP monitor the SP node's liveness, e.g., by listening to a periodic beacon message. Once they find the SP node fails, they immediately start a revocation process (refer to Section 5.3.1) to removes the SP's information from the information mesh.

\subsubsection{Keeping storage load constant}

According to the way of information mesh construction, the storage load of a node is subject to the number of its incidental mesh edges. In an arbitrary network scenario, the number of nodal incidental mesh edges is bounded above node degree, which is an inconstant value and can be equal to $n-1$ in the worst case. To keep storage load constant, an node where the blocking rule applies does not store the location information that it blocks but adds a mark (nearly at no extra storage cost) to the neighbor from which it receives the blocked information, such that it can later find the blocked information without actually storing it.

\subsection{Performance Evaluation}

As summarized in Section 1.2.1 and 2.3.2, existing service discovery algorithms and adoptable techniques usually rely on global computation and therefore generate large message overhead, and they may in addition impose inconstant storage load on network nodes and/or induce bottleneck problem in the network. Our proposed protocol iMesh however has obvious advantages in all these aspects. It aims to yield optimal (constant) per node storage load and 
avoid long service registration/lookup paths while providing satisfactory distance-sensitivity.

In the case that no comparable work actually exists, we choose to evaluate iMesh in comparison with Quorum $[48,72,74]$, through an extensive set of simulation. As we will see in the following, iMesh has considerably low message overhead when compared with Quorum, and iMesh-B guarantees closest service selection with high probability, larger than $97 \%$, and nearby service selection with very high probability, larger than $99 \%$, significantly impoving the distance sensitivity of iMesh-A at negligible communication cost.

\subsubsection{Evaluation metrics}

We study the message overhead of iMesh in comparison with Quorum's using the following metrics:

- Total Number of Construction Messages (TNCM): the total number of messages transmitted in the network for information mesh construction;

- Number of Construction Messages per SP (NCMSP): the average number of messages generated by an SP for the purpose of information mesh construction;

- Number of Search Messages per SC (NSMSC): the average number of service lookup messages generated by an arbitrary SC (reply messages are not counted);

As Quorum guarantees closest service selection (i.e., TCR $=1$ ), the following evaluation metrics are for iMesh only:

- Average TCR and Peak TCR: the average TCR and the peak TCR of all the possible SCs in the network.

- PTCR1, PTCR2, and PTCR3: the probability of TCR = 1, $1<\mathrm{TCR} \leq 2$, and TCR $>2$.

\subsubsection{Simulation setup}

We simulated iMesh-A, iMesh-B and the Quorum within a custom network simulator. Our simulation was carried out over a large-scale WSN, which contains $1,000,2,000, \ldots, 10,000$ 


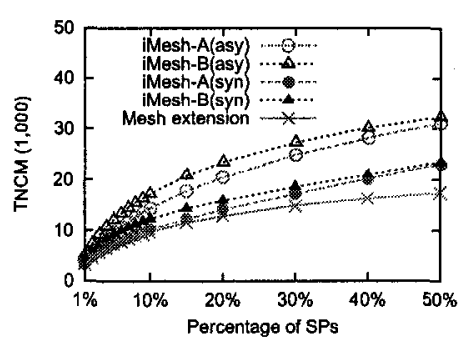

(a) TNCM

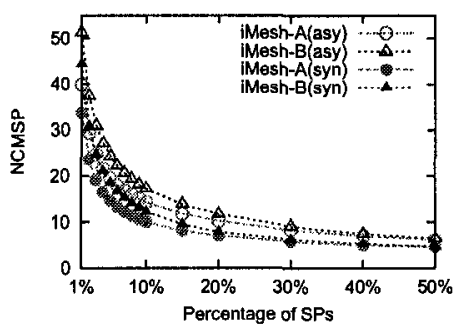

(b) NCMSP

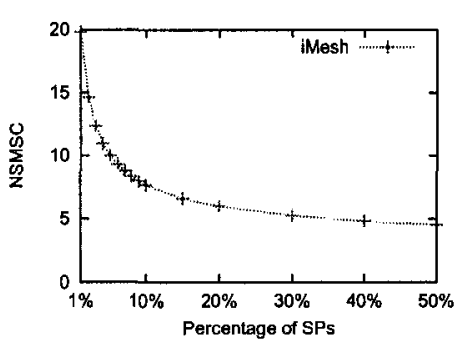

(c) NSMSC

Figure 5.12: Message overhead in a WSN of 10,000 nodes

nodes and fully covers a square sensory field with average node density $8-9$. The scenarios with larger node densities are also tested; but the results are even better (because less nodes are involved in message transmissions) and therefore off our interest.

We run two sets of experiments. In the first set, the network is set to be a synchronous environment with simultaneous execution and unified link delay; in the second set, the network is configured to be an asynchronous environment where SP nodes start the protocol maximally 30 simulated time units off each other, and each communication link has transmission delay of 10 simulated time units at most. We choose the settings with the percentage of SPs (PSP) in the network varying from $0.1 \%$ to $50 \%$. For each setting, we executed iMesh-A, iMesh-B and Quorum over 100 randomly generated network scenarios to get average results.

\subsubsection{Experimental results}

We shall now elaborate on our experimental results, whose confidence interval is within $15 \%$. We will closely examine the message overhead and the distance sensitivity of iMesh in a network of size 10,000. We will show when iMesh outperforms Quorum [74] in message overhead based on the results obtained in the networks of size $1,000,2,000, \ldots, 10,000$.

\section{Message overhead}

We first study the communication cost of the two versions of iMesh in a synchronous environment and in an asynchronous environment with 10,000 nodes. Later, in Section 5.5.3, 
we will further analyze the message overhead of iMesh in other sized networks, in comparison with protocol Quorum [74].

Figure 5.12(a) show the TNCM of iMesh in relation with PSP. For reference, mesh extension (Definition 5.7) is also drawn in the figure. As PSP grows, the information mesh has a more and more complex structure and is therefore expected to exhibit an increasing extension and a growing construction message overhead. The expectation is confirmed by the ascending trend of the curves in the figure. The small gap between the TNCM curves for iMesh-A and iMesh-B in either environment indicates that the overhead of the extension rule (Rule 5.2) is minor. And, from the figure we can also see that TNCM will never exceed some constant times mesh extension. This observation verifies Theorem 5.1.

Examine again Figure 5.12(a) and pay attention to the difference of TNCM in the two environments. It is observed that TNCM is always higher in the asynchronous environment than in the synchronous environment. This is due to the extra messages used for eliminating the information inconsistency caused by asynchrony. Further, as PSP grows in either environment, TNCM curves deviate more and more from the curve of mesh extension, and the TNCM of iMesh-B approaches to the that of iMesh-A closer and closer. It is because, when there are more SP nodes, the situation that two collinear SP nodes are an odd number of hops away happens more often, causing more overlapping registration messages on mesh edges, and the mesh cell has smaller size, leading to the reduction of the travel distance of extension messages.

Figure 5.12(b) displays the NCMSP of iMesh as a function of PSP. From the figure, we can see that NCMSP drops and approaches to 4 as PSP goes up. It is because, when SP density increases, an SP node's registration message travels a decreased hop-distance (on average) in each direction before being blocked, and the travel distance can be as low as 1-hop, resulting in merely 4 registration messages in the extreme case. As shown in the figure, each SP node uses slightly more construction messages in the asynchronous environment than in the synchronous environment due to the cost of information consistency maintenance; iMesh-B 


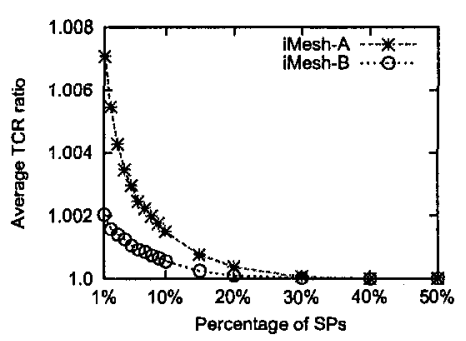

(a) Average TCR

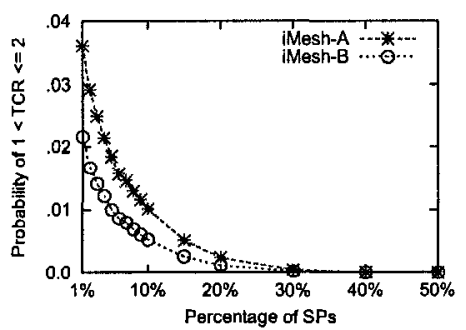

(d) PTCR2

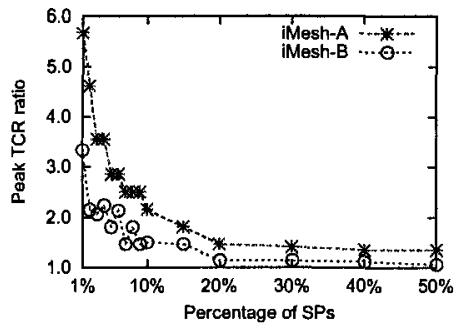

(b) Peak TCR

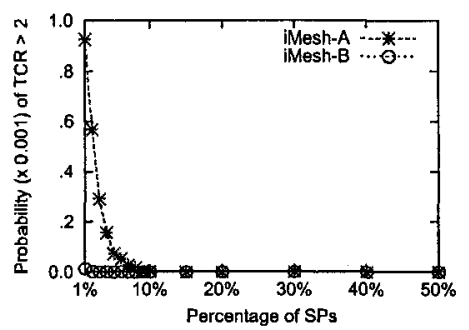

(e) $\operatorname{PTCR} 3\left(\times 10^{-3}\right)$

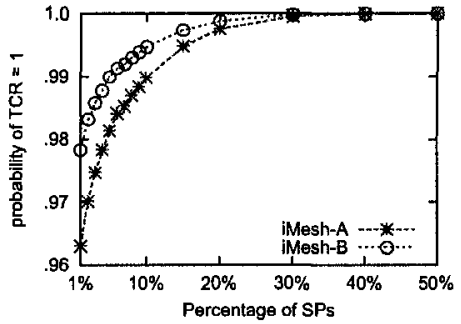

(c) PTCR1

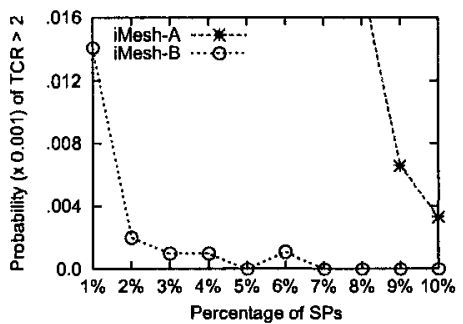

(f) PTCR3 with $1 \% \leq$ PSP $\leq 10 \%$

Figure 5.13: Distance sensitivity in a network of 10,000 nodes

generates slightly larger NCMSP than iMesh-A in both environments, which again implies the negligible message cost of the extension rule.

Figure 5.12(c) depicts the NSMSC of iMesh, which is irrelevant to synchrony and to the application of the extension rule, as a result of PSP. It is observed that NSMSC drops and approaches to 4 as PSP climbs. It is because, when SP density increases, a SC node's search message travels a decreased hop-distance (on average) in each direction before finding an SP, and the travel distance can be as low as 1-hop, resulting in merely 4 search messages in the extreme case.

To sum up, the results given in Figure 5.12 clearly indicate that protocol iMesh (whether the A version or the B version) use a considerably small, compared with network size, number of messages for service registration and service lookup. At a detailed level, iMesh-B generates slightly larger message overhead than iMesh-A; but the difference is actually negligible. 


\section{Distance sensitivity}

We will now study the distance sensitivity of iMesh, which is regardless of the (synchronous or asynchronous) nature of the execution environment. In our simulation, iMesh exhibits consistent distance sensitivity in different sized networks. Below we will present the results from a network of 10,000 nodes only. The results from a network of size 1,000 can be found in Appendix A.2, while those obtained from other sized networks are omitted.

Figures 5.13(a) and 5.13(b) respectively show the average TCR and the peak TCR in relation with PSP. From Figure 5.13(a) we can see that the average TCR is nearly equal to 1 in all the PSP cases. This is because of the low probability of $T C R>1$. In both of the two figures, the curves decline and approach to 1 closer and closer as PSP increases. This phenomenon is due to the decreasing probability of TCR $>1$. According to the two figures, iMesh-B always has better distance sensitivity than iMesh-A. It is because the extension rule (Rule 5.2) effectively eliminates the Dirty-pass case (refer to Section 5.3.1 for definition).

Figures 5.13(c) - 5.13(e) depict PTCR1, PTCR2 and PTCR3 as a function of PSP. By Figure 5.13(c), both iMesh-A and iMesh-B provides closest service selection with high probability, respectively larger than 96\% and 97\%. By Figure 5.13(d) and 5.13(e), both PTCR2 and PTCR3 quickly drop down nearly to 0 as soon as the density of service providers increases to $10 \%$. The three figures together indicate that iMesh guarantees nearby service selection with very high probability, larger than 99\%, in all PSP cases, and they also confirm our analysis about TCR value in previous paragraph.

Figures 5.13(c) - 5.13(e) also imply that iMesh-B always has better distance sensitivity than iMesh-A. It is because iMesh-B eliminates the Dirty-Pass case by the extension rule. Examine the part for PSP in range 1\% - 10\% in Figure 5.13(e) (an amplified version of this part is given in Figure 5.13(f)). The PTRC3 of iMesh-A and iMesh-B are both extremely low, i.e., smaller than $O\left(10^{-3}\right)$. In particular, due to the application of the extension rule, iMesh-B's PTCR3 is significantly lower, in terms of oder of magnitude, than that of iMesh-A.

The experimental results shown in Figure 5.13 indicate that iMesh (whether version $\mathbf{A}$ or 


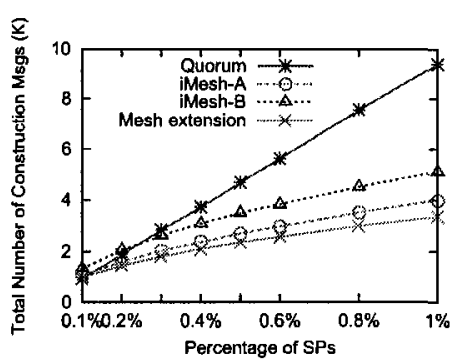

(a) TNCM

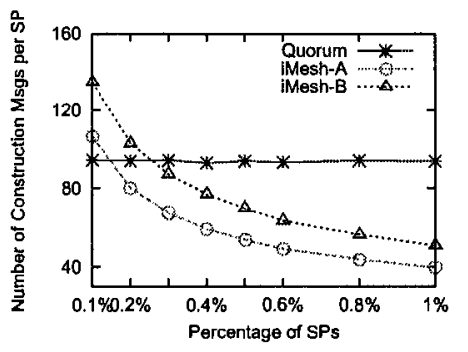

(b) NCMSP

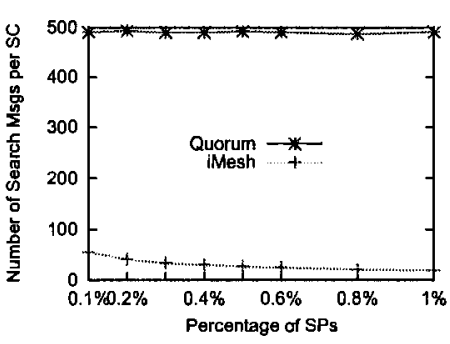

(c) NSMSC

Figure 5.14: iMesh v.s. Quorum in a WSN of 10,000 nodes

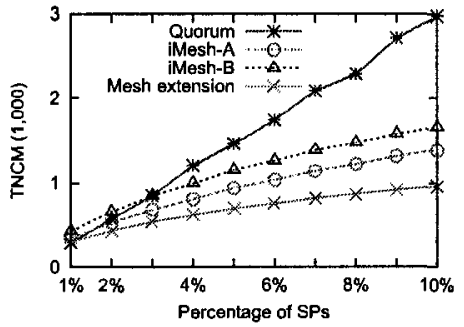

(a) TNCM

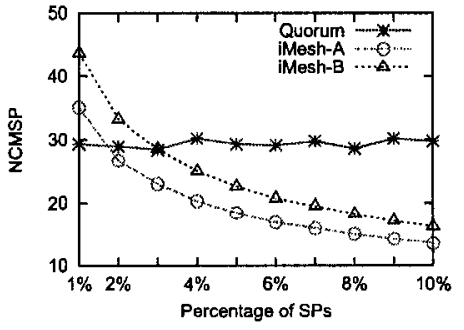

(b) NCMSP

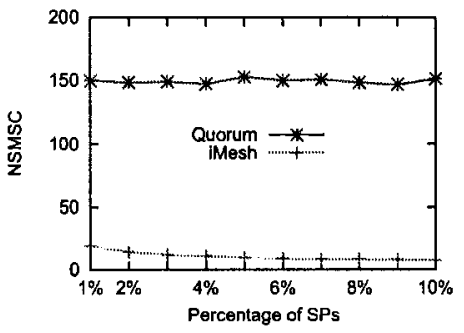

(c) NSMSC

Figure 5.15: iMesh v.s. Quorum in a WSN of 1,000 nodes

version B) has satisfactory distance sensitivity. Compared with iMesh-A, iMesh-B performs much better in both closest service selection aspect and nearby service selection aspect and has lower probability of undesired distance service selection. According to Section 5.5.3, iMesh-B in fact achieves these advantages over iMesh-A at the negligible cost of messages.

\section{iMesh v.s. Quorum}

As Quorum is irrelevant to network synchrony and guarantees closest service selection, we focus only on the difference between iMesh and Quorum in message overhead (measured by TNCM, NCMSP and NSMSC) in asynchronous environments. Let us first examine Figure 5.14 and 5.15, which show iMesh v.s. Quorum with varying PSP respectively in a WSN of size 10,000 and a WSN of size 1,000 .

Figure 5.14(a) and 5.15(a) depict TNCM as a function of PSP. It is observed that, as PSP increases, TNCM climbs quickly in Quorum but at a very slow speed in iMesh, almost starting 
from the same point. It is because, an SP's registration message always propagate across the entire network in Quorum; but, as discussed in Section 5.5.3, it travels a shorter and shorter distance due to message blocking in iMesh when PSP ascends.

Figure 5.14(b) and 5.15(b) exhibit NCMSP as a result of PSP. We can find that the curves corresponding to Quorum is nearly a horizontal line. It is because in Quorum an SP's registration message has to travel across the entire network, whose width is constant. On the contrary, iMesh has very low NCMSP due to message blocking, and the larger PSP, the more often message blocking happens, and therefor the lower NCMSP.

Figure 5.14(c) and 5.15(c) display NSMSC in relation with PSP. It is seen that that Quorum generates almost constant but dramatically larger NSMSC, regardless of PSP, when compared with iMesh. This phenomenon is reasonable because a SC in Quorum has to search across the entire network and along the whole outer boundary for a closest SP; while in iMesh, a SC does not query along the outer boundary of the network, and its service lookup operation is restricted within a search cell, whose size generally decreases as PSP increases.

The similarity in the trend of the curves in Figure 5.14 and 5.15 undoubtedly indicates the performance consistency of iMesh in different-sized networks. Let us now turn our attention to their difference, which is actually more of our interest.

In Figure 5.14, Quorum surpasses iMesh in TNCM and NCMSP (i.e., message overhead for service registration) for very small PSP ( $<0.3 \%$ in iMesh-A, $<0.2 \%$ in iMesh-B). The reason is quite obvious: cross registration paths used in iMesh lead to more messages in total when message blocking rarely happens. Similar crossover is also observed in Figure 5.15, but in a different PSP range ( $<2 \%$ in Mesh- $\mathrm{A}, \approx 3 \%$ in iMesh-B). However, this difference is important in that it provides us a guide about when iMesh can replace Quorum at best.

In order to ease our study on crossover point of iMesh and GRG in service registration overhead, we define Proportional Difference of Construction Cost (PDOCC) as follows: 


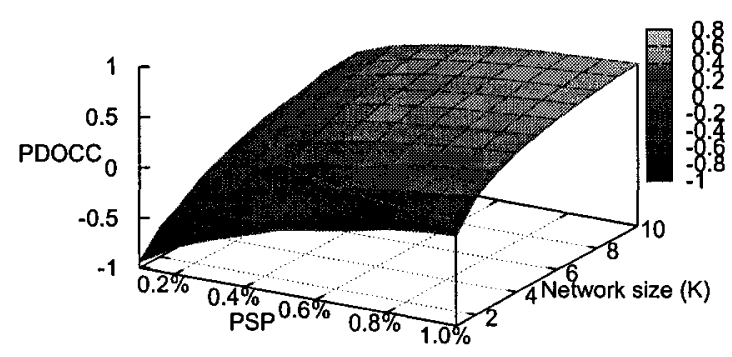

(a) iMesh-A

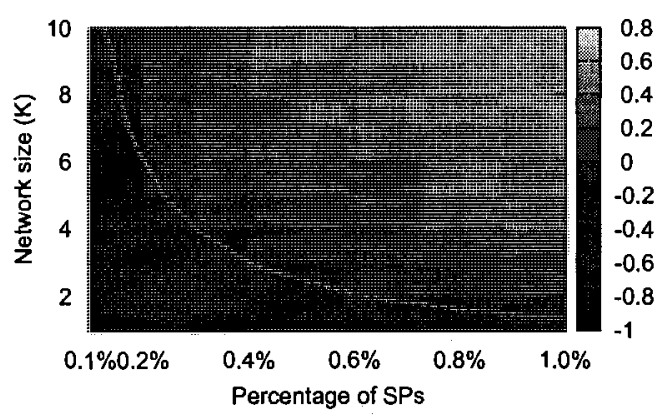

(c) iMesh-A

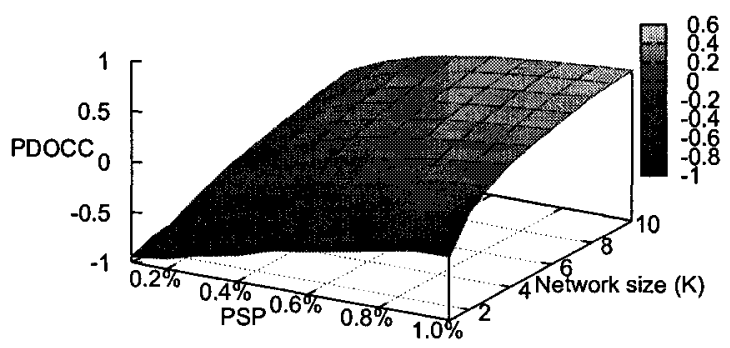

(b) iMesh-B

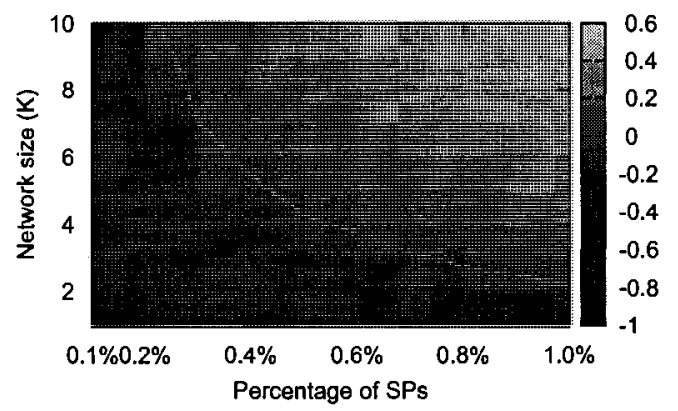

(d) iMesh-B

Figure 5.16: Proportional Difference of Construction Cost (PDOCC) of iMesh and Quorum

$$
\begin{aligned}
\text { PDOCC } & =\frac{\text { TNCM(Quorum)-TNCM(iMesh) }}{\text { TNCM(Quorum) }} \\
& =\frac{\text { NCMSP(Quorum)-NCMSP(iMesh) }}{\text { NCMSP(Quorum) }}
\end{aligned}
$$

By definition, the larger PDOCC is, the more advantageous iMesh is over Quorum; if PDOCC is negative, then iMesh is not as efficient as Quorum. In this case, a crossover point of $\mathrm{iMesh}$ and GRG will be a point where PDOCC $=0$. Figures 5.14 and 5.15 suggest that PDOCC starts to exhibit a positive value with a delayed PSP threshold, in order words, iMesh gradually becomes less and less advantageous than Quorum, as the network size descends, and that the degradation would be very slow compared with the decreasing speed of the network 
size. It is indeed true, as further confirmed by Figure 5.16 .

In Figure 5.16(a) and 5.16(b), the $\mathrm{X}, \mathrm{Y}$ and $\mathrm{Z}$ axises represent PSP, network size and PDOCC, respectively. The curves on the surfaces in the two 3D figures are plotted on the XY plane in Figure 5.16(c) and 5.16(d), respectively; they are composed of the crossover points of iMesh and Quorum and indicate the boundary beyond which a X-Y combination would yield a positive PDOCC of iMesh. For example, in a network of 6,000 nodes, iMesh-A produces positive PDOCC when PSP $>0.2 \%$, and iMesh-B does not until PSP $>0.3 \%$. By Figure 5.16 , in order for iMesh-A (iMesh-B) to generate positive PDOCC before PSP exceeds $1 \%$, the network should have a size larger than 1, 500 (resp., 2,000).

\subsection{Summary}

Swift advance in hardware technology favors the evolution of WSNs toward serviceoriented networks and renders WSN-based service discovery a rising research issue. Some emerging service-oriented WSNs, for example, MSNs and WSANs, indicate the particular need in distance-sensitive service discovery algorithms. Although many generic service discovery approaches and adoptable techniques have been proposed for wireless ad hoc networks in the literature, they have weaknesses in message overhead and/or storage load and are thereby not a good option for WSNs with severe resource constraints.

In this chapter, we proposed a lightweight distance-sensitive service discovery protocol, iMesh. It is grounded on a novel localized planar structure, i.e., information mesh, with good proximity property. We analytically studied the properties of iMesh and show that it has very low message complexity and constant per node storage load. Through extensive simulation we evaluated the performance of iMesh in comparison with Quorum [48,72,74]. Simulation results confirm our theoretical findings and indicate that, iMesh nearly provide closest/nearby service selection guarantee with message overhead way smaller than Quorum. They indicate that iMesh-B outperforms iMesh-A in distance sensitivity practically at no cost. 


\section{CHAPTER 6 AUTONOMOUS SENSOR RELOCATION}

$\mathrm{N}$

ode failure is a common phenomenon that may induce coverage loss in WSNs. Deploying a large number of redundant sensors is the only way to preserve coverage against node failures in static WSNs; whereas, there exists an alternative solution, sensor relocation, in MSNs. To our knowledge, only two sensor relocation protocols WCP [79] and WCPZ [80] were proposed for coverage maintenance in the literature. They both lack node replacement guarantee and have many other major drawbacks. In this chapter, we propose two localized sensor relocation protocols, ZONER and MSRP, which are respectively applications of Quorum $[48,72,74]$ and iMesh (Chapter 5). The two protocols require no pre-knowledge of the sensory field or of the underlying network and guarantee efficient node replacement.

In Section 6.1, we review the literature at short length and briefly describe ZONER and MSRP; in Section 6.2, we define the network model; in Section 6.3, we introduce a restricted flooding technique, ZFlooding; in Sections 6.4 and 6.5, we present and ZONER and MSRP in detail; in Sections 6.6 and 6.7, we discuss how to deal with node failure and node contention during sensor relocation; in Section 6.8, we discuss the properties of ZONER and MSRP in comparison with WCP [80] and WCPZ [31]; in Section 6.9, we summarize the chapter. 


\subsection{Introduction}

Coverage is an important QoS factor in sensor networks. It is measured by the overall area that a sensor network is currently monitoring. The larger the coverage is, the better surveillance service the network can provide. As a sensor network operates, its coverage decreases because of runtime node failures. In order to maintain service quality, a sensor network must have the capability of preventing such coverage loss. In static sensor networks, using a large number of redundant nodes is the only way to preserve coverage in the face of node failures; relevant research concentrates mainly on how to schedule sensor activities to save energy without jeopardizing existing network coverage [29,81]. In MSNs, node mobility can be exploited to facilitate coverage maintenance, that is, it can be used to enable strategic and autonomous sensor movement for sensing hole filling.

At the time of writing, only two sensor relocation protocols WCP [79] and WCPZ [80] were proposed in the literature. They are both inferior for possible applications, compared to the protocols to be proposed in this chapter, for variety of reasons. WCP is a flooding-based protocol that generates inconstant migration delay and unbalanced energy usage, and may cause frequent topological change. $\mathrm{WCPZ}$ is a quasi-distributed algorithm that relies on preknowledge of the border of the sensory field and thus has limited applicability in real-world scenarios. They both use global or cross-network message transmissions for replacement discovery, generating $O\left(n^{\prime} \sqrt{n}\right)$ (or even $O\left(n^{\prime} n\right)$ ) messages, where $n^{\prime}$ and $n$ are respectively the number of redundant sensors and the number of non-redundant sensors; they both require inconstant per node storage load $O\left(n^{\prime}\right)$, and address no issue of guaranteed discovery of a replacement sensor when one in fact exists and is connected to the area where it could move.

Some sensor self-deployment algorithms, e.g., $[33,36,38,52,59,62]$, are adaptive to node failures and thus may be adopted to solve the sensor relocation problem. These algorithms aim at a uniform sensor distribution; once the uniformity is broken, they will reorganize a portion of or even the entire network to recover. Considering the fact that MSNs are failure-prone networks, such a sensor self-deployment algorithm will however lead to frequent change in 
network topology, complexing networking protocols and causing considerable energy loss.

\subsubsection{Our contributions}

In this chapter, we proposed two localized protocols, a ZONE-based sensor Relocation (ZONER) protocol and a Mesh-based Sensor Relocation Protocol (MSRP), with replacement guarantee. ZONER outperforms WCP [79] and WCPZ [80] for its localized and preknowledge free nature as well as its guaranteed closest node replacement; MSRP is yet advanced protocol that is superior to not only WCP and WCPZ but also ZONER for its considerably low message complexity and constant per node storage load. Thanks to its employed replacement discovery algorithm iMesh (refer to Chapter 5), MSRP ensures closest (nearby) node replacement with very high probability, larger than $97 \%$ (resp., 99\%).

ZONER is based on a new restricted flooding technique, ZFlooding, featured with voidarea penetration capability. Like WCPZ [80], it is also an application of protocol Quorum $[48,72,74]$. In ZONER, each redundant node registers with all the non-redundant nodes inside a vertical registration zone spanning the entire network. When a node fails, some of its neighbors inquiry all the non-redundant nodes inside a bounded horizontal request zone for redundant nodes. Because the request zone intersects with a number of registration zones, the non-redundant nodes in the intersection areas as recommender can provide the requester with redundant node information. Once a satisfactory and available redundant node is identified, it will be relocated in a shifted way along a natural migration path to replace the failed node, without changing network topology. Because no network-wide flooding is used, and because only selected nodes are required to move, ZONER is both bandwidth and energy efficient.

Figure 6.1, where big dots and small dots stand for redundant nodes and non-redundant nodes respectively, gives a general view of how ZONER works. In this example, after node $a$ fails, its westmost neighbor $w$ and its eastmost neighbors $e$ collaboratively find a nearest redundant node $v$ through a recommender node $c$; then, $v$ as the replacement of $a$ is relocated in a shifted manner to the position of $a$ along a natural migration path, which is the aggregation 


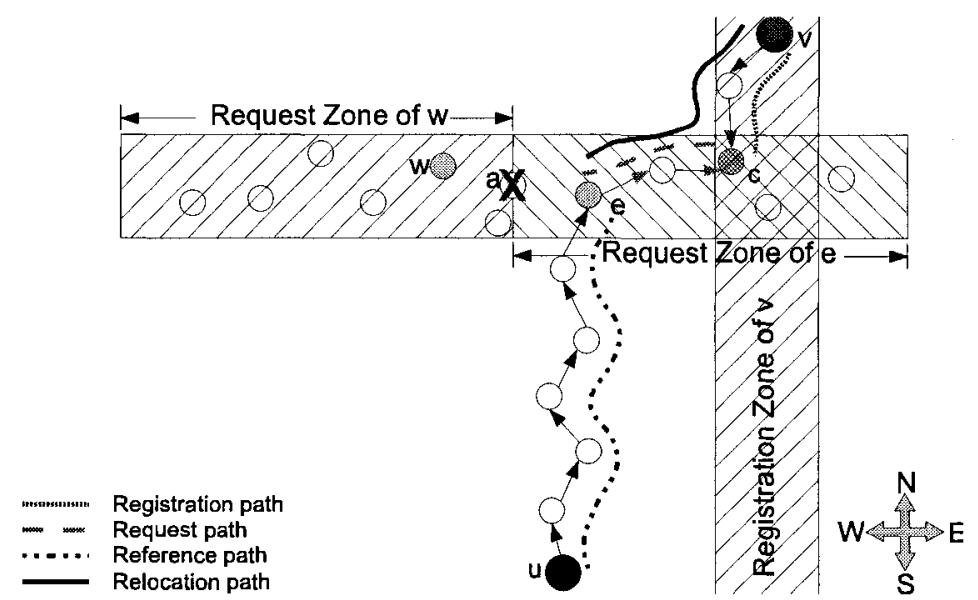

Figure 6.1: A general view of how ZONER works

of its registration path to the recommender $c$ and the request path of its discover $e$ to $c$.

MSRP is based on our previously proposed distance-sensitive service discovery algorithm iMesh. In MSRP, some non-redundant nodes are selected by redundant nodes as proxy and construct an information mesh over the network. The information mesh distributedly stores the location information of all the proxy nodes. Upon a node failure, the neighbors of the failed node find a nearby proxy node via the information mesh and take the proxy node's nearest delegated redundant node as the failed node's replacement. In order to generate constant relocation delay and balanced energy consumption, MSRP fulfills the replacement migration task by a shifted node relocation method. That is, it establishes a path between the failed node and the replacement node, and shifts all the nodes' position along the path toward the failed node. The novelty of this method stems from its localized energy-aware relocation path discovery mechanism that combines routing protocol GFG $[15,27]$ and the COST over PROGRESS ratio concept [73].

Figure 6.2 gives a general view of how MSRP works. In this figure, redundant nodes are represented by small solid circles; proxy nodes are denoted by big solid circles; the information mesh built by proxy nodes is highlighted by thick links. After a non-redundant node $a$ fails, its northmost, southmost, westmost, and eastmost non-redundant neighbors $n, s, w$, and $e$ collaborate to find a replacement, redundant node $r$ in this example, in $a$ 's vicinity. The 


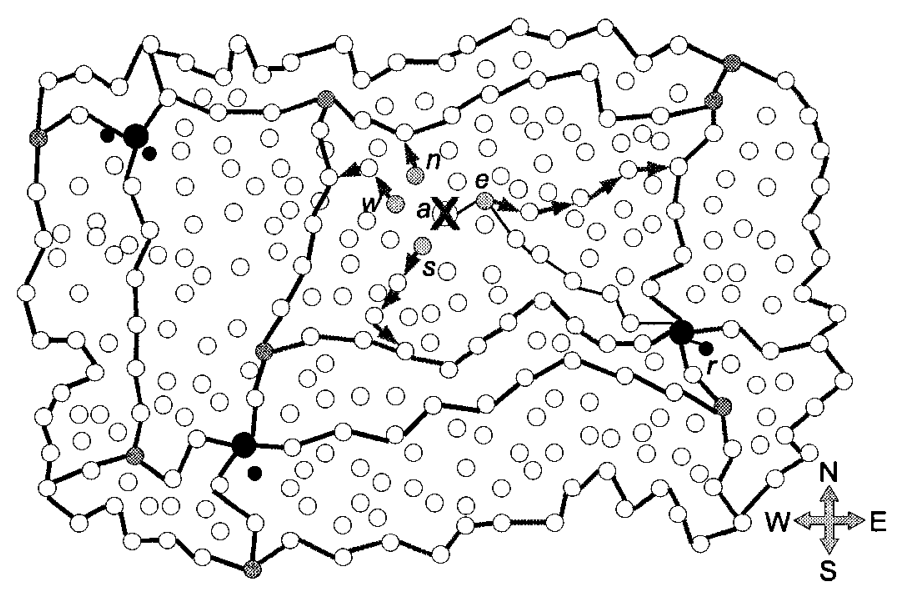

Figure 6.2: A general view of how MSRP works

paths along which the four nodes discover $r$ are shown by arrowed lines. The replacement node $r$ is then relocated to the position of failed node $a$ in a shifted way along the migration path marked by thin lines.

\subsection{Model and definitions}

We consider a connected MSN randomly deployed in plane, and assume the network has achieved a full coverage over the coverable area in the sensory field through a sensor selfdeployment algorithm. The nodes that constitute the network are non-redundant. They always remain active and thus are called active nodes (or A-nodes). We also assume that some predetermined redundant nodes (or R-nodes) are scattered in the network at random. R-nodes run a sleep/wakeup protocol to save energy and do not contribute to network connectivity. Both A-nodes and R-nodes stay static unless they are requested to move. Nodes are homogeneous. They are aware of their own location, which is an $(\mathrm{x}, \mathrm{y})$ coordinate, by a localization system like the Global Positioning System (GPS) [2] and have a two-hop neighborhood map through lower level protocols (minor modifications may apply). All the nodes use omni-directional antenna for communication, and their communication radius $r_{c}$ is at least twice as large as their sensing radius $r_{s}$. Any node may fail at any time for any reason. 
The our goal is to develop, based on the above network model, sensor relocation protocols that can maintain a network's coverage by relocating nearby R-nodes to the position of failed A-nodes. Note that, in a disconnected network, there is no guarantee that failed A-nodes are successfully replaced. For this reason, we additionally assume the network alway remains connected in spite of node failures. Furthermore, message collision and transmission errors can also affect the effectiveness of solution protocols. However, since these are MAC layer issues and beyond our scope, we assume reliable wireless communication channels so that we can concentrate on the sensor relocation problem itself.

\subsection{Range-restricted geographic flooding}

Flooding is a basic tool widely applied to a variety of network operations such as routing and service discovery. Because network-wide flooding involves the entire set of network nodes, its application is quite controversial in bandwidth-and/or energy- constrained networks like WSNs. For resource saving purpose, flooding ought to be restricted within a bounded range, which may be represented either by hop count or by geographic boundary.

A range-restricted geographic flooding algorithm could be as simple as follows: a node starts a flooding process by broadcasting a packet carrying the geographic border of the area to be flooded; a receiver node rebroadcasts the packet only when it is inside the specified flooding area; the flooding process terminates after all the nodes in the flooding area obtain the packet. But, if there is a void across the flooding zone, this simple algorithm may fail. Face routing is a proven effective building block for conquering the void problem in geographic routing [15,27]. In this section, we combine the above simple flooding algorithm with face routing and devise a range-restricted flooding technique, Zone Flooding (ZFlooding), featured with void-area penetration capability. This technique will be used later in Section 6.4 by protocol ZONER for node registration and discovery.

A node is said to be a local minimum in some direction (west, east, north, or south) if 


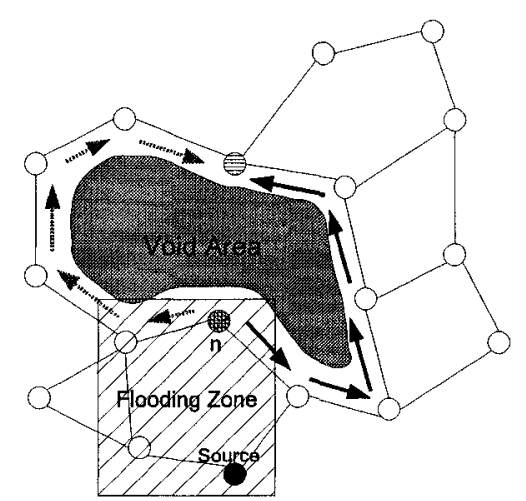

(a) The scenario of traversing an inner face.

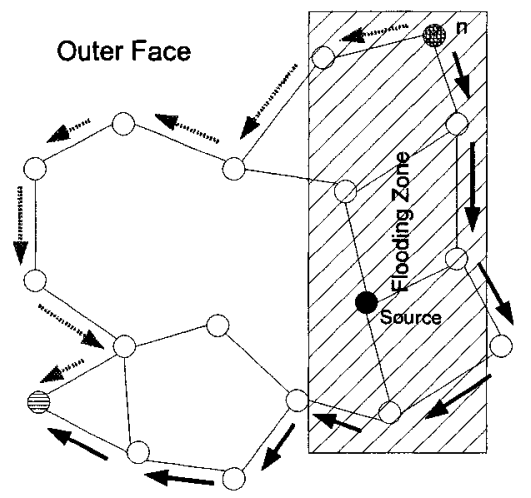

(b) The scenario of traversing the outer face.

Figure 6.3: An illustration of closed traversal circles

it is the foremost node in that direction in its one-hop neighborhood. For a local minimum $a$ in direction $d$, its two incidental edges, which respectively have the smallest angle and the largest angle with $d$, identify a particular face in the GG built on top of the underlying network. This face could contain a void area that stops the message transmission going through $a$ in direction $d$. To simplify expression, we call this face $d$-face. Before retransmitting a received ZFlooding packet $P k t$, node $a$ replicates it and stores the replica, denoted by $P k t^{\prime}$, locally. After retransmission, it attaches its own coordinate to $P k t^{\prime}$ and then sends two copies of $P k t^{\prime}$, one in the clockwise direction and the other in the counterclockwise direction, to traverse its $d$-face.

When a perimeter node $x$ receives $P k t^{\prime}$ for the first time, it checks if it itself is inside the flooding zone and is meanwhile more foremost than node $a$ in direction $d$, or if any of its neighbors satisfies the two conditions. If the answer is yes, it terminates the face traversal process as terminator, or otherwise forwards $P k t^{\prime}$ to the next perimeter node in the traversal direction as forwarder. During face traversal, if a forwarder node did not ever receive Pkt before, it also takes the following extra actions: restore $P k t$ by removing the coordinate of node $a$ from $P k t^{\prime}$ and broadcast $P k t$ locally.

If node $a$ is the foremost in direction $d$ in the flooding zone, its attempt to pass the ZFlood- 
ing packet $P k t$ around its $d$-face will end up with a closed traversal circle as shown in Figure 6.3. Observe that, if $a$ is by any chance the globally foremost in direction $d, P k t^{\prime}$ will actually traverse the outer face as displayed in Figure 6.3(b). In this case, provided each boundary node is aware of the fact that it itself is a boundary node, face traversal can be terminated earlier, and thus saving both bandwidth and energy. To enable this early termination, a separate (or, integrated) boundary detection process can be performed after GG construction.

\subsection{ZONER: a ZONE-based sensor Relocation protocol}

This protocol accomplishes the replacement discovery task and the replacement migration task respectively by the quorum technique $[48,72,74]$ and by the shifted migration method. All its involved flooding operations are range-restricted flooding accomplished by the ZFlooding technique. In the following, we will go through this protocol in detail.

At initiation, or when necessary, a R-node $v$ floods its registration zone by a registration message and registers with all the A-nodes inside the zone. The registration zone is a long narrow area centered at $v$ and vertically crossing the entire network; its width is a pre-defined the system parameter. During this registration process, an A-node in the registration zone may receive the registration message multiple times, each time along a different path. It maintains the backward shortest registration path of $v$ by remembering the neighbor from which it receives the message; it rebroadcasts the registration message if and only if the message comes along a path shorter than the one that it currently knows.

After an A-node $a$ fails, its westmost A-node neighbor $w$ and eastmost A-node neighbor $e$ independently start a discovery process by flooding their request zones with a request message. The request zone of $w(e)$ is a westbound (resp., eastbound) bounded area adjacent by $a$, as shown in Figure 6.1; its width is a pre-defined system parameter. The two nodes $w$ and $e$ are called server of the failed node $a$, and act as discovery partner of each other.

Consider server $e$. It first searches in its local memory for a registered R-node with shortest 
migration path, and then takes this node as reference to inquires all the A-nodes inside its request zone for a $\mathrm{R}$-node with yet shorter migration path to $a$. The migration path of the reference node is in fact its registration path. For message-saving purpose, the length of the request zone is set to be proportional to the length of the migration path of the reference node (if any) or infinity (otherwise). During the discovery process, every A-node in the request zone maintains a backward shortest request path of $e$. The request zone of $e$ intersects a number of

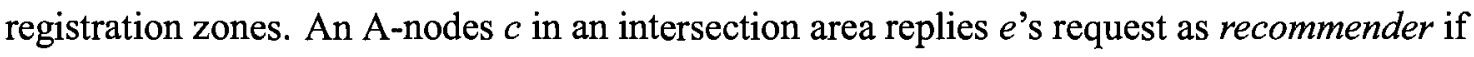
it has a registered $\mathrm{R}$-node whose migration path to $e$, i.e., the aggregation of the registration path of the R-node to $c$ and the request path of $e$ to $c$, is shorter than reference node's migration path to $e$. The reply messages is sent back to $e$ along previously established backward request path of $e$, meanwhile establishing the forward request path from $e$ to $c$.

Servers $w$ and $e$ perform replacement discovery independently. Finally, they exchange their discovery results and take the one with shortest migration path among all discovered $\mathrm{R}$-nodes as failure node $a$ 's replacement. The server that discovers the replacement is called replacement discoverer. Nodal relative position can be used to break ties. Figure 6.1 shows a big picture about a discovery process where $e$ is replacement discoverer.

Subsequently, replacement discoverer, say $e$, starts a migration process by sending a migration request along its request path to recommender $c$, which in turn forwards the message to replacement node $v$ along the backward registration path of $v ; v$ grants the request by sending an ACK message back to $e$ along the migration path; then it waits for an action message. Note that, as the migration request is transmitted from $c$ to $v$, the forward registration path of $v$ to $c$ is established. Having received the ACK message, $e$ sends an action message along the migration path to $v$, triggering nodes' physical movement. By shifted migration, $e$ moves to the position of $a$, while every other node along the migration path of $v$ moves to the position of its path neighbor toward $e$. Note that, before moving, the replacement node $v$ revokes its information in its registration zone using ZFlooding. In order to maintain information consistency and not to jeopardize the execution of other network protocols, every migrating node 
transfers all its local data to the newcomer at its original position immediately after.

\subsection{MSRP: a Mesh-based Sensor Relocation Protocol}

This protocol fulfills the replacement discovery task and the replacement migration task respectively by our previously proposed distance-sensitive service discovery algorithm iMesh (see Chapter 5) and by a novel shifted migration method. In the following, we will elaborate on its detail.

At initiation, each R-node spontaneously takes the nearest A-node as proxy, by sending that A-node a delegation request. In case of ties, nodal relative position can be used to help make decision. A R-node has one and only one proxy; whereas, a proxy is allowed to be shared by multiple R-nodes. Since proxy nodes locally record the location of their delegated R-nodes, unlimited R-node delegation may cause inconstant storage load on some A-nodes in case R-nodes are densely distributed. For instance, if all the R-nodes are located within the communication range of the same A-node, they may all chose this A-node as their proxy, yielding $O\left(n^{\prime}\right)$ storage load on it. In order to keep per node storage load constant, MSRP requires that an arbitrary A-node grant a delegation request from a R-node only when the number of its already delegated R-nodes is below a pre-defined threshold value. R-nodes whose delegation requests are rejected reissue delegation requests every time when they become awake. While being awake (by a wakeup/sleep protocol), a R-node monitors the liveness of its proxy; once it finds its proxy node fails, it moves to replace the proxy node directly.

Proxy nodes execute algorithm iMesh to construct an information mesh. Upon an ordinary (i.e., non-proxy) A-node failure, the A-nodes neighboring the failed A-node cooperate to discover a replacement, which is defined as the nearest delegated R-node of the target proxy of the failed A-node, by executing iMesh. For brevity, the target proxy (which is target service provider in iMesh) of a failed A-node is referred to as replacement proxy. During the replacement discovery process, the two lookup methods, i.e., cross lookup and perimeter lookup, may 
be selectively used, depending on requirement. For the cross lookup method, the northmost, the southmost, the eastmost and the westmost neighbors of the failed A-node, as servers, send four query messages respectively to the north, the south, the east, and the west direction, as shown in Figure 6.2. After receiving replies, they exchange their discovery results through underlaying routing protocol to find the replacement proxy. For the perimeter lookup method, only one neighbor, say the northmost neighbor, acts as the server of the failed A-node. It sends a query message to an arbitrarily selected direction, and later receives a reply that contains the replacement proxy's location. Whichever lookup method is employed, only the server that is closest to the replacement proxy is considered as replacement discoverer.

The replacement discoverer issues a migration request to the replacement proxy, which then grants the request by replying with an ACK message. In this hand-shaking process, message transmission is supported by routing protocol GFG [15, 27]. After receiving the $\mathrm{ACK}$, the replacement discoverer starts a migration path discovery process by sending an action message to the replacement proxy again by GFG, but in a different way.

Migration path discovery is in essence a QoS routing process. Its objective is to establish a path, between a replacement discoverer and a replacement proxy, which yields minimized energy usage and time delay for shifted migration. Recall that the shifted migration method requires all the node along a migration path to shift their position toward the failed A-node. From energy-saving point of view, node migration should involve shortest total moving distance and a least number of moves. In other words, a migration path is expected to have both minimized length and minimized hop count. On the other hand, to reduce migration latency in the case of simultaneous shifting, longest hop length in a migration path must be minimized, which is virtually equivalent to maximizing the hop count of the migration path. Under this contradictory circumstance, MSRP takes COST over PROGRESS ratio [73] as routing criterion and combines it with the greedy forwarding part of GFG during migration path discovery. Here, COST is defined as the Euclidean distance from current node to the considered next hop; PROGRESS is defined as the difference between the Euclidean distance from current node to 
the destination, i.e., a replacement proxy, and the Euclidean distance from the considered next hop to the destination. More formally, denote by $a_{0}$ the source, i.e., a replacement discoverer, and by $a_{i}$ the $i$-th hop along the path from $a_{0}$ to destination $d$. Then, the $(i+1)$-th hop $a_{i+1}$ must be closer to $d$ than $a_{i}$ and meanwhile minimize the following objective function:

$$
f\left(a_{i+1}\right)=\frac{\left|a_{i} a_{i+1}\right|}{\left|a_{i} d\right|-\left|a_{i+1} d\right|}
$$

Normally, a shifted migration process starts after the migration path is constructed. However, in order to save messages and reduce migration latency, in MSRP, node migration and migration path discovery are performed in parallel. More specifically, after sending the action message, the replacement discoverer moves to the failed node's location right away; intermediate nodes move to the position of their priori hop after forwarding the action message. The replacement proxy informs, after receiving the action message, the replacement node to fill its current position and then moves toward the location of its prior hop. In order not to jeopardize the information mesh structure or the execution of other network protocols, every migrating node transfers all its local data to the newcomer at its original position immediately after.

\subsection{Fault Tolerance}

Node failure can cause loss of control messages in replacement discovery and replacement migration. To tolerate such message loss, both ZONER and MSRP use a simple yet effective fault-tolerance approach, transmission retrial. During a replacement discovery process, if a server does not get any reply to its query message, it backs off for a while and retries. The server will receive a reply sooner or later, because any failed message forwarding A-node is going to be eventually replaced. However, in MSRP if the perimeter lookup method is applied, more care must be taken to avoid waiting loops. Fortunately, it is sufficient that, if an Anode participating a perimeter traversal finds that its next hop has failed, it immediately sends the query message back to the entry point. During a replacement migration process, if the 
replacement discoverer does not receive a reply (i.e., an ACK message) from the replacement proxy to its relocation request, it reissues the request. If, after a predefined number of trials, it still does not get any reply, it considers that the replacement node is unavailable and then tries to discover another one.

Run-time node failure may offset the effectiveness of ZONER and MSRP. Specifically, if all the default servers (i.e., the directional foremost A-node neighbors) of a failed A-node fail, or if the replacement discoverer fails, the failed A-node can not be replaced according to protocol design. Therefore, both ZONER and MSRP requires that, every other A-node neighbor of the failed A-node monitor the default servers via underlying routing protocol (i.e., by send them a prob message and expecting a reply) until the failed A-node is actually replaced. If a server fails during the monitoring period, the second foremost A-node neighbor in the corresponding direction takes over immediately. Note that, if some intermediate Anodes along a migration path fail in a node migration process executed for a failed A-node, the failed A-node is replaced by the replacement discoverer due to the nature of the shifted node migration method anyway, while those failed intermediate A-nodes will be locally identified (since they will never arrive their target position) later and eventually replaced with some other R-nodes.

\subsection{Contention Resolution}

Because of the distributed nature of ZONER and MSRP, node contention is very likely to happen during node migration. There are two types of node contention. Type-I is that multiple A-nodes are attempting to relocate the same R-node to replace different failure A-nodes; typeII is that an A-node appears in multiple migration paths and is required to shift its position along those paths. ZONER and MSRP handle the two types of node contention on a firstcome-first-serve basis. Recall that, a node migration process is triggered by a replacement discoverer after its migration request is granted by the replacement or replacement proxy. It is 
Table 6.1: Protocol comparison

\begin{tabular}{|l|c|c|c|c|}
\hline & WCP [79] & WCPZ [80] & ZONER & MSRP \\
\hline Protocol Classification & quasi-distributed & quasi-distributed & localized & localized \\
\hline ZERO Pre-knowledge & $\sqrt{ }$ & & $\sqrt{ }$ & $\sqrt{ }$ \\
\hline Guaranteed Node Replacing & & & $\sqrt{ }$ & $\sqrt{ }$ \\
\hline Constant Migration Latency & & $\sqrt{ }$ & $\sqrt{ }$ \\
\hline Constant Per-node Storage Load & & & & $\sqrt{ }$ \\
\hline Replacement Discovery Method & flooding & quorum & quorum & mesh \\
\hline Closest Replacement Discovery & & & $\sqrt{ }$ & \\
\hline Message Complexity & $O\left(n^{\prime} n\right)^{*}$ & $O\left(n^{\prime} \sqrt{n}\right)^{*}$ & $O\left(n^{\prime} \sqrt{n}\right)^{*}$ & $O(\psi(G))^{+}$ \\
\hline Node Migration Method & direct & shifted & shifted & shifted \\
\hline Guaranteed Migration Path Discovery & N/A & & $\sqrt{ }$ & $\sqrt{ }$ \\
\hline Energy-aware Migration Path & N/A & $\sqrt{ }$ & & $\sqrt{ }$ \\
\hline
\end{tabular}

" $n$ and $n$ ' denote the number of A-nodes and the number of R-nodes, respectively;

$+v+\sqrt{n} \leq \psi(G) \leq \operatorname{Min}\{v \sqrt{n}, n\}$, where $v \leq \operatorname{Min}\left\{n^{\prime}, n\right\}$ is \# of proxies, can be much less than $O(n)$ in order of magnitude when $v>\sqrt{n}$.

required that, once a R-node or a proxy node grants some A-node's migration request, it reject all the upcoming requests, preventing type-I node contention from happening. A replacement discoverer, whose migration request is rejected, backs off for a while and then tries to find some other R-node by a new replacement discovery process. An A-node in a migration path will start to relocate as soon as it forwards the corresponding action message. If the A-node also belongs to another migration path, then the decision on where it should move depends on which path it receives an action message first along. After moving, the A-node will transfer all the local data to the new comer at its original position, which then respond to buffered or upcoming action messages. By this means, type-II node contention is solved properly.

\subsection{Protocol Analysis}

In this section, we are going to analyze the characteristics of our new protocols ZONER and MSRP, and show their advantages in comparison with the two existing relocation proto- 
cols, i.e., WCP [79] and WCPZ [80].

Both MSRP and ZONER are a localized algorithm because they require sensors to know merely about their (two-hop) neighborhood information and do not involve any global computation like network-wide flooding or clustering; whereas, both WCP and WCPZ require certain centralized control (for mobile node management or for cluster management) and thus belong to the quasi-distributed algorithm category. Further, WCPZ relies on the pre-knowledge of the sensory field for grid formation. Considering scalability and applicability, the localized and pre-knowledge free protocols MSRP and ZONER are more desirable than the other two protocols WCP and WCPZ, especially for dense MSNs deployed in unknown environments.

Because the goal of a sensor relocation protocol is to maintain coverage, guaranteed node replacing is a crucial evaluation criterion. Both MSRP and ZONER employ the face routing technique to pass messages around void areas appearing in network topology. The void-area tolerance ability ensures replacement discovery and relocation path discovery, and therefore successful node replacing. On the contrary, because both WCP and WCPZ do not uses the face routing technique, they become problematic in the presence of void areas. Considering the guaranteed node replacing property, MSRP and ZONER defeat the other two protocols WCP and WCPZ already.

Considering the severe resource constraints of MSNs, a sensor relocation protocol is expected to generate constant per node storage load. MSRP does possess this property due to its application of algorithm iMesh, while the other three protocols including ZONER do not. In WCP, if all the mobile nodes are compactly located in a small area, some static nodes may be within the advertisement (flooding) range of every mobile node and thus have to store the information of all the mobile nodes. Similarly, in WCPZ and ZONER, if all the R-nodes are horizontally or vertically collinear, the A-nodes along a quorum may have to store the information of every single R-node. Thus, WCP, WCPZ and ZONER all require memory space $O\left(n^{\prime}\right)$ on each sensor node. The big difference in storage space requirement shows the unbeatable advantage of MSRP over the other relocation algorithms. 
In all the four protocols, majority of the messages are generated for replacement discovery. In a network with arbitrarily bad topology, both MSRP and ZONER can work probably with increased message overhead (due to the application of the face routing technique); the effectiveness of protocols WCP and WCPZ can however not be guaranteed. Under this circumstance, to have a clear and fair comparison on message overhead, we only consider a network with square-grid-like topology, where greedy forwarding always works. Hence, the message complexity of MSRP for replacement discovery can be expected to be within the range of $[O(v+\sqrt{n}), O(\operatorname{Min}\{v \sqrt{n}, n\})]$, where $v$ stands for the number of proxies, thanks to its employed iMesh algorithm. Those of protocols WCPZ and ZONER are expected to be $O\left(n^{\prime} \sqrt{n}\right)$ due to their quorum-based replacement discovery method. Protocol WCP uses a simple restricted flooding-based node discovery method, which is questionable because of the difficulty in the predefinition of the size of flooding areas. If the size of each flooding area is proportional to the size of the network, the message complexity of this method will be $O\left(n^{\prime} n\right)$. By above analysis, we can see that, from message complexity point of view, MSRP performs at least as well as the existing protocols and ZONER in the worst case but obviously better than them in average case.

Migration delay and energy consumption are important evaluation metrics. Protocol WCP moves mobile nodes directly to sensing holes, while protocols MSRP, WCPZ and ZONER all relocates a replacement node to the location of a failed A-node in a shifted manner. By the direct method, migration distance can be as bad as the spatial diameter (bounded by $O(n)$ ) of the network. Therefore, WCP can cause the battery power of a mobile node over-consumed and generate inconstant relocation delay. By the shifted method, all the the nodes along a relocation path shift their position toward a failed A-node. In this process, a node's moving distance is always bounded by its communication radius $r_{c}$, a constant value. Hence, MSRP, WCPZ and ZONER have balanced energy consumption and constant relocation delay (in the case of simultaneous shifting). Mentionably, although these three protocols use a similar shifted migration method, the difference between the ways that they discover a migration 
path make them actually perform differently. MSRP uses an advantageous localized routing mechanism to establish a migration path between a replacement and a failed A-node, while WCPZ employs a undesired flooding-based routing mechanism to do so. ZONER integrates migration path discovery within replacement discovery processes for message-saving purpose but without energy-saving consideration.

A replacement selected by ZONER for a failed A-node is always a R-node geographically closest to the failed A-node, while this may not be the case for WCP, WCPZ and MSRP. In WCP, void areas, for example, due to physical obstacles, can block the advertisement of mobile sensors, causing the loss of the closest replacement property. For the same reason, WCPZ may fail to find the nearest R-node as replacement for a failed A-node. In MSRP, closest proxy node selection is guaranteed with high probability larger than $96 \%$ according to siumation. For WCP, the lack of the closest replacement property is indeed a major drawback because the direct migration method that it uses is vulnerable to Euclidean distance. However, for WCPZ and MSRP, we do not consider so, because the shifted node migration method employed by them weakens the gravity of Euclidean distance. In particular, it is a tradeoff with all the other nice properties for MSRP.

Table 6.1 comparatively lists the characteristics of the four relocation protocols MSRP, WCP, WCPZ and ZONER. Note that, to have fair comparison, message complexity is only for a network with square-grid-like topology, where greedy forwarding always works.

\subsection{Summary}

In this chapter, we presented two localized sensor relocation protocols: ZONER and MSRP. As WCP, but unlike WCPZ, ZONER does not require any pre-knowledge of the sensory field or of the network; As WCPZ, but unlike WCP, ZONER employs the shifted migration method that yields bounded migration delay and balanced energy usage. Unlike both WCP and WCPZ, ZONER does not rely on global computation or communication, and it 
guarantees closest replacement discovery and node replacing with no concern with void areas (e.g., due to node failures or unbalanced node distribution). This guarantee stems from a restricted flooding technique ZFlooding, which combines simple geographic range-bounded flooding with the face routing technique.

MSRP is an application of protocol iMesh. Like ZONER, it is also a pre-knowledge free algorithm. It aims to replace failed sensors with nearby redundant ones through autonomous nodal movement. Its novelty exists in not only the use of iMesh but its purely localized energyaware migration path discovery mechanism, which combines GFG [15,27] with the concept of COST over PROGRESS ratio [73]. MSRP is superior to WCP and WCPZ as well as ZONER in message complexity and per node storage load. It guarantees replacement discovery and node replacing, which are both however not addressed by WCP and WCPZ. 
CHAPTER 7

CONCLUSIONS

$\Lambda_{\text {shallenges of traditional static WSNs are now embrassing new possibilities and ideas. }}^{\text {s nodes obtain controlled mobility, WSNs appears in a form as MSNs. Many design }}$ Among many important MSN-based research problems, we particularly emphasize on the problem of mobility-controlled coverage improvement in this thesis. We comprehensively and detailedly surveyed existing solutions, and pinpointed and solved two new research problems. We as well presented two new sensor relocation protocols, each of which has its own beauty and exhibits significant advantage over existing ones. In the following, we will summarize the contributions of this thesis and briefly discuss our future work.

\subsection{Sensor self-deployment}

We pinpointed a new sensor self-deployment problem, focused coverage formation, and defined an evaluation metric. We then proposed two solution algorithms GA and GRG. The two algorithms are the first in the literature that operate in a strictly localized manner and are yet able to provide coverage guarantee. Due to their localized nature, they are resilient to node failures and able to work regardless of network partition. Both GA and GRG are capable of generating a network of an equilateral tessellation layout and with hole-free coverage. 
We presented two versions of GRG, which respectively ensure optimal hexagonal coverage radius and optimal circular coverage radius. We proved the correctness of GA and GRG and evaluated their performance through extensive simulation.

\subsubsection{Open Problems}

In this thesis, we have been in the simple context where there is a single geographic point as the POI. Complex scenarios exist in reality. For instance, there are a series of continuous POIs forming a Line of Interest (LOI), representing an object like the trace of certain event or the border of a landmark (e.g., a building). A yet complex and general scenario is that there are multiple discrete target objects, each of which is abstracted either as a POI or a LOI.

GA does not provide coverage radius guarantee, and the connectivity of its resulting network relies on the occupancy of the POI. These two shortcomings restricts the applicability of GA. Contrarily, GRG can be applied to every case and always guarantees optimal coverage radius. It is extendable to the multi-POI scenarios mentioned above. However, the extension is not trivial and will be part of our future work. Below we will discuss how it can be done in a nutshell to show feasibility.

\section{Line of Interest (LOI)}

To form a focused coverage around a LOI, the entire LOI must be given as input to every sensor. An arbitrary sensor first takes a point, say the north-east most point, on the LOI as reference and then construct a TT graph. Then, it finds the smallest set of successive TT vertices that best represent the LOI. These vertices constitute a coverage backbone. All the sensors must apply the same set of rules so that they can construct the same TT graph and the same coverage backbone. Once the coverage backbone is established, sensors may greedily move towards or rotate around it following the same philosophy as GRG. Note that, if the LOI is a closed line, its inside will not be covered by any sensor. This is reasonable, for example, in the case that sensors are required to surround a building, a lake or a hill. 


\section{Multiple target objects}

Assume that there are $n$ number of target objects, each of which is represented either by a POI or by a LOI. Sensors assign themselves to the $n$ objects automatically and take care of their assigned object by running GRG. Since sensors know how to surround a POI or a LOI, the problem that they are facing now is how to perform self-assignment. And, because no global knowledge is available, it must be solved through a probabilistic approach. Note that, with small modification, this extension can be applied to the situation where the deployment field contains physical obstacles.

Suppose that it is sufficient from QoS viewpoint for any object to have a focused coverage with hole distance equal to $\frac{3}{2} r_{s} q$. In other words, an object is expected to be surrounded by $q$ number of hexagons of sensors. How many sensors that an object needs depends on the size of the object. The larger the size, the more sensors needed. Consider two objects with separation less than $2 q$-hexagon distance. They will share the sensors in between so as to make a twofold benefit: saving sensors and reducing inter-sensor signal interfere, causing the decrease of the total number of their demanded sensors. Hence, sensor assignment must consider both object size and object separation.

Now, we are ready to give a simple localized probabilistic sensor self-assignment method. Sensors group the $n$ objects into $n^{\prime}$ clusters according to their separation and the parameter $q$ and treat every cluster $C_{t}\left(0 \leq t<n^{\prime}\right)$ as an integrated object. For every $C_{t}$, they compute the number $r_{t}$ of sensors for surrounding it in $q$ hexagons, estimate the number $g_{t}$ of sensors for covering the gaps between its member objects, and get the total number $m_{t}=r_{t}+g_{t}$ of sensors needed. Afterwards, sensors compute their probability $p_{t}=\frac{m_{t}}{\sum_{0 \leq i<n^{\prime}} m_{i}}$ of being assigned to each $C_{t}$ and chose a cluster with corresponding assignment probability. The whole process is local computation with no message transmission. 


\subsection{Service discovery}

The emergence of service oriented robotic WSNs such as MSNs and WSANs gives rise of a new research problem, distance-sensitive service discovery, which is formalized in this thesis for the first time. We proposed a localized solution algorithm iMesh that employs a novel localized planar structure information mesh with good proximity property as service directory. With the help from information mesh, service lookup in iMesh is restricted within certain bounded (usually small) search cell and is performed also in a purely localized. That is, each service consumer searches in four directions within its residing home cell(s) and is guaranteed to discover a nearby service provider once hitting its home cell perimeter. We analyzed the properties including construction cost and distance sensitivity of iMesh in depth. Our study shows that iMesh has very low message complexity and preferred constant per node storage load; our extensive simulation verified our finds and indicated that iMesh guarantees closest (nearby) service discovery with very high probability larger than $96 \%$ (resp., 99\%).

\subsubsection{Open problems}

\section{Extension to multi-service scenarios}

In this thesis, iMesh was presented under the assumption of single-service networks, which is however not a common setting in practice. When there is more than one type of service provided in the network, a multi-layered information mesh can be constructed to support service discovery. That is, the same type of service providers together constitute a mesh layer, and different layers correspond to different types of services. For a network with $k \geq 1$ service types, the height, i.e., the number of layers, of the information mesh is equal to $k$. Note that a node offering multiple types of services will appear in more than one layer of the information mesh. Fig. 7.1 shows an information mesh of three layers. In this figure, SP-node $p$ offers all the three type of services and thus exists in every single layer of the information mesh.

With cares, the message complexity of constructing a multi-layered information mesh can 

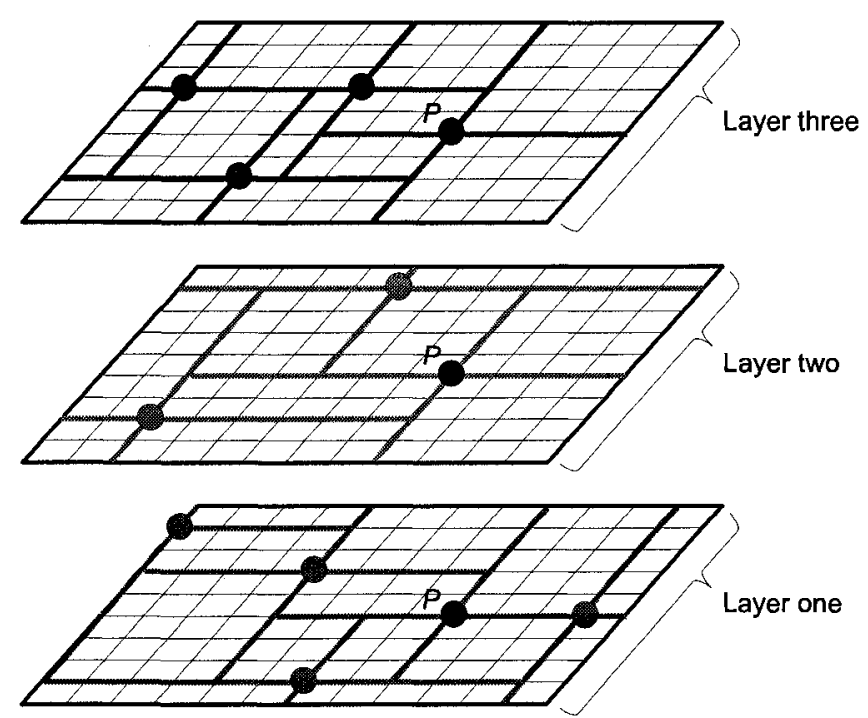

Figure 7.1: A 3-tier information mesh

be made not larger than the summation of the message complexity of building every single mesh layer separately. For instance, a SP-node shared by $t$ number of mesh layers does not necessarily distribute its location information $t$ times. Instead, it attaches $t$ bits to the location information to indicate its offered services. The information is virtually blocked in one layer by flipping the corresponding bit; it physically stops propagating when all the attached bits are flipped or when it reaches the network border. By this means, the SP-node fulfills its construction duty in all its residing layers simultaneously, thus saving a considerable number of messages. An obvious coarse upper bound of the message complexity is $v \sqrt{n}$.

With the multi-layered information mesh, when a node wants to discover a particular type of service, it just needs to perform cross service lookup in the corresponding layer as if it is still in a single-service network. In this way, the distance sensitivity and the service lookup message overhead of iMesh naturally stay unchanged. Because a node shared by $t(1 \leq t \leq k)$ mesh layers has to store a constant amount of information for each of its residing layer (by Theorem 5.3), it has $O(t) \leq O(k)$ storage load in total. Since $k$ is usually a known value at the network deployment time, iMesh still yields constant per node storage load. 


\section{Guarantee on nearby service selection}

Since remote service selection is still possible, with very low possibility though, modifying the information mesh structure, or developing other structure (e.g., triangular mesh), to achieve nearby service selection guarantee will be our possible future work.

\subsection{Sensor relocation}

In this thesis, we presented two sensor relocation protocols ZONER and MSRP. Protocol ZONER is a variant of the quorum-based location service $[48,72,74]$. It uses a restricted flooding technique, ZFlooding, for quorum formation and adopts the shifted method for replacement migration. Compared with an existing quorum-based sensor relocation protocol WCPZ [80], ZONER has the following advantages: it requires no knowledge of the sensory field such as the geographic border as a priori and thus has strong availability to unknown environments; it uses no global computation such as clustering, and its replacement discovery is performed in a localized manner using solely local knowledge; thanks to the void-area penetration property of ZFlooding, it is able to provide guarantee on closest replacement discovery with no concern with obstacles or unbalanced node distribution.

Protocol MSRP is a yet advanced localized pre-knowledge free sensor relocation protocol with novelty in both replacement discovery and in replacement migration. For replacement discovery, MSRP employs our proposed distance-sensitive service discovery algorithm, iMesh, whose novelty and efficiency have be summarized previously. As for replacement migration, MSRP adopts the shifted method along with a novel migration path discovery scheme that combines GFG routing technique [15,27] with the COST over PROGRESS ratio [73]. By this means, it yields energy-aware migration path in a purely localized way. A comparison of WCP, WCPZ, ZONER and MSRP can be found in Table 6.1 in Chapter 6 . 


\subsubsection{Open problems}

Despite the clear hints on their behaviors, ZONER and MSRP are both lacking simulation study. In the future, we are going to implement these two algorithms and empirically evaluate their performance in comparison with WCPZ [80]. Certainly, looking for other more effective and efficient relocation protocols will also be part of our future work. 


\section{APPENDIX A}

COMPLEMENTARY RESULTS

\section{A.1 Intersection transformation}

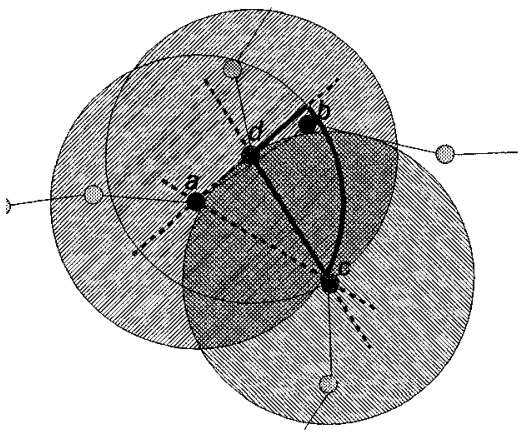

(a) $a$ is not neighboring $c$ but $d$

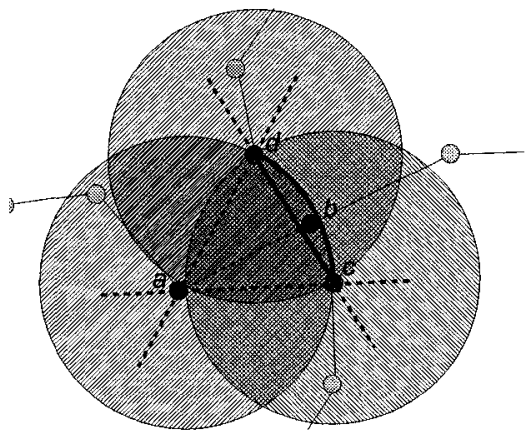

(b) $a$ is neighboring neither $c$ nor $d$

Figure A.1: A pair of crossover links $a b$ and $c d$

In the following, we show that a link-crossing case can be locally transformed to a nodesharing case in a sensor network modeled as a unit disk graph. Consider a pair of crossover links $a b$ and $c d$. We need to show that one of $a b$ and $c d$ has an end node neighboring the two end nodes of the other link. Randomly take one node from each link, say $a$ from $a b$ and $c$ from $c d$. If $a$ is adjacent to both $c$ and $d$, then we are done. Let us assume, without loss of generality, that $a$ is not neighboring $c$, as illustrated in Fig. A.1. 


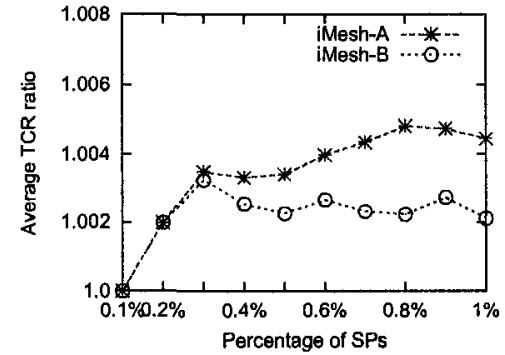

(a) Average TCR with small PSP

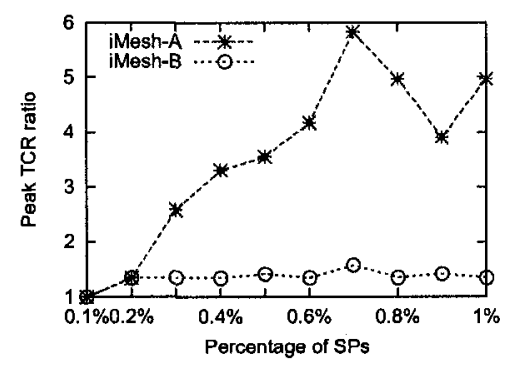

(c) Peak TCR with small PSP

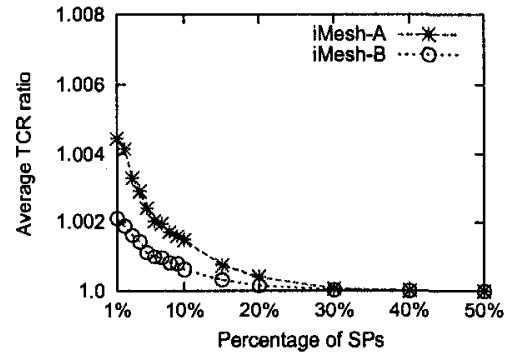

(b) Average TCR with large PSP

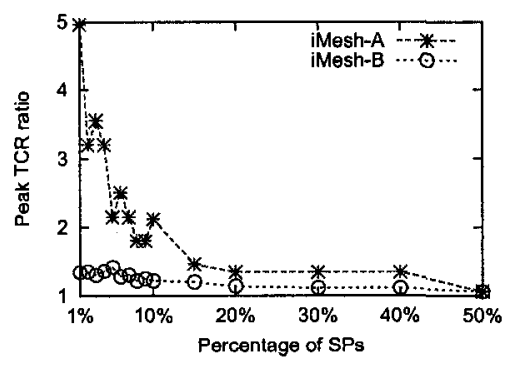

(d) Peak TCR with large PSP

Figure A.2: TCR in a WSN of 1,000 nodes

In order for $a b$ to crosses $c d, b$ must satisfy the following conditions: (1) it is located on the different side of $\overline{c d}$ from $a$ or on $\overline{c d}$ between $c$ and $d$; (2) it is located on $\overline{a d}$ or on the same side of $\overline{a d}$ as $c$; (3) it is located on $\overline{a c}$ or on the same side of $\overline{a c}$ as $d$; (4) it is within the transmission range of $a$. In Fig. A.1, the residence area of $b$ is enclosed (inclusively) by solid thick line. It is provable that, if $a$ is neighboring $d$, then $b$ must be neighboring $d$, or both $c$ and $d$ otherwise.

\section{A.2 iMesh in a WSN of 1,000 nodes}

Observe the experimental results depicted in Figures A.2 and A.3 Figures and those given in Figure 5.13. It is not difficult to find that iMesh has consistent distance sensitivity performance in different sized networks.

Note: Figures A.2 and A.3 indicate that iMesh has best distance sensitivity, i.e., TCR $=1$ and PTCR1 $=1(\mathrm{PTCR} 2=\mathrm{PTC} 3=0)$, when PSP $=0.1 \%$ in a WSN of size 1,000 . It is 


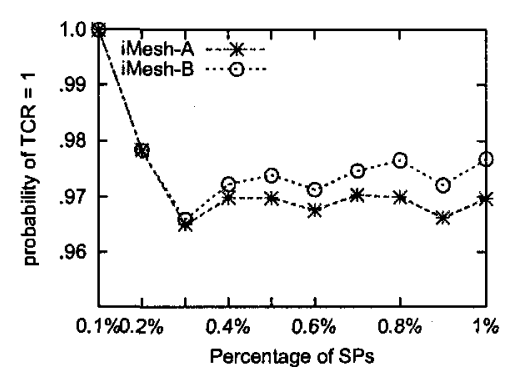

(a) PTCR1 with small PSP

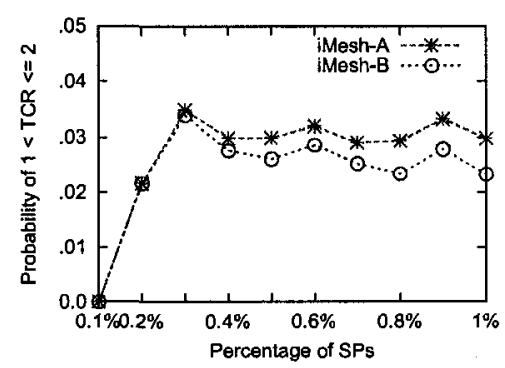

(c) PTCR2 with small PSP

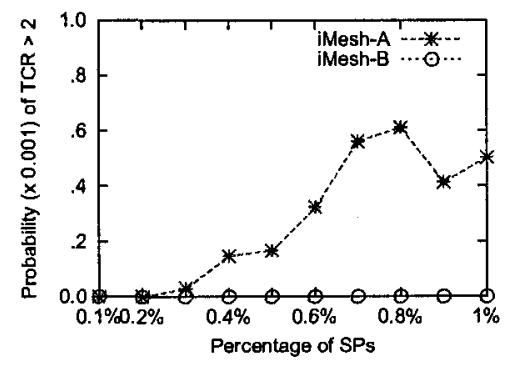

(e) PTCR3 $\left(\times 10^{-3}\right)$ with small PSP

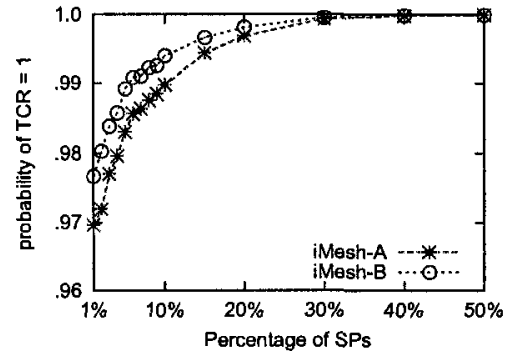

(b) PTCR1 with large PSP

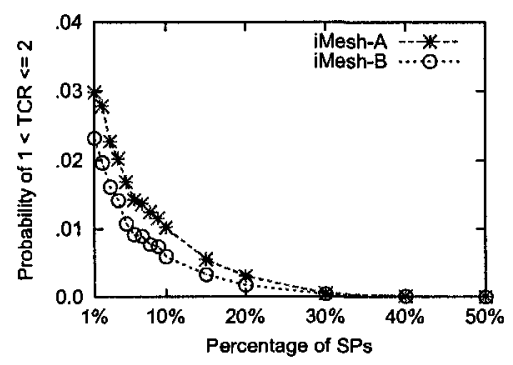

(d) PTCR2 with large PSP

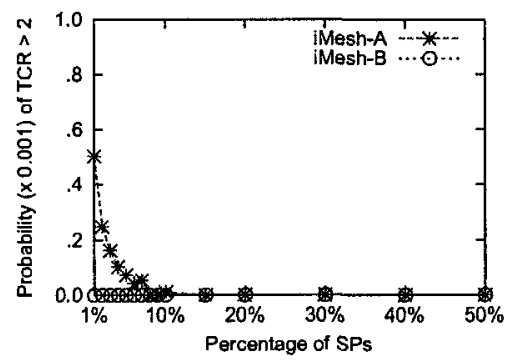

(f) PTCR3 $\left(\times 10^{-3}\right)$ with large PSP

Figure A.3: PTCR in a WSN of 1,000 nodes

indeed true since, in that case, the network contains only one SP that is naturally closet to every SC. 


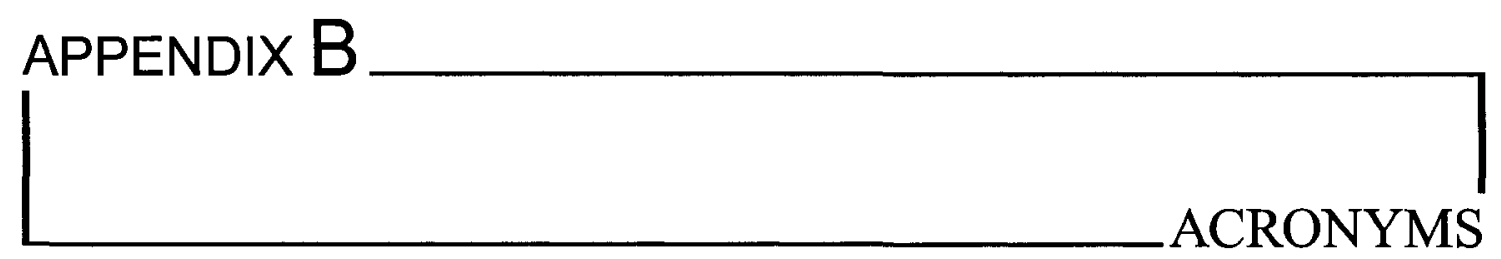

A-node: Active node

CPU: Central Processing Unit

CT: Convergence Time

CV: Collision aVoidance

CW: Collision alloWance

ECD: Essential Coverage Disc

FIFO: First-In, First-Out

GA: Genetic Algorithm

GA: Greedy Advance

GFG: Greedy-Face-Greedy

GG: Gabriel Graph

GPS: Global Positioning System

GRG: Greedy-Rotation-Greedy

IMLM: Intelligent Mobile Land Mines 
LOI: Line of Interest

MANET: Mobile Ad hoc Network

MG: Mileage

MP: Mileage over Progress

MSN: Mobile Sensor Networks

MSRP: Mesh-based Sensor Relocation Protocol

NC: Number of Collisions

NCMSP: Number of Construction Messages per Service Provider

NM: Number of Moves

NSMSC: Number of Search Messages per Service Consumer

PDOCC: Proportional Difference of Construction Cost

PG: Progress

POI: Point of Interest

PSP: Percentage of Service Providers

PTCR1: Probability of TCR $=1$

PTCR2: Probability of $1<\mathrm{TCR} \leq 2$

PTCR3: Probability of TCR $>2$

QoS: Quality of Service

RF: Radio Frequency

RNG: Relative Neighborhood Graph

ROI: Region of Interest

R-node: Redundant node

SC: Service Consumer

SP: Service Provider 
SPV: Set of Providers in Vicinity

SZ: Size

TCR: Target over Closest Ratio

TNCM: Total Number of Construction Messages

TT: Equilateral Triangle Tessellation

WSN: Wireless Sensor Network

WSAN: Wireless Sensor and Actor Network

ZONER: ZONE-based sensor Relocation 
REFERENCES

[1] Intelligent Mobile Land Mines (IMLM). Sandia National Laboratories. http://www . arpa.mil/STO/smallunitops/SHM/sandia.html.

[2] Global Positioning System Standard Positioning Service Signal Specification, 2nd Edition, June 1995. http://www.navcen. uscg.gov/pubs/gps/sigspec/gpssps1.pdf.

[3] I. Abraham, D. Dolev, and D. Malkhi. "LLS: a Locality Aware Location Service for Mobile Ad Hoc Networks". In Proceedings of the Joint Workshop on Foundations of Mobile Computing (DIALM-POMC), pages 75-84, 2004.

[4] N. Ahmed, S. Kanhere, and S. Jha. "Probabilistic coverage in wireless sensor networks". In Proceedings of IEEE Workshop on Wireless Local Networks (WLN), pages 672-681, 2005.

[5] K. Akkaya and M. Younis. "A survey on routing protocols for wireless sensor networks". Ad Hoc Networks, 3(3):325-349, 2005.

[6] I. Akyildiz and I. Kasimoglu. "Wireless sensor and actor networks: Research challenges". Ad Hoc Networks, 2(4):351-367, 2004.

[7] I. Akyildiz, W. Su, Y. Sankarasubramaniam, and E. Cayirci. "Wireless sensor networks: a survey". Computer Networks, 38(4):393-422, 2002. 
[8] J. N. AL-KARAKI and A. E. KAMAL. "Routing techniques in wireless sensor networks: a survey". IEEE Wireless Communications Magazine, 11(6):6-28, 2004.

[9] F. Aurenhammer and R. Klein. "Voronoi Diagrams". http://www. pi6. fernuni-hagen . de/publ/tr198.pdf.

[10] J. Bachrach and C. Taylor. "Localization in sensor networks". In I. Stojmenovic, editor, Handbook of Sensor Networks, chapter 9, pages 277-310. Wiley, 2005.

[11] X. Bai, S. Kumary, D. Xuan, Z. Yun, and T. H. Lai. "Deploying Wireless Sensors to Achieve Both Coverage and Connectivity". In Proceedings of the 7th International Symposium on Mobile Ad Hoc Networking and Computing (MobiHoc), pages 131-142, 2006.

[12] S. Basagni, M. Conti, S. Giordano, and I. Stojmenovic. "Mobile Ad Hoc Networking". IEEE Press, 2004.

[13] M. A. Batalin and G. S. Sukhatme. "The Analysis of an Efficient Algorithm for Robot Coverage and Exploration based on Sensor Network Deployment". In Proceedings of IEEE International Conference on Robotics and Automation (ICRA), pages 3478-3485, 2005 .

[14] S. Bergbreiter and K. Pister. "Cotsbots: An off-the-shelf platform for distributed robotics". In Proceedings of IEEE/RSJ International Conference on Intelligent Robots and Systems (IROS), pages 1632-1637, 2003.

[15] P. Bose, P. Morin, I. Stojmenovic, and J. Urrutia. "Routing with Guaranteed Delivery in Ad Hoc Wireless Networks". In Proceedings of ACM DIALM (LNCS 4325), pages $48-55,1999$.

[16] W. Burgard, M. Moors, D. Fox, R. Simmons, and S. Thrun. "Collaborative multi-robot exploration". In Proceedings of IEEE International Conferenceon Robotics and Automation (ICRA), pages 476-481, 2000. 
[17] E. H. Callaway. “The Wireless Sensor Network MAC". In I. Stojmenovic, editor, Handbook of Sensor Networks, chapter 8, pages 239-276. Wiley, 2005.

[18] S. Chellappan, X. Bai, B. Ma, and D. Xuan. "Mobility Limited Flip-Based Sensor Networks Deployment”. IEEE Transactions on Parallel and Distributed Systems, 18(2):199-211, 2007.

[19] W.-P. Chen and J. Hou. "Data gathering and fusion in sensor networks". In I. Stojmenovic, editor, Handbook of Sensor Networks, chapter 15, pages 493-526. Wiley, 2005.

[20] K. Dantu, M. Rahimi, H. Shah, S. Babel, A. Dhariwal, and G. S. Sukhatme. "Robomote: Enabling Mobility in Sensor Networks". In Proceedings of the 4th IEEE/ACM International Conference on Information Processing in Sensor Networks (IPSN), pages 404 409, 2005.

[21] J. Elson and D. Estrin. Wireless sensor networks: A bridge to the physical world. In C. S. Raghavendra, K. M. Sivalingam, and T. Znati, editors, Wireless Sensor Networks, chapter 1, pages 3-20. Kluwer, 2004.

[22] Q. Fang, J. Gao, and L. J. Guibas. "Landmark-Based Information Storage and Retrieval in Sensor Networks". In Proceedings of the 25th IEEE International Conference on Computer Communications (INFOCOM), pages 286-297, 2006.

[23] Q. Fang, J. Gao, L. J. Guibas, V. Silva, and L. Zhang. “GLIDER: Gradient LandmarkBased Distributed Routing for Sensor Networks". In Proceedings of the 24th Annual Joint Conference of the IEEE Computer and Communications Societies (INFOCOM), pages 339-350, 2005.

[24] C. Frank and H. Karl. "Consistency Challenges of Service Discovery in Mobile Ad Hoc Networks". In Proceedings of the 7th ACM International Symposium on Modeling, Analysis and Simulation of Wireless and Mobile Systems (MSWiM), pages 105-114, 2004. 
[25] J. Fredslund and M. J. Mataric. "Robot formations using only local sensing and control”. In Proceedings of IEEE International Symposium on Computational Intelligence in Robotics and Automation (CIRA), pages 308-313, 2001.

[26] H. Frey and I. Stojmenovic. "Geographic and Energy aware Routing in Sensor Networks". In I. Stojmenovic, editor, Handbook of Sensor Networks, chapter 12, pages 381-415. Wiley, 2005.

[27] H. Frey and I. Stojmenovic. "On Delivery Guarantees of Face and Combined GreedyFace Routing Algorithms in Ad Hoc and Sensor Networks". In Proceedings of ACM MobiCom, pages 390-401, 2006.

[28] R. Friedman and G. Kliot. "Location Services in Wireless Ad Hoc and Hybrid Networks: A Survey". Technical Report CS-2006-10, Department of Computer Science, Technion, Haifa, Israel, April 2006.

[29] A. Gallais, J. Carle, D. Simplot-Ryl, and I. Stojmenovic. "Localized Sensor Area Coverage with Low Communication Overhead". In Proceedings of the 4th Annual IEEE International Conference on Pervasive Computing and Communications (PerCom), pages $328-337,2006$.

[30] Z. Gao, L. Wang, M. Yang, and X. Yang. "CNPGSDP: An Efficient Group-based Service Discovery Protocol for MANETs". Computer Networks, 50(16):3165-3182, 2006.

[31] Z. Gao, L. Wang, M. Yang, and X. Yang. "CNPGSDP: An Efficient Group-based Service Discovery Protocol for MANETs". Computer Networks, 50(16):3165-3182, 2006.

[32] Z. Gao, Y. Yang, J. Zhao, J. Cui, and X. Li. "Service Discovery Protocols for MANETs: A Survey". In Proceedings of the 2nd International Conference on Mobile Ad-hoc and Sensor Networks (MSN) (LNCS 4325), pages 232-243, 2006. 
[33] M. Garetto, M. Gribaudo, C.-F. Chiasserini, and E. Leonardi. "A Distributed Sensor Relocation Scheme for Environmental Control". In Proceedings of the 4th IEEE International Conference on Mobile Ad-hoc and Sensor Systems (MASS), 2007.

[34] M. Hefeeda and H. Ahmadi. "A Probabilistic Coverage Protocol for Wireless Sensor Networks". In Proceedings of the 15th IEEE International Conference on Network Protocols (ICNP), pages 41-50, 2007.

[35] S. Helal, N. Desai, V. Verma, and C. Lee. "Konark - A Service Discovery and Delivery Protocol for Ad-Hoc Networks". In Proceedings of IEEE Wireless Communications and Networking Conference (WCNC), pages 2107-2133, 2003.

[36] N. Heo and P. K. Varshney. "Energy-Efficient Deployment of Intelligent Mobile Sensor Networks". IEEE Transactions on Systems, Man, and CyberNetics - Part A: Systems and Humans, 35(1):78-92, 2005.

[37] A. Howard, M. J. Mataric, and G. S. Sukhatme. "An Incremental Self-Deployment Algorithm for Mobile Sensor Networks”. Autonomous Robots, 13(2):113-126, 2002.

[38] A. Howard, M. J. Mataric, and G. S. Sukhatme. "Mobile Sensor Network Deployment using Potential Fields: A Distributed, Scalable Solution to the Area Coverage Problem". In Proceedings of the 6th International Symposium on Distributed Autonomous Robotics Systems (DARS), 2002.

[39] M. Klein and B. K. Ries. "Multi-layer Clusters in Ad Hoc Networks - an Approach to Service Discovery". In Proceedings of Networking Workshops on Web Engineering and Peer-to-Peer Computing (LNCS 2376), pages 99-110, 2002.

[40] M. Klein, B. K. Ries, and P. Oberiter. "Service rings - a Semantic Overlay for Service Discovery in Ad Hoc Networks". In Proceedings of th 14th IEEE International Workshop on Database and Expert Systems Applications (DEXA), pages 180-185, 2003. 
[41] U. C. Kozat and L. Tassiulas. "Service Discovery in Mobile Ad Hoc Networks: an Overall Perspective on Architectural Choices and Network Layer Support Issues". Ad Hoc Networks, 2(1):23-44, 2004.

[42] F. Kuhn, R.Wattenhofer, Y. Zhang, and A. Zollinger. "Geometric Ad Hoc Routing: Of Theory and Practice". In Proceedings of ACM PODC, pages 63-72, 2003.

[43] B. Leong, S. Mitra, and B. Liskov. 'Path Vector Face Routing: Geographic Routing with Local Face Information". In Proceedings of 13th IEEE International Conference

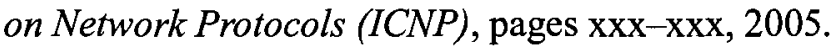

[44] J. Li, J. Jannotti, D. S. J. D. Couto, D. R. Karger, and R. Morris. “A Scalable Location Service for Geographic Ad Hoc Routing". In Proceedings of the 6th Annual ACM International Conference on Mobile Computing and Networking (MobiCom), pages 120-130, 2000.

[45] X. Li, H. Frey, N. Santoro, and I. Stojmenovic. "Localized Sensor Self-deployment with Coverage Guarantee". ACM Mobile Computing and Communications Review, 2008. To appear.

[46] X. Li, Y. J. Kim, R. Govindan, and W. Hong. "Multi-dimensional Range Queries in Sensor Networks". In Proceedings of the 1st ACM Conference on Embedded Networked Sensor Systems (SenSys), pages 63-75, 2003.

[47] B. Liu and D. Towsley. "A study on the coverage of large-scale sensor networks". In Proceedings of the 1st IEEE International Conference on Mobile Ad-hoc and Sensor Systems (MASS), pages 475-483, 2004.

[48] D. Liu, I. Stojmenovic, and X. Jia. "A scalable quorum based location service in ad hoc and sensor networks". In Proceedings of the 3rd IEEE International Conference on Mobile Ad-hoc and Sensor Systems (MASS), pages 489-492, 2006. 
[49] H. Liu, A. Nayak, and I. Stojmenovic. "Localized Mobility Control Routing in Robotic Sensor Wireless Networks". In Proceedings of the 3rd International Conference on Mobile Ad-hoc and Sensor Networks (MSN) (LNCS 4864), pages 19-31, 2007.

[50] T. Locher, P. von Rickenbach, and R. Wattenhofer. "Sensor Networks Continue to Puzzle: Selected Open Problems". In Proceedings of the 9th International Conference on Distributed Computing and Networking (ICDCN), 2008. To appear.

[51] M. Lopez-Sanchez, F. Esteva, R. L. de Mantaras, C. Sierra, and J. Amat. "Map generation by cooperative low-cost robots in structured unknown environments". Autonomous Robots, 5(1):53-61, 1998.

[52] M. Ma and Y. Yang. "Adaptive Triangular Deployment Algorithm for Unattended Mobile Sensor Networks". IEEE Transactions on Computers, 56(7):946-958, 2007.

[53] F. Martincic and L. Schwiebert. "Introduction to Wireless Sensor Networking". In I. Stojmenovic, editor, Handbook of Sensor Networks, chapter 1, pages 1-40. Wiley, 2005 .

[54] M. McMickell, B. Goodwine, and L. Montestruque. "MICAbot: A Robotic Platform for Large-Scale Distributed Robotics". In Proceedings of IEEE International Conference on Robotics and Automation (ICRA), pages 1600-1605, 2003.

[55] S. Meguerdichian, F. Koushanfar, M. Potkonjak, and M. B. Srivastava. "Coverage Problems in Wireless Ad-hoc Sensor Networks". In Proceedings of the 20th Annual Joint Conference of the IEEE Computer and Communications Societies (INFOCOM), pages $1380-1387,2001$.

[56] Y. Mei, C. Xian, S. Das, Y. C. Hu, and Y. Lu. "Replacing Failed Sensor Nodes by Mobile Robots". In Proceedings of IEEE Workshop on Wireless Sensor and Ad hoc Networks (WSAN), pages 87-92, 2006. 
[57] T. Melodia, D. Pompili, V. C. Gungor, and I. F. Akyildiz. "A Distributed Coordination Framework for Wireless Sensor and Actor Networks". In Proceedings of the 6th ACM International Symposium on Mobile Ad Hoc Networking and Computing (MobiHoc), pages 99-110, 2005.

[58] A. N. Mian, R. Beraldi, and R. Baldoni. "Survey of Service Discovery Protocols in Mobile Ad Hoc Networks". Technical Report 4/06, Universit degli Studi di Roma La Sapienza, Rome, Italy, April 2006.

[59] H. Mousavi, A. Nayyeri, N. Yazdani, and C. Lucas. "Energy Conserving MovementAssisted Deployment of Ad hoc Sensor Networks". IEEE Communications Letters, 10(4):269-271, 2006.

[60] J. O'Rourke. "Art Gallery Theorems and Algorithms". Oxford University Press, New York, U.S., 1987.

[61] M. R. Pac, A. M. Erkmen, and I. Erkmen. "Scalable Self-Deployment of Mobile Sensor Networks: A Fluid Dynamics Approach". In Proceedings of IEEE/RSJ International Conference on Intelligent Robots and Systems (IROS), pages 1446-1451, 2006.

[62] S. Poduri, S. Pattern, B. Krishnamachari, and G. Sukhatme. "Using local geometry and position control for tunable sensor network configuration". Submitted for publication, 2007. http://cres.usc.edu/pubdb_html/files_upload/547.pdf.

[63] M. H. Rahimi, H. Shah, G. S. Sukhatme, J. Heidemann, and D. Estrin. "Energy harvesting in mobile sensor networks". In Proceedings of IEEE International Conference on Robotics and Automation (ICRA), 2003.

[64] R. Ramadan, H. El-Rewini, and K. Abdelghany. "Optimal and Approximate Approaches for Deployment of Heterogeneous Sensing Devices". EURASIP Journal on Wireless Communications and Networking, 2007(1), 2007. 
[65] S. Ratnasamy, B. Karp, L. Yin, and F. Yu. "GHT: A Geographic Hash Table for DataCentric Storage". In Proceedings of the 1st ACM International Workshop on Wireless Sensor Networks and Applications (WSNA), pages 78-87, 2002.

[66] P. Santi. "Topology control in wireless ad hoc and sensor networks". ACM Computing Survey, 37(2):164-194, 2005.

[67] N. Santoro. Design and Analysis of Distributed Algorithms. Wiley, 2007.

[68] R. Sarkar, X. Zhu, and J. Gao. "Double Rulings for Information Brokerage in Sensor Networks". In Proceedings of the 12th Annual ACM International Conference on Mobile Computing and Networking (MobiCom), pages 286-297, 2006.

[69] F. E. Scheider, D. Wildermuth, and H.-L. Wolf. "Motion coordination in formations of multiple mobile robots using a potential field approach". In L. E. Parker, G. W. Bekey, and J. Barhen, editors, Distributed Autonomous Robotics Systems, volume 4, pages 305314. Springer, 2000.

[70] K.-P. Shih, H.-C. Chen, J.-K. Tsai, and C.-C. Li. "PALM: A Partition Avoidance Lazy Movement Protocol for Mobile Sensor Networks". In Proceedings of IEEE Wireless Communications and Networking Conference (WCNC), pages 2486-2491, 2007.

[71] F. Sivrikaya and B. Yener. "Time Synchronization in Sensor Networks: A Survey". IEEE Network Magazine, 18(4):45-50, 2004.

[72] I. Stojmenovic. "A scalable quorum based location update scheme for routing in ad hoc wireless networks". Technical Report TR-99-09, SITE, University of Ottawa, Ottawa, Canada, 1999.

[73] I. Stojmenovic. "Localized network layer protocols in wireless sensor networks based on optimizing cost over progress ratio". IEEE Network Magazine, 2(1):21-27, 2006. 
[74] I. Stojmenovic, D. Liu, and X. Jia. "A scalable quorum based location service in ad hoc and sensor networks". International Journal of Communication Networks and Distributed Systems, 2007. To appear. http://www.site.uottawa.ca/ ivan/ quorum-IJCNDS-F-prev.pdf.

[75] I. Stojmenovic and S. Olariu. "Data-Centric Protocols for Wireless Sensor Networks". In I. Stojmenovic, editor, Handbook of Sensor Networks, chapter 13, pages 417-456. Wiley, 2005.

[76] K. Sugihara and I. Suzuki. "distributed algorithms for formation of geometric patterns with many mobile robots". Journal of Robotic Systems, 13(3):127-139, 1996.

[77] J. B. Tchakarov and N. H. Vaidya. "Efficient Content Location in Wireless Ad Hoc Networks". In Proceedings of the 5th IEEE International Conference on Mobile Data Management (MDM), pages 74-85, 2004.

[78] G. Wang, G. Cao, and T. L. Porta. "Movement-Assisted Sensor Deployment". In Proceedings of the 23rd Annual Joint Conference of the IEEE Computer and Communications Societies (INFOCOM), volume 4, pages 2469-2479, 2004.

[79] G. Wang, G. Cao, and T. L. Porta. "Proxy-Based Sensor Deployment for Mobile Sensor Networks". In Proceedings of the 1st IEEE International Conference on Mobile Ad-hoc and Sensor Systems (MASS), pages 493-502, 2004.

[80] G. Wang, G. Cao, T. L. Porta, and W. Zhang. "Sensor Relocation in Mobile Sensor Networks". In Proceedings of the 24rd Annual Joint Conference of the IEEE Computer and Communications Societies (INFOCOM), pages 2302-2312, 2005.

[81] K. Wu, Y. Gao, F. Li, and Y. Xiao. "Lightweight Deployment-Aware Scheduling for Wireless Sensor Networks". Mobile Networks E Applications Journal, 10(6):837-852, 2005 . 
[82] B. Yamaguchi and T. Arai. "distributed and autonomous control method for generating shape of multiple mobile robot group". In Proceedings of IEEE/RSJ International Conference on Intelligent Robots and Systems (IROS), pages 800-807, 1994.

[83] B. Yamaguchi, A. Shultz, and W. Adams. "Mobile robot exploration and map-building with continuous localization". In Proceedings of IEEE/RSJ International Conference on Robotics and Automation (ICRA), pages 3715-3720, 1998.

[84] S. Yang, M. Li, and J. Wu. "Scan-Based Movement-Assisted Sensor Deployment Methods in Wireless Sensor Networks". IEEE Transactions on Parallel and Distributed Systems, 18(8):1108-1121, 2007.

[85] Y. Yang and M. Cardei. "Movement-Assisted Sensor Redeployment Scheme for Network Lifetime Increase". In Proceedings of the 10th ACM International Symposium on Modeling, Analysis and Simulation of Wireless and Mobile Systems (MSWiM), pages 13-20, 2007.

[86] A. Zelinksy. "A mobile robot exploration algorithm". IEEE Transactions on Robotics and Automation, 8(2):707-717, 1992.

[87] H. Zhang and J. C. Hou. "Maintaining Sensing Coverage and Connectivity in Large Sensor Networks". Ad Hoc \& Sensor Wireless Networks, 1(1-2):89-124, 2005.

[88] Y. Zou and K. Chakrabarty. "Sensor deployment and target localization in distributed sensor networks". ACM Transactions on Embedded Computing Systems, 3(1):61-91, 2004. 